\title{
Three-Dimensional Acoustic Propagation Through Shallow Water Internal, Surface Gravity and Bottom Sediment Waves
}

\author{
by \\ Alexey Alexandrovich Shmelev \\ Degree of Higher Education, Moscow State University, 2006 \\ Submitted in partial fulfillment of the requirements for the degree of \\ DOCTOR OF PHILOSOPHY \\ at the \\ MASSACHUSETTS INSTITUTE OF TECHNOLOGY \\ and the \\ WOODS HOLE OCEANOGRAPHIC INSTITUTION
}

September 2011

(c) Alexey Alexandrovich Shmelev 2011. All rights reserved.

The author hereby grants to MIT and WHOI permission to reproduce and to distribute publicly paper and electronic copies of this thesis document in whole or in part in any medium now known or hereafter created.

Author

Joint Program in Applied Ocean Science and Engineering Massachusetts Institute of Technology and Woods Hole Oceanographic Institution

August 19, 2011

Certified by

James F. Lynch

Senior Scientist, Woods Hole Oceanographic Institution

Thesis Supervisor

Accepted by

James C. Preisig

Chair, Joint Committee for Applied Ocean Science and Engineering

Woods Hole Oceanographic Institution

Accepted by

David E. Hardt

Graduate Officer, MIT Mechanical Engineering Department 


\title{
Three-Dimensional Acoustic Propagation Through Shallow Water Internal, Surface Gravity and Bottom Sediment \\ Waves
}

By

\author{
Alexey Alexandrovich Shmelev \\ Submitted to the Joint Program in Applied Ocean Science and Engineering \\ Massachusetts Institute of Technology \\ and Woods Hole Oceanographic Institution \\ on August 19, 2011, in partial fulfillment of the \\ requirements for the degree of \\ DOCTOR OF PHILOSOPHY
}

\begin{abstract}
This thesis describes the physics of fully three-dimensional low frequency acoustic interaction with internal waves, bottom sediment waves and surface swell waves that are often observed in shallow waters and on continental slopes. A simple idealized model of the ocean waveguide is used to analytically study the properties of acoustic normal modes and their perturbations due to waves of each type. The combined approach of a semi-quantitative study based on the geometrical acoustics approximation and on fully three-dimensional coupled mode numerical modeling is used to examine the azimuthal dependence of sound wave horizontal reflection from, transmission through and ducting between straight parallel waves of each type. The impact of the natural crossings of nonlinear internal waves on horizontally ducted sound energy is studied theoretically and modeled numerically using a three-dimensional parabolic equation acoustic propagation code. A realistic sea surface elevation is synthesized from the directional spectrum of long swells and used for three-dimensional numerical modeling of acoustic propagation. As a result, considerable normal mode amplitude scintillations were observed and shown to be strongly dependent on horizontal azimuth, range and mode number. Full field numerical modeling of low frequency sound propagation through large sand waves located on a sloped bottom was performed using the high resolution bathymetry of the mouth of San Francisco Bay. Very strong acoustic ducting is shown to steer acoustic energy beams along the sand wave's curved crests.
\end{abstract}

Thesis Supervisor: James F. Lynch

Title: Senior Scientist, Woods Hole Oceanographic Institution 


\section{Acknowledgments}

First of all I would like to thank my thesis advisor, Dr. James F. Lynch, for his continuous guidance, always optimistic mood and sense of humor. He also gave me a unique opportunity in taking part in the large scale Shallow Water 2006 Experiment, that put me directly into the front line of ocean sciences. His physical mind and ability to solve complicated scientific problems with simple approaches helped me develop the very important skill of physical thinking. Working with Jim throughout these five years was an enjoyable experience in my life, and I truly wish that everybody has such advisor whose positive energy never ends. I am also very pleased to have Dr. William M. Carey, Dr. Henrik Schmidt, Dr. Glen G. Gawarkiewicz and Dr. Pierre Lermusiaux on my doctoral committee. I thank them for their contributions to my thesis and for steering me along the science road. Many thanks for Arthur E. Newhall for constant and often instant help and technical assistance, as well as for opening the wonderful world of Linux operating systems to me. For the latter, I also thank my high school classmate and very good friend, Sergey Poltorak, for his $24 / 7$ cross-Atlantic advisories. I want to say a special thank you to Ying-Tsong Lin. Starting from my summer studentship at WHOI in 2005, he was unofficially my second advisor, and I think that large part of this work would not be properly done without his enormous help. His and Henrik's tutorials on numerical modeling made me proficient in a wide class of wave propagation problems, which should play a huge role in my future employment at WesternGeco. Also, I am very thankful to Dr. Siegmann from Rensselaer Polytechnic Institute and his student Kara McMahon. Regular collaboration with them significantly contributed to this work. Many thanks to the Ocean Acoustics and Signals Lab as well as to the Joint Program for the great working atmosphere. I specially thank the Office of Naval Research for the financial support of this work.

I am saying a huge and special thank you to my wife, Ekaterina Shmeleva. Her love and care to me made a little piece of Russia in the center of Cambridge. Thank 
you, Katya. I love you! My parents, Elena and Alexander, showed me this wonderful place, and also helped us financially. Thank you for this. Of course, many thanks to my "brother mafia", Sergey, Andrey and Alexander, who always kept me tuned, as well as to my whole family.

Finally, I want to say thank you to my grandfather, professor Leonid Sergeevich Kornienko, the biggest pride of our family. He passed away too early, but made a lot of impact on the science world and built a big and strong family together with my grandmother Emilia. He inspired my love of science, and I wish he saw my progress. I dedicate this thesis to the memory of him. 


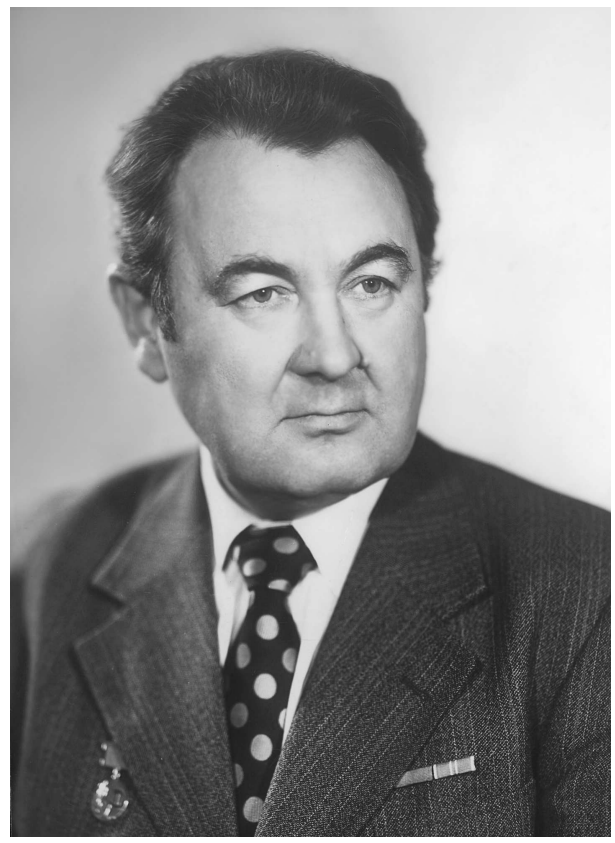

...to the memory of my grandfather Prof. Kornienko Leonid Sergeevich 


\section{Contents}

$\begin{array}{lll}1 & \text { Introduction } & 27\end{array}$

1.1 Background . . . . . . . . . . . . . . . . . . 27

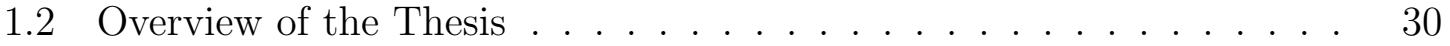

2 Observations from the Shallow Water 2006 Experiment 33

2.1 Experiment Overview . . . . . . . . . . . . . . . . . . . 33

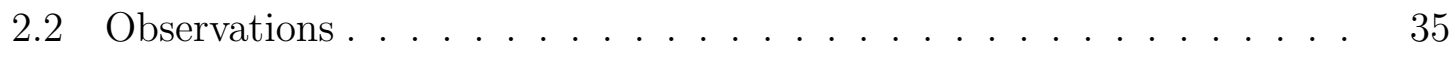

3 Brief Overview of Normal Mode Theory 41

3.1 A Point Source in a Range Independent Waveguide . . . . . . . . 42

3.2 Normal Modes for an Ideal Waveguide . . . . . . . . . . . . . . . . 44

3.3 Normal Modes for Pekeris Waveguide . . . . . . . . . . . . . 46

3.4 Normal Modes for Range Dependent Environments . . . . . . . . . . 49

4 Acoustic Normal Mode Perturbations due to Ocean Internal Waves, Bottom Sediment Waves and Surface Waves. 53

4.1 Waveguide Model . . . . . . . . . . . . . . . . . . . . . . 54

4.2 Internal Waves $\ldots \ldots \ldots \ldots \ldots$

4.3 Bottom Waves . . . . . . . . . . . . . . . . . . 66

4.4 Surface Waves . . . . . . . . . . . . . . . . . . . . . 70

5 Acoustic Propagation in Presence of Infinitely Long Parallel Waves 77

5.1 Horizontal Modal Critical Angle . . . . . . . . . . . . . . 77

$5.2 \quad$ Sharp Interface Approximation $\ldots \ldots \ldots \ldots$ 
5.3 Coupled Reflection and Transmission of Normal Modes at a Sharp Interface . . . . . . . . . . . . . . . . . . . 85

5.3.1 Plane wave mode coupling . . . . . . . . . . . . . 85

5.3.2 Point Source Solution . . . . . . . . . . . . . . . . 89

5.3.3 Horizontal Lloyd's Mirror . . . . . . . . . . . . . . . . . 92

5.4 Mode Coupled Reflection and Transmission

Through SIA Wave . . . . . . . . . . . . . . . 97

5.5 Horizontal Acoustic Ducting . . . . . . . . . . . . . . . . 101

5.5.1 Secondary Ducting ................. 103

5.6 Numerical Modeling . . . . . . . . . . . . . . . 105

6 Crossing Internal Waves 119

6.1 Physics of Crossing Acoustic Ducts . . . . . . . . . . . . . . 121

6.2 Numerical Modeling . . . . . . . . . . . . . . . . . 123

7 Applications to a More Realistic Ocean 135

7.1 Internal Waves . . . . . . . . . . . . . . . . . . 136

7.2 Surface Waves . . . . . . . . . . . . . . . . . . . . 140

7.2.1 Directional Spectrum . . . . . . . . . . . . . . . . 140

7.2.2 Numerical Modeling . . . . . . . . . . . . . . . . . 142

7.2.3 Mode coupling . . . . . . . . . . . . . . . . . 146

7.2.4 Practical Considerations . . . . . . . . . . . . . 148

7.3 Bottom Waves. . . . . . . . . . . . . . . 149

8 Conclusions $\quad 155$

8.1 Summary . . . . . . . . . . . . . . . . . . . 155

8.2 Thesis Contributions . . . . . . . . . . . . . . . . . . 158

8.3 Future Directions . . . . . . . . . . . . . . . . . . . 159

8.3.1 Analysis and Modeling . . . . . . . . . . . . . 159

8.3.2 Experimentation .................. 160 
A.1 System Configuration . . . . . . . . . . . . . . . . . 161

A.2 VLA Navigation . . . . . . . . . . . . . . . . . . . 163

A.3 HLA Navigation . . . . . . . . . . . . . . . . . . . 169

Appendix B Three-Dimensional Mode Coupling in Environments Changing in Two Dimensions

Appendix C Comparison of the Wide Angle Parabolic Equation Program with the Exact Solution 


\section{List of Figures}

2-1 (a) Location of the SW06 experiment. (b) T-geometry of the environmental and acoustic moorings during the deployment. . . . . . . . . . 34

2-2 Configuration of the horizontal and vertical line arrays connected to the hardware recording box (called Shark because of its shape). . . . 35

2-3 Position of the Naval Research Lab source, NRL300, relative to the Shark (WHOI array). . . . . . . . . . . . . 36

2-4 Demodulated replica of the NRL300 LFM signal (black line) and its envelope (red line). . . . . . . . . . . . . . . 36

2-5 Signals received along the HLA and cross-correlated with its replica (compressed pulses) are shown by black lines. Hann windowed outputs are shown by red lines. . . . . . . . . . . . . . . . . . . 38

2-6 Beamforming output for three selected receptions for transmissions during 11:30-11:37 and 12:00-12:07 on August 19, 2006. . . . . . . . . 39

3-1 A homogeneous fluid layer with pressure release surface and hard bottom (Ideal waveguide). . . . . . . . . . . . . . . .

3-2 Homogeneous fluid layer with a pressure release surface and a homogeneous, higher sound speed, bottom half space (Pekeris waveguide); vertical grazing angle regions are for the discrete and continous modal spectrum. . . . . . . . . . . . . . . .

4-1 Background water column model: two homogeneous fluid layers (upper layer having slightly higher sound speed) bounded by a free surface and a homogeneous half space bottom of higher sound speed. . . . . . . . 
4-2 Vertical modal wavenumber for the lower layer (left), and horizontal modal wavenumber (right) computed with the KRAKEN normal mode code for the waveguide model having sound speed in upper and lower layers $c_{m l}=1530 \mathrm{~m} / \mathrm{s}$ and $c_{0}=1500 \mathrm{~m} / \mathrm{s}$ respectively, density $\rho_{0}=$ $1000 \mathrm{~kg} / \mathrm{m}^{3}$, depth of the interface between water layers $D=15 \mathrm{~m}$, and bottom of depth $H_{b o t}=80 \mathrm{~m}$ with sound speed $c_{b o t}=1800 \mathrm{~m} / \mathrm{s}$ and density $\rho_{b o t}=2000 \mathrm{~kg} / \mathrm{m}^{3}$. . . . . . . . . . . . . .

4-3 First four normal modes for the background water column at $100 \mathrm{~Hz}$ (left), $200 \mathrm{~Hz}$ (middle), and $400 \mathrm{~Hz}$ (right). . . . . . . . . . . 58

4-4 Modal normalization constant $A_{m}$ for the background waveguide at frequencies $100 \mathrm{~Hz}, 200 \mathrm{~Hz}$ and $400 \mathrm{~Hz}$. . . . . . . . . . . 58

4-5 Depression internal waves represented by lowering the interface between water layers. . . . . . . . . . . . . . . . 59

4-6 Correction to the background horizontal modal wavenumber for frequency $200 \mathrm{~Hz}$ due to the internal wave of fifteen meter amplitude, computed numerically (solid line), using perturbation method (dashed), and using a three step iterative perturbation method (dot-dashed). .

4-7 Normal modes 3-5 for background waveguide model (left) and inside a wave of fifteen meter amplitude (right). Dashed line represents the water-bottom interface; the interface between water layers (thermocline) is shown as a dotted line . . . . . . . . . . . . . .

4-8 Two methods of the background water column multi-step perturbation due to internal waves: stepwise displacement of the thermocline (left); stepwise increase of sound speed (right). . . . . . . . . . . . .

4-9 Correction to the background horizontal modal wavenumber for frequencies $100 \mathrm{~Hz}, 200 \mathrm{~Hz}$ and $400 \mathrm{~Hz}$, due to internal wave of fifteen meter amplitude. . . . . . . . . . . . . . . . .

4-10 Bottom waves approximation by lifting the water-seabed interface. . . 
4-11 Correction to the background horizontal modal wavenumber for frequency $200 \mathrm{~Hz}$ due to presence of bottom wave of one meter amplitude, computed numerically (solid line), using linear approximation (4.41) (dot-dashed line), and using simplified linear approximation (4.44) (dashed line). . . . . . . . . . . . . . . .

4-12 Correction to the background horizontal modal wavenumber for frequencies $100 \mathrm{~Hz}, 200 \mathrm{~Hz}$ and $400 \mathrm{~Hz}$, due to bottom wave of one meter amplitude. . . . . . . . . . . . . . . . .

4-13 Ocean surface waves approximated by elevation and depression of the

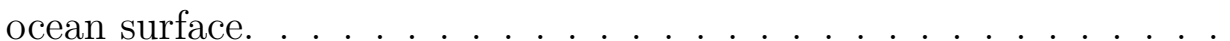

4-14 Schematic view of two steps water column perturbation due to surface wave crest (trough): thermocline depression (elevation) by $\Delta H_{\text {sur }} / 2$ followed by the water depth increase (decrease) by $\Delta H_{\text {sur }} / 2 \ldots$. . . . 72

4-15 Modal wavenumber variation amplitude for frequency $200 \mathrm{~Hz}$ due to the presence of surface waves with one meter amplitude. The numerical solution is plotted with a solid line; the dashed line represents the first order approximation. . . . . . . . . . . . . . . . 75

4-16 Numerical solution for the modal wavenumber variation amplitude at frequencies $100 \mathrm{~Hz}, 200 \mathrm{~Hz}$ and $400 \mathrm{~Hz}$, due to presence of surface waves with one meter amplitude. . . . . . . . . . . . 
5-1 Top view of the infinitely long waves (for example, internal waves) with crests or troughs parallel to the $x$-axis. The horizontal wavenumber of mode $n$ is schematically shown in gray shading. The white unshaded region corresponds to the background unperturbed waveguide with wavenumber $k_{r n}^{0}$; the wave crest is characterized by the perturbed value $k_{r n}^{1}$. The horizontal grazing angle of a modal ray is defined as the angle between the direction of the mode $n$ ray and the direction of the wave crest. Modal rays incident from the source are divided into two groups. Rays from the first group are characterized by smaller than critical horizontal grazing angles and are refracted by the first wave back into the half space of positive $y$-values. The second group of rays have steeper than critical horizontal grazing angles. These rays penetrate through the waves and propagate into the half space of negative $y$-values. . . . . . . . . . . . . . . . . .

5-2 Horizontal critical angles associated with internal waves of fifteen meter amplitude (upper panel), bottom wave of one meter amplitude (middle panel) and surface wave of one meter amplitude (lower panel). Parameters of the waveguide are: $c_{0}=1500 \mathrm{~m} / \mathrm{s}, c_{m l}=1530 \mathrm{~m} / \mathrm{s}, c_{b o t}=1800$ $\mathrm{m} / \mathrm{s}, \rho_{0}=1000 \mathrm{~kg} / \mathrm{m}^{3}, \rho_{b o t}=2000 \mathrm{~kg} / \mathrm{m}^{3}, H_{b o t}=80 \mathrm{~m}, D=15 \mathrm{~m}$. Wave amplitudes are: $\Delta H_{i w}=15 \mathrm{~m}, \Delta H_{b o t}=1 \mathrm{~m}$ and $\Delta H_{\text {sur }}=1 \mathrm{~m}$. Blue dashed line shows the observed $4 \mathrm{deg}$ fluctuation of the angle of arrival due to presence of internal waves (see Chapter 2). . . . . . .

5-3 Schematic diagram showing traditional division of acoustic propagation into four sectors of grazing angles, having different propagation regimes: strong mode coupling $(\mathrm{MC})$, nearly adiabatic propagation (AD), horizontal refraction (HR), and horizontal focusing (HF). . . .

5-4 Idealized wave constructed by the two sharp vertical interfaces sepa-

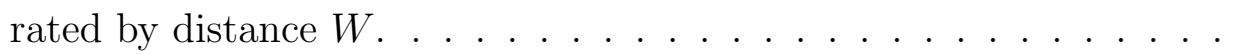


5-5 Schematic diagram showing sharp interface approximation (SIA) of internal waves, bottom waves and surface waves in the two-layer waveguide model. . . . . . . . . . . . . . . . . . . . . .

5-6 Plane wave mode coupling at the sharp interface. Incident pressure field consists of the single mode $n$ of unit amplitude with grazing angle $\chi_{n}$. Along the interface, it couples into all propagating modes $1 . . M$ of both reflected and transmitted fields. . . . . . . . . . . . . .

5-7 Absolute values of the plane wave coupled reflection coefficient $R_{n m}$ (top panel) and transmission coefficient $T_{n m}$ (bottom panel) for incident mode three $(n=3)$ and frequency $100 \mathrm{~Hz}$ for a sharp interface between background waveguide model $\left(c_{0}=1500 \mathrm{~m} / \mathrm{s}, c_{m l}=1530\right.$ $\mathrm{m} / \mathrm{s}, c_{b o t}=1800 \mathrm{~m} / \mathrm{s}, \rho_{0}=1000 \mathrm{~kg} / \mathrm{m}^{3}, \rho_{b o t}=2000 \mathrm{~kg} / \mathrm{m}^{3}, H_{b o t}=80$ $\mathrm{m}$ and $D=15 \mathrm{~m}$ ) and a perturbation due to a twenty five meter amplitude internal wave. . . . . . . . . . . . . . . . .

5-8 Same as in Fig. 5-7, but for mode one incident. . . . . . . . . . . . . 88

5-9 Geometrical approximation of the coupled mode horizontal reflection from and transmission through the sharp interface. . . . . . . . .

5-10 Modal amplitudes for the case of mode three incident from a point source located at $\left(x_{s}, y_{s}\right)=(0,500) \mathrm{m}$. There is a twenty five meter amplitude infinitely wide internal wave with its sharp interface along

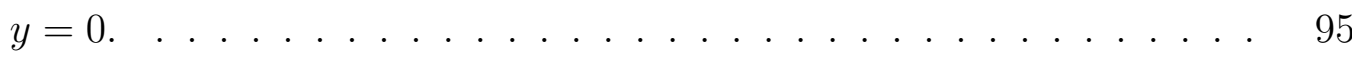

5-11 Same as in Fig. 5-10, but for the mode one incident. . . . . . . . . . 96

5-12 Mode coupling diagram for normal incidence of mode $n$ upon a SIA wave. Mode $n$ partially couples into mode $m$ at the front interface. At a certain resonant width of the wave, mode $m$ uncouples back into mode $n$ at the back interface. . . . . . . . . . . . . 
5-13 Mode coupling diagram for mode $n$ incident upon a SIA wave a with steep (other than normal) grazing angle. Weak refraction of the coupled modes at these angles makes both broken-line rays coincide with each other and with a straight (dashed) line. If one measures the modal intensities behind the wave along the arc of steep grazing angles (magenta line), significant fluctuations will be noticed as the effective width of the wave passes through its resonant values. . . . . . . . .

5-14 Tophat-like shape of the horizontal wavenumber in presence of the SIA wave. . . . . . . . . . . . . . . . . 100

5-15 Upper panel: acoustic modal ducting for the source placed between two idealized waves. Bottom panel: acoustic modal antiducting for a source located inside an internal wave: modal rays nearly parallel to the wave crest are refracted out from the wave with the critical grazing

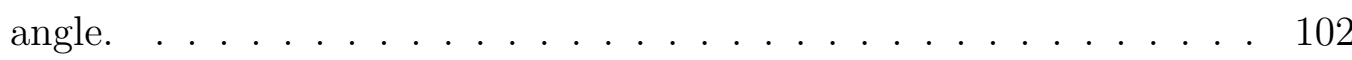

5-16 Secondary ducting due to mode tunneling. Partially ducted energy leaks out from the duct at each interaction with the waves. . . . . . . 104

5-17 Secondary ducting due to mode coupling. Mode $n$ couples ot front or back or both interfaces of the wave into mode $m$ with higher critical angle. At certain grazing angles $\chi_{n}$, transmitted coupled energy of mode $m$ is ducted. . . . . . . . . . . . . . . . . . 105

5-18 Upper panel: internal wave of depression with amplitude of $25 \mathrm{~m}$ and hyperbolic secant squared shape (black line). Half amplitude wave width is $130 \mathrm{~m}$. The blue line represents hundred steps dividing the wave into locally range independent sectors used in numerical modeling. Middle panel: changes in the horizontal modal wavenumbers across the soliton internal wave with respect to background water column values. Bottom panel: Normal mode horizontal critical angles. 108 
5-19 Amplitudes of modes 1-4 for the case of incident mode one with unit amplitude at one meter distance from the source and single internal wave of twenty five meter amplitude centered at $y_{\text {wave }}=0 \mathrm{~m}$. Source is located at $y_{s}=500 \mathrm{~m}$ (red circle) . . . . . . . . . . . .

5-20 Same as in Fig. 5-19, but for incident mode three. . . . . . . . . . . .

5-21 Upper panel: a bottom sediment wave of two meter amplitude with hyperbolic secant squared shape (black line). Half height width is $130 \mathrm{~m}$. The blue line represents hundred steps dividing the wave into locally range independent sectors to be used in numerical modeling. Middle panel: changes in the horizontal modal wavenumbers across the bottom sediment wave with respect to background water column values. Bottom panel: normal mode horizontal critical angles. . . . . 113

5-22 Amplitudes of modes 3-6 for incident mode five with unit amplitude at one meter distance from the source and a single bottom sediment wave of two meter amplitude centered at $y_{\text {wave }}=0 \mathrm{~m}$. Source is located at $y_{s}=500 \mathrm{~m}($ red circle $) \ldots \ldots \ldots \ldots$

5-23 Horizontal ducting of normal mode. Acoustic source located at $y_{s}=$ $0 \mathrm{~m}$ is in between two internal waves centered at $y_{\text {wave }}= \pm 300 \mathrm{~m}$. Incident field consists of a single mode one with unit amplitude at one meter distance from the source. . . . . . . . . . . . . . .

5-24 Secondary ducting. An acoustic source at $\left(x_{s}, y_{s}\right)=(0,500) \mathrm{m}$ is located just outside of the duct composed of two internal waves centered at $y_{\text {wave } 1}=0 \mathrm{~m}$ and $y_{\text {wave } 2}=-600 \mathrm{~m}$. The incident field consists of a single mode one of unit amplitude at one meter distance from the source.117

5-25 Same as in Fig. 5-24, but for incident mode two. . . . . . . . . . . . . 118

6-1 SAR image of the internal waves in the SW06 experimental area showing multiple crossings of the curved wave trains. Taken on July 23,

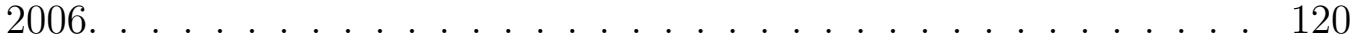


6-2 Schematic view of ducted acoustic energy interaction with crossing waves. At steep angles of wave crossing (upper panel), mode coupling is considerable, and ducted energy is scattered by interactions with multiple waves. At shallow angles of wave crossing (bottom panel), part of the modal energy is pulled out of the duct by reflecting from the first crossing wave (red solid ray), and another part tunnels (red dashed ray) or propagates (blue ray) through the first crossing wave and becomes ducted between the first and the second crossing waves (cross-ducting). . . . . . . . . . . . . . . . .

6-3 Left plot: smoothed sound speed cross-section along $x=0 \mathrm{~m}$. Red circle shows the source location (note that the depth of the source is not specified, since a single mode starting field is used in the program). Right plot: smoothed density column. . . . . . . . . . . . . . 125

6-4 Crossing of two identical pairs of internal waves at forty degrees. White dashed lines indicate the wave crests. Depth integrated intensity (re 0 $\mathrm{dB}$ at $1 \mathrm{~m}$ from the source) is shown in the top panel, with an enlarged crossing region in the second panel from the top. Intensities of modes one (initial) and two are shown in the lower two panels. . . . . . . . . 127

6-5 Same as in Fig. 6-4, but for a wave crossing angle of eleven degrees. . 128

6-6 Same as in Fig. 6-4, but for a wave crossing angle of seven degrees. . 129

6-7 Same as in Fig. 6-4, but for a wave crossing angle of four degrees. . . 130

6-8 Same as in Fig. 6-4, but for a wave crossing angle of two degrees. . . 131

6-9 Crossing of the parallel internal waves with a single internal wave at four degrees. White dashed lines indicate the wave crests. Depth integrated intensity (re $0 \mathrm{~dB}$ at $1 \mathrm{~m}$ from the source) is shown in the top panel with enlarged crossing region in the second panel from the top. Intensities of modes one (initial) and two are shown in the lower two panels. . . . . . . . . . . . . . . . . . . . . 132

6-10 Same as in Fig. 6-9, but for a wave crossing angle of two degrees. . . 133 
7-1 SAR image of internal wave activity $20 \mathrm{~km}$ shoreward of the SW06 site taken on August 8, 2006. . . . . . . . . . . . . 137

7-2 Left panel: homogeneous shelfbreak and continental shelf do not affect curvature of the internal wave's fronts. Right panel: interaction of barotropic tides with bottom canyons or shoals results in point sources of internal waves. . . . . . . . . . . . . . . . . . . . . . 138

7-3 Experimental setup with an acoustic source and several closely spaced receivers for increasing the number of closely spaced along wave acous-

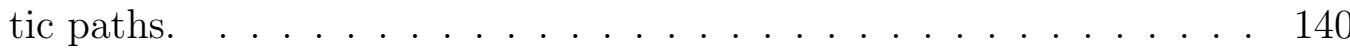

7-4 (a) Directional spectrum for surface swell with $H_{1 / 3}=2 \mathrm{~m}, T_{1 / 3}=17$ $\mathrm{s}, s_{\max }=75$ and $\phi_{0}=0$ deg. (b) Directional spectrum shown in (a) plotted for the dominant azimuth $\phi_{0}$. . . . . . . . . . . . . . . 143

7-5 (a) Realization of the sea surface elevation based on directional spectrum shown in Fig. 7-4. (b) Enlarged view of (a) shown for the region inside the dashed line. . . . . . . . . . . . . . . . . . . . . . . . . 144

7-6 Amplitudes of modes 1, 3, 5 and 7 for adiabatic propagation from the source, which is located at $\left(x_{s}, y_{s}\right)=(0,0) \mathrm{m}$, and has frequency $100 \mathrm{~Hz} .145$

7-7 (a) Vertical sharp interface dividing unperturbed waveguide and its perturbation due to surface depression of amplitude $2 \mathrm{~m}$. (b) Pressure field solution at the unperturbed side of sharp interface (black) and at the unperturbed side (blue). Red line represents the difference in solutions on both sides. . . . . . . . . . . . . . . . . . . . . 147

7-8 Plane wave mode coupled reflection (left plot) and transmission (right plot) coefficients for the normal incidence upon the sharp interface between unperturbed waveguide and its perturbation due to surface depression of amplitude $2 \mathrm{~m}$ and frequency $100 \mathrm{~Hz}$. . . . . . . . . . 148

7-9 Three-dimensional view of the bottom relief at the mouth of San Francisco Bay. Dashed line shows the domain selected for the three-dimensional acoustic propagation numerical modeling. . . . . . . . . . . . . . 152 
7-10 (a) Bathymetry of the region selected for the acoustic propagation domain (shown by dashed line in Fig. 7-9). (b) Bathymetry transect along $x=1000 \mathrm{~m} \ldots \ldots \ldots \ldots$

7-11 Depth integral of the acoustic intensity for a source of frequency 500 Hz located at $\left(x_{s}, y_{s}, z_{s}\right)=(0,0,10) \mathrm{m} . \ldots \ldots \ldots 154$

A-1 Top view of the LBL system elements and selected hydrophone channels for LBL navigation. . . . . . . . . . . . . . . . . . . . . 163

A-2 Example of the total travel time from the interrogator to the top VLA hydrophone channel $\mathrm{CHO}$ for the direct path (upper panel) and for the two-leg path through the East transponder (lower panel). . . . . . . . 165

A-3 One of the selected sound speed columns (a) and the corresponding eigenrays connecting the interrogator with the West transponder (b) and the interrogator with the East transponder $(\mathrm{c}) . \ldots 166$

A-4 One of the sound speed columns at the VLA (a) and computed acoustic "ray fan" (b). Left, central and right groups of circles correspond to the possible locations of the channel $\mathrm{CHO}$ according to the travel time integral along each ray for the paths East transponder-to-CH0, interrogator-to- $\mathrm{CHO}$ and West transponder-to-CH0 respectively. . . .

A-5 Possible locus of the channel $\mathrm{CH} 0$ in the vertical planes connecting the West transponder and $\mathrm{CHO}(\mathrm{a})$, the Interrogator and $\mathrm{CHO}(\mathrm{b})$ and the East transponder and $\mathrm{CHO}(\mathrm{c})$. Blue and black colors denote water borne and surface reflected rays respectively. Red rhombs indicates the nominal position of the channel CHO . . . . . . . . . . . . . 168

A-6 Horizontal positions of the top VLA hydrophone channel CH0. The $y$-axis points true North and the $x$-axis points true East. . . . . . . . 169 
A-7 Example of the horizontal ranges for the eigenrays connecting the East transponder and channel CH27. Green and blue dots represent along bottom and refracted water borne eigenrays, and black dots represent surface reflected eigenrays. The eellow line is the low pass filtered horizontal ranges for the dominant (blue) eigenray. Red dots show a combination of selected horizontal ranges used for the final localization. 172

A-8 Localized positions of HLA channels CH17, CH27 and CH37. . . . . . 172

A-9 X-component of HLA channels locations (CH17, CH27 and CH37). . 173

A-10 Eastern component of the ocean current for the $68 \mathrm{~m}$ depth bin at the SW30 mooring. . . . . . . . . . . . . . . . . . 173

B-1 The range dependent part of the waveguide $\left[\begin{array}{ll}y_{a} & y_{b}\end{array}\right]$ is divided by $N$ vertical interfaces $y_{j}, j=1 . . N$ into $N+1$ segments such that $y_{1}=y_{a}$, $y_{N}=y_{b}$. Within each segment, the waveguide properties don't change significantly and are thus locally range independent. The solution within each homogeneous segment $j$ is a sum of right and left going plane waves with amplitudes $\Phi_{m}^{(j)+}$ and $\Phi_{m}^{(j)-}$ respectively. Segment $j_{s}$ containing the acoustic source has an additional source term $\tilde{p}^{s}$ in the solution. . . . . . . . . . . . . . . . . . . 177

B-2 New integration contour shifted into the complex plane away from the poles. . . . . . . . . . . . . . . . . . . 180

C-1 Output of the three-dimensional wide angle parabolic equation program for the same scenario as shown in Fig. 5-24. . . . . . . . . . . . 184 


\section{List of Tables}

A.1 Deployed and refined positions of the LBL elements. . . . . . . . . 162

A.2 Nominal positions of the selected hydrophone channels. . . . . . . 162

A.3 Average values and standard deviations for the coordinates of the HLA

hydrophone channels CH17, CH27 and CH37. . . . . . . . . 171 


\section{Chapter 1}

\section{Introduction}

\subsection{Background}

Low frequency acoustic propagation in shallow waters has received a lot of attention in the last several decades. While naval operations and underwater surveillance were some of the primary motivations of studying this field, a number of scientific and commercial applications such as studying the dynamics of the ocean, underwater long range communications or tracking marine mammals showed a growing interest in shallow water acoustics. Early studies were focused on sound interactions with the sea bottom whose geological structure, slope and roughness are known to be important parameters for acoustic energy loss [1]. Along with the seabed, there are many other oceanographic features that create loss or gain of acoustic energy and are as important. Propagation of sound waves through meso-scale eddies or a meandering shelfbreak front can result in a significant variability of acoustic pulse travel time and the acoustic depth energy distribution. On the ocean fine scale, nonlinear internal waves [2], that are common for most of the continental shelves in the summer time or at low latitudes, are known to be very important features for low frequency acoustic propagation and scattering. In the 1980's Yellow Sea

experiments, Zhou et al. [3] showed and theoretically explained very strong (up to $25 \mathrm{~dB}$ ) resonance-like intensity fluctuations that are both frequency and internal wave direction dependent. Preisig and Duda [4] studied the impact of individual 
soliton-type internal waves on normal mode coupling physics and clearly showed its dependence on wave width, amplitude and acoustic frequency. Colosi and Flatte theoretically showed [5] that acoustic normal mode coupling induced by deep water internal waves largely contributes to scattering of low frequency signals at long ranges. In the 1995 Shallow Water Acoustics in a Random Medium experiment (SWARM95) [6], strong mode coupling was observed and studied statistically [7] for the across internal wave propagation direction. Also as part of the SWARM95 experiment, Badiey [8] measured acoustic signals for an along waves path and showed that strong intensity fluctuations are caused by horizontal ducting of the acoustic signal between internal waves. This was the first experimental evidence of an out of vertical plane acoustic interaction with internal waves, which was predicted theoretically in [9] and numerically in $[10,11]$. The Shallow Water 2006 Experiment (SW06) [12, 13] was the first effort that concentrated on measuring the fully three-dimensional variability of the water column as well as acoustic signals coming from both the across and along internal waves directions. For that experiment, both intensity and angle of arrival fluctuations analyses [14], that are also presented in this thesis, were made for the along internal wave acoustic track with a fixed source and receiving array. The first experimental evidence of the horizontal Lloyd's mirror, an inherently threedimensional acoustic effect, was clearly observed in SW06 by Badiey et. al. [15]. Also in SW06, Lynch et. al. [16] presented results on intensity fluctuations and their azimuthal dependence for a mobile source acoustic signal. As a result of SW06, studies of three-dimensional low frequency acoustic, including the effects of internal waves natural curvature [17] and termination [18, 19], were extensively developed.

Most of the theoretical work on three-dimensional acoustic propagation in the along internal wave direction has been made under the adiabatic approximation, and mode coupling for these scenarios was not considered important. However, refraction of internal waves in shallow waters, as well as the existence of strong scatterers such as bottom canyons, causes internal wave front's curvature and wave crossings. These crossings are observed [20] in almost every region of continental shallow waters when nonlinear internal wave activity exists. Since the angle of crossing varies widely, from 
zero to ninety degrees, acoustic energy ducted in between internal waves from one train may interact with another train of waves at all possible angles, and the effects of energy transfer due to normal mode coupling need to be addressed.

Besides internal waves, there are two other types of shallow water inhomogeneities that we will consider to be important for three-dimensional acoustics: bottom sediment waves and surface gravity waves. Bottom waves are common in shallow water and on the shelfbreak in the forms of small (ripples), medium (megaripples) and large (sand waves) transverse bedforms [21]. Note that in the literature the term "sand dunes" can refer to both sand waves and megaripples. Bottom ripples have wavelengths of centimeters, whereas the length scales of megaripples and sand waves are tens and even hundreds of meters. The last two are of more importance for the low frequency acoustics because of their size. Middleton and Southard [22] summarized the major distinctions between megaripples and sand waves based on their formation mechanisms and the seabed type. He concludes that sand waves are more regular and have longer crest continuity than megaripples. Reeder et al. [23] has recently observed large sand waves on the continental slope of the Northern South China Sea that have crest to crest wavelengths exceeding 350 meters and amplitude exceeding 16 meters. He discovered that these waves were generated by strong interaction of nonlinear internal waves with the continental slope. In similar observations at the Japan Sea shelf, Serebryany showed very good correlation between the shapes of nonlinear internal waves and sand waves located one above another [24]. In his thesis, de Koning [25] applied a stochastic approach to describe the sand waves geometrical distribution in the North Sea and provided its directional spectrum. The literature and bathymetry data suggest that the existence of megaripples and sand waves of various amplitudes is common for the majority of continental shelf regions and shelfbreaks. Although many of the mechanisms of their formation are known and well understood now, their impact on three-dimensional acoustic propagation has not yet been studied, and is of great interest to us. For the purposes of this thesis, we will refer to both types of bedforms above as to bottom sediment waves.

Ocean surface waves with relatively short wavelengths generally act as random 
scatterers for acoustic signals [1]. If wave breaking occurs, they are also known to be strong generators of ambient noise [26]. When studying low frequency (ten to a thousand $\mathrm{Hz}$ ) acoustics, longer wavelength ocean surface waves are of particular interest. In a developing storm area, longer wavelength, and therefore longer period, surface waves are generated last. However, once generated, these long waves, also called swell, travel with faster speed and to longer distances than the shorter (sea) waves [27]. A striking example of swell that traveled along a great circle from the Indian ocean to California was digitally recorded and described in detail by Munk [28]. Similar measurements by Goda [29] showed a significant wave height of two meters for swell that traveled over nine thousand kilometers, and had a narrow directional spectrum. Similarly to internal waves and bottom waves, long wavelength narrow directional swells are believed to have a noticeable three-dimensional impact on low frequency acoustic propagation, which has not been investigated before.

\subsection{Overview of the Thesis}

This work is dedicated to studying the three-dimensional interaction of low frequency acoustic signals with ocean internal, surface and bottom waves. As a motivation for the theoretical study, in Chapter 2 we present our observations of the horizontal angle of arrival variability noticed in the SW06 data for a fixed acoustic source and receiver array. In Chapter 3, we will briefly summarize the fundamentals of acoustic normal mode theory, a primary tool used to approach our problem. Theoretical analysis of normal mode properties and their perturbation due to the presence of each of the three types of waves is studied using the example of an idealized, but insightful waveguide model, and this is presented in Chapter 4. In Chapter 5, a theoretical approach is used for studying three-dimensional effects of acoustic propagation in presence of straight and parallel waves of each type. These effects are also illustrated by fully threedimensional coupled mode numerical modeling. A natural and important property of internal waves is to have crossing structures, and this is discussed with applications to three-dimensional acoustics and also simulated numerically in Chapter 6. In Chapter 
7, we present a computational look into acoustic propagation through real ocean environments with each type of wave present, as well as discuss our suggestions for possible future experiments. The summary and conclusions of this work are found in Chapter 8 . 


\section{Chapter 2}

\section{Observations from the Shallow Water 2006 Experiment}

\section{$2.1 \quad$ Experiment Overview}

The Shallow Water 2006 (SW06) experiment $[12,13]$ was conducted off the coast of New Jersey (Fig. 2-1a), in the same area as the previous SWARM95 [6] experiment, and lasted over two months (mid-July - mid-September). The experiment involved sixty two environmental and acoustic moorings, seven research vessels, ten ocean gliders, mobile acoustic sources, airborne and satellite measurements, and the mutual effort of many institutions and universities. One of the primary goals was the detailed study of the linear and nonlinear internal waves found in that region and their three-dimensional interaction with low and medium frequency acoustic signals. To accomplish this goal, environmental moorings were deployed in a T-geometry (Fig. 2-1b) that includes a thirty kilometer path along the shelfbreak at the eighty meter isobath (stem of the T) and a fifty kilometer path across the shelfbreak (top of the T). Acoustic sources were deployed at the outer ends of the $\mathrm{T}$ and an L-shaped acoustic receiving array was placed at the intersection of the along and across shelfbreak paths (WHOI array in Fig. 2-1b). A number of closely spaced environmental moorings were concentrated near the acoustic array to measure the three-dimensional structure of the internal wave fields. This overall setup allowed the measurement of 


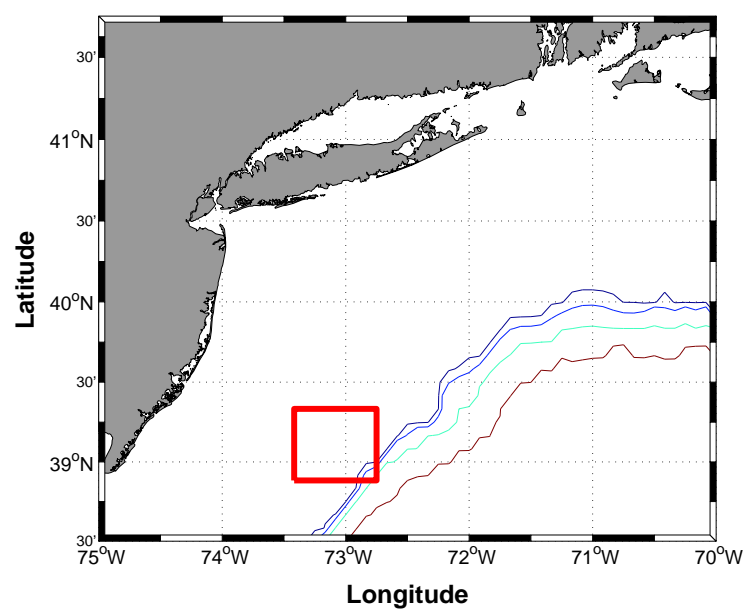

(a)

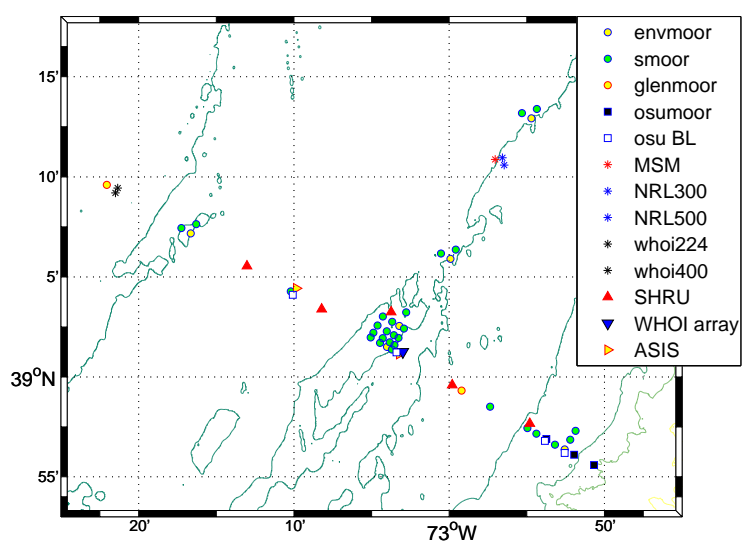

(b)

Figure 2-1: (a) Location of the SW06 experiment. (b) T-geometry of the environmental and acoustic moorings during the deployment.

acoustic signals traveling along and across shelfbreak, the latter of which also is nearly perpendicular to mean direction of the prominent nonlinear internal wave crests. The amplitudes of nonlinear internal waves observed at the moorings along eighty meter isobath varied from a few to $25 \mathrm{~m}$ with mean amplitude of approximately $8 \mathrm{~m}$ [30].

Physically the acoustic L-shaped WHOI array consisted of horizontal and vertical line arrays (HLA/VLA) connected by a heavy steel box with electronic hardware. This box, also called Shark because of its shape, was simultaneously recording the signals received by the HLA and VLA hydrophones (Fig. 2-2). The HLA had 31 evenly 


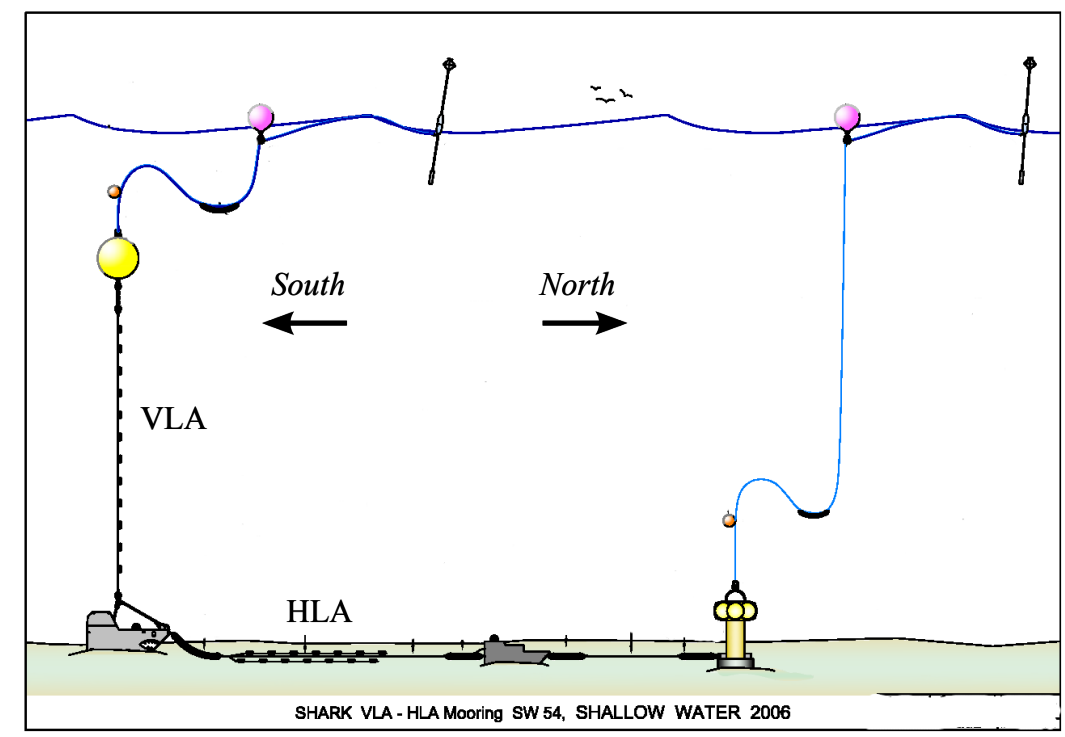

Figure 2-2: Configuration of the horizontal and vertical line arrays connected to the hardware recording box (called Shark because of its shape).

spaces hydrophones and a total aperture of 465 meters. During the deployment, the HLA was stretched tight between the Shark node at the south end and HLA tail sled node at the north end. The VLA consisted of 16 hydrophones of total aperture 66 meters and was kept tightened by large flotation sphere at 11 meters depth. A long baseline acoustic navigation system was also deployed and used both for tracking the lateral movement of the HLA and the VLA tilt caused by the semiduirnal tides and internal wave induced currents (see Appendix A for details).

\subsection{Observations}

Though SW06 was perhaps the third experiment to examine low frequency acoustic transmission along internal waves (after the Yellow Sea experiment and SWARM95), it was the first to provide extensive environmental measurements and support along such paths. Our initial interest here is to look at the signals coming from along the shelfbreak directions. The Naval Research Lab (NRL) linear frequency modulated source (denoted as NRL300 in Fig. 2-1b) was deployed at a depth of 72 meters at the outer end of the along shelf path. The straight line horizontal distance and the bearing relative to the True North to this source from the Shark were $18 \mathrm{~km}$ and 27.2 


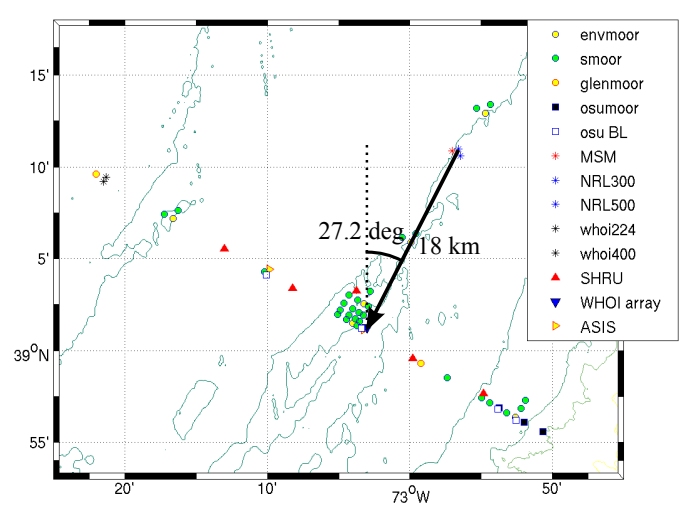

Figure 2-3: Position of the Naval Research Lab source, NRL300, relative to the Shark (WHOI array).

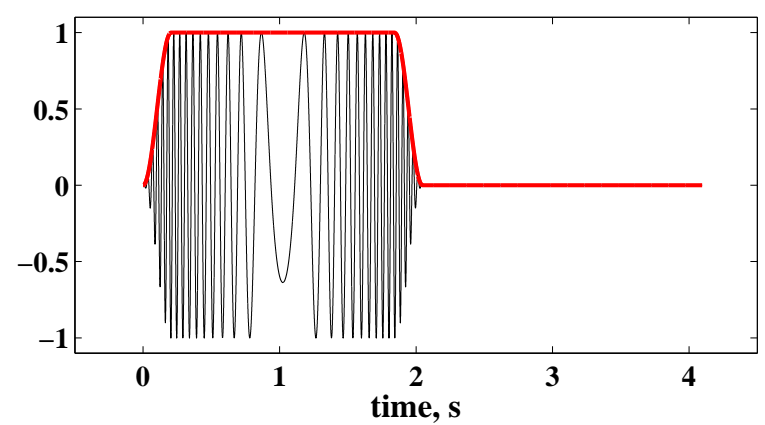

Figure 2-4: Demodulated replica of the NRL300 LFM signal (black line) and its envelope (red line).

deg respectively (Fig. 2-3). The source radiated an upsweeping LFM signal (270-330 $\mathrm{Hz}$ ) with a length of 2.048 seconds. Ten percent of the signal length (0.2048 seconds) in the beginning and at the end are tapered with a cosine function. Signal was repeated every 4.096 seconds, i.e. with 2.048 seconds of silence between repeating. A demodulated replica of the signal (i.e. with central frequency $300 \mathrm{~Hz}$ extracted) and its envelope are shown in Fig. 2-4. Transmissions were scheduled every half hour with total duration of seven minutes. For the central frequency of $300 \mathrm{~Hz}$, a reference sound speed $1500 \mathrm{~m} / \mathrm{s}$ and a horizontal range of $18 \mathrm{~km}$, the first Fresnel zone has a diameter of $752 \mathrm{~m}$ which covers the HLA aperture. This allows us to use plane wave approximation for the wave front. Figure 2-5 illustrates the example of the received at the HLA signals cross-correlated with its replica shown in black lines (these outputs are called compressed pulses). As one can see from the figure, normal mode arrivals 
are not totally time resolvable. The more detailed analysis of signal received at the VLA showed that the first peak was often a result of the interference between the first two (and highest amplitude) modes. In order to separate modes one and two from the other arrivals, the signal was multiplied by a Hann window of 0.2 seconds length and centered at the first peak of the compressed pulses. The windowed signals are shown by red lines in Fig. 2-5. Using the shape of the HLA and its hydrophone locations found in Appendix A, the angle of the signal arrival is then found by plane wave beamforming [31] of the filtered pulse compressed signals. For our signal processing, we assumed the waveguide properties along the HLA to be locally range independent for the time frame of each transmission and used the water column properties for the VLA location. This is a reasonable assumption for our application since the signal's distortion over the length scale of the HLA aperture is considered very small compared to the total traveled distance of $18 \mathrm{~km}$. However, it is not perfect, especially when strong acoustic scatterers like a internal solitons or high sea swell are over the array. We also used the group speed of mode one, that was computed with KRAKEN normal mode code [32], for beamforming computations. We note that our filtered signal is a superposition of modes one and two, and there is thus associated uncertainty in angle of arrival estimation that needs to be addressed. Using the first order assumption of range independent waveguide and adiabatic propagation of normal modes along the transmission path, this uncertainty was evaluated numerically to be 1.1 degrees for the examples we will consider below.

Scheduled transmissions started every half hour, with a duration of 7 minutes and 30 seconds. Figure 2-6 shows the output of beamforming for three selected receptions, one for the transmission session during 11:30-11:37 UTC and two others for 12:0012:07 UTC on August 19, 2006. At this time, internal waves activity with amplitudes exceeding $18 \mathrm{~m}$ was registered at the VLA. One can clearly see from the figure that over the time window of $37 \mathrm{~min}$ (two transmission sessions), the difference in the maximum of beamforming output (horizontal beam wobble) is approximately four degrees. The modal interference uncertainty is a part of the beam wobble, but it constitutes only 25 percent of this phenomenon. This beam wobble was not observed 


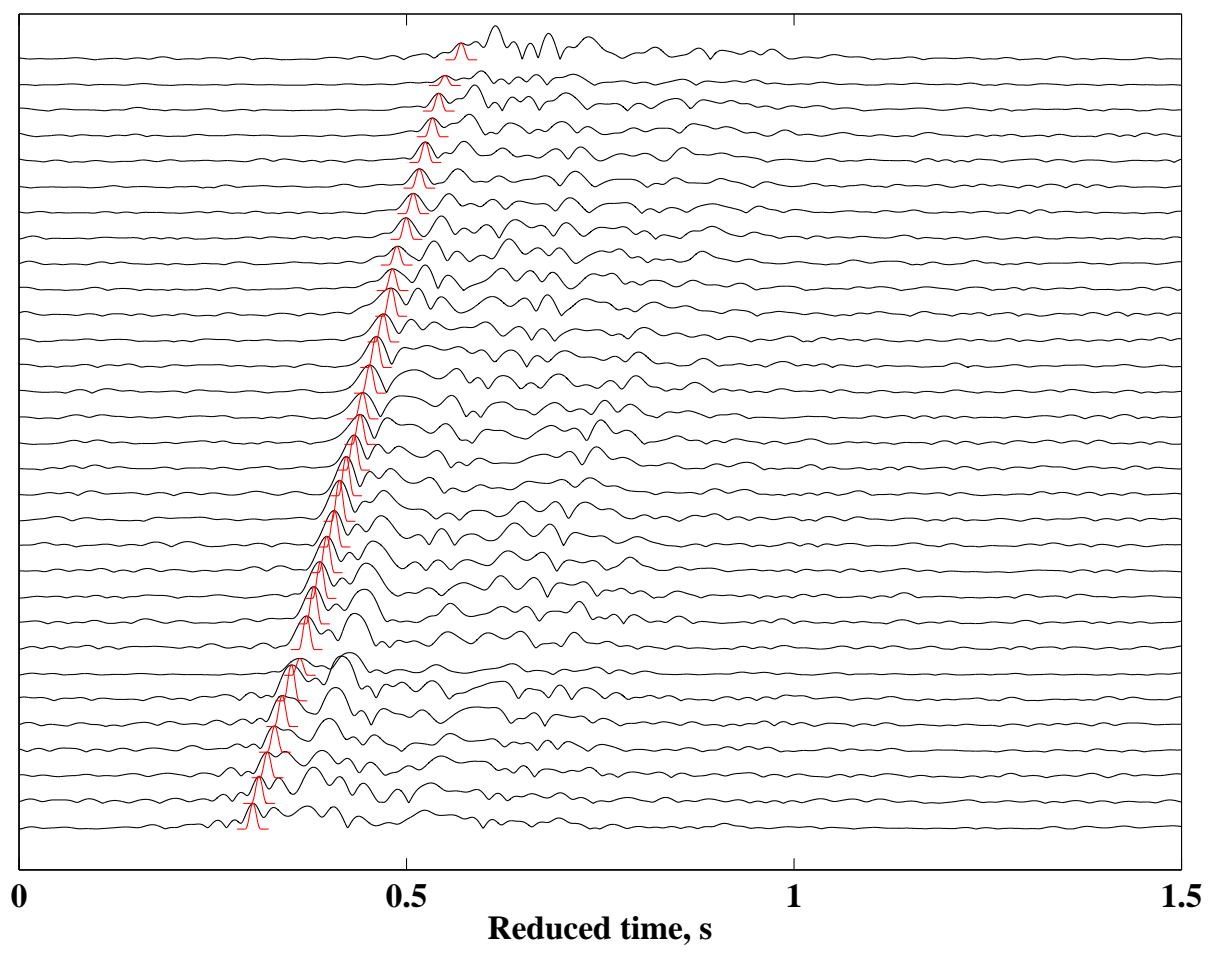

Figure 2-5: Signals received along the HLA and cross-correlated with its replica (compressed pulses) are shown by black lines. Hann windowed outputs are shown by red lines.

in the quiescent periods of internal wave activity. In an independent analysis, Duda et. al. showed [14] similar angle of arrival fluctuations using horizontal coherence analysis along the HLA for the lower frequency $(100 \mathrm{~Hz})$ phase-coded signal radiated by the Miami Sound Machine source that was located not far from the NRL300 (MSM source in Fig. 2-3). Also, Badiey et. al. [15] performed careful modal and frequency decomposition of the NRL300 signal on the August 17, during another time of strong internal wave activity, and registered two distinct arrivals for certain modes, clearly indicating the out of vertical plane acoustic propagation.

The uncertainty in the directional characteristics of the acoustic signals seen in our observations and other known directional studies from SW06 clearly indicate the importance of understanding the three-dimensional acoustic propagation in presence of fine scale oceanography. In the studies which follow, we use both theoretical and computational approaches to summarize both the known effects and also to investigate other possible three-dimensional mechanisms causing out of vertical plane acoustic 


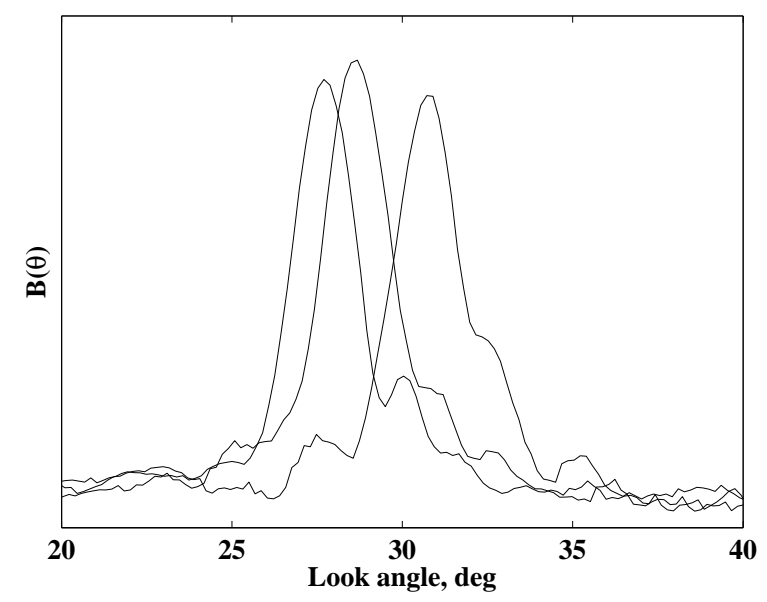

Figure 2-6: Beamforming output for three selected receptions for transmissions during 11:30-11:37 and 12:00-12:07 on August 19, 2006.

propagation through ocean internal waves as well as two other wave types that are also believed to impact the three-dimensional acoustics. 


\section{Chapter 3}

\section{Brief Overview of Normal Mode}

\section{Theory}

There are three common approaches for describing underwater acoustic propagation: rays, normal modes and the parabolic equation. Ray-based theory is constrained by the high frequency approximation, and is most suitable at short distances from the source, where the field is composed of a direct path and a few reflected waves. In shallow water, the wavelength of low frequency signals is often comparable to the water column depth. Also, we are interested in horizontal distances of several to tens of kilometers over which multiple acoustic interactions with both the ocean surface and seabed occur. This makes the normal mode and parabolic equation approaches more suitable for our applications. Parabolic equation algorithms are very efficient methods of computing the acoustic field. However, they don't provide us with the physics insight of the normal mode technique, such as coupling in range dependent environments. In this chapter, we will review the fundamentals of normal mode theory and its applications to shallow water waveguides. To start, we will derive the depth separated normal mode equation for the case of a range independent environment (Section 3.1). For understanding the fundamental features of acoustic propagation in shallow water, we will discuss normal mode solutions for two canonical waveguides: a hard bottom ideal waveguide (Section 3.2), and the Pekeris waveguide, a more complex problem that involves a penetrable acoustic half-space bottom (Section 3.3). In Sec- 
tion 3.4, coupled mode equations will be derived for the waveguides with properties varying in three dimensions.

\subsection{A Point Source in a Range Independent Waveg- uide}

We begin with the formal three-dimensional Helmholtz equation that governs the sound pressure field excited by a point source at coordinates $\left(x_{s}, y_{s}, z_{s}\right)$ :

$$
\rho \nabla\left(\frac{1}{\rho} \nabla p\right)+\frac{\omega^{2}}{c^{2}} p=-\delta\left(x-x_{s}\right) \delta\left(y-y_{s}\right) \delta\left(z-z_{s}\right)
$$

where $p(x, y, z)$ is the acoustic pressure, $\rho(x, y, z)$ is the medium density, $c(x, y, z)$ is the medium sound speed, $\omega=2 \pi f$ is the acoustic angular frequency, and $\delta(x)$ is the Dirac delta function. In this section, we consider constant water depth, and a horizontally stratified medium with sound speed $c(z)$ and density $\rho(z)$. We seek a solution of the homogeneous part of Eq. (3.1) in terms of depth and radial functions $\Psi(z)$ and $\Phi(x, y)$ :

$$
p(x, y, z)=\Phi(x, y) \Psi(z)
$$

By substituting acoustic pressure of the form of Eq. (3.2) into the homogeneous part of Eq. (3.1) and dividing it by $\Phi(x, y) \Psi(z)$, we obtain

$$
\frac{1}{\Psi}\left[\rho \frac{\partial}{\partial z}\left(\frac{1}{\rho} \frac{\partial \Psi}{\partial z}\right)+\frac{\omega^{2}}{c^{2}} \Psi\right]+\frac{1}{\Phi}\left[\frac{\partial^{2} \Phi}{\partial x^{2}}+\frac{\partial^{2} \Phi}{\partial y^{2}}\right]=0
$$

The left hand term in the above equation is a function of $z$ and the right hand term is a function of $x$ and $y$. Therefore, Eq. (3.3) is satisfied if each of the terms is equal to a constant. Denotong this constant by $k_{r m}^{2}$, we obtain vertical equation

$$
\rho(z) \frac{d}{d z}\left(\frac{1}{\rho(z)} \frac{d}{d z} \Psi_{m}(z)\right)+\left(k^{2}(z)-k_{r m}^{2}\right) \Psi_{m}(z)=0
$$


where

$$
k(z) \equiv \sqrt{\frac{\omega^{2}}{c^{2}(z)}}
$$

is the medium wavenumber. Equation (3.4), when used with appropriate boundary conditions on the finite depth interval $0 \leq z \leq z_{\max }$, is a proper Sturm-Liouville problem with a weighting function $\frac{1}{\rho(z)}$. The solutions to a proper Sturm-Liouville problem are the eigenvalues $k_{r m}$ and associated eigenfunctions $\Psi_{m}$ that constitute a complete set. The eigenfunctions are orthonormal in the sense that

$$
\int \frac{\Psi_{n}(z) \Psi_{m}^{*}(z)}{\rho(z)} d z=\delta_{m n}= \begin{cases}1, & m=n \\ 0, & m \neq n\end{cases}
$$

where $\delta_{m n}$ is called Kronecker delta and the star denotes Hermitian conjugate. The eigenfunctions $\Psi_{m}$ are called the normal modes, and the eigenvalues $k_{r m}$ are called the horizontal modal wavenumbers. We also define the vertical modal wavenumber to be

$$
k_{z m}(z)=\sqrt{k^{2}(z)-k_{r m}^{2}}
$$

Vertical equation (Eq. (3.4)) is often called the modal equation. Normal modes $\Psi_{m}$ form a complete set, i.e. any function can be written as a weighted sum of normal modes. Therefore, we write acoustic pressure as

$$
p(x, y, z)=\sum_{m} \Phi_{m}(x, y) \Psi_{m}(z) .
$$

Substituting Eq. (3.8) into Eq. (3.1) yields

$$
\sum_{m=1}^{\infty}\left\{\left[\frac{\partial^{2} \Phi_{m}}{\partial x^{2}}+\frac{\partial^{2} \Phi_{m}}{\partial y^{2}}\right] \Psi_{m}+k_{r m}^{2} \Phi_{m} \Psi_{m}\right\}=-\delta\left(x-x_{s}\right) \delta\left(y-y_{s}\right) \delta\left(z-z_{s}\right)
$$


Applying the operator

$$
\int(\cdot) \frac{\Psi_{n}(z)}{\rho(z)} d z
$$

to both sides of Eq. (3.9), we obtain the separated horizontal equation

$$
\left(\frac{\partial^{2}}{\partial x^{2}}+\frac{\partial^{2}}{\partial y^{2}}\right) \Phi_{n}+k_{r n}^{2} \Phi_{n}=\frac{\Psi_{n}\left(z_{s}\right)}{\rho\left(z_{s}\right)} \delta\left(x-x_{s}\right) \delta\left(y-y_{s}\right),
$$

which is a Bessel's equation of the zeroth order and has solutions

$$
\Phi_{n}(r)=\frac{i H_{0}^{(1)}\left(k_{r n} r\right) \Psi_{n}^{*}\left(z_{s}\right)}{4 \rho\left(z_{s}\right)},
$$

where $H_{0}^{(1)}\left(k_{r n} r\right)$ is the zeroth order Hankel function of the first kind, and

$$
r=\sqrt{\left(x-x_{s}\right)^{2}+\left(y-y_{s}\right)^{2}} .
$$

Equations (3.8) and (3.12) give the following expression for acoustic pressure:

$$
p(r, z)=\frac{i}{4 \rho\left(z_{s}\right)} \sum_{m=1}^{\infty} \Psi_{m}^{*}\left(z_{s}\right) \Psi_{m}(z) H_{0}^{(1)}\left(k_{r m} r\right) .
$$

Using the asymptotic form of the Hankel function for $k_{r m} r>>1$, Eq. (3.14) becomes

$$
p(x, y, z) \approx \frac{e^{i \pi / 4}}{\sqrt{8 \pi r} \rho\left(z_{s}\right)} \sum_{m=1}^{\infty} \Psi_{m}\left(z_{s}\right) \Psi_{m}(z) \frac{e^{i k_{r m} r}}{\sqrt{k_{r m}}} .
$$

\subsection{Normal Modes for an Ideal Waveguide}

Let us consider an example of the simplest shallow water profile, an ideal waveguide with pressure release surface, hard bottom, depth $H_{b o t}$, constant sound speed $c_{0}$ and density $\rho_{0}$ (Fig. 3-1). It can be shown [33] that corresponding normal modes are

$$
\Psi_{m}(z)=\sqrt{\frac{2 \rho_{0}}{H_{b o t}}} \sin k_{z m} z
$$




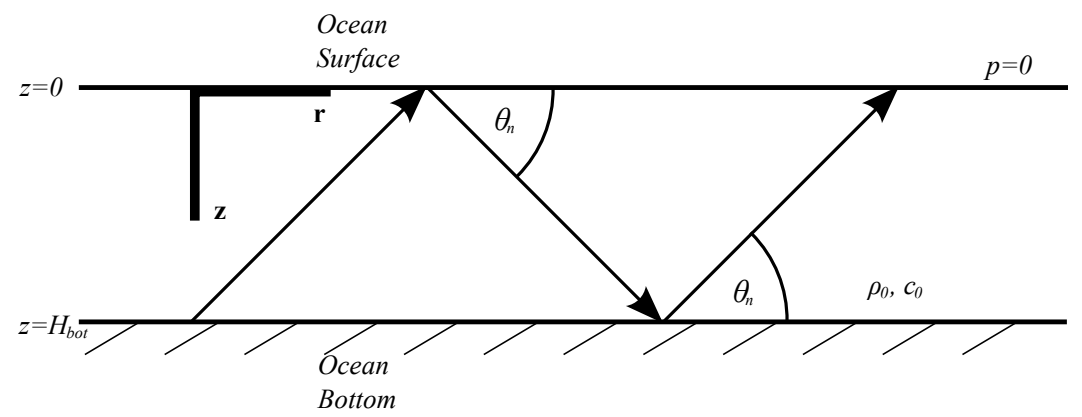

Figure 3-1: A homogeneous fluid layer with pressure release surface and hard bottom (Ideal waveguide).

where the vertical modal wavenumbers are

$$
k_{z m}=\left(n-\frac{1}{2}\right) \frac{\pi}{H}
$$

The horizontal wavenumbers are therefore

$$
k_{z m}=\sqrt{\left(\frac{\omega}{c}\right)^{2}-\left[\left(m-\frac{1}{2}\right) \frac{\pi}{H_{b o t}}\right]^{2}} .
$$

Using Eq. (3.15), the acoustic pressure at a large distance from the source becomes

$$
\begin{aligned}
p(r, z) & \approx \frac{e^{i \pi / 4}}{H_{b o t} \sqrt{2 \pi r}} \sum_{m=1}^{\infty} \sin \left(k_{z m} z_{s}\right) \sin \left(k_{z m} z\right) \frac{e^{i k_{r m} r}}{\sqrt{k_{r m}}} \\
& =\frac{e^{-i \pi / 4}}{H_{b o t} \sqrt{2 \pi r}} \sum_{m=1}^{\infty} \frac{\sin \left(k_{z m} z_{s}\right)}{\sqrt{k_{r m}}}\left[e^{i\left(k_{z m} z+k_{r m} r\right)}-e^{-i\left(k_{z m} z-k_{r m} r\right)}\right] .
\end{aligned}
$$

The two terms in the square brackets of Eq. (3.19) represent down- and upgoing plane waves with vertical grazing angles $\theta_{m}$ defined by the angle between the wavevector and the horizontal plane (Fig. 3-1), such that

$$
k_{r m}=k \cos \theta_{m}, k_{z m}=k \sin \theta_{m}
$$

With increasing mode number, the grazing angle increases, and therefore over a fixed distance in range, higher order modes experience more bottom and surface interactions.

Although there is an infinite number of normal modes for this waveguide, only a 


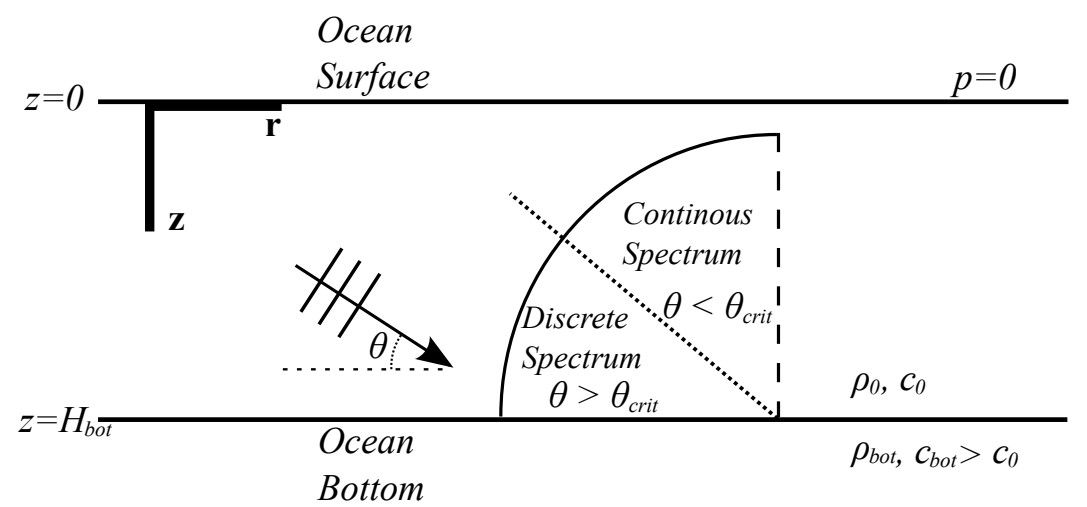

Figure 3-2: Homogeneous fluid layer with a pressure release surface and a homogeneous, higher sound speed, bottom half space (Pekeris waveguide); vertical grazing angle regions are for the discrete and continous modal spectrum.

finite number of them have a real horizontal wavenumber. These modes are called propagating modes. According to Eq. (3.18), for

$$
m>M=\frac{w H_{b o t}}{c_{0} \pi}+\frac{1}{2},
$$

the horizontal wavenumber becomes imaginary, and the modal amplitude decays exponentially with range. The corresponding modes are called evanescent modes. Thus, for large distances from the source, one can limit the sum in Eqs. (3.19) and (3.15) by $M$.

\subsection{Normal Modes for Pekeris Waveguide}

When deriving Eq. (3.14), we made an assumption of a non-singular Sturm-Liouville problem that has a complete set of normal modes. Many ocean-acoustic problems, however, have a mixed wavenumber spectrum composed of a discrete finite set of normal modes that are associated with a properly-posed Sturm-Liouville problem, and continuous part associated with an improperly-posed Sturm-Liouville problem. One of the canonical shallow water waveguide examples that has a mixed spectrum is the Pekeris waveguide. In this waveguide model, a homogeneous fluid layer with depth $H_{b o t}$, sound speed $c_{0}$, density $\rho_{0}$, and a pressure release surface overlies a homogeneous bottom fluid half space with higher velocity $c_{b o t}$ and density $\rho_{\text {bot }}$ (Fig. 
3-2). The Pekeris waveguide is one of the fundamental problems in ocean acoustics, because it describes a large number of the acoustic propagation properties in shallow water.

We define the angle between the plane wave wavevector and the horizontal plane as a vertical grazing angle (Fig. 3-2), similar to Eq. (3.20). Unlike the previous ideal waveguide, acoustic reflection from the bottom interface is not always total, and the reflection coefficient $R_{b o t}$ depends on the vertical grazing angle:

$$
R_{b o t}(\theta)=\frac{\rho_{b o t} c_{b o t} / \sin \theta_{b o t}-\rho_{0} c_{0} / \sin \theta}{\rho_{b o t} c_{b o t} / \sin \theta_{b o t}+\rho_{0} c_{0} / \sin \theta}
$$

where $\theta_{b o t}$ is the vertical grazing angle of the plane wave transmitted into the bottom half space. It is related to the water layer grazing angle through Snell's law:

$$
c_{b o t} \cos \theta=c_{0} \cos \theta_{b o t}
$$

There are two distinct vertical grazing angle regions that determine behavior of $R_{b o t}$. These are

$$
\begin{aligned}
& \left|R_{\text {bot }}\right|<1, \quad \theta>\theta_{\text {crit }} \\
& \left|R_{\text {bot }}\right|=1, \quad \theta \leq \theta_{\text {crit }}
\end{aligned}
$$

where

$$
\theta_{\text {crit }}=\cos ^{-1}\left(\frac{c_{0}}{c_{b o t}}\right)
$$

is the critical grazing angle.

For $\theta<\theta_{\text {crit }}$, total internal reflection occurs and therefore, Eq. (3.4), together with the free surface condition and the Neumann boundary condition at the bottom interface, is associated with a proper Sturm-Liouville problem that has a finite discrete set of perfectly trapped modes. At steeper grazing angles, $\theta>\theta_{\text {crit }}$, the acoustic energy is no longer perfectly reflected, and leakage into the bottom half space occurs. The Sturm-Liouville problem becomes improperly posed, and this corresponds to a 
modal continuum (See [34] for details). Acoustic pressure is formally expressed as the sum of a trapped modes contribution (discrete part) and a contribution from the modal continuum (continuous part):

$$
p(r, z)=p_{d}(r, z)+p_{c}(r, z) .
$$

The modal continuum is associated with energy loss and thus its contribution to the acoustic pressure decays with distance from the source. Therefore, at sufficiently large distances from the source in a range independent environment or in an environment with slowly varying parameters (see next section), its contribution is negligible, and we can approximate an acoustic field by including only the first term in Eq. (3.26). The continuous part of the pressure spectrum becomes important when either the distance from the source or the distance at which significant changes to the environmental parameters occur, are less than several wavelengths.

The normal modes for the Pekeris waveguide are [34]

$$
\Psi_{m}(z)= \begin{cases}A_{m} \sin \left(k_{z m} z\right), & 0 \leq z \leq H_{b o t} \\ A_{m} \sin \left(k_{z m} H_{b o t}\right) e^{i k_{z m, b o t}\left(z-H_{b o t}\right)}, & z \geq H_{b o t}\end{cases}
$$

where the vertical modal wavenumbers in the layer, $k_{z m}$, and in the bottom half space, $k_{z m, b o t}$, are related to the horizontal modal wavenumber by

$$
\begin{aligned}
k_{z m} & =\sqrt{\left(\frac{\omega}{c_{0}}\right)^{2}-k_{r m}^{2}}, \\
k_{z m, b o t} & =\sqrt{\left(\frac{\omega}{c_{b o t}}\right)^{2}-k_{r m}^{2}}
\end{aligned}
$$

and are found from

$$
\tan \left(k_{z m} H_{b o t}\right)=-\frac{\rho_{b o t} k_{z m}}{\rho_{0} k_{z m, b o t}} .
$$

Equation (3.30) is a transcendental equation for the eigenvalues $k_{r m}(\omega)$. Using the 
orthonormality of the modes defined by Eq. (3.6), it is found that

$$
A_{m}=\sqrt{2}\left[\frac{1}{\rho_{0}}\left(H_{b o t}-\frac{\sin \left(2 k_{z m} H_{b o t}\right)}{2 k_{z m}}\right)-\frac{1}{\rho_{b o t}} \frac{\sin ^{2}\left(k_{z m} H_{b o t}\right)}{i k_{z m, b o t}}\right] .
$$

With Eq. (3.15), we can approximate the acoustic pressure field at large distances from the acoustic source as

$$
p(r, z) \approx \begin{cases}\frac{e^{i \pi / 4}}{\sqrt{8 \pi r} \rho_{0}} \sum_{m=1}^{M} A_{m}^{2} \sin \left(k_{z m} z_{s}\right) \sin \left(k_{z m} z\right) \frac{e^{i k_{r m} r}}{\sqrt{k_{r m}}}, & 0 \leq z \leq H_{b o t}, \\ \frac{e^{i \pi / 4}}{\sqrt{8 \pi r} \rho_{0}} \sum_{m=1}^{M} A_{m}^{2} \sin \left(k_{z m} z_{s}\right) \sin \left(k_{z m} H_{b o t}\right) e^{i k_{z m, b o t}\left(z-H_{b o t}\right)} \frac{e^{i k_{r m} r}}{\sqrt{k_{r m}}}, & z \geq H_{b o t},\end{cases}
$$

where $M$ denotes the number of trapped modes.

\subsection{Normal Modes for Range Dependent Environ- ments}

Let us return to the Helmholtz equation (Eq. (3.1)) and rewrite it in its Cartesian components,

$$
\rho \frac{\partial}{\partial x}\left[\frac{1}{\rho} \frac{\partial p}{\partial x}\right]+\rho \frac{\partial}{\partial y}\left[\frac{1}{\rho} \frac{\partial p}{\partial y}\right]+\rho \frac{\partial}{\partial z}\left[\frac{1}{\rho} \frac{\partial p}{\partial z}\right]+\frac{\omega^{2}}{c^{2}} p=-\delta\left(x-x_{s}\right) \delta\left(y-y_{s}\right) \delta\left(z-z_{s}\right) .
$$

Using the completeness of the normal mode set, we seek a solution of Eq. (3.33) as a sum of local modes,

$$
p(x, y, z)=\sum_{m} \Phi_{m}(x, y) \Psi_{m}(x, y, z),
$$

where $\Phi_{m}(x, y)$ are the complex modal amplitudes and $\Psi_{m}(x, y, z)$ are the local normal modes defined by

$$
\rho(x, y, z) \frac{\partial}{\partial z}\left[\frac{1}{\rho(x, y, z)} \frac{\partial \Psi_{m}(x, y, z)}{\partial z}\right]+\left[\frac{\omega^{2}}{c^{2}(x, y, z)}-k_{r m}^{2}(x, y)\right] \Psi_{m}(x, y, z)=0 .
$$


and appropriate boundary conditions at the sea surface and bottom. The local normal mode set is therefore found at each point $(x, y)$ in the horizontal. Substituting Eq. (3.35) into Eq. (3.33), applying the operator

$$
\int(\cdot) \frac{\Psi_{n}(x, y, z)}{\rho(x, y, z)} d z
$$

and using the orthonormality of the modes (Eq. (3.6)), we get the following equation for modal amplitudes

$$
\begin{aligned}
\frac{\partial^{2} \Phi_{n}}{\partial x^{2}}+ & \frac{\partial^{2} \Phi_{n}}{\partial y^{2}}+k_{r n}^{2}(x, y) \Phi_{n}+\sum_{m} A_{m n} \Phi_{m}+ \\
& \sum_{m} B_{m n} \frac{\partial \Phi_{m}}{\partial x}+\sum_{m} C_{m n} \frac{\partial \Phi_{m}}{\partial y}=-\delta\left(x-x_{s}\right) \delta\left(y-y_{s}\right) \frac{\Psi_{n}\left(x, y, z_{s}\right)}{\rho\left(x, y, z_{s}\right)},
\end{aligned}
$$

where the coupling coefficients $A_{m n}, B_{m n}, C_{m n}$ are defined by

$$
\begin{gathered}
A_{m n}=\int\left(\rho \frac{\partial}{\partial x}\left[\frac{1}{\rho}\right] \frac{\partial \Psi_{m}}{\partial x}+\rho \frac{\partial}{\partial y}\left[\frac{1}{\rho}\right] \frac{\partial \Psi_{m}}{\partial y}\right) \frac{\Psi_{n}}{\rho} d z \\
B_{m n}=\int\left(2 \frac{\partial \Psi_{m}}{\partial x}+\rho \frac{\partial}{\partial x}\left[\frac{1}{\rho}\right] \Psi_{m}\right) \frac{\Psi_{n}}{\rho} d z
\end{gathered}
$$

and

$$
C_{m n}=\int\left(2 \frac{\partial \Psi_{m}}{\partial y}+\rho \frac{\partial}{\partial y}\left[\frac{1}{\rho}\right] \Psi_{m}\right) \frac{\Psi_{n}}{\rho} d z
$$

For environments whose parameters change in the horizontal slowly compared to the acoustic wavelength scale, the coupling coefficients are negligible, and the equation for the modal amplitude becomes

$$
\frac{\partial^{2} \Phi_{m}}{\partial x^{2}}+\frac{\partial^{2} \Phi_{m}}{\partial y^{2}}+k_{r m}^{2}(x, y) \Phi_{m}=-\delta\left(x-x_{s}\right) \delta\left(y-y_{s}\right) \frac{\Psi_{m}\left(x, y, z_{s}\right)}{\rho\left(x, y, z_{s}\right)}
$$

often called the horizontal refraction equation [33]. This approximation, also called the adiabatic approximation was originally introduced for cylindrically symmetric 
range dependent waveguides by Pierce [35], and more formal derivations were obtained in [36]. Using Eq. (3.41), our initial three-dimensional problem of Eq. (3.33) reduces to finding the local normal mode set for each point in the horizontal followed by solving several two-dimensional problems of Eq. (3.41), one for each normal mode. This approximation is a very powerful tool as it allows us to solve a number of three-dimensional acoustic problems analytically and considerably reduces the computational time of numerical modeling.

Coastal waters are often associated with considerable changes of waveguide properties over short distances, and generally speaking, mode coupling techniques need to be employed. However, as we will see in Chapter 5, even with presence of strong nonlinear internal waves or bottom waves of large amplitude, the adiabatic approximation is sufficient for acoustic propagation within the narrow directional ranges centered in the direction of the ocean wave crests. 


\section{Chapter 4}

\section{Acoustic Normal Mode Perturbations due to Ocean \\ Internal Waves, Bottom Sediment Waves and Surface Waves.}

To understand the physics of three-dimensional shallow water acoustic propagation in the presence of internal waves, bottom sediment waves, or ocean surface waves, it is important to know the impact of each of these effects on normal mode properties. In this chapter, we will investigate the perturbation of the normal mode horizontal wavenumbers and their corresponding mode functions due to the presence of waves of three types. In order to do this, we will introduce a simple idealized model of a background shallow water column and then derive the governing equation for the modal wavenumbers (Section 4.1). Each of the three types of waves will be treated as a corresponding modification of the model that allows us to find the perturbation to the modal wavenumbers analytically (Sections $4.2-4.4$ ). 


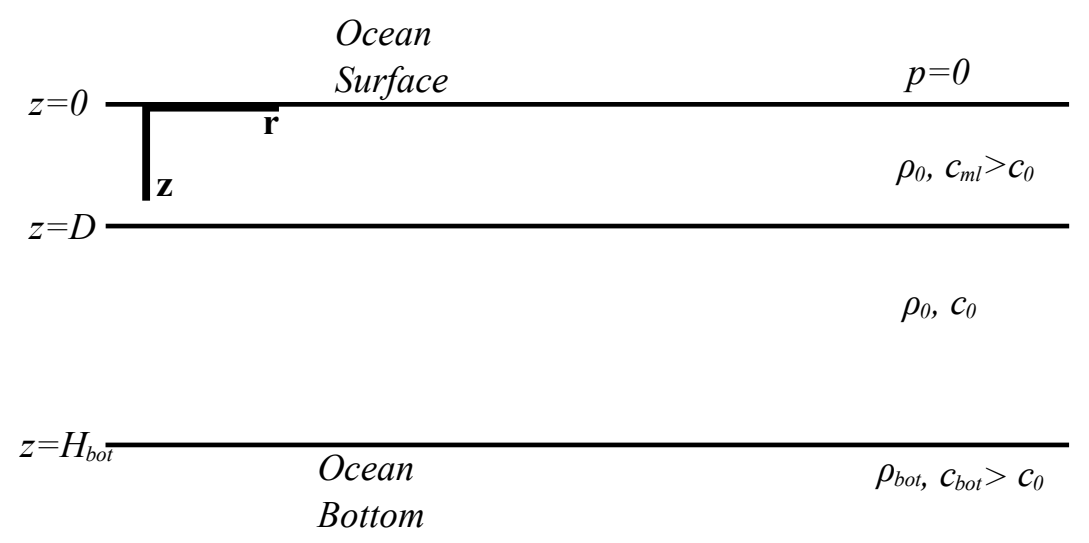

Figure 4-1: Background water column model: two homogeneous fluid layers (upper layer having slightly higher sound speed) bounded by a free surface and a homogeneous half space bottom of higher sound speed.

\subsection{Waveguide Model}

Let us consider an idealized waveguide with two horizontal fluid layers bounded by a vacuum half space on top and by a fluid half space below (Fig. 4-1). The lower acoustic layer is characterized by a constant reference sound speed and density in the water $c_{0}$, and $\rho_{0}$ respectively. The upper layer, that is often called the mixed layer, has the same density, but slightly higher than reference sound speed $c_{m l}=c_{0}+\Delta c$. The bottom half space has a constant density and sound speed equal to the seabed reference values $c_{b o t}$ and $\rho_{b o t}$. The depth of the interface between layers is $D$ and the a bottom depth is $H_{b o t}$. Although this model does not exactly describe the realistic vertical variation of sound speed, it is fairly simple, and at the same time approximates a wide range of shallow water columns, especially in the summer time or in low latitudes, when a higher sound speed surface mixed layer exists.

We assume our waveguide model to be range independent, and derive the expressions for the normal modes, together with a transcendental equation, similar to derivations for the Pekeris waveguide [34]. The general solution of Eq. (3.4) within 
the two layers and the bottom half space is

$$
\Psi_{m}(z)= \begin{cases}C_{m} \sin \left(k_{z m, m l} z\right), & 0 \leq z \leq D \\ A_{m} e^{i k_{z m} z}+B_{m} e^{-i k_{z m} z}, & D \leq z \leq H_{b o t} \\ D_{m} e^{i k_{z m, b o t} z}, & z \geq H_{b o t},\end{cases}
$$

where we have applied the free surface boundary condition for the upper layer and the Sommerfeld radiation condition for the bottom half space. $A_{m}, B_{m}, C_{m}$, and $D_{m}$ are the constants that satisfy continuity of pressure and particle velocity at the interface between layers,

$$
\begin{gathered}
\Psi_{m}\left(D^{+}\right)=\Psi_{m}\left(D^{-}\right), \\
\frac{\partial \Psi_{m}\left(D^{+}\right)}{\partial z}=\frac{\partial \Psi_{m}\left(D^{-}\right)}{\partial z},
\end{gathered}
$$

and between the lower layer and bottom half space,

$$
\begin{gathered}
\Psi_{m}\left(H_{b o t}^{+}\right)=\Psi_{m}\left(H_{b o t}^{-}\right) \\
\frac{1}{\rho_{b o t}} \frac{\partial \Psi_{m}\left(H_{b o t}^{+}\right)}{\partial z}=\frac{1}{\rho_{0}} \frac{\partial \Psi_{m}\left(H_{b o t}^{-}\right)}{\partial z} .
\end{gathered}
$$

Vertical modal wavenumbers in the lower layer, $k_{z m}$, and bottom half space, $k_{z m, b o t}$, are defined in the same manner as for the Pekeris waveguide by Eqs. (3.28)-(3.29) and

$$
k_{z m, m l}=\sqrt{\frac{\omega^{2}}{c_{m l}^{2}}-k_{r m}^{2}}
$$

is the vertical modal wavenumber in the mixed layer. The boundary conditions (4.2) 
- (4.5) can be rewritten as

$$
\begin{aligned}
C_{m} \sin \left(k_{z m, m l} D\right) & =A_{m} e^{i k_{z m} D}+B_{m} e^{-i k_{z m} D} \\
C_{m} \cos \left(k_{z m, m l} D\right) & =\frac{i k_{z m}}{k_{z m, m l}}\left(A_{m} e^{i k_{z m} D}-B_{m} e^{-i k_{z m} D}\right) \\
D_{m} e^{i k_{z m, b o t} H_{b o t}} & =A_{m} e^{i k_{z m} H_{b o t}}+B_{m} e^{-i k_{z m} H_{b o t}} \\
\frac{1}{\rho_{b o t}} D_{m} e^{i k_{z m, b o t} H_{b o t}} & =\frac{k_{z m}}{\rho_{0} k_{z m, b o t}}\left(A_{m} e^{i k_{z m} H_{b o t}}-B_{m} e^{-i k_{z m} H_{b o t}}\right) .
\end{aligned}
$$

From Eqs. (4.7) and (4.8), it follows that

$$
B_{m}=A_{m} e^{2 i k_{z m} D} \alpha_{m}
$$

where $\alpha_{m}$ is

$$
\alpha_{m}=\frac{i \tan \left(k_{z m, m l} D\right) k_{z m}-k_{z m, m l}}{i \tan \left(k_{z m, m l} D\right) k_{z m}+k_{z m, m l}} .
$$

From Eqs. (4.7) and (4.8) we get

$$
\tan k_{z m} H_{b o t}=i \frac{\left(\rho_{b o t} k_{z m}-\rho_{0} k_{z m, b o t}\right) A_{m}-\left(\rho_{b o t} k_{z m}+\rho_{0} k_{z m, b o t}\right) B_{m}}{\left(\rho_{b o t} k_{z m}-\rho_{0} k_{z m, b o t}\right) A_{m}+\left(\rho_{b o t} k_{z m}+\rho_{0} k_{z m, b o t}\right) B_{m}} .
$$

Substituting Eq. (4.11) into Eq. (4.13) gives us

$$
\begin{aligned}
& \tan \left(k_{z m} H_{b o t}\right)= \\
& i \frac{\left(\rho_{b o t} k_{z m}-\rho_{0} k_{z m, b o t}\right)\left(i \tan \left(k_{z m, m l} D\right) k_{z m}+k_{z m, m l}\right)-\left(\rho_{b o t} k_{z m}+\rho_{0} k_{z m, b o t}\right) e^{2 i k_{z m} D}\left(i \tan \left(k_{z m, m l} D\right) k_{z m}-k_{z m, m l}\right)}{\left(\rho_{b o t} k_{z m}-\rho_{0} k_{z m, b o t}\right)\left(i \tan \left(k_{z m, m l} D\right) k_{z m}+k_{z m, m l}\right)+\left(\rho_{b o t} k_{z m}+\rho_{0} k_{z m, b o t}\right) e^{2 i k_{z m} D}\left(i \tan \left(k_{z m, m l} D\right) k_{z m}-k_{z m, m l}\right)}
\end{aligned}
$$

Equation (4.14) is a transcendental equation for our model. Note that it reduces to the standard transcendental equation for the Pekeris waveguide (Eq. (3.30)) by setting the depth of the upper acoustic layer to zero, i.e. $D=0$, or by making the vertical modal wavenumbers in the layers equal, i.e. $k_{z m, m l}=k_{z m}$. Normal modes 

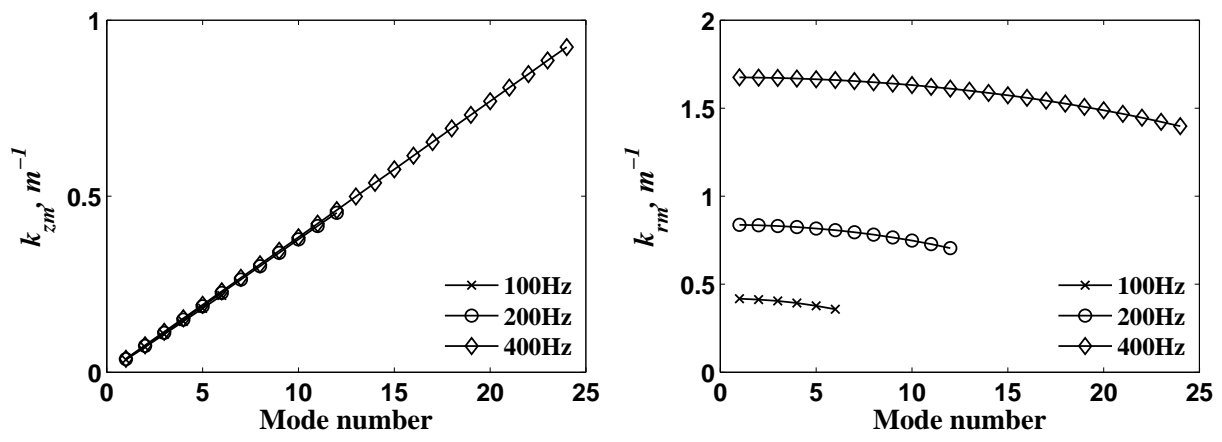

Figure 4-2: Vertical modal wavenumber for the lower layer (left), and horizontal modal wavenumber (right) computed with the KRAKEN normal mode code for the waveguide model having sound speed in upper and lower layers $c_{m l}=1530 \mathrm{~m} / \mathrm{s}$ and $c_{0}=1500 \mathrm{~m} / \mathrm{s}$ respectively, density $\rho_{0}=1000 \mathrm{~kg} / \mathrm{m}^{3}$, depth of the interface between water layers $D=15 \mathrm{~m}$, and bottom of depth $H_{b o t}=80 \mathrm{~m}$ with sound speed $c_{b o t}=1800 \mathrm{~m} / \mathrm{s}$ and density $\rho_{b o t}=2000 \mathrm{~kg} / \mathrm{m}^{3}$.

can be expressed through constants $A_{m}$ as

$$
\Psi_{m}(z)= \begin{cases}A_{m} e^{i k_{z m} z}\left(1+\alpha_{m} e^{2 i k_{z m}(D-z)}\right), & 0 \leq z \leq D, \\ \frac{A_{m}}{\sin \left(k_{z m, m l} D\right)} e^{i k_{z m} D}\left(1+\alpha_{m}\right) \sin \left(k_{z m, m l} z\right), & D \leq z \leq H_{b o t} \\ A_{m} e^{-i k_{z m} H_{b o t}}\left(e^{2 i k_{z m} H_{b o t}}+\alpha_{m} e^{2 i k_{z m} D}\right) e^{2 i k_{z m, b o t}(z-H)}, & z \geq H_{b o t}\end{cases}
$$

Finally, by applying normal mode normalization defined by Eq. (3.6), the constants $A_{m}$ are found to be

$$
\begin{aligned}
A_{m} & =\left[\frac{D\left|1+\alpha_{m}\right|^{2}\left(1-\frac{\sin \left(2 k_{z m, m l} D\right)}{2 k_{z m, m l} D}\right)}{2 \rho_{0} \sin ^{2}\left(k_{z m, m l} D\right)}+\right. \\
& \left.\frac{2\left(H_{b o t}-D\right)-\operatorname{Re}\left\{\frac{\alpha_{m} e^{2 i k_{z m}\left(D-H_{b o t}\right)}}{i k_{z m}}\right\}}{\rho_{0}}+\frac{i\left|e^{2 i k_{z m} H_{b o t}}+\alpha_{m} e^{2 i k_{z m} D}\right|^{2}}{2 \rho_{b o t} k_{z m, b o t}}\right]^{-1 / 2} .
\end{aligned}
$$

As an example, we will consider the background waveguide model with bottom depth $H_{b o t}=80 \mathrm{~m}$, lower water layer sound speed $c_{0}=1500 \mathrm{~m} / \mathrm{s}$, water density $\rho_{0}=1000$ $\mathrm{kg} / \mathrm{m}^{3}$, the bottom with sound speed $c_{\text {bot }}=1800 \mathrm{~m} / \mathrm{s}$ and density $\rho_{\text {bot }}=2000 \mathrm{~kg} / \mathrm{m}^{3}$, and the mixed layer with depth $D=15 \mathrm{~m}$ and sound speed $c_{m l}=1530 \mathrm{~m} / \mathrm{s}$. This set of parameters roughly represents the mean water column properties for the along shelf acoustic path in the SW06 experiment. Figure 4-2 shows the horizontal wavenum- 

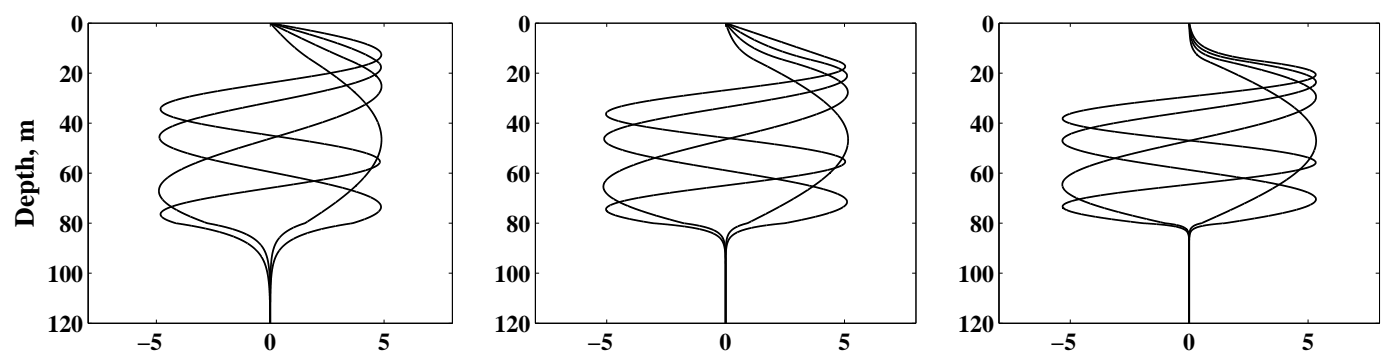

Figure 4-3: First four normal modes for the background water column at $100 \mathrm{~Hz}$ (left), $200 \mathrm{~Hz}$ (middle), and $400 \mathrm{~Hz}$ (right).

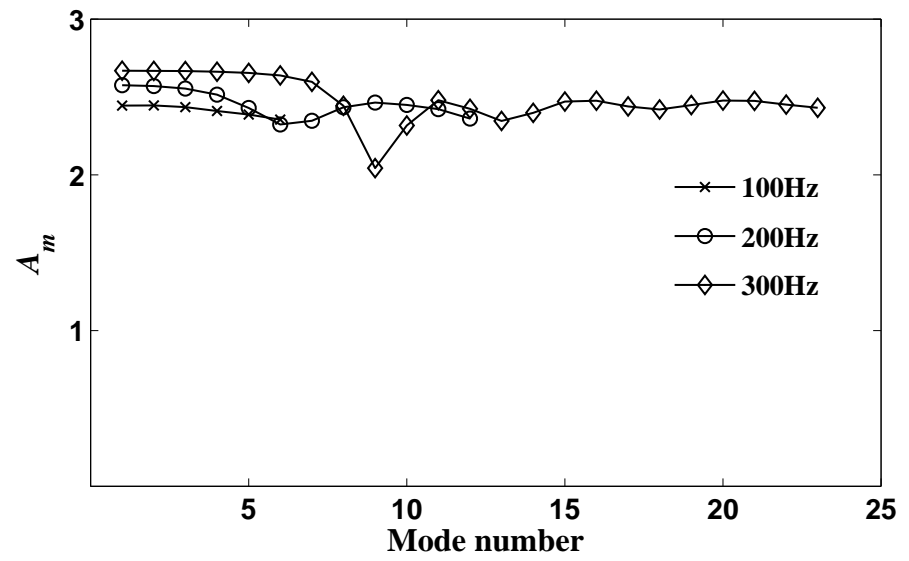

Figure 4-4: Modal normalization constant $A_{m}$ for the background waveguide at frequencies $100 \mathrm{~Hz}, 200 \mathrm{~Hz}$ and $400 \mathrm{~Hz}$. 


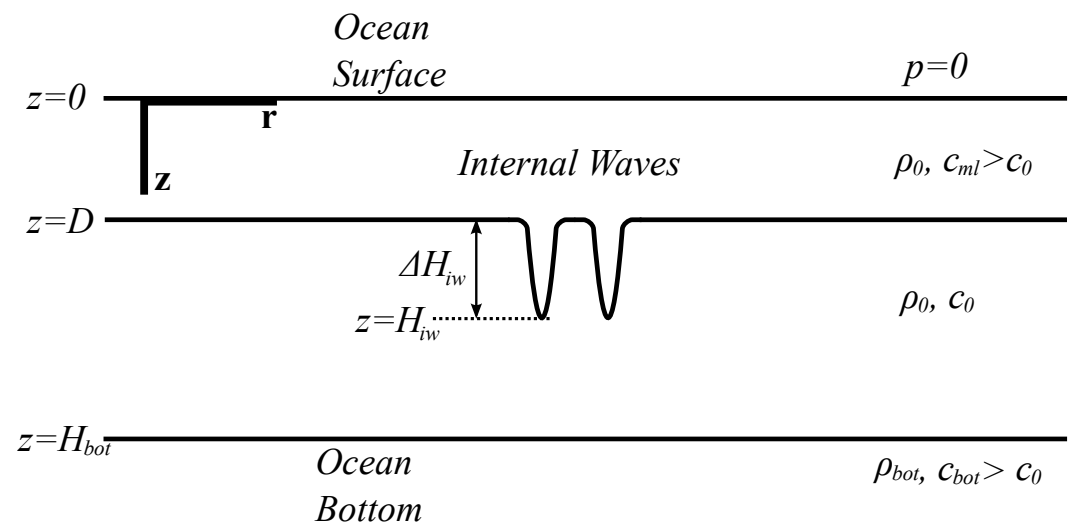

Figure 4-5: Depression internal waves represented by lowering the interface between water layers.

bers $k_{r m}$ for this environment model calculated with acoustic normal mode program KRAKEN [32], for frequencies $100 \mathrm{~Hz}, 200 \mathrm{~Hz}$ and $400 \mathrm{~Hz}$. Similarly to the ideal waveguide, the vertical wavenumber shows almost an linearly increasing dependence on mode number, whereas horizontal wavenumber monotonically decreases with mode number. Once the eigenvalues $k_{r m}$ are found, the corresponding normal mode functions $\Psi_{m}(z)$ are constructed using Eqs. (4.15-4.16). The first four modes for the waveguide given the above parameters are plotted in Fig. 4-3.

It is seen numerically that for the mixed layer depth small compared to the water column depth and in the frequency range 0-500 $\mathrm{Hz}$, the values of $A_{m}$ don't vary significantly across mode number and are approximately equal to $\left[2\left(H_{b o t}-D\right) / \rho_{0}\right]^{-1 / 2}$. As an example, Fig. 4-4 illustrates the values for the frequencies of $100 \mathrm{~Hz}, 200 \mathrm{~Hz}$ and $400 \mathrm{~Hz}$.

\subsection{Internal Waves}

The ocean surface mixed layer, which often has a slightly higher temperature and associated sound speed due to solar heat absorption, is an inherent feature of the continental shelf water column in summer time and in low latitudes. Internal waves, commonly observed on large parts of the continental shelf, displace the thermocline, resulting in a change of the effective depth of the mixed layer. As an approximation to the vertical thermocline displacement, we use the idealized waveguide described 
in the previous section and model the internal waves as a depression of the interface between layers with an amplitude of $\Delta H_{i w}$ (Fig. 4-5). In this section, we will evaluate the amplitude of the modal horizontal wavenumber perturbation due to the presence of these waves. We will employ standard normal mode perturbation theory for the water column fluctuations [37].

Let us define the horizontal modal wavenumber at the wave crest as

$$
k_{r m}^{1}=k_{r m}+\Delta k_{r m}
$$

where $k_{r m}$ is the wavenumber for the unperturbed environment. The corresponding vertical modal wavenumbers for the mixed layer, lower layer, and bottom half space are therefore

$$
\begin{aligned}
k_{z m, m l}^{1} & =\sqrt{\left(\frac{\omega}{c_{m l}}\right)^{2}-k_{r m}^{1}{ }^{2}}, \\
k_{z m}^{1} & =\sqrt{\left(\frac{\omega}{c_{0}}\right)^{2}-k_{r m}^{1}{ }^{2}}, \\
k_{z m, b o t}^{1} & =\sqrt{\left(\frac{\omega}{c_{b o t}}\right)^{2}-k_{r m}^{1}{ }^{2}}
\end{aligned}
$$

respectively. Assuming small changes in water column sound speed, the wavenumber correction $\Delta k_{r m}$ can be expressed in terms of its background values and background normal modes as

$$
\Delta k_{r m}=\frac{1}{2 k_{r m}} \int_{0}^{\infty} \frac{\Delta q(z)\left|\Psi_{m}(z)\right|^{2} d z}{\rho(z)}
$$

The new set of normal modes is

$$
\Psi_{m}^{1}=\Psi_{m}+\sum_{m} a_{m n} \Psi_{m}
$$

where the coefficients $a_{m n}$ are

$$
a_{m n}=\frac{1}{\left(k_{r m}^{2}-k_{r n}^{2}\right)} \int_{0}^{\infty} \frac{\Delta q(z) \Psi_{m}(z) \Psi_{n}(z) d z}{\rho(z)},
$$


and

$$
q(z)=\frac{\omega^{2}}{c^{2}(z)}
$$

In our model, we are considering a displacement of the interface between layers from depth $z=D$ to $z=H_{i w}$. Therefore,

$$
\Delta q(z)= \begin{cases}\frac{\omega^{2}}{c_{m l}^{2}}-\frac{\omega^{2}}{c_{0}^{2}} & , \quad 0 \leq z \leq H_{i w} \\ 0 & , \quad \text { otherwise }\end{cases}
$$

For a small sound speed difference between upper and lower layers, $\Delta c$, we will use its linearized form,

$$
\Delta q(z) \approx \begin{cases}-\frac{2 \omega^{2}}{c_{0}^{3}} \Delta c & , \quad 0 \leq z \leq H_{i w} \\ 0 & , \quad \text { otherwise }\end{cases}
$$

where we have defined

$$
\Delta c=c_{m l}-c_{0}
$$

Equation (4.21) now becomes

$$
\Delta k_{r m} \approx-\frac{\omega^{2} \Delta c}{k_{r m} c_{0}^{3} \rho_{0}} \int_{D}^{H_{i w}}\left|\Psi_{m}(z)\right|^{2} d z
$$

The above integral is easily solved analytically using the normal mode functions defined by Eq. (4.15). The resultant first order contribution to the horizontal modal wavenumber is

$$
\Delta k_{r m} \approx-2 \frac{\left|A_{m}\right|^{2} \omega^{2} \Delta c}{k_{r m} c_{0}^{3} \rho_{0}}\left[\Delta H_{i w}+\frac{\sin \left(k_{z m} \Delta H_{i w}\right) \cos \left(2 \tan ^{-1}\left[\frac{k_{z m, m l}}{k_{z m} \tan k_{z m, m l} D}\right]+k_{z m} \Delta H_{i w}\right)}{k_{z m}}\right]
$$

with normalization constants $A_{m}$ determined by Eqs. (4.16) and (4.12). As we have discussed in the previous section, these constants don't vary significantly across mode 
number. Higher order modes, having steeper vertical grazing angles, are characterized by smaller horizontal wavenumber (see Fig. 4-2). This makes the factor in the left part of the square brackets in Eq. (4.29) slightly increase with mode number. Inside the brackets, the left term is a constant, whereas the right term has oscillatory behavior. The amplitude of its oscillations is inversely proportional to the vertical modal wavenumber $k_{z m}$. We noticed in the previous section that $k_{z m}$ is small for low mode numbers. Therefore, the contribution of the right oscillating term to the total wavenumber correction for these modes is significant. One can note that in the limit $k_{z m} \rightarrow 0$, the right and left terms exactly cancel each other, resulting in the first mode having the smallest perturbation of the horizontal wavenumber. With mode number increasing, $k_{z m}$ becomes greater, and the amplitude of oscillations of the right term becomes smaller. This suggests convergence at high mode numbers of the total wavenumber correction to

$$
\Delta k_{r m} \approx-\left.2 \frac{\left|A_{m}\right|^{2} \omega^{2} \Delta c}{k_{r m} c_{0}^{3} \rho_{0}}\right|_{m=M} \Delta H_{i w}
$$

where $M$ is the maximum number of propagating modes as before.

As an example, we consider our waveguide model and set the maximum depth of the perturbed interface between layers to thirty meters $\left(H_{i w}=30 \mathrm{~m}\right)$. This makes an internal wave of amplitude fifteen meters $\left(\Delta H_{i w}=15 \mathrm{~m}\right)$, which is a typical value observed at a number of continental shelf regions, including the Shallow Water 2006 Experiment site. Figure 4-6 illustrates the change in the horizontal wavenumber for a frequency $200 \mathrm{~Hz}$ caused by this wave, computed with Eq. (4.29) (dashed line), compared with the numerical solution using the KRAKEN normal mode code (solid line). Our approximate solution shows very reasonable agreement with the numerically computed wavenumber correction values. Both curves show oscillations with decreasing amplitude as the mode number increases. As mentioned, the wavenumber correction for the high mode numbers converges to $-3 \mathrm{~m}^{-1}$, as predicted by Eq. (4.30).

Although predicted and numerically computed wavenumber corrections have similar behavior, there is an offset between them which is most noticeable for modes 1 


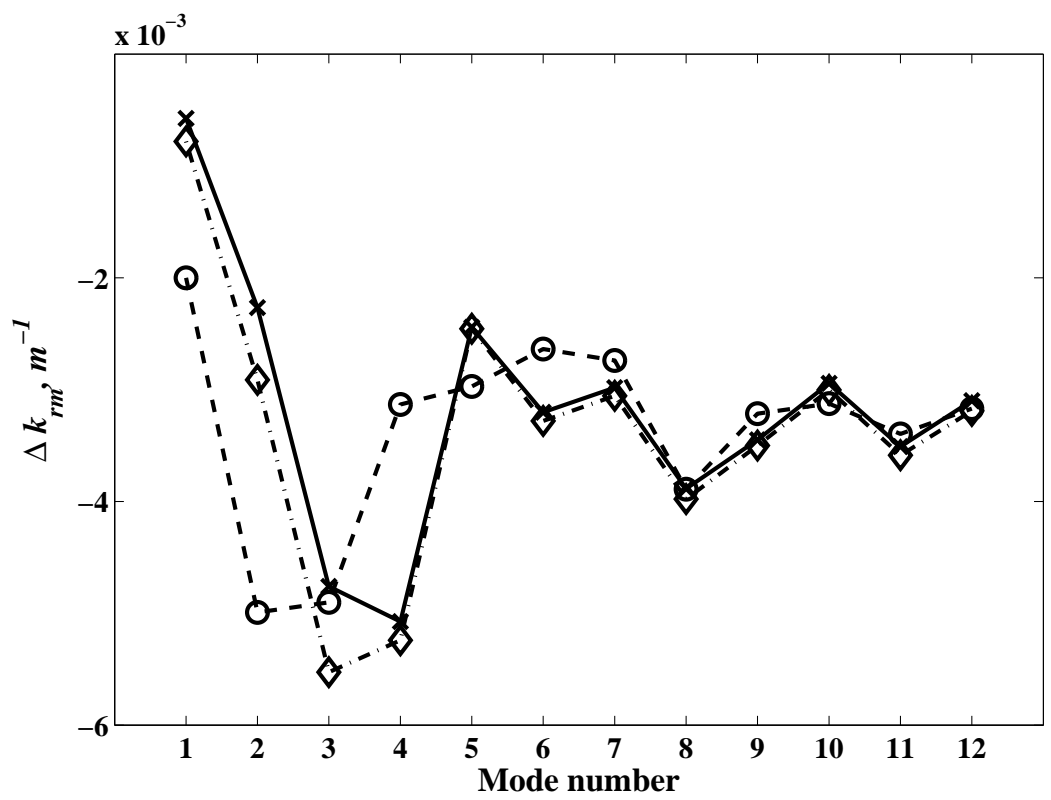

Figure 4-6: Correction to the background horizontal modal wavenumber for frequency $200 \mathrm{~Hz}$ due to the internal wave of fifteen meter amplitude, computed numerically (solid line), using perturbation method (dashed), and using a three step iterative perturbation method (dot-dashed).
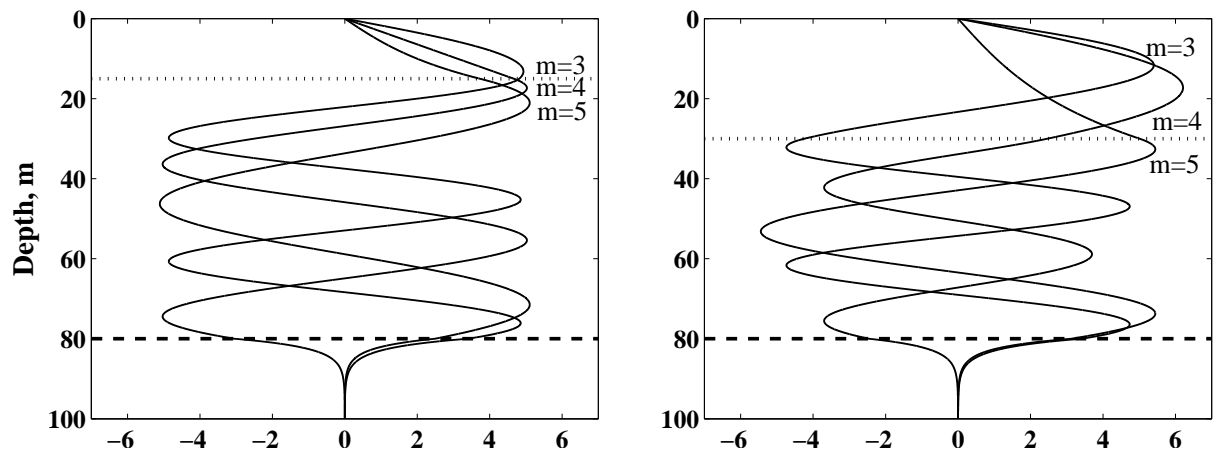

Figure 4-7: Normal modes 3-5 for background waveguide model (left) and inside a wave of fifteen meter amplitude (right). Dashed line represents the water-bottom interface; the interface between water layers (thermocline) is shown as a dotted line 


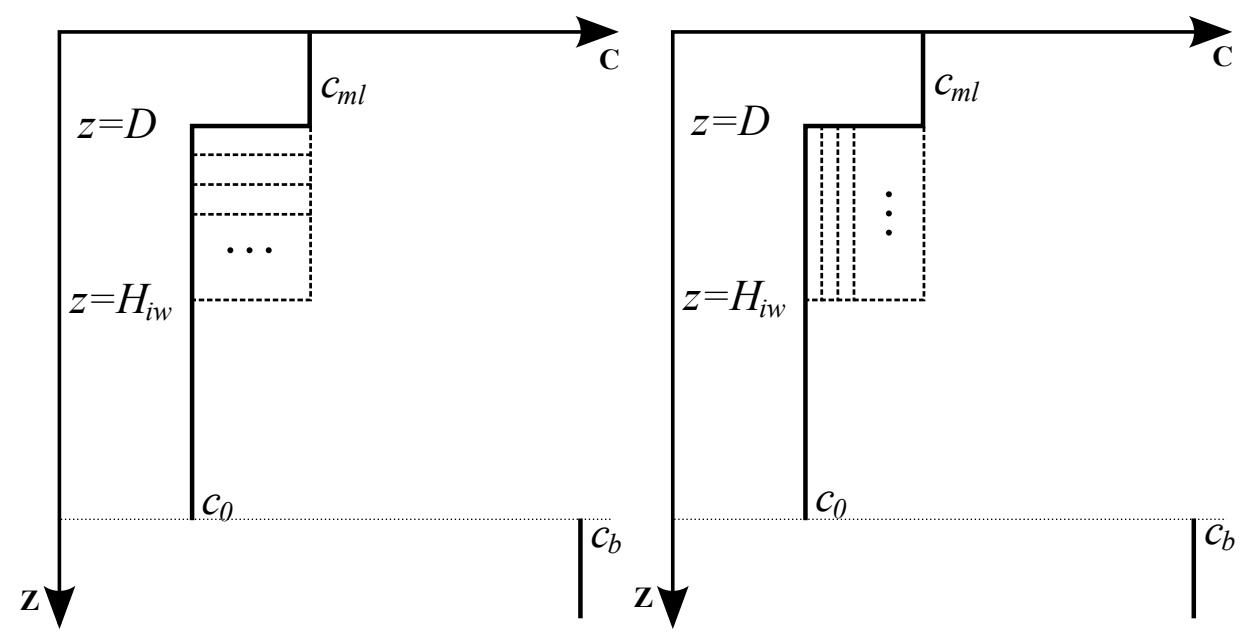

Figure 4-8: Two methods of the background water column multi-step perturbation due to internal waves: stepwise displacement of the thermocline (left); stepwise increase of sound speed (right).

- 4. This tells us that the wave amplitude of fifteen meters is too big for a single step perturbation. To account for this, multi-step perturbations can be applied by either dividing the thermocline displacement into several steps or by a stepwise increase of the sound speed from $c_{0}$ to $c_{m l}=c_{0}+\Delta c$ (Fig. 4-8). The latter method involves computation of normal mode perturbations defined by Eq. (4.22) and in general requires less steps in order to converge to a numerical solution. However, using the first method, we are able to use our closed form solution of Eq. (4.29) at each step. For doing this, we substitute $H_{i w}, k_{r m}^{1}, k_{z m}^{1}$, and $k_{z m, m l}^{1}$ at the previous step by $D, k_{r m}, k_{z m}$, and $k_{z m, m l}$ respectively at the next step. As a result, splitting the water column perturbation into several steps approaches the final modal horizontal wavenumber correction to the numerically computed values while keeping the logic of our single-step solution valid at each step. As an illustration, the dot-dashed line in Fig. 4-6 represents a five-step perturbation result with a thermocline displacement of three meters at each step. Obviously, the multi-step perturbation provides good agreement with the numerically computed wavenumber correction.

The behavior of the mode number dependence of the wavenumber correction shown in Fig. 4-6 can also be treated by examining the shape of the mode functions. Modes $3-5$ at a frequency of $200 \mathrm{~Hz}$ for the background waveguide and inside 


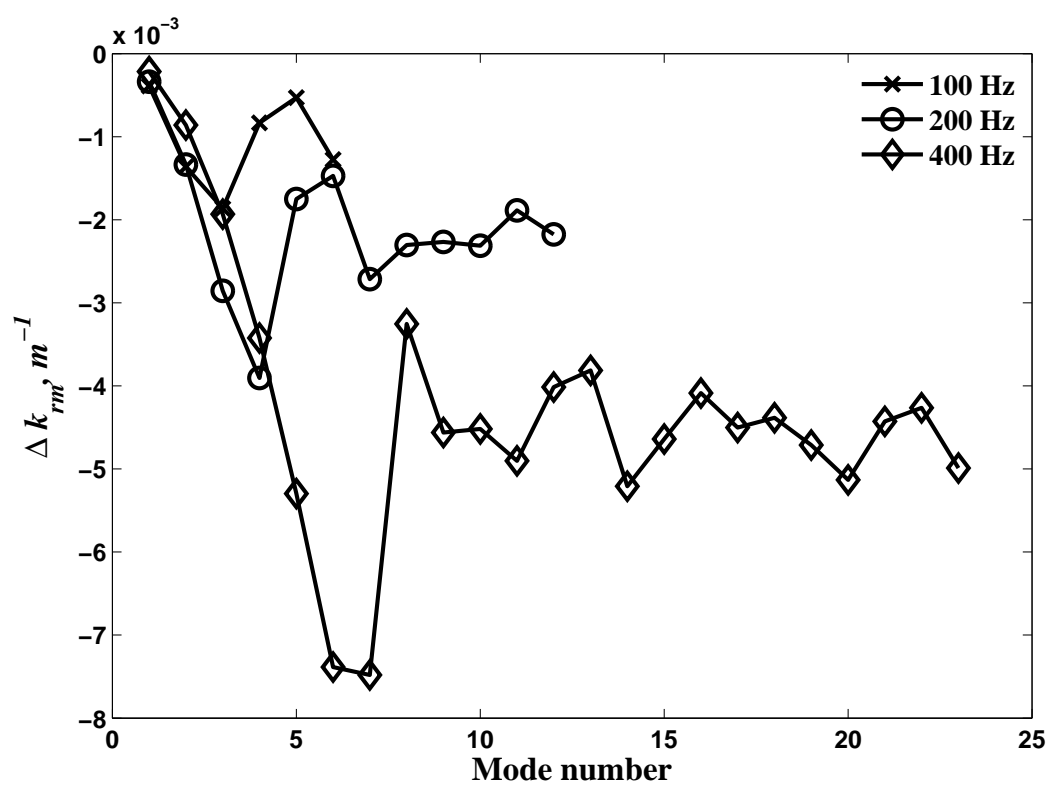

Figure 4-9: Correction to the background horizontal modal wavenumber for frequencies $100 \mathrm{~Hz}, 200 \mathrm{~Hz}$ and $400 \mathrm{~Hz}$, due to internal wave of fifteen meter amplitude.

the wave are plotted in Fig. 4-7. Dashed and dotted lines on the plots represent the water-bottom interface and the interface between water layers (the thermocline) respectively. Mode 3 has an exponentially decaying tail in the mixed layer in both the unperturbed (left panel) and perturbed (right panel) waveguides that corresponds to an imaginary vertical wavenumber inside the mixed layer, $\gamma_{m l, n}$. On the other hand, mode 5 has a turning point inside the mixed layer at both panels, implying real $\gamma_{m l, n}$. Interestingly, mode 4 has an exponential tail in the unperturbed mixed layer, and a turning point in perturbed mixed layer. Similarly to the bottom critical grazing angle, defined by Eq. (3.25), that separates propagating modes from the continuous spectrum, there is a critical grazing angle for the boundary between water layers,

$$
\theta_{c r i t, m l}=\cos ^{-1}\left(\frac{c_{0}}{c_{m l}}\right),
$$

that separates propagating modes into two groups. The first group is characterized by grazing angles smaller than $\theta_{c r i t, m l}$, and consists of lower order modes (modes 1-3 in the example above) that are perfectly trapped in between the mixed layer and the bottom half space. A small thermocline depression is "felt" by these modes as 
an effective decrease in the depth of the waveguide, resulting in an increase of the horizontal modal wavenumber contribution with mode number. The second group of propagating modes with higher mode number (modes 4-12 in the example above) has steeper than critical grazing angles, and is trapped between the surface and the bottom half space, i.e. within the whole water column. For these modes, the impact of the thermocline displacement is dominated by the number of turning points inside the mixed layer, resulting in the oscillatory behavior of the horizontal wavenumber contribution for higher mode numbers. Mode four in the example transits from the first group into the second group as the thermocline depth lowers.

Figure 4-9 shows corrections for the modal horizontal wavenumbers for the same scenario and frequencies $100 \mathrm{~Hz}, 200 \mathrm{~Hz}$, and $400 \mathrm{~Hz}$. As we see, the curves have similar shapes, with approximately equal slopes for low the order modes. However, the number of modes in each group, as well as the peak values of $\Delta k_{r m}$, increase with frequency.

\subsection{Bottom Waves}

In this section, we use our two-layer water column model to investigate the physics of the modal wavenumber perturbation due to bottom waves. In analogy to the internal waves approximation, bottom waves are modeled by a seabed elevation from $z=H_{b o t}$ to $z=H_{b o t}-\Delta H_{b o t}$ (Fig. 4-10). We desire to find an approximate expression for the horizontal modal wavenumber perturbation amplitude $\Delta k_{r m}$ due to presence of such a wave. Unfortunately, the normal mode perturbation theory that we used in Section 4.2 is not practical in this case, because it requires small relative sound speed changes $\frac{\Delta c(z)}{c(z)}$. The sound speed values in the shallow water seabed are typically at least ten percent larger than the reference sound speed in the water. In addition, density change increases plane wave impedance contrast even more, making the application of the standard first order modal perturbation theory to our problem inaccurate.

An alternative approach is proposed by considering the transcendental equation (Eq. (4.14)), and its linear perturbation due to a change in a water depth. For 


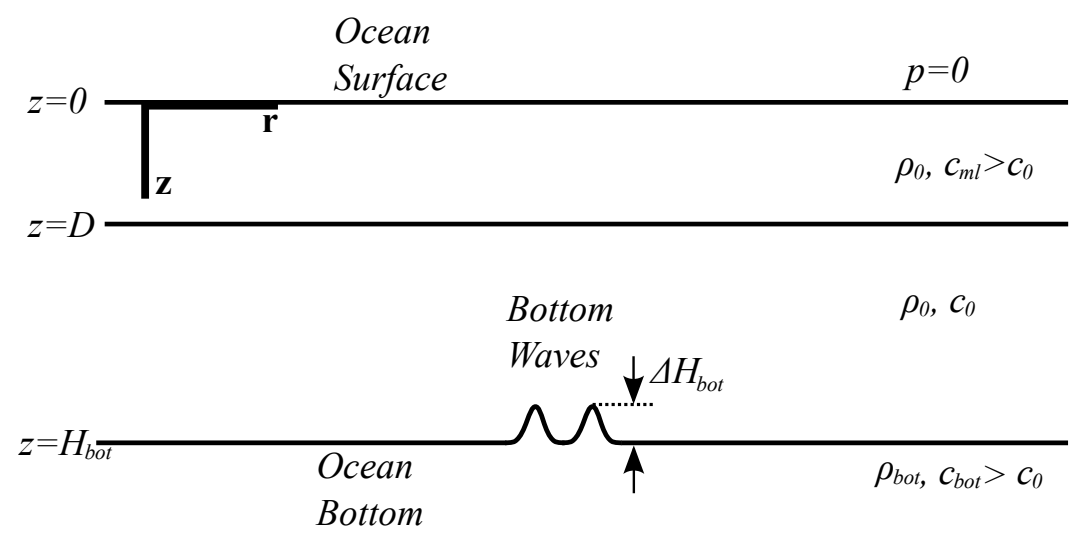

Figure 4-10: Bottom waves approximation by lifting the water-seabed interface.

shorthand, define

$$
\begin{aligned}
& P\left(k_{z m}\right)= \\
& i \frac{\left(\rho_{b o t} k_{z m}-\rho_{0} k_{z m, b o t}\right)\left(i \tan \left(k_{z m, m l} D\right) k_{z m}+k_{z m, m l}\right)-\left(\rho_{b o t} k_{z m}+\rho_{0} k_{z m, b o t}\right) e^{2 i k_{z m} D}\left(i \tan \left(k_{z m, m l} D\right) k_{z m}-k_{z m, m l}\right)}{\left(\rho_{b o t} k_{z m}-\rho_{0} k_{z m, b o t}\right)\left(i \tan \left(k_{z m, m l} D\right) k_{z m}+k_{z m, m l}\right)+\left(\rho_{b o t} k_{z m}+\rho_{0} k_{z m, b o t}\right) e^{2 i k_{z m} D\left(i \tan \left(k_{z m, m l} D\right) k_{z m}-k_{z m, m l}\right)} .}
\end{aligned}
$$

Similar to the perturbation by internal waves, we define the new horizontal modal wavenumber and its correction by Eq. (4.17). Corresponding corrections to the vertical wavenumbers for the upper mixed layer, lower layer, and bottom half space are formally written as

$$
\begin{aligned}
\Delta k_{z m} & =k_{z m}^{1}-k_{z m}, \\
\Delta k_{z m, m l} & =k_{z m, m l}^{1}-k_{z m, m l}, \\
\Delta k_{z m, b o t} & =k_{z m, b o t}^{1}-k_{z m, b o t},
\end{aligned}
$$

where the new vertical wavenumbers $k_{z m}^{1}, k_{z m, m l}^{1}$ and $k_{z m, b o t}^{1}$ are defined by Eqs. (4.18)(4.20). The transcendental equation for the modified waveguide is then

$$
\tan \left(k_{z m}^{1}\left(H_{b o t}-\Delta H_{b o t}\right)\right)=P\left(k_{z m}^{1}\right) .
$$

By equating the linear terms of a Taylor series expansion applied to both sides of Eq. 
(4.36) and neglecting the second order term $\Delta k_{z m} \Delta H$, we get

$$
\left(1+\tan ^{2}\left(k_{z m} H_{b o t}\right)\right)\left(\Delta k_{z m} H_{b o t}+k_{z m} \Delta H_{b o t}\right) \approx \frac{d P\left(k_{z m}\right)}{d k_{z m}} \Delta k_{z m}
$$

Assuming $\Delta k_{r m}<<k_{r m}$ for small $\Delta H_{b o t}$, vertical wavenumber corrections for two layers and bottom half space are related to the horizontal wavenumber correction by linearizing Eqs. (4.18)-(4.20) as

$$
\begin{aligned}
\Delta k_{z m} & \approx-\frac{k_{r m}}{k_{z m}} \Delta k_{r m}, \\
\Delta k_{z m, m l} & \approx-\frac{k_{r m}}{k_{z m, m l}} \Delta k_{r m} \\
\Delta k_{z m, b o t} & \approx-\frac{k_{r m}}{k_{z m, b o t}} \Delta k_{r m} .
\end{aligned}
$$

With Eqs. (4.37) and (4.38), we get the first order correction to the horizontal modal wavenumber

$$
\Delta k_{r m} \approx-\frac{k_{z m}^{2}}{k_{r m}} \frac{\left(1+\tan ^{2}\left(k_{z m} H_{b o t}\right)\right) \Delta H_{b o t}}{\left(1+\tan ^{2}\left(k_{z m} H_{b o t}\right)\right) H_{b o t}-\frac{d P\left(k_{z m}\right)}{d k_{z m}}} .
$$

It can be shown using simple algebra and the linearized relations of Eqs. (4.39)-(4.40) that

$$
\begin{aligned}
& \frac{d P\left(k_{z m}\right)}{d k_{z m}}= \\
& -4 i e^{2 i k_{z m} D} \frac{\rho_{0} \rho_{b o t} \frac{k_{z m, b o t}^{2}-k_{z m}^{2}}{k_{z m m}, b o t}\left(k_{z m, m l}^{2}+\tan ^{2}\left(k_{z m, m l} D\right) k_{z m}^{2}\right)+i\left(\rho_{0}^{2} k_{z m}^{2}-\rho_{b o t} k_{z m}^{2}\right) \frac{k_{z m}^{2}-k_{z m, m l}^{2}}{k_{z, m}, m l}\left(\tan \left(k_{z m, m l} D\right)-k_{z m, m l} D\right)}{\left[\left(\rho_{b o t} k_{z m}-\rho_{0} k_{z m, b o t}\right)\left(i \tan \left(k_{z m, m l} D\right) k_{z m}+k_{z m, m l}\right)+\left(\rho_{b o t} k_{z m}+\rho_{0} k_{z m, b o t}\right) e^{2 i k_{z} D} D\left(i \tan \left(k_{z m, m l} D\right) k_{z m}-k_{z m, m l}\right)\right]^{2}} .
\end{aligned}
$$

From numerical computations we have noticed that for small bottom wave amplitudes and low frequencies (typically less than $500 \mathrm{~Hz}$ ),

$$
\frac{d P}{d k_{z m}}<<\left(1+\tan ^{2}\left(k_{z m} H_{b o t}\right)\right) H_{b o t}
$$

This simplifies our solution to

$$
\Delta k_{r m} \approx-\frac{k_{z m}^{2}}{k_{r m}} \frac{\Delta H_{b o t}}{H_{b o t}}
$$




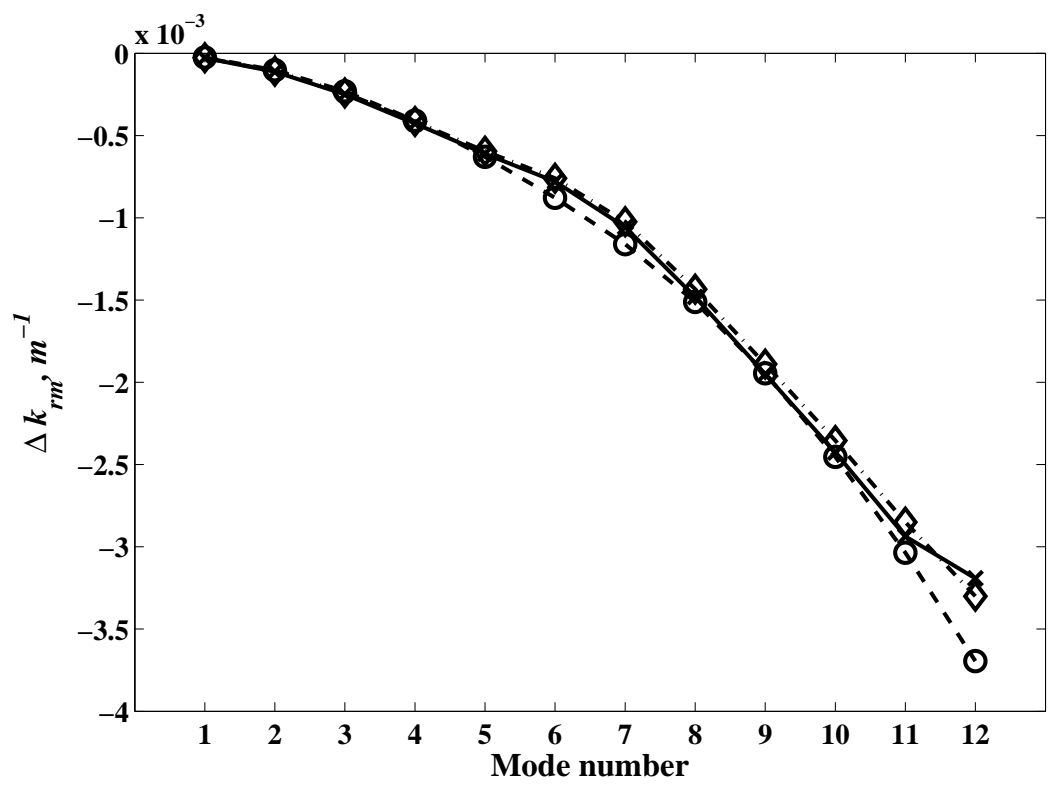

Figure 4-11: Correction to the background horizontal modal wavenumber for frequency $200 \mathrm{~Hz}$ due to presence of bottom wave of one meter amplitude, computed numerically (solid line), using linear approximation (4.41) (dot-dashed line), and using simplified linear approximation (4.44) (dashed line).

It is easy to show that Eq. (4.44) also describes the modal wavenumber correction due to seafloor elevation in an ideal waveguide with homogeneous sound speed $c_{0}$ and depth $H_{b o t}$. Thus, in shallow water the first order modal wavenumber response to bottom waves of small amplitude is governed by the equivalent response in an isovelocity waveguide with a hard bottom.

Let us return to the example of our two-layer waveguide model discussed in previous sections and consider a bottom wave of one meter amplitude. Figure 4-11 shows the numerical solution for the modal horizontal wavenumber correction (solid line) at frequency $200 \mathrm{~Hz}$, its linear approximation defined by Eq. (4.41) plotted with a dashed line, and the reduced approximation of Eq. (4.44) plotted with a dot-dashed line. As we see from the figure, although omitting $\frac{d P}{d k_{z m}}$ term provides a slight deviation of the approximate solution from the exact numerical solution for modes 6 , 7 , and 12, all three curves are in good agreement. Unlike what we have seen for internal waves, bottom waves provide a gradual monotonic increase of the horizontal wavenumber correction amplitude with mode number. 


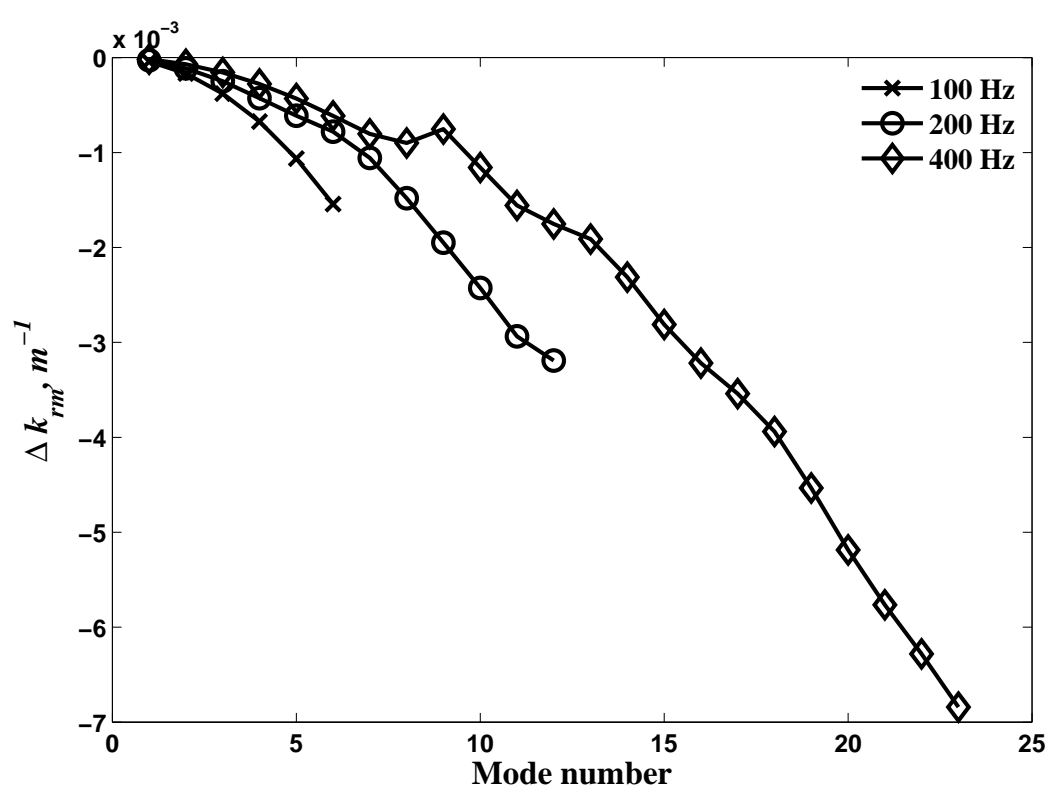

Figure 4-12: Correction to the background horizontal modal wavenumber for frequencies $100 \mathrm{~Hz}, 200 \mathrm{~Hz}$ and $400 \mathrm{~Hz}$, due to bottom wave of one meter amplitude.

Figure 4-12 shows numerically computed wavenumber corrections at frequencies $100 \mathrm{~Hz}, 200 \mathrm{~Hz}$ and $400 \mathrm{~Hz}$. As one can see, the gradually increasing trend of the wavenumber perturbation magnitude with mode number predicted by Eq. (4.44) is seen for all considered frequencies.

\subsection{Surface Waves}

Following the logic of Sections 4.2 and 4.4, we approximate ocean surface waves of amplitude $\Delta H_{\text {sur }}$ for our two-layer model as a depression (wave trough), and elevation (wave crest) of the free surface as shown in Fig. 4-13. As before, we seek the first order correction to the amplitude of the modal wavenumber perturbation caused by these waves. We note that both wave crests and wave troughs represent a displacement of the free surface, and therefore, separate expressions for reference horizontal modal wavenumber corrections should be obtained for them. In order to do that, we split the water column perturbation due to the wave crest (trough) into two steps: depression (elevation) of the thermocline by $\Delta H_{\text {sur }} / 2$ followed by the increase (decrease) of the water column depth by $\Delta H_{\text {sur }} / 2$. The top and bottom panels of Fig. 4-14 


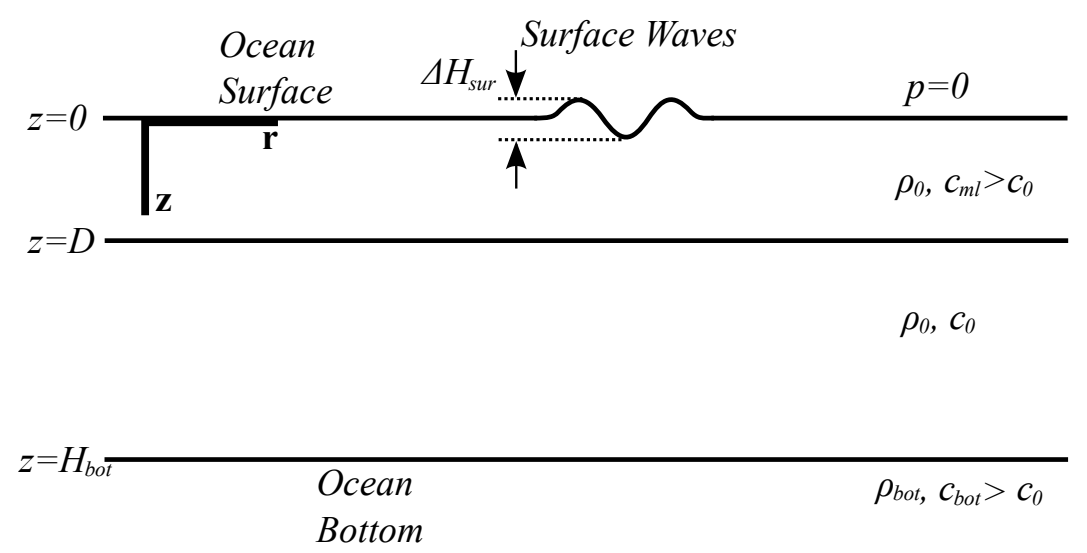

Figure 4-13: Ocean surface waves approximated by elevation and depression of the ocean surface.

schematically show a two step perturbation of the background water column due to surface wave crest and trough respectively. For the wave crest, the first step can be represented as an internal wave of amplitude $\Delta H_{\text {sur }} / 2$, and its contribution to the modal wavenumber is determined by Eq. (4.29):

$$
\Delta k_{r m, \text { step } 1}^{\text {crest }} \approx-2 \frac{\left|A_{m}\right|^{2} \omega^{2} \Delta c}{k_{r m} c_{0}^{3} \rho_{0}}\left[\frac{\Delta H_{\text {sur }}}{2}+\frac{\sin \left(k_{z m} \frac{\Delta H_{\text {sur }}}{2}\right) \cos \left(2 \tan ^{-1}\left[\frac{k_{z m, m l}}{k_{z m} \tan \left(k_{z m, m l} D\right)}\right]+k_{z m} \frac{\Delta H_{\text {sur }}}{2}\right)}{k_{z m}}\right]
$$

At the second step, we note that the impact of a bottom depression by $\Delta H_{\text {sur }} / 2$ on modal wavenumber, corrected at the first step, is exactly opposite to a bottom wave of the amplitude $\Delta H_{\text {sur }} / 2$. Therefore, by using Eq. (4.41) and substituting $\Delta H_{\text {bot }}$ by $-\Delta H_{\text {sur }} / 2$, we get the second step wavenumber correction:

$$
\Delta k_{r m, \text { step } 2}^{\text {crest }} \approx \frac{\frac{\omega^{2}}{c_{0}^{2}}-\left(k_{r m}+\Delta k_{r m, 1}^{c r e s t}\right)^{2}}{k_{r m}+\Delta k_{r m, 1}^{c r e s t}} \frac{\Delta H_{\text {sur }}}{2 H_{\text {bot }}}
$$

By neglecting second order terms $\Delta k_{r m, \text { step } 1}^{\text {crest }} \Delta H_{\text {sur }}$, this reduces to

$$
\Delta k_{r m, \text { step } 2}^{\text {crest }} \approx \frac{k_{z m}^{2}}{k_{r m}} \frac{\Delta H_{\text {sur }}}{2 H_{\text {bot }}}
$$




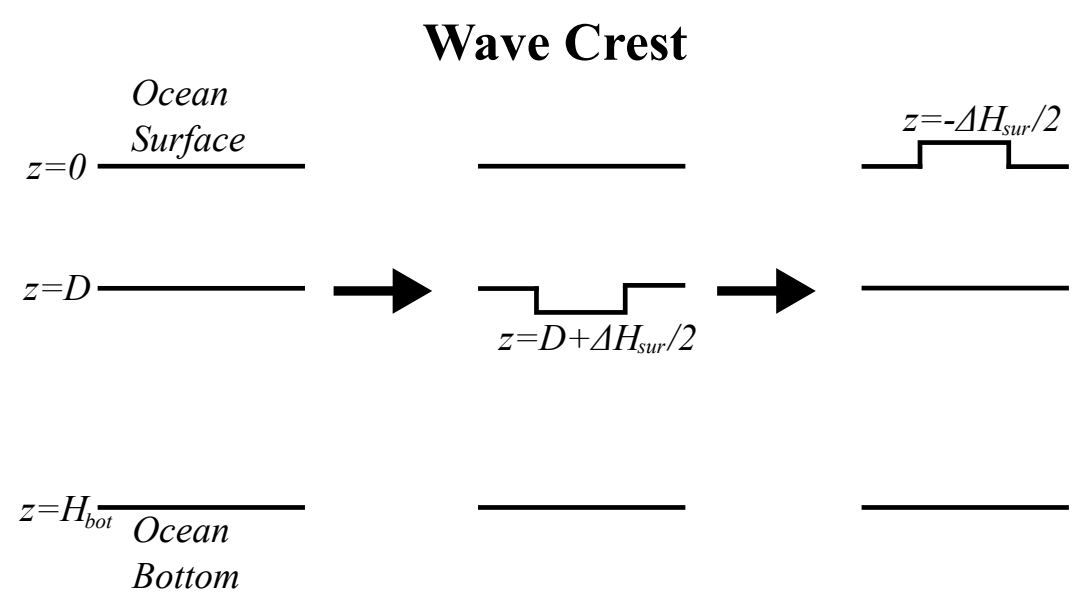

Wave Trough

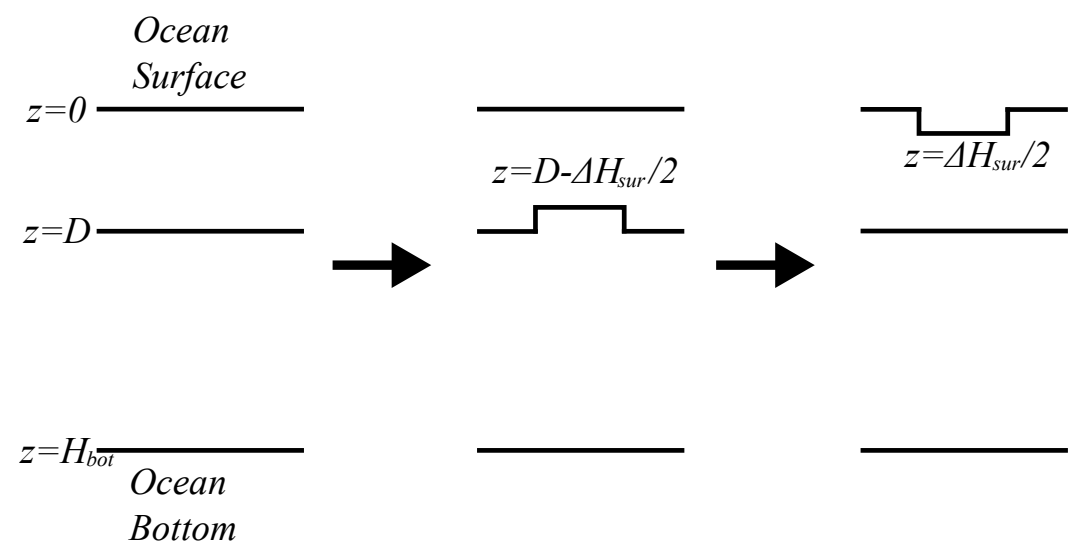

Figure 4-14: Schematic view of two steps water column perturbation due to surface wave crest (trough): thermocline depression (elevation) by $\Delta H_{\text {sur }} / 2$ followed by the water depth increase (decrease) by $\Delta H_{\text {sur }} / 2$ 
The total first order horizontal modal wavenumber contribution by the wave crest is then

$$
\begin{aligned}
\Delta k_{r m}^{\text {crest }} \approx & -2 \frac{\left|A_{m}\right|^{2} \omega^{2} \Delta c}{k_{r m} c_{0}^{3} \rho_{0}}\left[\frac{\Delta H_{\text {sur }}}{2}+\frac{\sin \left(k_{z m} \frac{\Delta H_{\text {sur }}}{2}\right) \cos \left(2 \tan ^{-1}\left[\frac{k_{z m, m l}}{k_{z m} \tan \left(k_{z m, m l} D\right)}\right]+k_{z m} \frac{\Delta H_{\text {sur }}}{2}\right)}{k_{z m}}\right] \\
& +\frac{k_{z m}^{2}}{k_{r m}} \frac{\Delta H_{\text {sur }}}{2 H_{\text {bot }}} .
\end{aligned}
$$

By analogy, applying two steps with the opposite perturbations of the background environment for small $\Delta H_{\text {sur }}$, the horizontal modal wavenumber correction for wave trough is equal to that for a wave crest, but opposite in sign:

$$
\Delta k_{r m}^{\text {trough }} \approx-\Delta k_{r m}^{\text {crest }}
$$

The amplitude of the total modal wavenumber variation in the presence of the surface waves of amplitude $\Delta H_{\text {sur }}$ is the difference between the wavenumbers for the wave trough and the wave crest:

$$
\begin{gathered}
\Delta k_{r m}=\Delta k_{r m}^{\text {trough }}-\Delta k_{r m}^{\text {crest }} \approx \\
4 \frac{\left|A_{m}\right|^{2} \omega^{2} \Delta c}{k_{r m} c_{0}^{3} \rho_{0}}\left[\frac{\Delta H_{\text {sur }}}{2}+\frac{\sin \left(k_{z m} \frac{\Delta H_{\text {sur }}}{2}\right) \cos \left(2 \tan ^{-1}\left[\frac{k_{z m, m l}}{k_{z m} \tan \left(k_{z m, m l} D\right)}\right]+k_{z m} \frac{\Delta H_{s u r}}{2}\right)}{k_{z m}}\right]-\frac{k_{z m}^{2}}{k_{r m}} \frac{\Delta H_{\text {sur }}}{H_{\text {bot }}} .
\end{gathered}
$$

The first term in Eq. (4.50) is positive, because it corresponds to an effective elevation of the thermocline by the value $\Delta H_{\text {sur }}$. Similarly to depression internal waves, it increases in amplitude up to a certain mode number, following by oscillations (see discussions in Section 4.2 for details). The second term has a negative value, and its wavenumber contribution is equal to that of bottom waves of amplitude $\Delta H_{\text {sur }}$.

Let us return to the example of the waveguide discussed in the previous sections with surface waves of one-meter amplitude $\left(\Delta H_{\text {sur }}=1 \mathrm{~m}\right)$. Figure 4 - 15 shows a good agreement between the numerical solution for the total horizontal modal wavenumber variation (solid curve) and its first order perturbation (dashed curve) defined by Eq. (4.50) at frequency $200 \mathrm{~Hz}$. Some slight deviation between the exact and approximate solutions for modes 5, 6, and 12, also noted for the perturbation due to bottom 
waves in the same waveguide, is caused by using the reduced form of the wavenumber perturbation (Eq. (4.44)) by bottom waves (see Section 4.3 for details). Interestingly, unlike the case of internal waves or bottom waves, the curves have zero initial slope, i.e. for low order modes, the two terms in Eq. (4.50) have opposite values and cancel each other. Physically, this implies that these modes have vertical grazing angles less than the mixed layer critical angle defined by Eq. (4.31). They are trapped between the mixed layer and the bottom half space, and therefore, small perturbations of the ocean surface have no impact on the horizontal wavenumbers of these modes. Higher order modes have turning points in the mixed layer, and are affected by mutual effect of the effective thermocline displacement and the change in water depth. As a result, we see an increase in wavenumber perturbation amplitude with fluctuating slope for mode numbers higher than 4 .

Figure 4-16 shows the numerical solution for the modal wavenumber variation at frequencies $100 \mathrm{~Hz}, 200 \mathrm{~Hz}$ and $400 \mathrm{~Hz}$. It is noted that at higher frequencies, there are more modes unaffected by the presence of surface waves. This follows from the closer spacing of the vertical modal wavenumbers and the corresponding modal vertical grazing angles. Fluctuation of the curve slope caused by effective displacement of the thermocline is also more noticeable for higher frequency. Thus, with increasing frequency, the impact of the effective thermocline displacement (the first step in the water column perturbation due to surface waves) on horizontal modal wavenumber becomes stronger. In fact, at frequency $100 \mathrm{~Hz}$, there is almost no difference between the wavenumber perturbation due to surface waves and due to bottom waves of the same amplitude (see $100 \mathrm{~Hz}$ curve in Fig. 4-12 for comparison). 


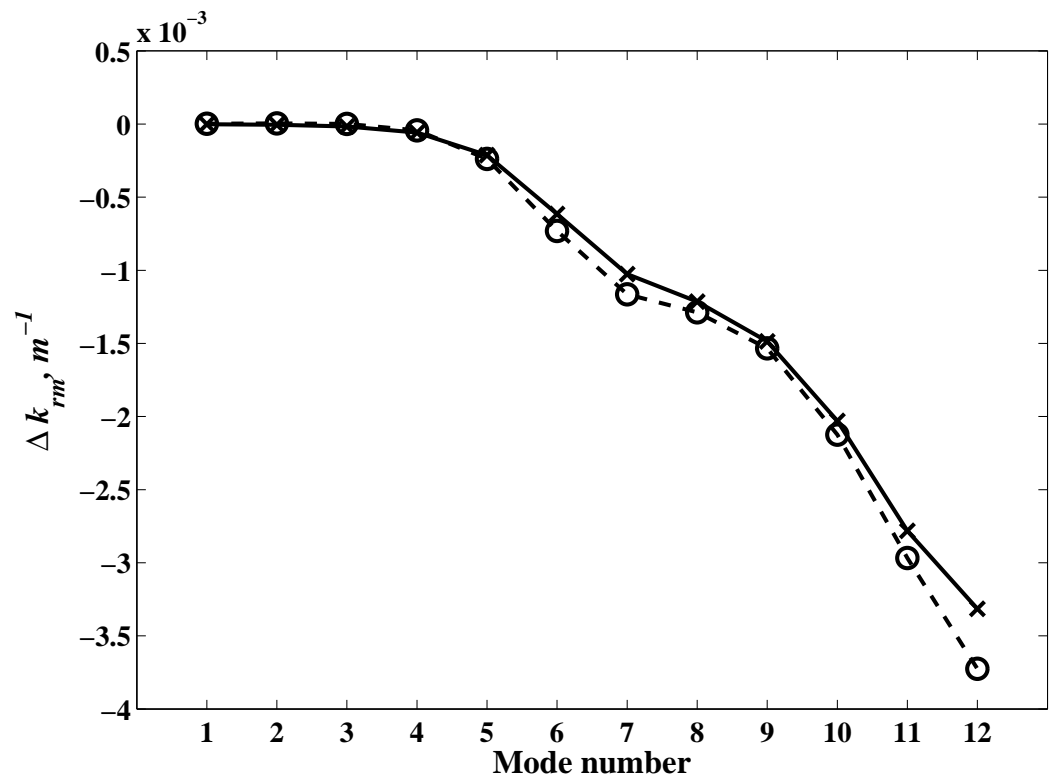

Figure 4-15: Modal wavenumber variation amplitude for frequency $200 \mathrm{~Hz}$ due to the presence of surface waves with one meter amplitude. The numerical solution is plotted with a solid line; the dashed line represents the first order approximation.

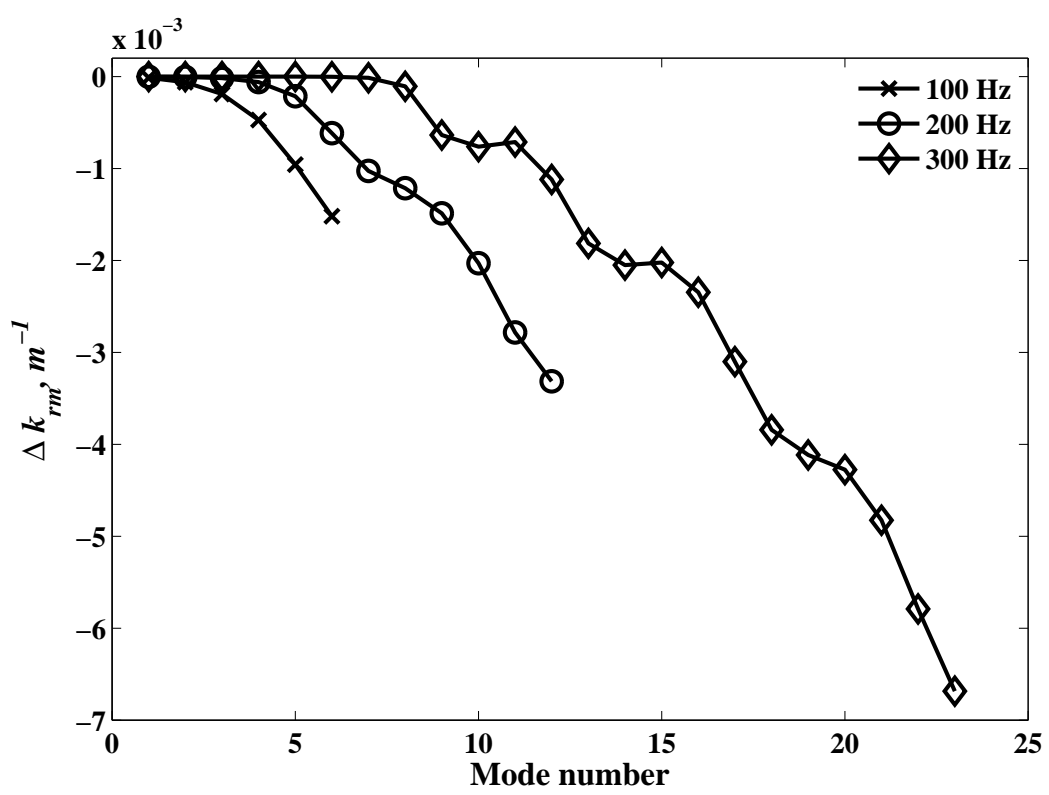

Figure 4-16: Numerical solution for the modal wavenumber variation amplitude at frequencies $100 \mathrm{~Hz}, 200 \mathrm{~Hz}$ and $400 \mathrm{~Hz}$, due to presence of surface waves with one meter amplitude. 


\section{Chapter 5}

\section{Acoustic Propagation in Presence of Infinitely Long Parallel Waves}

In the real ocean, each of the types of waves we consider is characterized by finite crest lengths and horizontal curvature of the wave fronts. In order to understand the physics of low frequency acoustic interaction with the realistic wave fields, it is essential to first determine the mechanisms that govern acoustic propagation in presence of idealistic waves with infinitely long parallel crests. In this section, we will examine three-dimensional acoustic effects that occur in presence of ocean internal waves, bottom sediment waves and surface waves in shallow water. As before, we will use the normal mode approach for representation of the pressure field and examine the effects of normal mode coupling, refraction, ducting and antiducting in presence of one or several parallel waves of any of the three types.

\subsection{Horizontal Modal Critical Angle}

Consider the example of a horizontally stratified shallow water waveguide with identical waves that have infinitely long crests parallel to the $x$-axis (Fig. 5-1). Define unperturbed modal wavenumber (outside of the waves) by $k_{r n}^{0}$ and its most perturbed value by $k_{r n}^{1}$ (along the wave crest), such that modal horizontal wavenumber at arbi- 
trary point in horizontal plane satisfies

$$
k_{r n}^{0} \geq k_{r n}(x, y) \geq k_{r n}^{1}
$$

Note that perturbed modal horizontal wavenumber is less than its unperturbed value, because we define perturbation by either bottom elevation (bottom wave), thermocline depression (internal wave), or surface depression (surface wave). Also note that we use index $n$ in this chapter to denote the number of the initial mode. We don't specify the wave type for now, but we assume that the width of the wave is wide enough and changes of the horizontal modal wavenumber across the wave are small enough so that the adiabatic approximation is valid. For such environments, it is convenient to use the "Vertical Modes - Horizontal Rays" formalism of Weinberg and Burridge [36] for studying the acoustic pressure field. In this approach, each normal mode propagates along a horizontal ray defined by the eikonal equation

$$
\mathbf{s}_{\mathbf{n}}=\hat{\mathbf{s}}_{\mathbf{n}} k_{r n}(x, y)
$$

where

$$
\frac{d \mathbf{s}_{\mathbf{n}}}{d s_{n}}=\nabla_{H} k_{r n}(x, y)
$$

$\hat{\mathbf{s}}_{\mathbf{n}}(x, y)$ is the unit vector in the local ray direction of mode $n, d s_{n}$ is the incremental length along the ray path, and $\nabla_{H}$ is the two-dimensional gradient.

Let us put an acoustic source outside of the wave and define the horizontal modal grazing angle $\chi_{n}$ (not to be confused with the vertical grazing angle in Chapter 3) as the angle between the direction of the modal ray and the direction of the wave crest (Fig. 5-1). Note that in this geometry, the horizontal modal wavenumber is a function of the $y$-coordinate only. By the method of characteristics, Eq. (5.2) reduces to

$$
\frac{d}{d s}\left(k_{r n} \frac{d y}{d s}\right)=\frac{d k_{r n}}{d y} .
$$




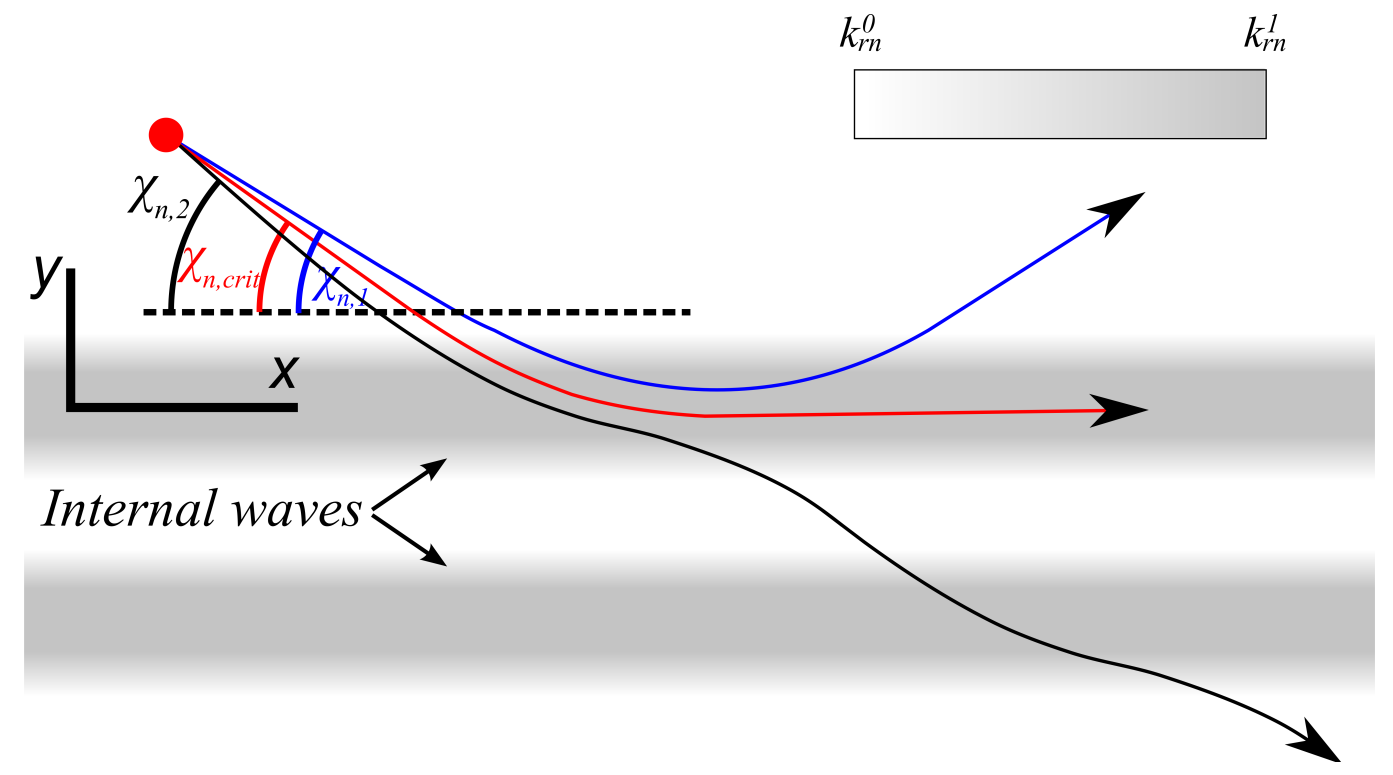

Figure 5-1: Top view of the infinitely long waves (for example, internal waves) with crests or troughs parallel to the $x$-axis. The horizontal wavenumber of mode $n$ is schematically shown in gray shading. The white unshaded region corresponds to the background unperturbed waveguide with wavenumber $k_{r n}^{0}$; the wave crest is characterized by the perturbed value $k_{r n}^{1}$. The horizontal grazing angle of a modal ray is defined as the angle between the direction of the mode $n$ ray and the direction of the wave crest. Modal rays incident from the source are divided into two groups. Rays from the first group are characterized by smaller than critical horizontal grazing angles and are refracted by the first wave back into the half space of positive $y$-values. The second group of rays have steeper than critical horizontal grazing angles. These rays penetrate through the waves and propagate into the half space of negative $y$-values. 
Consider the ray of mode $n$ launched with grazing angle $\chi_{n}$. The directional vector $\hat{\mathbf{s}}_{\mathbf{n}}$ then has initial coordinates

$$
\hat{\mathbf{s}}_{\mathbf{n}}=\left(\cos \left(\chi_{\mathbf{n}}\right), \sin \left(\chi_{\mathbf{n}}\right)\right) .
$$

As the ray propagates through the wave, its direction changes according to Eq. (5.3). There exists a critical horizontal grazing angle,

$$
\chi_{c r i t, n}=\cos ^{-1}\left(\frac{k_{r n}^{1}}{k_{r n}^{0}}\right),
$$

that separates the rays of mode $n$ into two groups. Rays with smaller than critical launched grazing angles are turned by the leading wave back into the half space of positive $y$-values (blue ray in Fig. 5-1). The second group of rays with steeper grazing angles do not have turning points and penetrate through the waves into the half space of negative $y$-values (black ray in Fig. 5-1).

The horizontal critical angle is a very important parameter of the physical mechanisms that govern three-dimensional normal mode horizontal refraction due to shallow water waves. Before proceeding to the latter, we return to our waveguide model. For consistency, we use the parameters of the unperturbed waveguide and wave amplitudes from the previous chapter $\left(c_{0}=1500 \mathrm{~m} / \mathrm{s}, c_{m l}=1530 \mathrm{~m} / \mathrm{s}, c_{\text {bot }}=1800 \mathrm{~m} / \mathrm{s}, \rho_{0}\right.$ $=1000 \mathrm{~kg} / \mathrm{m}^{3}, \rho_{b o t}=2000 \mathrm{~kg} / \mathrm{m}^{3}, H_{b o t}=80 \mathrm{~m}, D=15 \mathrm{~m}, \Delta H_{\text {iw }}=15 \mathrm{~m}, \Delta H_{b o t}=1$ $\mathrm{m}$ and $\Delta H_{\text {sur }}=1 \mathrm{~m}$ ). Using Eq. (5.5) and the wavenumber corrections found in the previous chapter, the horizontal critical angles are shown in Fig. 5-2 for frequencies $100 \mathrm{~Hz}, 200 \mathrm{~Hz}$ and $400 \mathrm{~Hz}$. As one can see from this figure, the critical angle has the same signature of mode number dependence as the modal wavenumber corrections (see Figs. 4-9, 4-12, and 4-16). In particular, the highest value of the horizontal critical angle for the internal wave corresponds to the mid-number normal modes, after which it oscillates with decreasing amplitude. On the other hand, horizontal critical angle for the other two types of waves show monotonic increase with the mode number. One also notes that unlike the case of wavenumber corrections, the greatest value of the critical angle is relatively constant for the considered frequency range. 

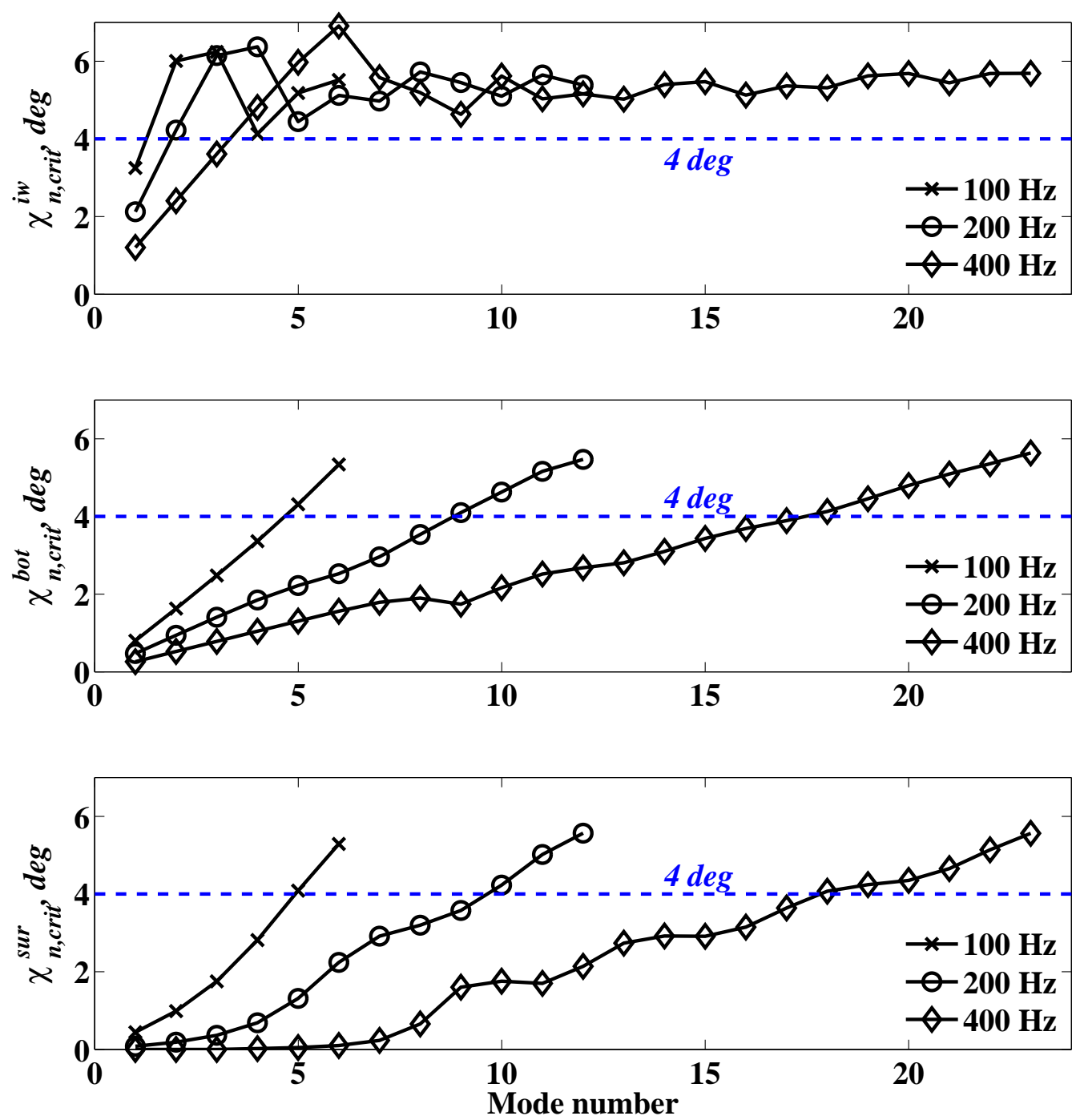

Figure 5-2: Horizontal critical angles associated with internal waves of fifteen meter amplitude (upper panel), bottom wave of one meter amplitude (middle panel) and surface wave of one meter amplitude (lower panel). Parameters of the waveguide are: $c_{0}=1500 \mathrm{~m} / \mathrm{s}, c_{m l}=1530 \mathrm{~m} / \mathrm{s}, c_{b o t}=1800 \mathrm{~m} / \mathrm{s}, \rho_{0}=1000 \mathrm{~kg} / \mathrm{m}^{3}, \rho_{\text {bot }}=2000$ $\mathrm{kg} / \mathrm{m}^{3}, H_{b o t}=80 \mathrm{~m}, D=15 \mathrm{~m}$. Wave amplitudes are: $\Delta H_{i w}=15 \mathrm{~m}, \Delta H_{b o t}=1$ $\mathrm{m}$ and $\Delta H_{\text {sur }}=1 \mathrm{~m}$. Blue dashed line shows the observed 4 deg fluctuation of the angle of arrival due to presence of internal waves (see Chapter 2). 
For examples shown here, the maximum critical angle for all waves with specified amplitudes is approximately 5.5 degrees. We refer the reader's attention to the value of $4 \mathrm{deg}$ of the observed amplitude of the angle of arrival fluctuations marked as a blue dashed line(Chapter 2). Although larger amplitude internal waves were observed during SW06 experiment, one can see that even for fifteen meter amplitude internal waves most of the modes have approximately $4 \mathrm{deg}$ or greater critical angles. The lower two panels (for the other two types of waves) preliminarily indicate that only high order modes are very refractive for the wave amplitudes provided.

\subsection{Sharp Interface Approximation}

In shallow water, significant horizontal changes of the water column and seabed properties can often happen over relatively short distances compared to the low frequency acoustic wavelength, and the adiabatic approximation becomes no longer valid. Nonlinear internal waves $[4,38]$, giant bottom waves [23], or large surface swell waves [27] are good examples for this. For the case of internal waves, low frequency acoustic propagation has been traditionally divided into four regimes [39] depending on the horizontal grazing angle. Figure 5-3 schematically shows internal waves with infinitely long parallel crests and the corresponding horizontal grazing angle sectors. For steep horizontal grazing angles, water column properties change rapidly along the direction of the acoustic track, and mode coupling (MC) is expected to be strong. As the grazing angle decreases, mode coupling weakens and acoustic propagation becomes nearly adiabatic (AD) with horizontal refraction (HR) and focusing (HF) dominating at the propagation directions nearly parallel to the IW crests.

The division into sectors shown in Fig. 5-3 is purely qualitative, since the strength of mode coupling changes gradually with the grazing angle and depends upon the waveguide parameters and the acoustic frequency. It is our goal in this chapter to explore the effects of acoustic propagation in the presence of one or more parallel waves and determine the actual importance of mode coupling or the sufficiency of the adiabatic approximation for various grazing angles. To do this, we will introduce 


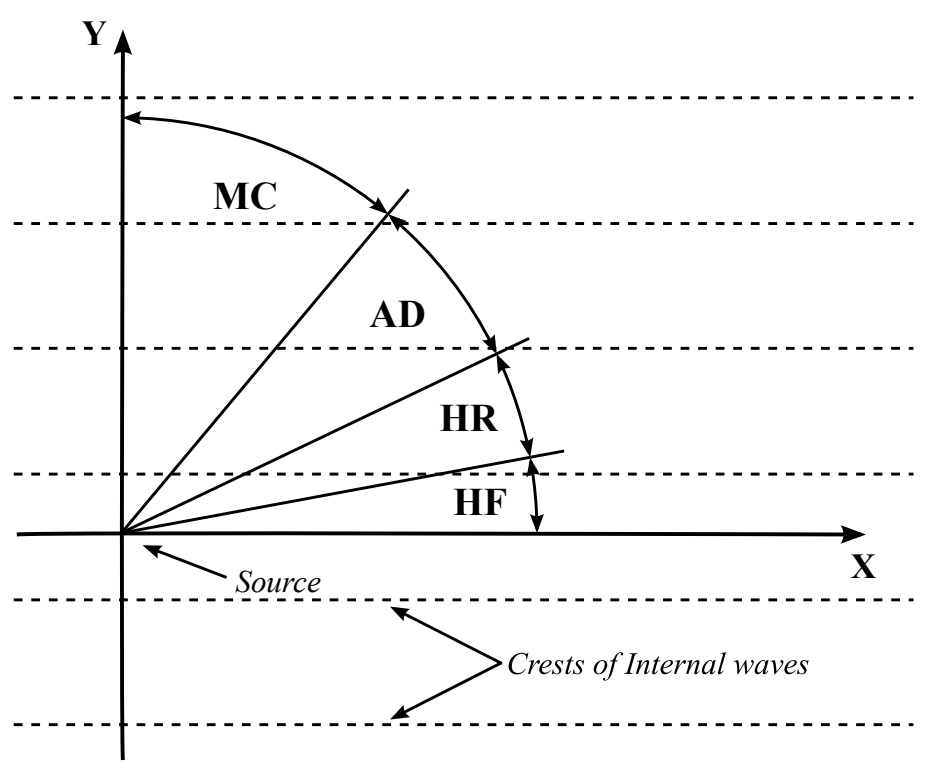

Figure 5-3: Schematic diagram showing traditional division of acoustic propagation into four sectors of grazing angles, having different propagation regimes: strong mode coupling $(\mathrm{MC})$, nearly adiabatic propagation $(\mathrm{AD})$, horizontal refraction $(\mathrm{HR})$, and horizontal focusing (HF).

an idealized wave model. In this model, we replace the smooth change in the water column properties due to a single infinitely long wave, as considered in the previous section, by a "pair of steps" so that the domain is divided into three range independent regions $\mathrm{A}, \mathrm{B}$ and $\mathrm{C}$ by infinitely long vertical interfaces parallel to the $x$-axis (Fig. 5-4). Regions A and B have an identical background water column, and region C has width $W$ and the water column properties of the wave crest. In this waveguide water column, the properties change sharply at the vertical interfaces dividing regions A, B, and C. This is called the sharp interface approximation (SIA), and the waves with corresponding shapes are called SIA waves. Figure 5-5 illustrates SIA internal, bottom and surface waves in the example of our two-layer waveguide model. SIA waves have been previously used for describing the physics of two-dimensional mode coupled propagation through nonlinear internal waves at normal incidence [4, 40]. In three-dimensional acoustics, the SIA was used under the adiabatic approximation by Lin et. al. [18] to describe the effects of horizontal acoustic ducting in between internal waves and by McMahon for theoretical studies of Horizontal Lloyds Mirror effect [41]. In the following sections, we will consider three-dimensional mode coupling 


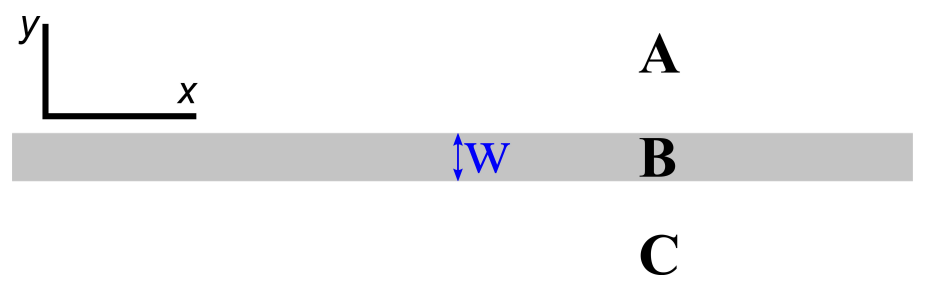

Figure 5-4: Idealized wave constructed by the two sharp vertical interfaces separated by distance $W$.



Figure 5-5: Schematic diagram showing sharp interface approximation (SIA) of internal waves, bottom waves and surface waves in the two-layer waveguide model.

in presence of infinitely long SIA waves.

The SIA has an advantage of its simplicity as it replaces the problem of continuous modal refraction and coupling with discrete modal refraction and coupling at several vertical interfaces. As we will see in the next section, it allows us to incorporate the geometric approximation for low frequency coupled mode horizontal propagation. Note that the SIA wave is characterized by abrupt changes of the modal characteristics at all grazing angles. Therefore, the strength of mode coupling effects caused by SIA waves is assumed to be overestimated in comparison to the latter in presence of realistic waves having smooth shapes. We will use SIA waves approach for semi-quantitative studying the effects of acoustic reflection, ducting, tunneling and coupling in the presence of one or several infinitely long parallel waves. The quantitative importance of these effects in presence of waves that have smooth canonical shapes will be evaluated numerically in the Section 5.6. 


\subsection{Coupled Reflection and Transmission of Nor- mal Modes at a Sharp Interface}

We begin with the fundamental problem of coupled normal mode reflection from and transmission through a single sharp interface. This scenario corresponds to infinite width of the region $\mathrm{B}$ inside the SIA wave $(W=\infty)$ in Fig. $5-4$. Region $\mathrm{A}$ is associated with the unperturbed horizontal modal wavenumbers $k_{r n}^{0}$ and the normal mode set $\Psi_{n}^{0}(z)$, and region B has values $k_{r n}^{1}$ and $\Psi_{n}^{1}(z)$ perturbed by the wave crest respectively. We wish to get the full mode coupled solution for a point source located in the region A. To do this, we first consider mode coupled reflection and transmission for individual plane waves, and then perform a plane wave decomposition of the incident point source field.

\subsubsection{Plane wave mode coupling}

When studying the effects of mode coupling, it is a standard practice $[42,4]$ to consider incident field due to each of excited normal modes separately. We let the acoustic signal be a single mode $n$ plane wave of unit amplitude at angular frequency $\omega$, and be incident upon a sharp interface from region A with grazing angle $\chi_{n}$ (Fig. 5-6). The incident pressure field can be written as

$$
p_{I}=\Psi_{n}^{0}(z) e^{i k_{r n}^{0}\left(x \cos \chi_{n}-y \sin \chi_{n}\right)}
$$

In order to match the continuity of the acoustic pressure and the normal component of particle velocity at the sharp interface,

$$
\begin{gathered}
p_{I}(x, y=0, z)+p_{R}(x, y=0, z)=p_{T}(x, y=0, z), \\
\frac{1}{\rho^{0}(z)}\left(\left.\frac{\partial p_{I}}{\partial y}\right|_{y=0}+\left.\frac{\partial p_{R}}{\partial y}\right|_{y=0}\right)=\left.\frac{1}{\rho^{1}(z)} \frac{\partial p_{T}}{\partial y}\right|_{y=0},
\end{gathered}
$$

where $\rho^{0}(z)$ and $\rho^{1}(z)$ are the density profiles in the regions $\mathrm{A}$ and $\mathrm{B}$ respectively. One must also allow reflected and transmitted fields, $p_{R}$ and $p_{T}$, to comprise all the 


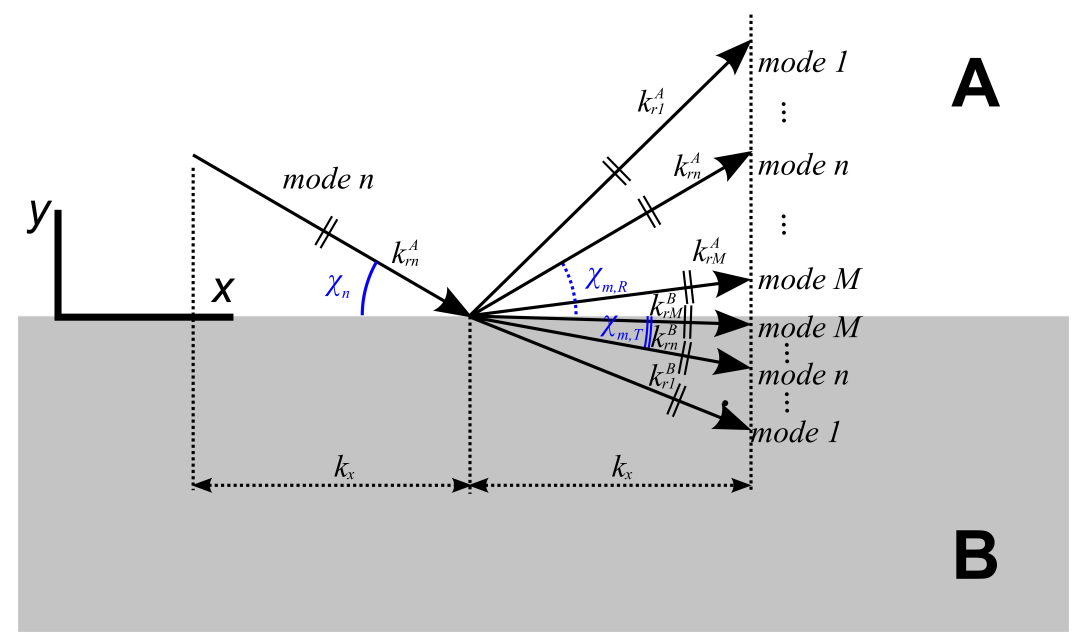

Figure 5-6: Plane wave mode coupling at the sharp interface. Incident pressure field consists of the single mode $n$ of unit amplitude with grazing angle $\chi_{n}$. Along the interface, it couples into all propagating modes $1 . . M$ of both reflected and transmitted fields.

propagating modes, i.e.

$$
\begin{aligned}
& p_{R}=\sum_{m=1}^{M} R_{n m}\left(\chi_{n}\right) \Psi_{m}^{0}(z) e^{i k_{r m}^{0}\left(x \cos \chi_{m, R}+y \sin \chi_{m, R}\right)}, \\
& p_{T}=\sum_{m=1}^{M} T_{n m}\left(\chi_{n}\right) \Psi_{m}^{1}(z) e^{i k_{r m}^{1}\left(x \cos \chi_{m, T}-y \sin \chi_{m, T}\right)},
\end{aligned}
$$

where $R_{n m}$ and $T_{n m}$ are the coefficients of the plane wave reflection and transmission of incident mode $n$ into mode $m$ and $M$ is the number of propagating modes. We made an assumption here of small waveguide perturbations at the interface, and therefore neglected associated continuous part of the spectrum. The case of modal energy leakage into the continuous part of the spectrum for surface waves will be discussed in Chapter 7.

The boundary conditions given by Eq. (5.7) provide continuity of the $x$-component of the horizontal wavenumber (Snell's law) of both the reflected and transmitted modes,

$$
k_{x} \equiv k_{r n}^{0} \cos \chi_{n}=k_{r m}^{0} \cos \chi_{m, R}=k_{r m}^{1} \cos \chi_{m, T}, \quad m=1 . . M,
$$

where $\chi_{m, R}$ and $\chi_{m, T}$ are the grazing angles of the reflected and transmitted coupled 
mode $m$. They also provide linear relations for the coupled reflection and transmission coefficients,

$$
\begin{aligned}
\delta_{n m}+R_{n m}\left(\chi_{n}\right) & =\sum_{k=1}^{M} T_{n k}\left(\chi_{n}\right) \int \frac{\Psi_{k}^{1}(z) \Psi_{m}^{0}(z)}{\rho^{0}(z)} d z, m=1 . . M, \\
\delta_{n m} \tan \chi_{n}-R_{n m}\left(\chi_{n}\right) \tan \chi_{m, R} & =\sum_{k=1}^{M} T_{n k}\left(\chi_{n}\right) \tan \chi_{k, T} \int \frac{\Psi_{k}^{1}(z) \Psi_{m}^{0}(z)}{\rho^{0}(z)} d z, m=1 . . M,
\end{aligned}
$$

where $\delta_{n m}$ is the Kronecker symbol.

As an example, Fig. 5-7 shows dependence of the absolute values of $R_{n m}$ (top panel) and $T_{n m}$ (bottom panel) on grazing angle $\chi_{n}$ for incident mode three $(n=3)$, frequency $100 \mathrm{~Hz}$, three-layer water column example from the previous chapter $\left(c_{0}=\right.$ $1500 \mathrm{~m} / \mathrm{s}, c_{m l}=1530 \mathrm{~m} / \mathrm{s}, c_{b o t}=1800 \mathrm{~m} / \mathrm{s}, \rho_{0}=1000 \mathrm{~kg} / \mathrm{m}^{3}, \rho_{b o t}=2000 \mathrm{~kg} / \mathrm{m}^{3}$, $H_{b o t}=80 \mathrm{~m}$ and $D=15 \mathrm{~m}$ ) and the waveguide perturbation due to a large twenty five meter amplitude internal wave. In this example, the horizontal critical angle of incident mode three is 6.4 degrees. As one would expect, the reflection coefficient $R_{33}$ rises rapidly as the grazing angle of mode three approaches its critical value. However, the reflection of mode three is not perfect at shallower than critical angles (note red curve below unity in the upper panel for $\chi_{3}<6.4 \mathrm{deg}$ ). At these grazing angles for incident mode three, the lower order modes (one and two) have real valued $y$-components of the horizontal wavenumber defined as

$$
k_{y m}^{0(1)} \equiv \begin{cases}\sqrt{\left(k_{r m}^{0(1)}\right)^{2}-k_{x}^{2}}, & \left|k_{x}\right| \leq k_{r m}^{0(1)}, \\ i \sqrt{k_{x}^{2}-\left(k_{r m}^{0(1)}\right)^{2}}, & \left|k_{x}\right| \geq k_{r m}^{0(1)}\end{cases}
$$

in both half spaces A and B. Therefore, even at subcritical grazing angles for mode three, energy leakage occurs by means of mode coupling. In contrast, higher order modes are evanescent at these angles, and their amplitudes decay exponentially with distance from the sharp interface. As the grazing angle of incident mode three increases, higher order modes become propagating one by one in both regions $\mathrm{A}$ and $\mathrm{B}$, and energy coupled into these modes propagates away from the interface (note the spikes in $R_{3 m}$ and $T_{3 m}$ for $m=4,5,6,7$ at grazing angles 14, 22, 28 and 25 degrees). 

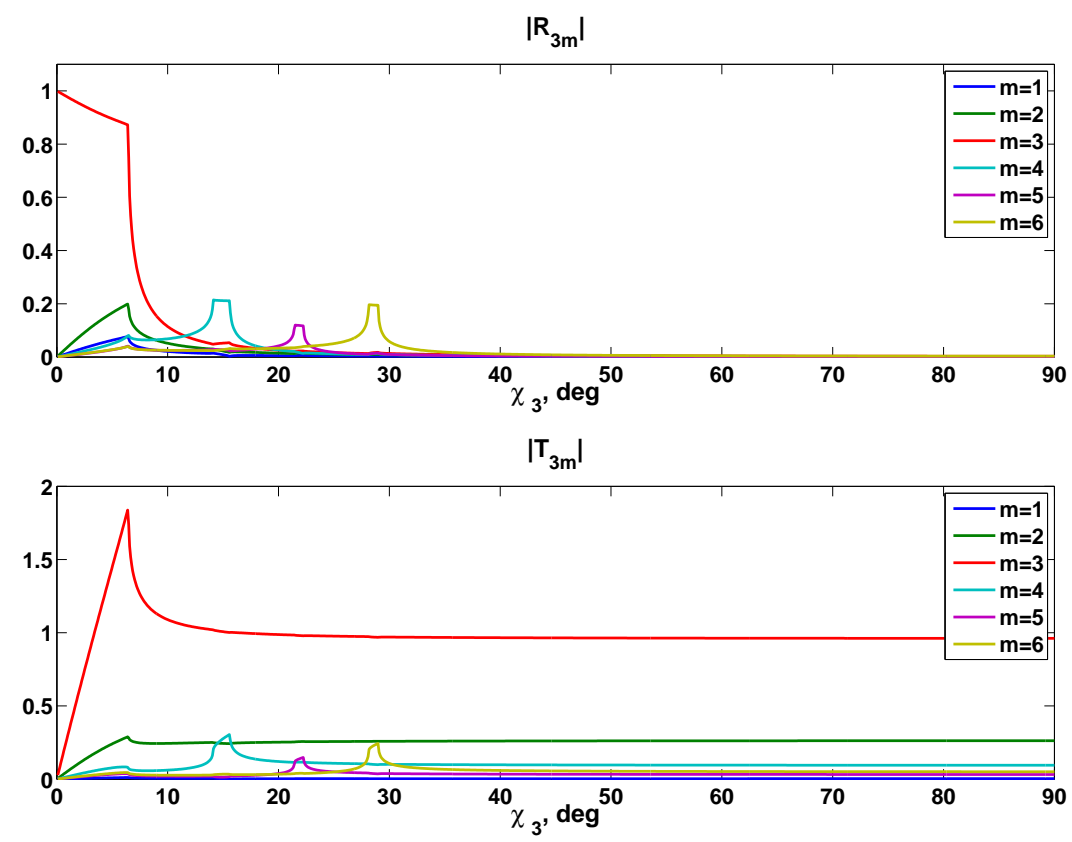

Figure 5-7: Absolute values of the plane wave coupled reflection coefficient $R_{n m}$ (top panel) and transmission coefficient $T_{n m}$ (bottom panel) for incident mode three $(n=3)$ and frequency $100 \mathrm{~Hz}$ for a sharp interface between background waveguide $\operatorname{model}\left(c_{0}=1500 \mathrm{~m} / \mathrm{s}, c_{m l}=1530 \mathrm{~m} / \mathrm{s}, c_{\text {bot }}=1800 \mathrm{~m} / \mathrm{s}, \rho_{0}=1000 \mathrm{~kg} / \mathrm{m}^{3}, \rho_{\text {bot }}=2000\right.$ $\mathrm{kg} / \mathrm{m}^{3}, H_{\text {bot }}=80 \mathrm{~m}$ and $D=15 \mathrm{~m}$ ) and a perturbation due to a twenty five meter amplitude internal wave.
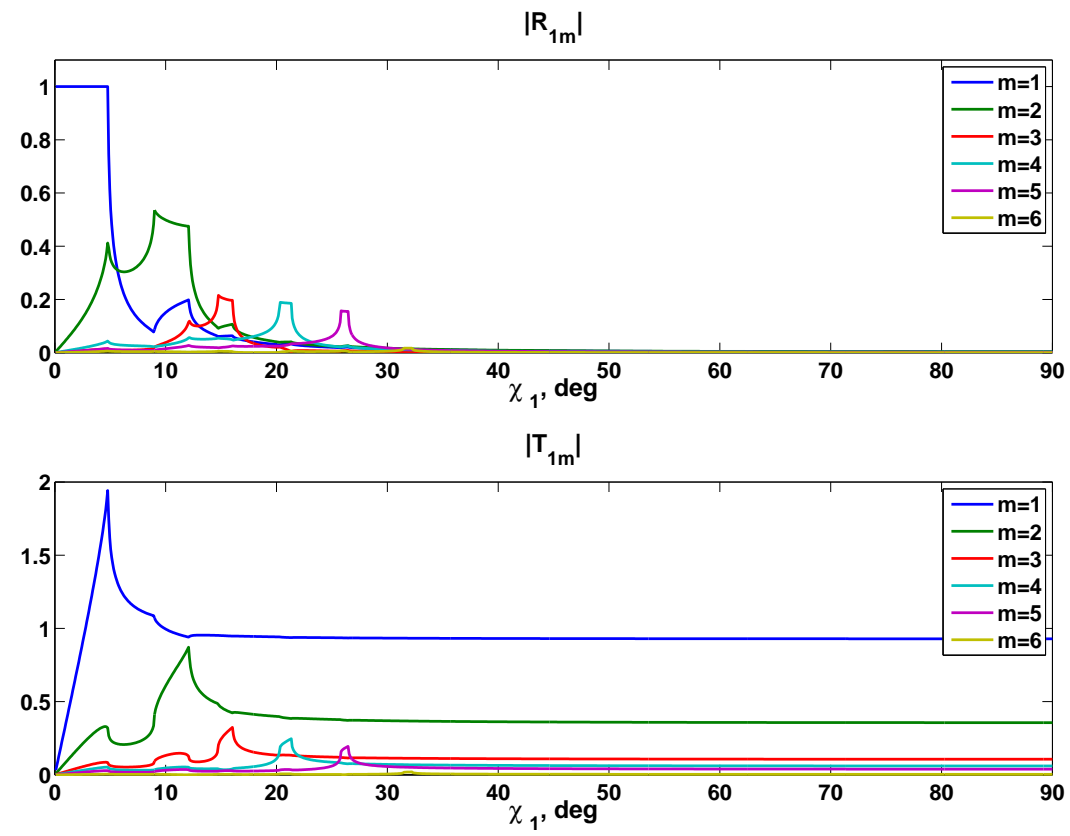

Figure 5-8: Same as in Fig. 5-7, but for mode one incident. 
Figure 5-8 illustrates the same scenario, but for mode one incident. Unlike previous example, incident mode one in this case is perfectly reflected at grazing angles below its critical value of 5.9 degrees, since all other modes at these grazing angles have imaginary $y$-components of horizontal wavenumber in both half spaces $\mathrm{A}$ and $\mathrm{B}$ and are therefore evanescent. Both examples show relatively constant mode coupling transmission coefficients for the wide range of steep grazing angles. Reflection coefficients are negligibly small in this grazing angle range.

Similar analyses for internal waves with amplitudes of up to forty meters and bottom waves and surfaces waves with amplitudes of up to four meters for the frequency range $100-500 \mathrm{~Hz}$ showed that magnitude of reflection coefficient for any incident mode $n$ as well as for any coupled mode pair $(n, m)$ is less than 0.01 for the wide range of steep grazing angles (typically 35 - 90 degrees). Therefore, backscattering of the normal modes from the types of waves we consider is believed to be small in our applications.

\subsubsection{Point Source Solution}

Having solved the mode coupling problem at a single sharp interface for a single incident modal plane wave, it is possible to derive the acoustic pressure field on both sides of the sharp interface due to a point source located in region $\mathrm{A}$ at $\left(0, y_{s}, z_{s}\right)$. By applying the one-dimensional inverse Fourier transform (I.F.T.) operator,

$$
\text { I.F.T. }\{\cdot\}=\frac{1}{2 \pi} \int_{-\infty}^{\infty}\{\cdot\} e^{-i k_{x} x} d x
$$

to both sides of Eq. (3.33), we get a two-dimensional separable Helmholtz equation:

$$
\rho \frac{\partial}{\partial y}\left[\frac{1}{\rho} \frac{\partial p}{\partial y}\right]+\rho \frac{\partial}{\partial z}\left[\frac{1}{\rho} \frac{\partial p}{\partial z}\right]+\left[\frac{\omega^{2}}{c^{2}}-k_{x}^{2}\right] p\left(k_{x}, y, z\right)=-\frac{\delta\left(y-y_{s}\right) \delta\left(z-z_{s}\right)}{2 \pi}
$$

As before, we start with considering a portion of acoustic energy due to the excitation of a single mode. The solution of Eq. (5.13) for an excited mode $n$ can be formally 
written as

$$
\tilde{p}_{n}\left(k_{x}, y, z\right)=\left\{\begin{array}{l}
\sum_{m=1}^{M}\left[\Phi_{n m}^{0+} e^{i k_{y m}^{0}\left(k_{x}\right) y}+\Phi_{n m}^{0-} e^{-i k_{y m}^{0}\left(k_{x}\right) y}\right] \Psi_{m}^{0}(z)+\tilde{p}_{n}^{s}\left(k_{x}, y, z\right), y \geq 0, \\
\sum_{m=1}^{M}\left[\Phi_{n m}^{1+} e^{i k_{y m}^{1}\left(k_{x}\right) y}+\Phi_{n m}^{1-} e^{-i k_{y m}^{1}\left(k_{x}\right) y}\right] \Psi_{m}^{1}(z), y<0,
\end{array}\right.
$$

where the single mode free-field source term $\tilde{p}_{n}^{s}$ is obtained by applying the I.F.T. operator to both sides of Eq. (3.14) and leaving the mode $n$ term only,

$$
\tilde{p}_{n}^{s}\left(k_{x}, y, z\right)=\frac{i}{4 \pi \rho\left(z_{s}\right)} \Psi_{n}^{0}\left(z_{s}\right) \Psi_{n}^{0}(z) \frac{e^{i k_{y n}^{0}\left(k_{x}\right)\left|y-y_{s}\right|}}{k_{y n}^{0}\left(k_{x}\right)}
$$

$\Phi_{n m}^{0(1)+}$ and $\Phi_{n m}^{0(1)-}$ are the amplitudes of mode $m$ coupled from the incident mode $n$ and traveling in the positive and negative directions of $y$-axis respectively in region A (B). The Sommerfeld radiation condition requires that

$$
\begin{aligned}
& \Phi_{n m}^{0-}=0, m=1 . . M \\
& \Phi_{n m}^{1+}=0, m=1 . . M .
\end{aligned}
$$

It follows from the boundary conditions and Eqs. (5.10) that

$$
\begin{aligned}
\Phi_{n m}^{0+}\left(k_{x}\right) & =\frac{i}{4 \pi \rho\left(z_{s}\right)} \frac{\Psi_{n}^{0}\left(z_{s}\right) e^{i k_{y n}^{0} y_{s}}}{k_{y n}^{0}} R_{n m}\left(\chi_{n}\right), \\
\Phi_{n m}^{1-}\left(k_{x}\right) & =\frac{i}{4 \pi \rho\left(z_{s}\right)} \frac{\Psi_{n}^{0}\left(z_{s}\right) e^{i k_{y n}^{0} y_{s}}}{k_{y n}^{0}} T_{n m}\left(\chi_{n}\right) .
\end{aligned}
$$

Acoustic pressure as a function of $x$ is obtained by applying the one-dimensional Fourier Transform (F.T.) operator,

$$
F . T .\{\cdot\}=\int_{-\infty}^{\infty}\{\cdot\} e^{i k_{x} x} d x
$$

to both sides of Eq. (5.14). Using Eqs. (5.17) and (5.16), the Fourier integral is evaluated for each coupled mode $m$ at its stationary points $k_{x}^{s}(n, m)$. As a result, the acoustic pressure due mode $n$ excited at the source location approximates to 


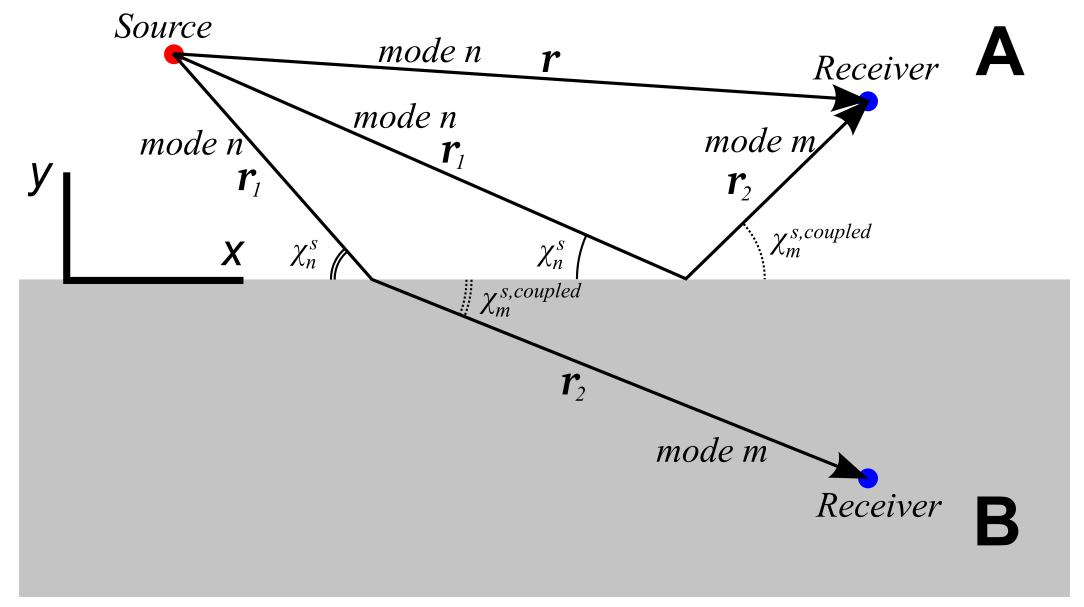

Figure 5-9: Geometrical approximation of the coupled mode horizontal reflection from and transmission through the sharp interface.

$$
p_{n}(x, y, z) \approx \begin{cases}\frac{i}{4 \rho\left(z_{s}\right)} \Psi_{n}^{0}\left(z_{s}\right) \Psi_{n}^{0}(z) H_{0}^{(1)}\left(k_{r n}^{0} r\right)+ & \\ +\frac{\Psi_{n}^{0}\left(z_{s}\right) e^{i \pi / 4}}{\rho\left(z_{s}\right) \sqrt{8 \pi}} \sum_{m=1}^{M} R_{n m}\left(\chi_{n}^{s}\right) \Psi_{m}^{0}(z) \frac{\exp \left\{i\left(k_{r n}^{0} r_{1}+k_{r m}^{0} r_{2}\right)\right\}}{\left(k_{r n}^{0} r_{1}+\left(\frac{k_{y n}^{0}}{k_{y m}^{0}}\right)^{2} k_{r m}^{0} r_{2}\right)^{1 / 2}}, \quad y \geq 0, \\ \frac{\Psi_{n}^{0}\left(z_{s}\right) e^{i \pi / 4}}{\rho\left(z_{s}\right) \sqrt{8 \pi}} \sum_{m=1}^{M} T_{n m}\left(\chi_{n}^{s}\right) \Psi_{m}^{1}(z) \frac{\exp \left\{i\left(k_{r n}^{0} r_{1}+k_{r m}^{1} r_{2}\right)\right\}}{\left(k_{r n}^{0} r_{1}+\left(\frac{k_{n}^{0}}{k_{y m}}\right)^{2} k_{r m}^{1} r_{2}\right)^{1 / 2}}, & y<0,\end{cases}
$$

where

$$
\chi_{n}^{s}(m)=\cos ^{-1} \frac{k_{x}^{s}(n, m)}{k_{r n}^{0}}
$$

is the grazing angle of mode $n$ coupled into mode $m$ for the stationary value $k_{x}^{s}(n, m)$, $r$ is the distance between the source and the receiver, $r_{1}(n, m)$ and $r_{2}(n, m)$ are the lengths of the two-leg refracted eigenray connecting the source and the receiver such that

$$
r_{1}=\frac{y_{s}}{\sin \chi_{n}^{s}(m)}, \quad r_{2}=\frac{|y|}{\sin \chi_{m}^{s, \text { coupled }}(n)},
$$


and

$$
\chi_{m}^{s, \text { coupled }}(n)=\left\{\begin{array}{l}
\cos ^{-1} \frac{k_{x}^{s}(n, m)}{k_{r m}^{0}}, y \geq 0, \\
\cos ^{-1} \frac{k_{x}^{s}(n, m)}{k_{r m}^{1}}, y<0
\end{array}\right.
$$

is the grazing angle of the reflected (or transmitted) mode $m$ coupled from the incident mode $n$ for the stationary point $k_{x}^{s}(n, m)$. Due to linearity of the governing Helmholtz equation (3.33), total acoustic pressure field excited by a point source is equal to a sum of its components due to each excited mode:

$$
p(x, y, z)=\sum_{n=1}^{M} p_{n}(x, y, z) .
$$

Equations (5.19) and (5.23) represent a geometrical acoustic approximation to coupled mode propagation in the horizontal plane. Once the eigenray for the mode pair $(n, m)$ is found from Eqs. (5.20) - (5.22), the acoustic pressure is easily computed. Figure 5-9 schematically illustrates the direct and reflected eigenrays for the source and receiver located on one side of the sharp interface (the first and second terms in Eq. (5.19) respectively for $y \geq 0$ ), and the transmitted eigenray for the source and the receiver located on the opposite sides of the sharp interface for the mode pair $(n, m)$.

Geometrical approximation of the coupled mode horizontal propagation is a useful tool that allows us to study the effects of mode coupling for more complicated environments.

\subsubsection{Horizontal Lloyd's Mirror}

In this section, we will illustrate coupled mode reflection from and transmission through the sharp interface with Eq. (5.19). As before, we use the background water column model from previous examples and its perturbation due to a twenty-five meter internal wave. Figure 5-10 shows normal mode amplitudes for the incident field consisting entirely of mode three due to a point source with coordinates $\left(x_{s}, y_{s}\right)=(0,500)$ 
m and frequency of $100 \mathrm{~Hz}$. Mode three has a unit amplitude at one meter distance from the source $(0 \mathrm{~dB}$ re $1 \mathrm{~m})$. The panel with mode three receptions shows an interesting pattern at positive values of $y$, which is the result of the interference between direct and reflected arrivals (first and second terms of Eq. (5.19) for positive values of $y$ respectively). This pattern is known as "horizontal Lloyd's mirror" (HLM) [43]. The classical Lloyd's mirror effect $[44,45]$ is a dipole-like interference of a direct and reflected path from the sea surface path in the vertical $r-z$ plane. The HLM pattern differs from the classical Lloyd's mirror in that it happens in the horizontal plane and that reflection from the wave interface is only important at the subcritical grazing angles. In classical Lloyds mirror, reflection from the sea surface is considered perfect at all vertical grazing angles. Another difference is the cylindrical spreading in the HLM as opposed to the spherical spreading in the classical Lloyd's mirror. Recent work by McMahon [41] provides theoretical studies of the HLM for straight and curved nonlinear internal wave fronts under the adiabatic approximation. Badiey et. al. recently demonstrated experimental evidence of the HLM effect after careful processing of the SW06 data $[46,15]$. Our goal in this section is to study the effects of mode coupling accompanying the HLM.

By looking at the amplitudes of the other modes, we first notice that the most of the coupled energy is concentrated in neighboring modes two and four. This is consistent with the theoretical results by Tindle $[42,37]$ showing that the coupling strength between mode pair $(m, n)$ for small waveguide perturbations is proportional to $\left[k_{r m}^{2}-k_{r n}^{2}\right]^{-1}$. The strongest mode coupling between neighbor modes was also ordinarily observed for all three types of waves within wide range of amplitudes, via our numerical modeling in Section 5.6. Coupled mode energy transmitted through the interface has an interesting angular distribution. Maximum amplitude of all coupled modes is in the direction parallel to the $y$-axis (perpendicular to the sharp interface). Coupled modes with numbers higher than the incident mode number show a gradual decrease of the transmitted field amplitude with decreasing grazing angle (mode four in our example). Amplitudes of the transmitted lower order coupled modes initially decrease with grazing angle, followed by a narrow angular range of slightly increased 
amplitude (note the uneven decrease of the amplitude of the transmitted mode two as its grazing angle decreases in Fig. 5-10). This beam with increased modal amplitude corresponds to the subcritical grazing angles of incident mode three, and illustrates the non-perfect reflection of the initial mode three at its subcritical grazing angles.

Another interesting feature to notice from Fig. 5-10 is the coupled reflection from the sharp interface. Amplitudes of both modes two and four show distinct reflected beams. The angular spectrum of these beams is narrow, which is also shown by the relatively narrow spikes of the coupled reflection coefficients in Fig. 5-7. The maximum amplitude for the reflected coupled modes in this example is approximately $12 \mathrm{~dB}$ weaker than the amplitude level of the HLM pattern. As was noticed in the previous section and illustrated above, reflection coefficient $R_{n n}$ has less than unity magnitude for $n>1$, even at subcritical grazing angles, caused by the energy leakage into both transmitted and reflected coupled modes. It was concluded during our numerical simulations that this leakage is small compared to the mean amplitude in the HLM pattern of the incident mode. For comparison, Fig. 5-11 shows plots similar to Fig. 5-10, but for mode one incident. Mode one is the lowest order mode and is perfectly reflected below its critical angle as shown in Fig. 5-8. The locations of the maxima and minima of the modal amplitudes in the HLM patterns are different for these cases, but the values of the maximum amplitudes are nearly equal.

We again note that the SIA gives overestimated values of the mode coupling strength, and that the examples in Figs. 5-7 - 5-11 are shown for qualitative illustration of the mode coupling physics. The actual importance of the mode coupling effects will be evaluated numerically in Section 5.6 for more realistic smooth wave shapes. 

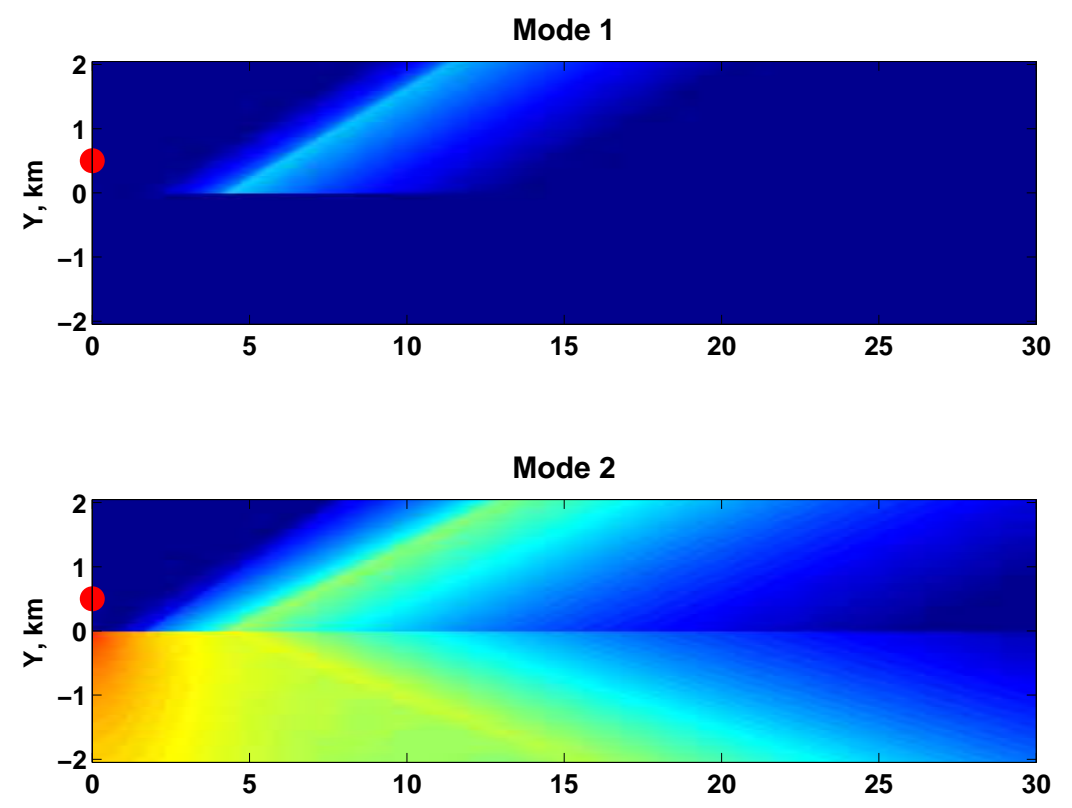

$\mathrm{dB}$
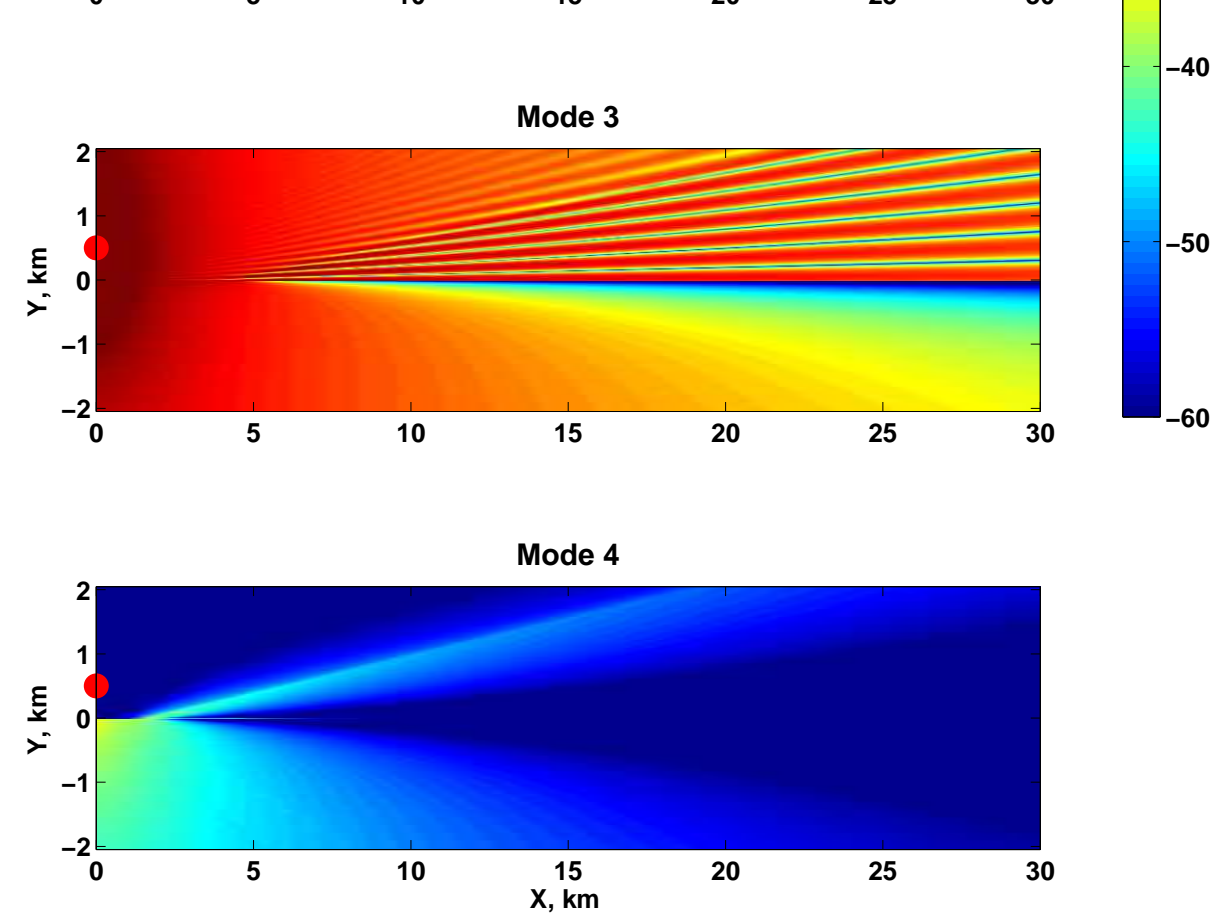

Figure 5-10: Modal amplitudes for the case of mode three incident from a point source located at $\left(x_{s}, y_{s}\right)=(0,500) \mathrm{m}$. There is a twenty five meter amplitude infinitely wide internal wave with its sharp interface along $y=0$. 
Mode 1
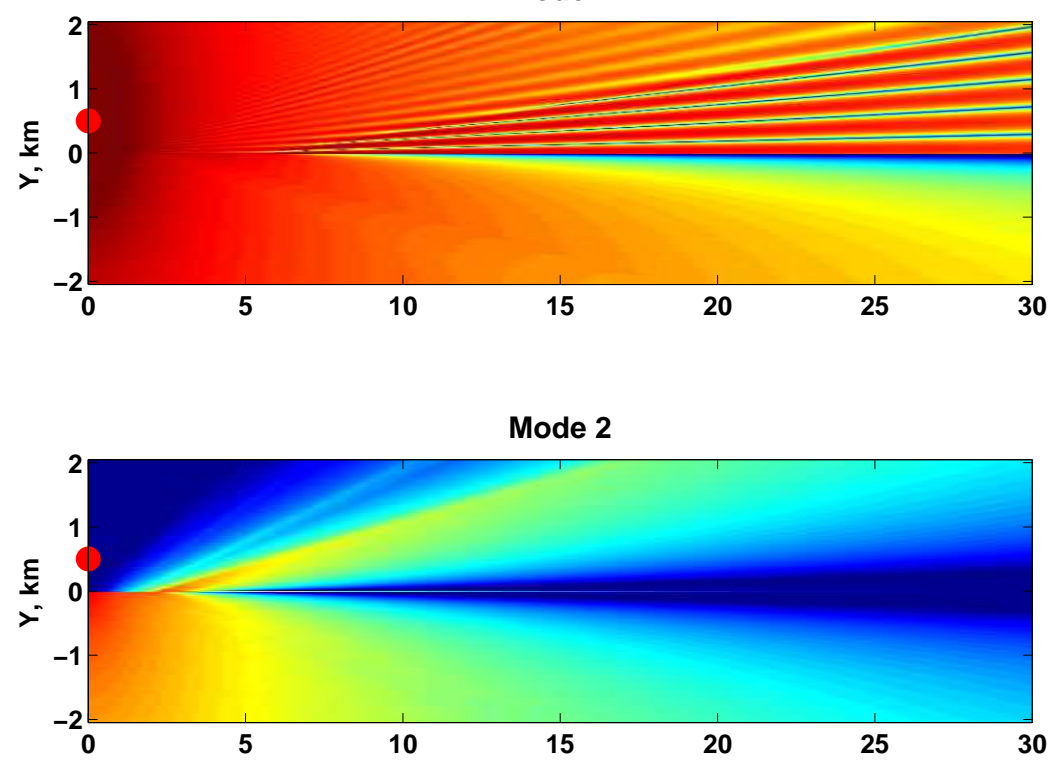

Mode 3

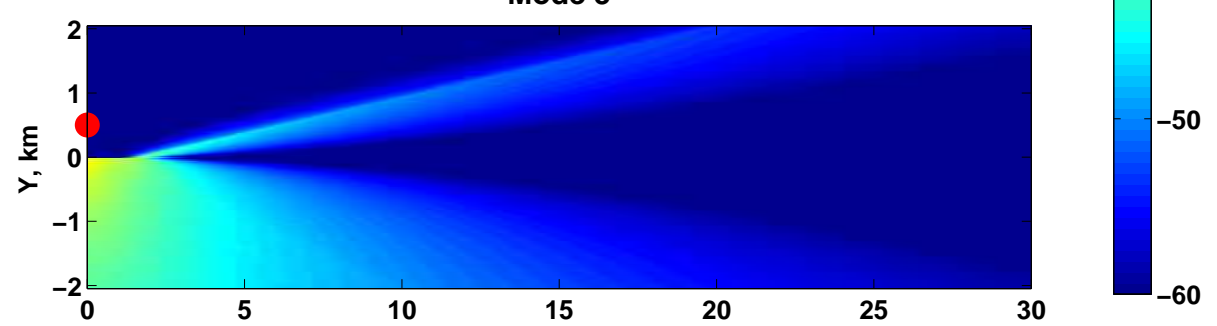

Mode 4

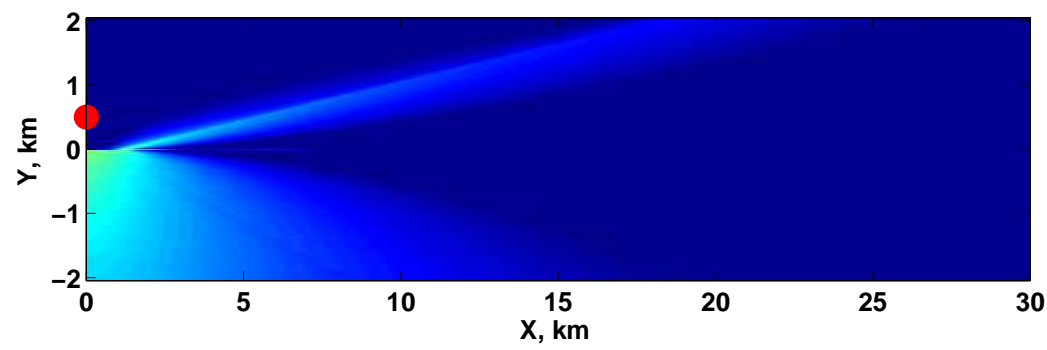

Figure 5-11: Same as in Fig. 5-10, but for the mode one incident. 


\subsection{Mode Coupled Reflection and Transmission Through SIA Wave}

Let us return to SIA waves with finite width $W$ (Fig. 5-4). Addition of the second sharp interface provides another vertical discontinuity, and mode coupling becomes a more complicated problem. We first wish to understand how the width of the SIA wave alters the HLM pattern and the amplitudes of the transmitted modes as compared to the single sharp interface studied above. As before, a single mode incident from a point source is considered. We noticed in the previous section that mode coupling is strong at steep grazing angles and weak at shallow grazing angles. It was also concluded from the reflection coefficient analysis that at steep grazing angles, backscattering of incident and coupled modes is negligibly small for all three types of waves. We will follow the logic of dividing the propagation regimes into angular sectors [39] as shown in Fig. 5-3, but will use three sectors instead: steep, shallow and intermediate grazing angles. In the first regime, mode coupling is dominant and backscattering is neglected; in the second regime, adiabatic propagation with horizontal refraction is assumed dominant and mode coupling is neglected; in the third (transition) regime, intermediate grazing angles suggest that both horizontal refraction and mode coupling should be considered.

\section{A. Steep horizontal grazing angles}

Coupled mode propagation perpendicular to the SIA internal wave crest directions was studied in detail by Duda and Preisig [4] among others. We will discuss two major effects of the coupling physics from their study: coupled cancellation and transparent resonance. To begin, consider incident mode $n$ partially coupled into mode $m$ at the front interface of the SIA wave (Fig. 5-12). In the cancellation regime, the wave width $W$ is short enough so that the relative phase difference between modes $n$ and $m$ does not change significantly as they reach back interface of the wave, and "uncoupling" occurs. In the transparent resonance regime, wave width is such that the phase difference between modes $n$ and $m$ changes by an integer multiple of $2 \pi$ as 


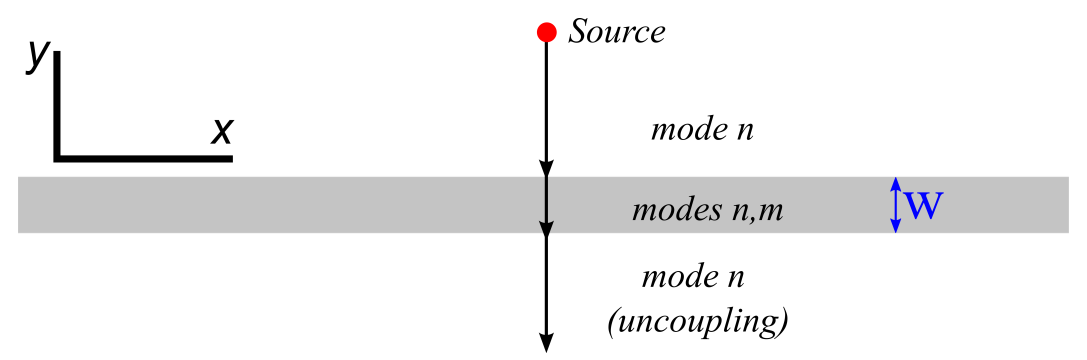

Figure 5-12: Mode coupling diagram for normal incidence of mode $n$ upon a SIA wave. Mode $n$ partially couples into mode $m$ at the front interface. At a certain resonant width of the wave, mode $m$ uncouples back into mode $n$ at the back interface.

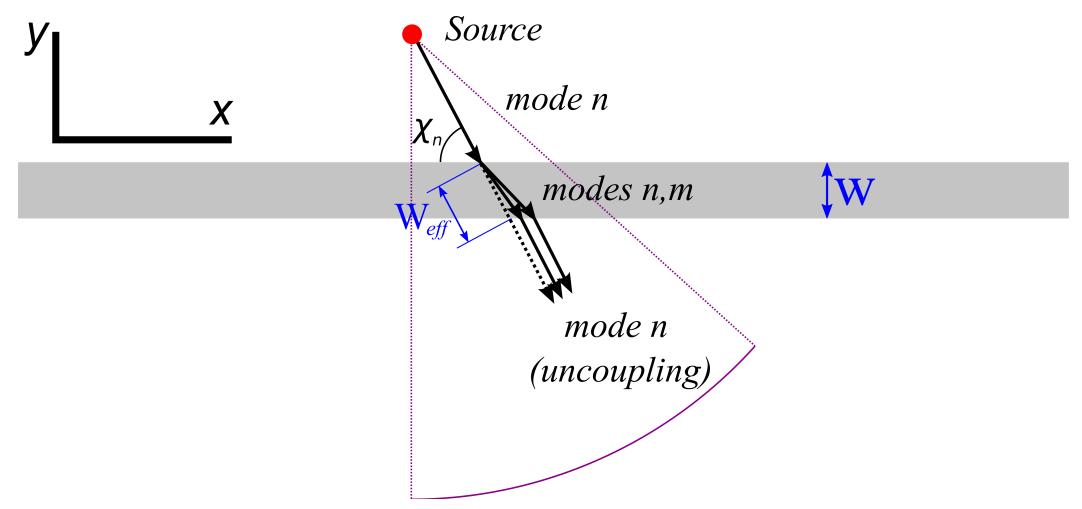

Figure 5-13: Mode coupling diagram for mode $n$ incident upon a SIA wave a with steep (other than normal) grazing angle. Weak refraction of the coupled modes at these angles makes both broken-line rays coincide with each other and with a straight (dashed) line. If one measures the modal intensities behind the wave along the arc of steep grazing angles (magenta line), significant fluctuations will be noticed as the effective width of the wave passes through its resonant values.

they travel across the wave:

$$
W^{r e s} \approx \frac{\pi l}{\left|k_{r n}^{1}-k_{r m}^{1}\right|}, \quad l=1,2, . .
$$

As a result, energy of the mode $n$ coupled into mode $m$ at the front sharp interface uncouples back into mode $n$ at the back interface, similar to the cancellation regime. Since only few significant (sometimes also called dominant) modes [4, 38] carry most of the coupled energy within the SIA and smooth-shaped waves, uncoupling of the mode $m$ back into mode $n$ will result in almost total uncoupling of mode $m$ if either of modes $n$ or $m$ is dominant.

For steep grazing angles, other than normal, it was shown for our three wave 
types (with the amplitudes and acoustic frequencies considered in the present work), that horizontal refraction of coupled modal rays as governed by Eq. (5.22) is not significant for grazing angles greater than 35-40 degrees. Interpreted another way, the ray of mode $n$ coupled into mode $m$ inside the wave coincide with the ray of mode $n$ coupled to itself inside the wave (Fig. 5-13) and both of them lay on almost a straight line. Therefore, the cancellation regime between initial mode $n$ with grazing angle $\chi_{n}$ and mode $m$ happens when the projection of the wave width onto the ray direction (effective wave width $W^{e f f}$ ) satisfies the resonance condition:

$$
W^{r e s, e f f} \equiv \frac{W}{\sin \chi_{n}} \approx \frac{\pi l}{\left|k_{r m}^{1}-k_{r n}^{1}\right|}, \quad l=1,2, . .
$$

Both the cancellation regime and the transparent resonance regimes play important roles in the intensity fluctuations behind a wave or a train of waves. If one measures the modal amplitudes behind a wave along a circle arc centered at the source position (solid magenta line in the figure), significant fluctuations are expected as the effective wave width passes through its resonant values.

\section{B. Shallow horizontal grazing angles}

At shallow grazing angles (typically below 10 degrees in our examples, and it could be even less for smooth wave shapes), horizontal refraction is important, and mode coupling is weak. For understanding the physics of the most noticeable effects, we thus neglect mode coupling and assume an adiabatic single mode refraction at the SIA wave interfaces for these angles. As we have seen, below critical angle $\chi_{c r i t, n}$, most of the energy is reflected by the infinitely wide SIA wave (and under the adiabatic approximation, total reflection occurs). Let us now return to the horizontal refraction equation (Eq. 3.41) and apply the one-dimensional I.F.T. operator (Eq. (5.12)) to its homogeneous part:

$$
\frac{\partial^{2} \Phi_{n}\left(y, k_{x}\right)}{\partial x^{2}}+\left(k_{r n}^{2}(y)-k_{x}^{2}\right) \Phi_{n}\left(y, k_{x}\right)=0 .
$$

The horizontal wavenumber $k_{r n}(y)$ has a tophat-like shape for the SIA wave (Fig. 5-14). We consider a scenario similar to the Section 5.2 of a unit amplitude mode 


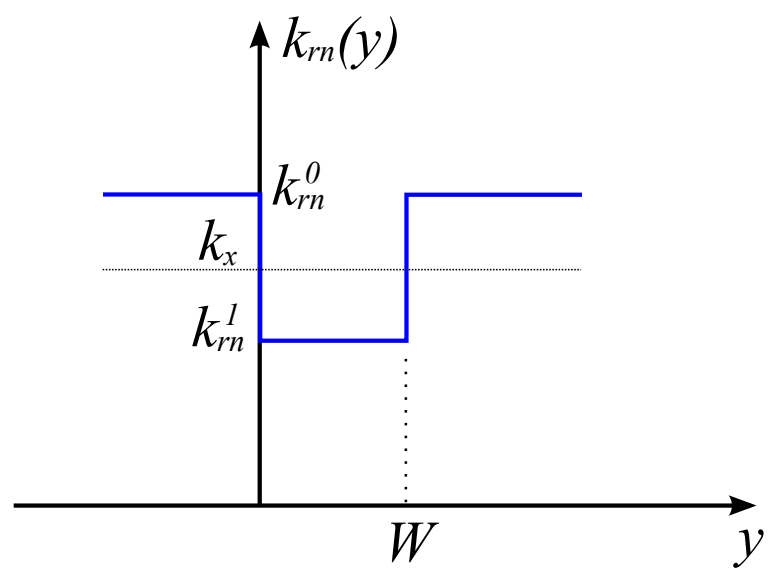

Figure 5-14: Tophat-like shape of the horizontal wavenumber in presence of the SIA wave.

$n$ with planar wave front, but incident upon the SIA wave of finite width $W$ at a subcritical grazing angle $\chi_{n}<\chi_{c r i t, n}$, and with no mode coupling. Since $k_{r n}^{0}>$ $k_{x}>k_{r n}^{1}$ at subcritical grazing angles, Eq. (5.26) represents a standard problem of particle transmission through a potential barrier in Quantum Mechanics [47, 48]. The corresponding plane wave transmission coefficient is

$$
T_{n} \approx \exp \left[-\int_{0}^{W}\left(k_{x}-k_{r n}(y)\right) d y\right]
$$

which for the SIA wave of width $W$ is

$$
T_{n} \approx e^{-W\left(k_{x}-k_{r n}^{1}\right)}
$$

One can see that the transmission coefficient above is unity at critical angle, and it rapidly decreases as the grazing angle becomes less than critical. Therefore, at the subcritical grazing angles acoustic energy propagates through the wave, and the angular spectrum of the transmitted modal amplitude is localized in a narrow angular range just below its critical value. This effect is called horizontal tunneling. In ocean acoustics, tunneling in the vertical plane was observed numerically by Jensen and Schmidt [49] when studying Gaussian beam penetration through a sediment layer at subcritical vertical grazing angles. Horizontal tunneling through internal waves has 
recently been decomposed into horizontal leaky modes of the horizontal ducts by Lin et. al. [18, 19]. Energy leakage is an inherent property of the acoustic tunneling. As a result, reflection from the wave becomes imperfect at subcritical grazing angles under the adiabatic approximation.

\section{Intermediate horizontal grazing angles}

Horizontal refraction of normal modes in our examples was shown to be considerable for grazing angles up to approximately thirty five degrees. In Section 5.2, we also noticed that at grazing angles of mode $n$ above $\chi_{c r i t, n}$, but within the range of horizontal refraction, the coefficients of the plane wave coupled reflection $R_{n m}$ and of the transmission $T_{n m}$ at the single sharp interface (Figs. 5-7 and 5-8) have their maximum values near the grazing angle

$$
\chi_{n} \approx \cos ^{-1} \frac{k_{r m}^{1}}{k_{r n}^{0}} .
$$

Above this angle, the coupled reflection coefficient $R_{n m}$ falls rapidly to zero, allowing a narrow beam of coupled mode energy to be reflected by the front wave interface. The transmitted energy of coupled mode $m$ propagates through the wave and couples again at the back interface with no significant reflection. Below this angle, coupled mode $m$ is evanescent inside the wave. Similarly to the adiabatic tunneling through the wave, below this angle the coupled energy of mode $m$ is also available for tunneling through the wave, yielding additional energy leakage through wave. With realistic smooth wave shapes, mode coupling is continuous across the wave. Therefore, we see that the tunneling of the incident mode and mode coupled tunneling are strongly interconnected effects in this range of grazing angles.

\subsection{Horizontal Acoustic Ducting}

The horizontal reflection of normal modes from wave interfaces at subcritical angles was shown to cause significant contrasts in acoustic intensity compared to the "no wave" case. Bottom sediment waves, internal waves, and ocean waves of long period 


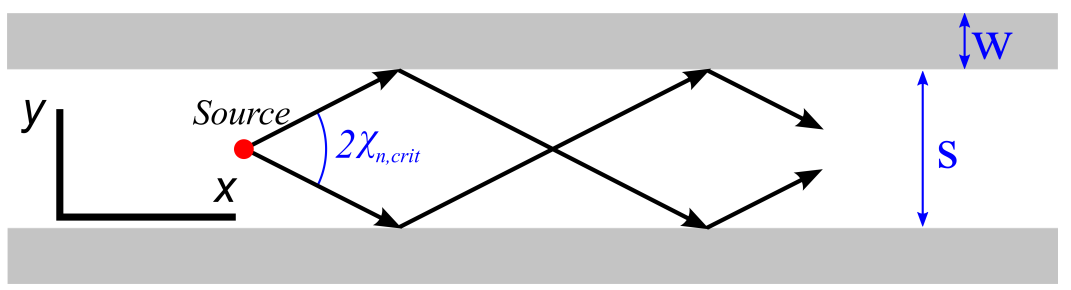

(a)

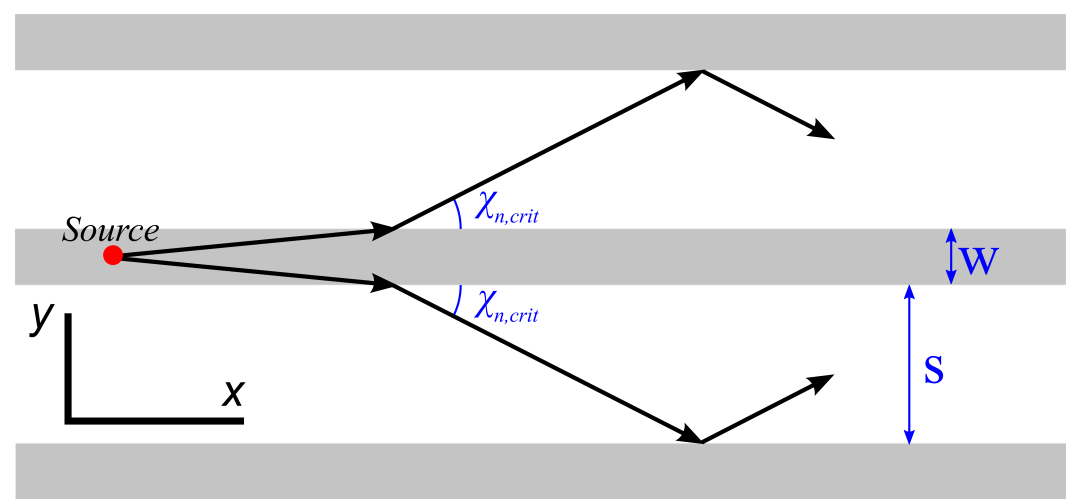

(b)

Figure 5-15: Upper panel: acoustic modal ducting for the source placed between two idealized waves. Bottom panel: acoustic modal antiducting for a source located inside an internal wave: modal rays nearly parallel to the wave crest are refracted out from the wave with the critical grazing angle.

(swell) are usually observed in groups or trains of waves. Therefore, as an internal wave or surface wave train propagates through the source location, or alternatively if a mobile acoustic source passes through a train of waves, one would expect significant intensity fluctuations in the "close-to-along-crest" directions. Figure 5-15 schematically illustrates the effects of horizontal ducting and antiducting within a train of waves. In the case of acoustic ducting, acoustic source is located between neighbor crests of depression internal waves (or surface swell troughs or bottom wave crests). This results in a part of the mode $n$ energy confined within grazing angle range $\left[-\chi_{n, c r i t}+\chi_{n, c r i t}\right]$ being trapped by these waves due to (almost) total reflection at subcritical grazing angles. Alternatively, if one puts an acoustic source inside an internal wave of depression, the horizontal ray of mode $n$ with negligibly small initial grazing angle is pushed out from the wave with grazing angle $\pm \chi_{n, c r i t}$. Also, ducting 
of the energy in between waves implies (almost) no cylindrical spreading compared to the range independent environments. Contrariwise, antiducting has stronger than cylindrical spreading.

Acoustic ducting is a major mechanism for fluctuations of the measured intensity and thus propagation to longer ranges in the along shelf direction compared to the "no waves" background scenario. Combination of ducting and antiducting events creates very significant scintillations of the acoustic intensity. These effects were first described for internal waves theoretically in [9] and numerically in [10, 8]. The first experimental observations of these strong intensity fluctuations (6-10 dB) compared to the "no waves" case, with an along wave geometry for internal waves passing over a fixed source and receiver, were made during the SWARM95 experiment [8] and described in [50]. Later, the SW06 experiment was specifically configured for measuring three-dimensional acoustic effects, and the intensity fluctuations in these directions were commonly observed in presence of internal wave trains [12].

The physics of horizontal acoustic ducting and antiducting between infinitely long parallel internal waves can be generalized to all of the three wave types. The major difference between the types in this case is in the critical angle and its dependence on the mode number, which was studied in Chapter 4. Characteristic features of the real oceanic ducts for particular wave types, such as length, directional spread, curvature, and mutual crossings will be discussed in hapters 6 and 7 .

\subsubsection{Secondary Ducting}

If internal waves were perfect reflectors at subcitical grazing angles, one would expect the intensity fluctuations in the along wave geometry to be much more than the observed 6-10 dB [8], according to Figs. 5-10 and 5-11. There exist two mechanisms allowing part of the acoustic energy to penetrate through the wave and be partially trapped in a neighbor duct: horizontal mode tunneling and coupling. We will call this type of ducting secondary ducting. Horizontal tunneling provides energy leakage through the waves at subcritical grazing angles, as was discussed in Section 5.4. When the tunneled acoustic signal encounters another wave, part of its energy is reflected, 


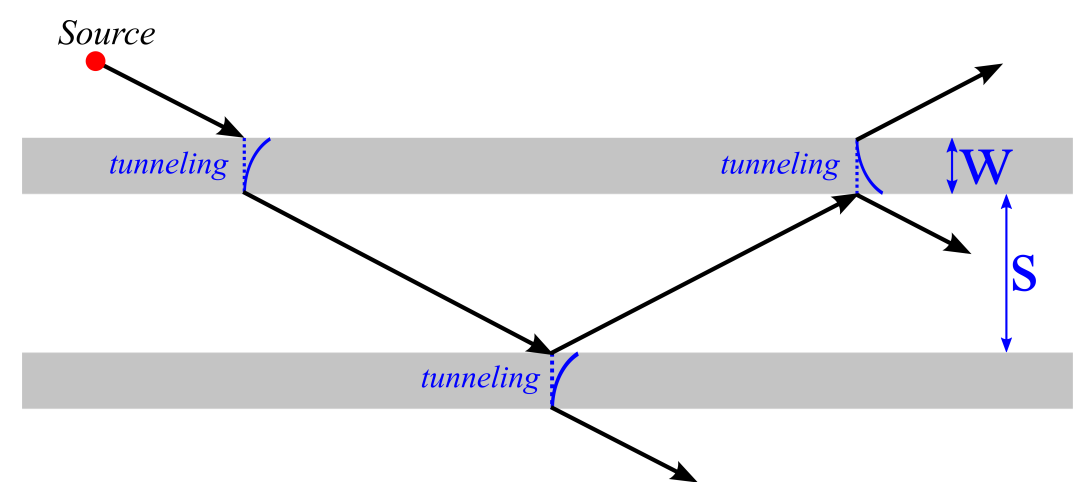

Figure 5-16: Secondary ducting due to mode tunneling. Partially ducted energy leaks out from the duct at each interaction with the waves.

and another part is tunneled further in the same manner as through the first wave. Ducted energy continues leaking at each consequent interaction with the waves (Fig. 5-16). With the adiabatic tunneled transmission coefficient given in Section 5.4, and the conservation relation between reflection and transmission coefficients,

$$
\left|R_{n}\right|^{2}+\left|T_{n}\right|^{2}=1
$$

we see that the amplitude of mode $n$ in the secondary duct after $l$ interactions with the waves is proportional to

$$
\left|\Phi_{n}\right|=T_{n} R_{n}^{l} \approx e^{-W\left(k_{x}-k_{r n}^{1}\right)}\left(1-e^{-W\left(k_{x}-k_{r n}^{1}\right)}\right)^{\frac{l}{2}}
$$

Leakage rate associated with tunneling is high at close to critical grazing angles. On the other hand, the reflection coefficient is greater further from the critical grazing angles. Therefore, angular spectrum of the acoustic field in the secondary duct narrows as $l$ increases.

Another mechanism that is responsible for secondary ducting is mode coupling. We mentioned that coupling is weak near critical grazing angles. However, if mode $n$ is incident upon a SIA wave and has higher than critical grazing angle $\chi_{n}$ such that

$$
\chi_{c r i t, n}<\chi_{n}<\cos ^{-1}\left(\frac{k_{r m}^{1}}{k_{r m}^{0}}\right)
$$




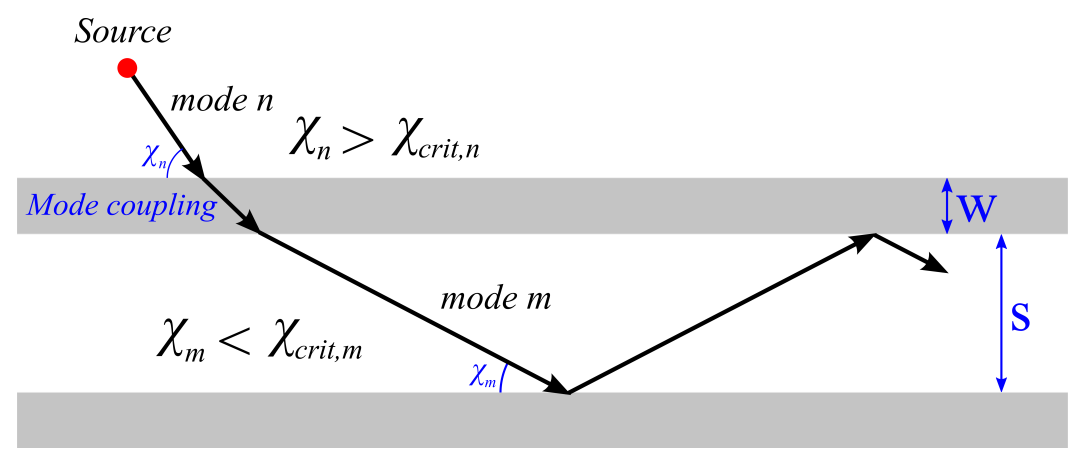

Figure 5-17: Secondary ducting due to mode coupling. Mode $n$ couples ot front or back or both interfaces of the wave into mode $m$ with higher critical angle. At certain grazing angles $\chi_{n}$, transmitted coupled energy of mode $m$ is ducted.

then weak coupling at the front or back (or both) interface of the wave into mode $m$ results in the ducting of mode $m$ (Fig. 5-17). The most favorable mode pair $(m, n)$ for this effect will satisfy the following conditions: 1$)$ wide angular range $\cos ^{-1}\left(\frac{k_{r m}^{1}}{k_{r m}^{0}}\right)-$ $\chi_{c r i t, n}$ and 2) noticeable coupling strength between modes $m$ and $n$ at these angles. We will see in the next section, however, that although tunneled secondary ducting is stronger than coupled secondary ducting, both effects are considered weak comparing to the HLM or primary horizontal ducting. On the other hand, they are the only known mechanisms that allow energy to penetrate through the wave at close to critical grazing angles and to be partially trapped in the neighbor ducts. In Chapter 6, an important application of secondary ducting will be shown for the case of crossing internal wave structures.

\subsection{Numerical Modeling}

In this section, we will evaluate the three-dimensional effects of acoustic propagation in presence of parallel internal waves, bottom sediment waves, and surface waves having smooth "canonical" shapes. Although this scenario is not fully realistic for the ocean, the following numerical examples demonstrate the quantitative importance of the fundamental mechanisms discussed above, specifically the horizontal modal refraction and mode coupling that govern acoustic propagation through real ocean waveguides. In the following two chapters, we will discuss three-dimensional acoustic 
propagation through more realistic environments that account for the geometrical and statistical properties specific to each type of wave.

Numerical methods for three-dimensional acoustics coupled mode propagation in range dependent environments are complicated and generally require a considerable amount of computing power and memory. Three-dimensional PE techniques $[51,52]$ are perhaps the most efficient ways to accomplish the task of creating a pressure field numerically. In the parallel waves application, we can eliminate the range dependence in the dimension along the wave crests. For such cases, there exists a useful Fourier transform based method $[53,54]$ that allows one to divide a three-dimensional mode coupling problem into many standard [55] two-dimensional problems (see Appendix $\mathrm{B}$ for details). This technique is favorable to us for mode calculations, since it gives the exact solution and allows all grazing angles as well as backscattering to be taken into account, unlike available PE algorithms.

The previous background waveguide model will be used for our simulations $\left(c_{0}=\right.$ $1500 \mathrm{~m} / \mathrm{s}, c_{0}=1500 \mathrm{~m} / \mathrm{s}, c_{m l}=1530 \mathrm{~m} / \mathrm{s}, c_{b}=1800 \mathrm{~m} / \mathrm{s}, \rho_{0}=1000 \mathrm{~kg} / \mathrm{m}^{3}$, $\rho_{b o t}=2000 \mathrm{~kg} / \mathrm{m}^{3}, H_{b o t}=80 \mathrm{~m}$ and $D=15 \mathrm{~m}$ in Fig. 4-1). As before, we model the waves by displacing one of the three interfaces, and then use the KRAKEN [32] normal mode code for computing modal functions and horizontal wavenumbers across the direction of the wave crests. In our analysis, the main accent will be made on the internal waves, since they were noticed to cause stronger mode coupling then other waves do. However, the main parallels and differences with the bottom sediment waves and surface waves will also be outlined. A hyperbolic secant squared wave shape is used for modeling the internal waves of depression,

$$
H_{i w}(y)=D+\Delta H_{i w}(y) \operatorname{sech}^{2}\left(\frac{y-y_{\text {wave }}}{W}\right)
$$

where $\Delta H_{i w}=25 \mathrm{~m}$ is the amplitude of the wave used in the simulations, $W=70 \mathrm{~m}$ is the width parameter, and $y_{\text {wave }}$ is the coordinate of the wave center. This wave shape is as a formal solution of the Korteweg-de-Vries equation [56] describing the propagation of nonlinear internal waves. The half amplitude width of this wave is 
130 meters. Upper panel in Fig. 5-18 shows the shape of this wave (black line). In our numerical modeling, hundred steps are used to divide the waveguide inside the wave into locally range independent sectors (blue line in the upper panel of Fig. 5-18). We limit ourselves here to a frequency of $100 \mathrm{~Hz}$, since we did not see any physical differences in the results for higher frequencies. Changes in the horizontal wavenumber across the wave with respect to background water column values, as well as the horizontal critical angles of the normal modes, are shown in the middle and bottom panels of Fig. 5-18 respectively.

\section{A. Single wave}

We start our modeling with a single internal wave centered along $y_{\text {wave }}=0 \mathrm{~m}$ and acoustic source at $x_{s}=0 \mathrm{~m}, y_{s}=500 \mathrm{~m}$. For visualizing the effects of mode coupling, we let the incident field consist of a single mode with unit amplitude at one meter distance from the source, as we did in our theoretical studies in Section 5.3.3. Figure 5-19 shows modal amplitudes for the case of incident mode one. Location of the internal wave crest is schematically shown by the white dashed line. One can clearly see the likeness of the numerical solution for the smooth wave shape to the theoretical solution for the infinitely wide SIA wave in Fig. 5-11. The HLM pattern of mode is almost identical between the two, with only a slight difference in the locations of the modal amplitude maxima and minima. We also note the similarity in the reflected beams of coupled modes two and three. However, the modal amplitudes of these beams are weaker compared to the SIA case. This internal soliton with smooth wave shape results in a $17 \mathrm{~dB}$ difference between coupled mode reflection and the reflection of the incident wave (the sharp interface of the same amplitude (Fig. 5-11) provided $5 \mathrm{~dB}$ stronger reflection of the coupled mode one). As we see from the plots, the simple sharp approximation used in previous sections is a very efficient way of studying the physics of mode coupled refraction on parallel waves. The main difference noted between the solutions is in the transmitted field, which is mainly due to the finite width of the $\operatorname{sech}^{2}$ wave compared to the infinitely wide SIA wave in Fig. 5-11. The finite width of the wave results in larger shadow zones and moves the maxima of the transmitted modal amplitudes off the $y$-axis to some grazing angle 

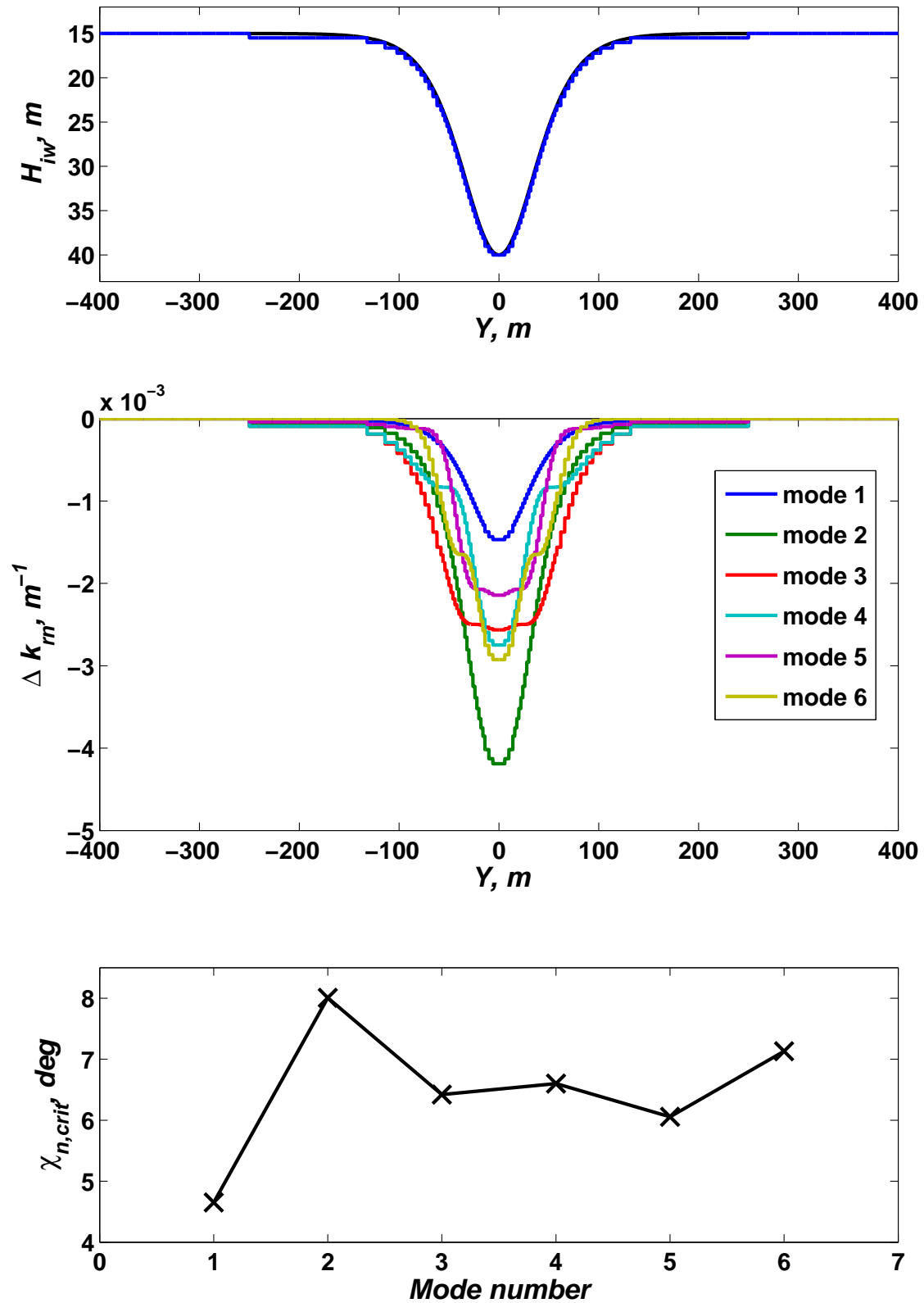

Figure 5-18: Upper panel: internal wave of depression with amplitude of $25 \mathrm{~m}$ and hyperbolic secant squared shape (black line). Half amplitude wave width is $130 \mathrm{~m}$. The blue line represents hundred steps dividing the wave into locally range independent sectors used in numerical modeling. Middle panel: changes in the horizontal modal wavenumbers across the soliton internal wave with respect to background water column values. Bottom panel: Normal mode horizontal critical angles. 
that is mode number dependent. In addition, the amplitude of mode three shows a characteristic minimum at a transmitted grazing angle of 10-12 deg. This is direct evidence of the transparent resonance studied in Section 5.4. A situation similar to the Fig. 5-19 scenario, but for incident mode three, is shown in Fig. 5-20. The panels for modes two and four illustrate distinct nulls in the coupled transmission resulting from the transparent resonance. Almost $10 \mathrm{~dB}$ of modal amplitude fluctuation due to the resonance is noticed for mode two, which is considerable. This is consistent with very strong resonance type intensity fluctuations observed in both the Yellow sea [3] and the SWARM95 [6] experiments.

Similar numerical simulations were performed for bottom sediment waves and surface waves, both with amplitude of two meters. The only major physical difference noted was in the strength of mode coupling. Internal waves often (but not always) cause a strong mode coupling between neighboring lower order modes (modes 13 in the example above), and weaker coupling of higher order neighboring modes. Contrariwise, mode coupling due to bottom or surface waves is almost not noticeable for the lower order modes and is stronger for higher order modes.

As an example, we consider a bottom sediment wave with a two meter amplitude and exactly the same wave shape as in previous example with internal wave (hyperbolic secant squared with half amplitude width of 130 meters). This particular wave shape was chosen for comparison with internal waves only (note that the shapes of the bottom sediment waves vary widely and are often not symmetric [57]). Plots in Fig. 5-21 illustrate the shape of this bottom wave (upper panel), perturbations to the modal horizontal wavenumbers (middle panel) and associated horizontal critical angles (bottom panel) respectively. The maximum critical angle for this bottom wave is eight degrees, which is nearly equal to the maximum critical angle for the internal wave example above. Figure 5-22 shows corresponding modal amplitudes for incident mode five, which is last but one propagating mode in this waveguide. Although the mode coupling due to bottom waves is stronger between higher order modes, we note the weak coupling of the incident mode with its neighbor modes four and six compared to the much stronger coupling of the lower order modes for the internal wave 

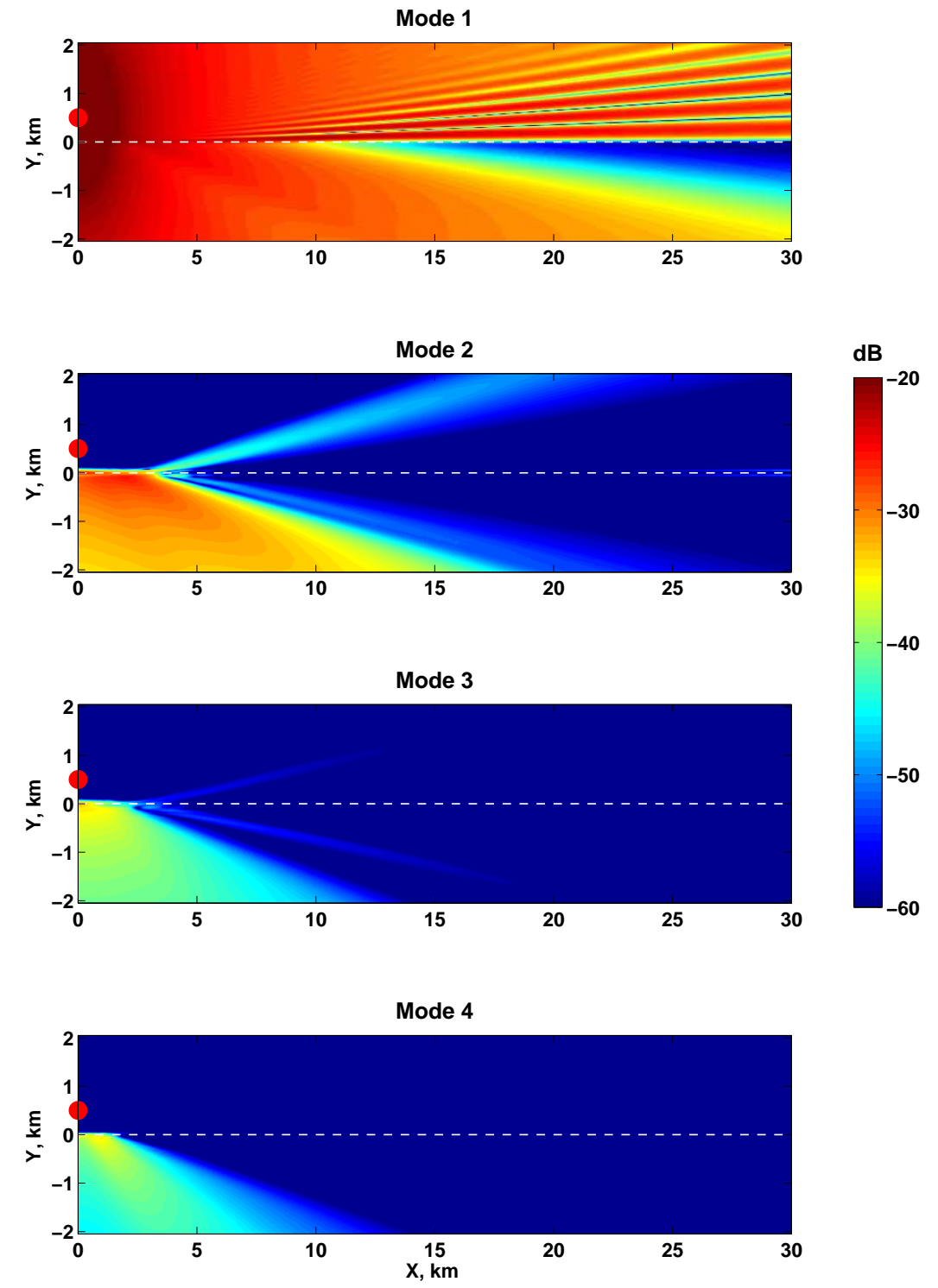

Figure 5-19: Amplitudes of modes 1-4 for the case of incident mode one with unit amplitude at one meter distance from the source and single internal wave of twenty five meter amplitude centered at $y_{\text {wave }}=0 \mathrm{~m}$. Source is located at $y_{s}=500 \mathrm{~m}$ (red circle). 

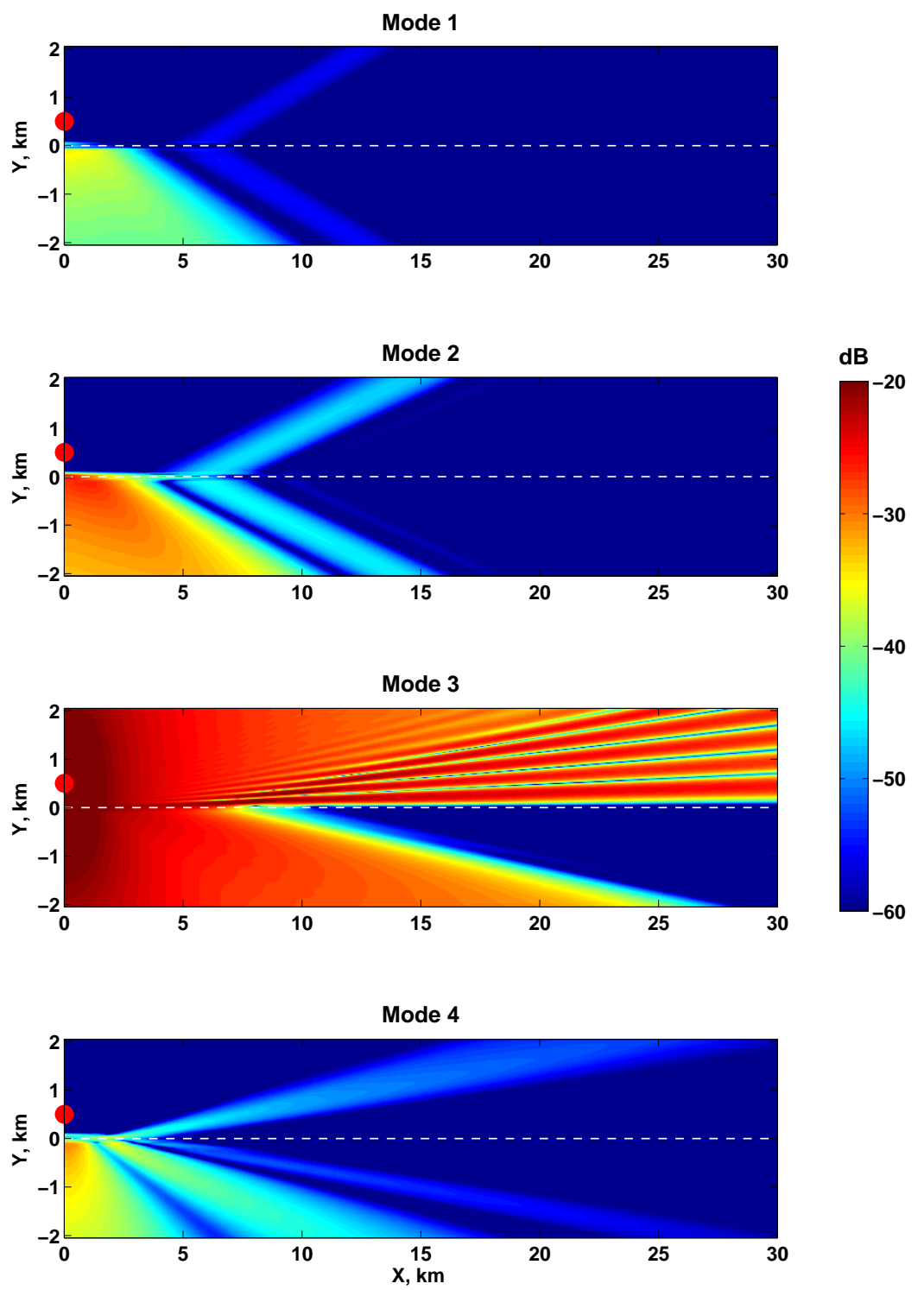

Figure 5-20: Same as in Fig. 5-19, but for incident mode three. 
case in previous example. It was concluded from our simulations that with comparable widths of the waves and associated horizontal critical angles, internal waves cause stronger mode coupling than bottom or surface waves. We note however that bottom and surface waves can have much shorter wavelength than internal waves and in such cases mode coupling may be strong. In addition to the weaker mode coupling due to bottom and surface waves, surface waves were also shown to cause modal energy leakage into the continuous spectrum rather than mode coupling. This special case will be discussed in Chapter 7 .

\section{A. Multiple waves}

In the following example we place two identical internal waves (with the same amplitude and width as in the examples above) centered at $y_{s}= \pm 300 \mathrm{~m}$. Figure 5-23 illustrates the case of horizontal ducting with an acoustic source in between the waves $\left(y_{s}=0 \mathrm{~m}\right)$ and incident mode one of unit amplitude at one meter distance from the source. The upper panel indicates a significant (12-15 dB of modal amplitude difference) amount of energy trapped between the waves. This energy travels over a long distance depending on the length $[18,19]$ and curvature [17] of the duct, since there is (almost) no cylindrical spreading associated with its propagation. Along with ducting of incident mode one, one can also note ducting of a coupled mode two, which is 15-20 dB weaker than the ducting of the initial mode one. This effect is considered weak, and likely will not be noticed or measured if all modes are excited. Of importance is the effect of tunneling seen in the upper panel. At subcritical angles, mode one energy leaks out of the duct, becoming available for the neighbor duct. As the distance from the source increases, the tunneled energy rate decreases, but we note considerable $7 \mathrm{~dB}$ difference between the tunneled and ducted modal amplitudes $15 \mathrm{~km}$ away from the source. The grazing angle of the tunneled mode is localized near its critical angle, and this is the only difference between the tunneling of the other modes.

We next relocate the source to $y_{s}=500 \mathrm{~m}$ and shift wave centers to $y_{\text {wave } 1}=0$ $\mathrm{m}$ and $y_{\text {wave } 2}=-600 \mathrm{~m}$ such that source is outside of both waves and repeat the simulation. The resultant modal amplitudes for mode one incident are shown in 

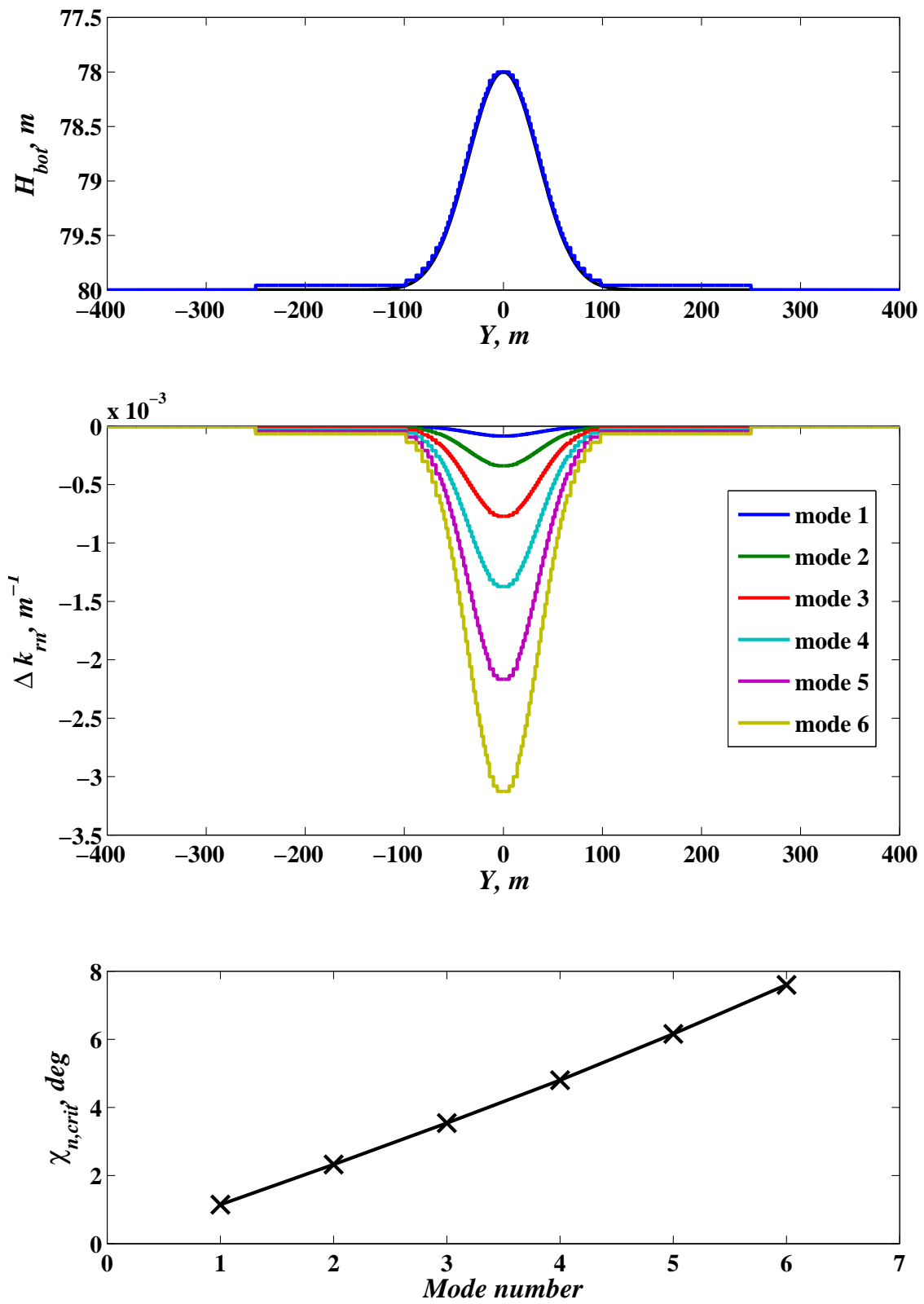

Figure 5-21: Upper panel: a bottom sediment wave of two meter amplitude with hyperbolic secant squared shape (black line). Half height width is $130 \mathrm{~m}$. The blue line represents hundred steps dividing the wave into locally range independent sectors to be used in numerical modeling. Middle panel: changes in the horizontal modal wavenumbers across the bottom sediment wave with respect to background water column values. Bottom panel: normal mode horizontal critical angles. 



Figure 5-22: Amplitudes of modes 3-6 for incident mode five with unit amplitude at one meter distance from the source and a single bottom sediment wave of two meter amplitude centered at $y_{\text {wave }}=0 \mathrm{~m}$. Source is located at $y_{s}=500 \mathrm{~m}$ (red circle). 

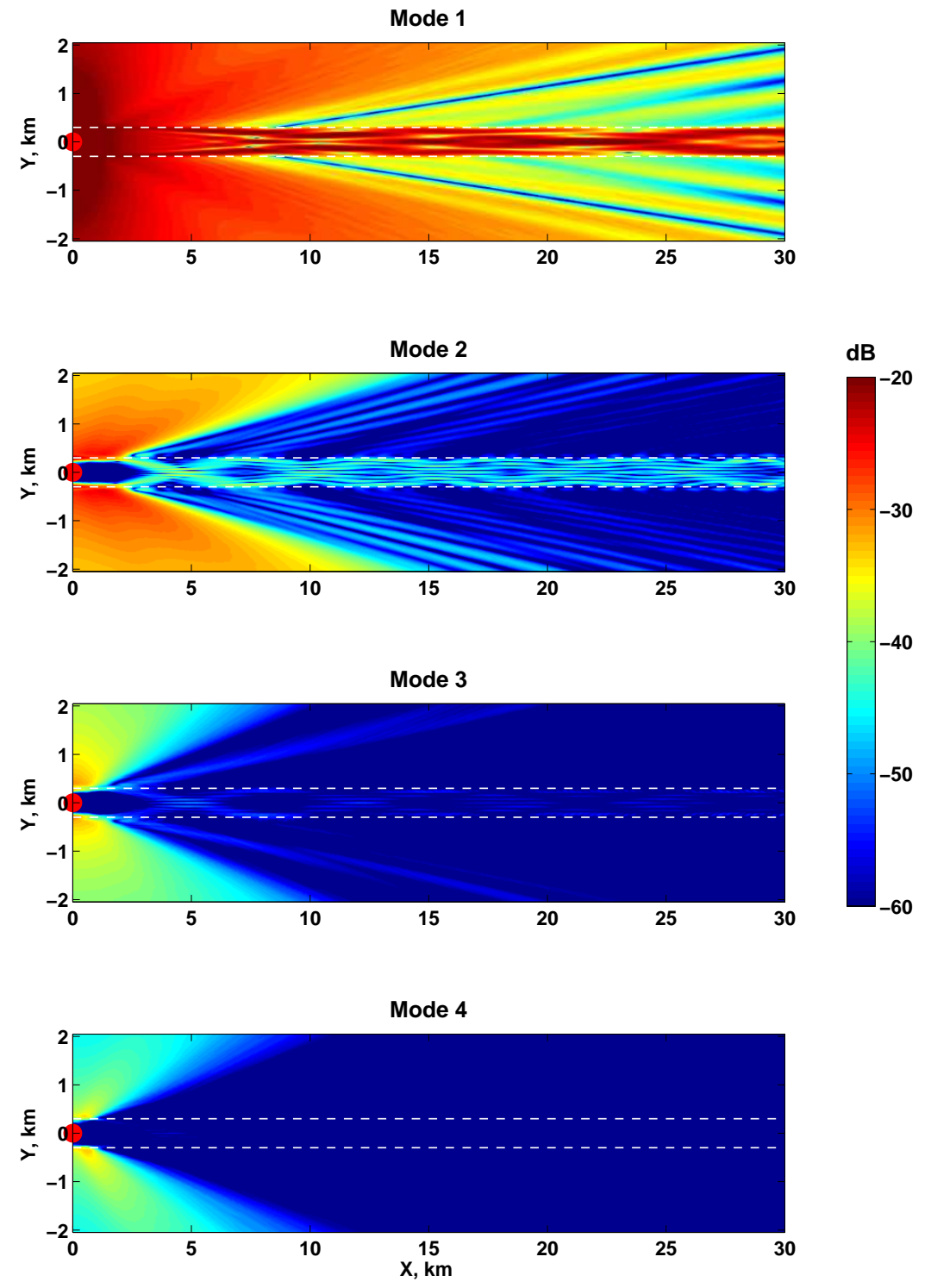

Figure 5-23: Horizontal ducting of normal mode. Acoustic source located at $y_{s}=0 \mathrm{~m}$ is in between two internal waves centered at $y_{\text {wave }}= \pm 300 \mathrm{~m}$. Incident field consists of a single mode one with unit amplitude at one meter distance from the source. 
Fig. 5-24. From the upper three panels, the effects of secondary ducting are clearly seen: tunneled ducting of incident mode one, and coupled ducting of modes two and three. Obviously, the tunneled ducting is more energetic in this case: the amplitude of tunneled mode one is nearly $10 \mathrm{~dB}$ larger than the amplitude of the coupled mode two in the secondary duct. A similar example, but for mode two incident, is plotted in Fig. 5-25. We see that in this case, the tunneled field of the incident mode two has a steeper angle and thus more reflections within the duct over the same distance (note that mode two has the greatest critical angle of 8.3 degrees). We remember that tunneled secondary ducts are associated with continuous leakage of modal energy out of the duct during each interaction with wave. Figures 5-24 and 5-25 clearly illustrate this phenomenon. Similar to the previous example, coupled secondary ducting is weak. It has been concluded from multiple numerical simulations, that mode tunneling has similar strength for all mode numbers, whereas coupled secondary ducting was noticed only for low order modes and only for internal waves. The two examples considered illustrate the strongest coupled ducting noticed. Hence, coupled ducting is not considered to be an important effect.

Amplitudes of the modes transmitted through both waves show distinct transparent resonances that are closer spaced in grazing angle and have bigger amplitude fluctuations than for the case of one wave (Figs. 5-19 and 5-20). We noticed in our simulations, that the scintillation of the modal amplitudes transmitted through the wave train initially increases with the number of waves in the train due to cumulative transparent resonances. However, after a certain number of waves in the train (five to six in our case) having slightly different amplitudes and widths, variability in the nulls and maxima in the multiple resonances results in "smearing" of the acoustic amplitude fluctuations. Fredericks et. al. [7] showed that for a fixed source and receiver in the SWARM95 experiment, situated across the mean direction of the internal wave crests, the root mean square of the log-intensity over a several hour time window was near $5.6 \mathrm{~dB}$, the statistical value expected for fully saturated ocean waveguides $[58,59]$. 

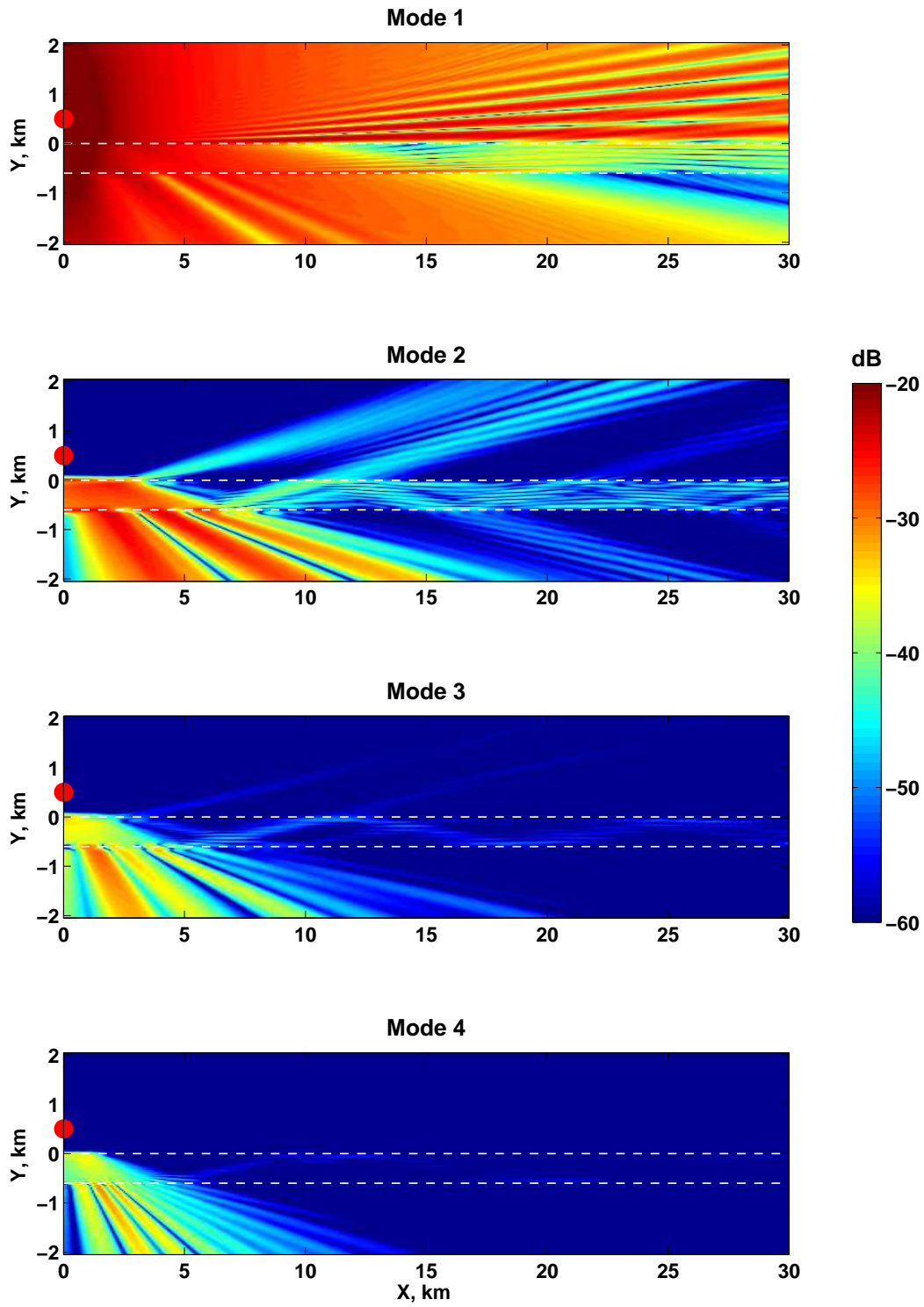

Figure 5-24: Secondary ducting. An acoustic source at $\left(x_{s}, y_{s}\right)=(0,500) \mathrm{m}$ is located just outside of the duct composed of two internal waves centered at $y_{\text {wave } 1}=0 \mathrm{~m}$ and $y_{\text {wave } 2}=-600 \mathrm{~m}$. The incident field consists of a single mode one of unit amplitude at one meter distance from the source. 

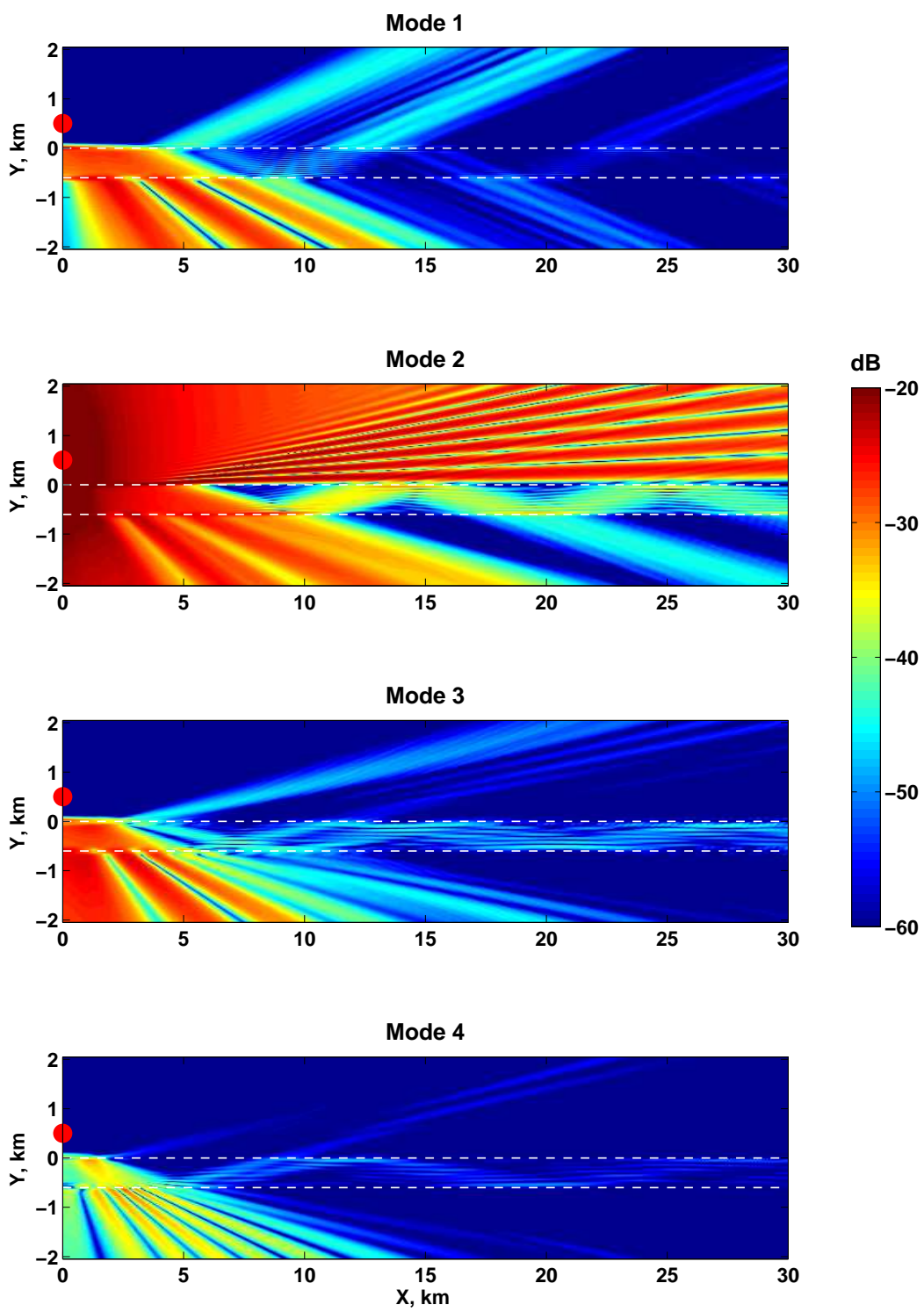

Figure 5-25: Same as in Fig. 5-24, but for incident mode two. 


\section{Chapter 6}

\section{Crossing Internal Waves}

One of the important features common for internal waves in shallow waters is the crossings of separate internal wave trains. Crossing structures are seen on almost all of the satellite synthetic aperture radar (SAR) images when internal waves are present [20]. As an example, Fig. 6-1 shows one of the SW06 site SAR images with multiple crossings of the curved internal wave trains (taken on July 23, 2006). Analysis of multiple SAR images, including this example suggests that the angle of the waves cross varies widely from 0 to 90 degrees. Although curvature of the internal wave fronts is commonly observed together with the wave crossings, in this chapter we will study the effects of three-dimensional acoustic propagation through the medium that contains crossing of straight wave trains (acoustical effects caused by the wave curvature are studied in $[41,17])$. In particular, we are interested in the interaction of the acoustic energy ducted in between waves from one train with crossing wave train. To do this, we briefly explain the governing physics of crossing ducts below and associate it with the studied effects for the parallel waves case. In Section 6.2, we will present the results of three-dimensional acoustic PE modeling for the straight internal waves crossings at various angles. 


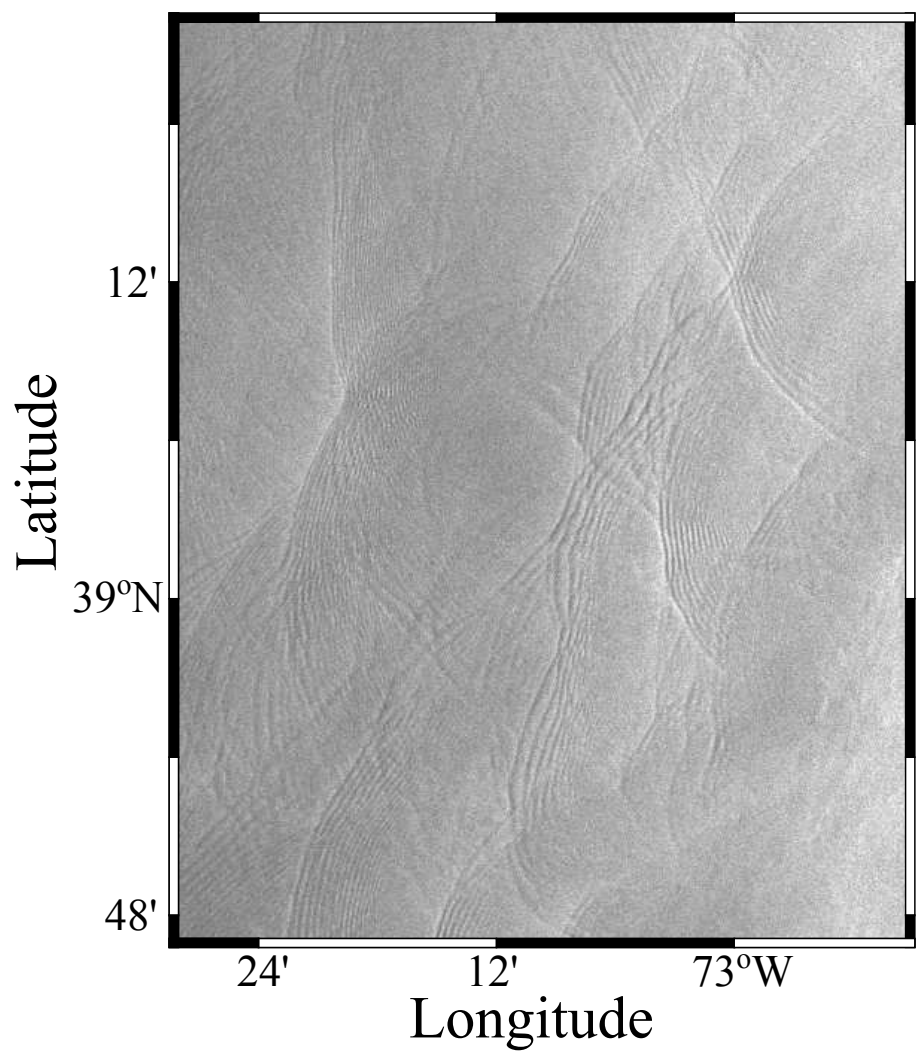

Figure 6-1: SAR image of the internal waves in the SW06 experimental area showing multiple crossings of the curved wave trains. Taken on July 23, 2006. 


\subsection{Physics of Crossing Acoustic Ducts}

The physics of crossing internal soliton waves is a complicated nonlinear oceanographic problem. When one soliton is traveling across another, strong interaction occurs, and this problem remains under active research presently. In application to low frequency acoustics, we make a rough assumption of the internal wave amplitude inside the crossings to be the maximum of two of undisturbed solitons. Taking into account a relatively small area inside interacting solitons compared to the width of acoustic ducts, we believe that for studying the first order acoustic effects in presence of such structures, our assumption is reasonable.

Consider the acoustic energy of mode $n$ horizontally trapped between two straight SIA internal waves. The grazing angles of the horizontal rays are confined within the range $\left[-\chi_{c r i t, n} . .+\chi_{c r i t, n}\right]$, where $\chi_{c r i t, n}$ is the critical horizontal angle of mode $n$ associated with these waves, as before. Now let another pair of internal SIA waves to cross the first one at angle $\gamma^{\text {cross }}$. We can divide the physics of acoustic interaction with the crossing waves into two ranges of $\gamma^{\text {cross }}$ : steep and shallow (Fig. 6-2). At steep angles of crossing (typically above $2 \chi_{c r i t, n}$ if the two pairs of waves are identical), the angle between the horizontal ray of mode $n$ and the crossing wave is steep, and mode coupling is therefore considerable. This scenario is schematically shown in the upper panel of Fig. 6-2 with red rays indicating mode coupling. There are usually more than two waves in the crossing train. Also, multiple SAR images [20] seen to indicate that crossings of the internal wave trains often happen near wave terminations. Therefore, energy of mode $n$ will be highly scattered by the crossing of these high angle waves (similar to the steep incidence upon the parallel waves in the previous chapter) and no longer ducted for steep angles of wave crossing.

At shallow crossing angles (less than $2 \chi_{c r i t, n}$ ), there exist several mechanisms that pull modal energy out of the duct by either reflection or cross-ducting. In the case of reflection, some of the modal rays hit the interface of the leading crossing wave at a subcritical angle and this part of the modal energy is (almost) totally reflected by this wave (red solid ray in the bottom panel of Fig. 6-2). After being reflected, 


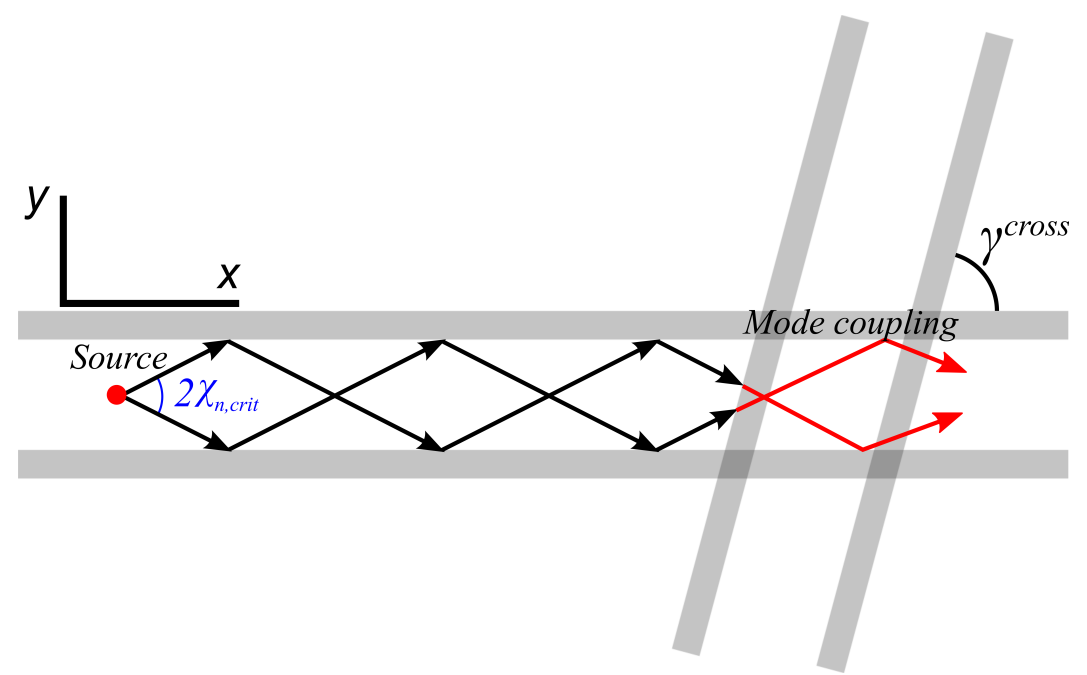

(a) Steep crossing angle

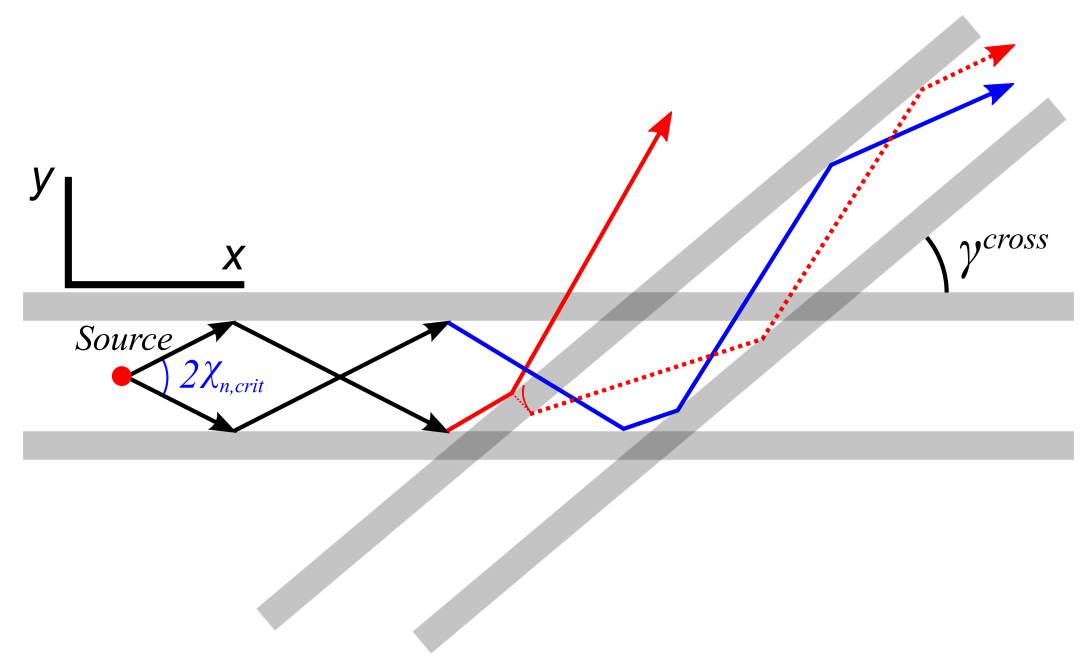

(b) Shallow crossing angle

Figure 6-2: Schematic view of ducted acoustic energy interaction with crossing waves. At steep angles of wave crossing (upper panel), mode coupling is considerable, and ducted energy is scattered by interactions with multiple waves. At shallow angles of wave crossing (bottom panel), part of the modal energy is pulled out of the duct by reflecting from the first crossing wave (red solid ray), and another part tunnels (red dashed ray) or propagates (blue ray) through the first crossing wave and becomes ducted between the first and the second crossing waves (cross-ducting). 
its grazing angle with respect to the waves of the initial duct is no longer subcritical and this part of the energy leaves the duct. As seen in the previous chapter, modal reflection at subcritical angles is not perfect: horizontal tunneling can provide the leakage through the wave. When the energy tunneled through the first crossing wave reflects from the second crossing wave, it leaves the initial duct and becomes ducted by the crossing waves, or cross-ducted (red dashed line in the same plot). Mode coupled cross-ducting is also possible (in direct analogy to the mode coupled secondary ducting), but numerical simulations below show it to be a negligible effect. Also cross-ducted are the modal rays that propagate through the first crossing wave at steeper than critical grazing angle and then are consequently reflected by the wave from the original duct and the second crossing wave (blue ray on in the plot).

Both reflection from the leading crossing wave and cross-ducting will be shown below to be important at crossing angles less than $2 \chi_{c r i t, n}$. The relative strength of these effects is shown to be dependent on the actual value of the crossing angle.

\subsection{Numerical Modeling}

In this section, we take advantage of a Cartesian three-dimensional PE acoustical propagation program [60] for handling ocean environments with crossing internal waves. This program solves one-way wave propagation PE for a point source radiating single frequency continuous wave (CW) signal and is based on a split-step Fourier technique [61]. A wide angle Thompson and Chapman propagation operator [62] is used for studying a wide range of internal wave cross angles. A very good agreement of the results obtained with this program and the exact mode coupled solution for one of the parallel waves examples considered in previous chapter is demonstrated in Appendix C.

As a background water column, we use our two-layer model with previous parameters $\left(c_{0}=1500 \mathrm{~m} / \mathrm{s}, c_{0}=1500 \mathrm{~m} / \mathrm{s}, c_{m l}=1530 \mathrm{~m} / \mathrm{s}, c_{b}=1800 \mathrm{~m} / \mathrm{s}, \rho_{0}=1000\right.$ $\mathrm{kg} / \mathrm{m}^{3}, \rho_{\text {bot }}=2000 \mathrm{~kg} / \mathrm{m}^{3}, H_{\text {bot }}=80 \mathrm{~m}$ and $D=15 \mathrm{~m}$ in Fig. 4-1). Vertical smoothing of the sound speed and density is applied as necessary for the program 
use. Internal waves from the first wave pair have an amplitude of twenty five meters and are placed parallel to the $x$-axis along $y_{\text {wave }}= \pm 300 \mathrm{~m}$, as in the previous chapter. For modeling the initially ducted modal energy, we place acoustic source in between the first pair of internal waves at $\left(x_{s}, y_{s}\right)=(0,0) \mathrm{m}$. The depth of the source is not specified here, since we use a single mode starter in our program in order to evaluate the effects of mode coupling at the wave crossings. The top panel of Fig. 6-3 shows the smoothed sound speed water column cross section along $x=0$ $\mathrm{m}$ and the smoothed density column used by the program. The second group of waves consists of a single or a pair of identical waves with the same parameters as the first pair and it crosses the first group at $x_{\text {cross }}=100 \mathrm{~km}, y_{\text {cross }}=0 \mathrm{~m}$. The large distance from the acoustic source to the wave crossing is only chosen to clearly show the effects of wave crossings on the ducted energy. There is no physical attenuation included in the model, and therefore the energy level within the duct is not affected by this distance. In our modeling, we use an acoustic frequency of $100 \mathrm{~Hz}$ and an initial field composed entirely of mode one (as before, with unit amplitude at one meter distance from the source), which showed the strongest coupling comparing to all other modes in this waveguide. Output modal amplitudes are obtained by mode filtering the three-dimensional output pressure field. We note that the first order approximation of the wide angle PE normal modes [63], which are slightly different from the standard (Helmholtz) normal modes, was used for cleaner mode filtering.

Figure 6-4 presents the results for a wave crossing angle of 40 degrees, which is considered a steep angle. In the top panel, the depth integrated acoustic intensity level is shown referenced to unity $(0 \mathrm{~dB})$ at one meter from the source location. The white dashed lines in all plots denote the centers (crests) of the internal waves. The second plot from the top shows the same depth integrated intensity for an enlarged area of the wave crossings. The lower two plots present the amplitudes of modes one and two (as a reminder, we use a single mode one starting field with unit amplitude at one meter distance from the source in our PE calculations). As one can see, the forty degree crossing angle does not cause any noticeable effects of horizontal refraction. Instead, strong energy coupling to mode two is observed behind the crossing waves. The 

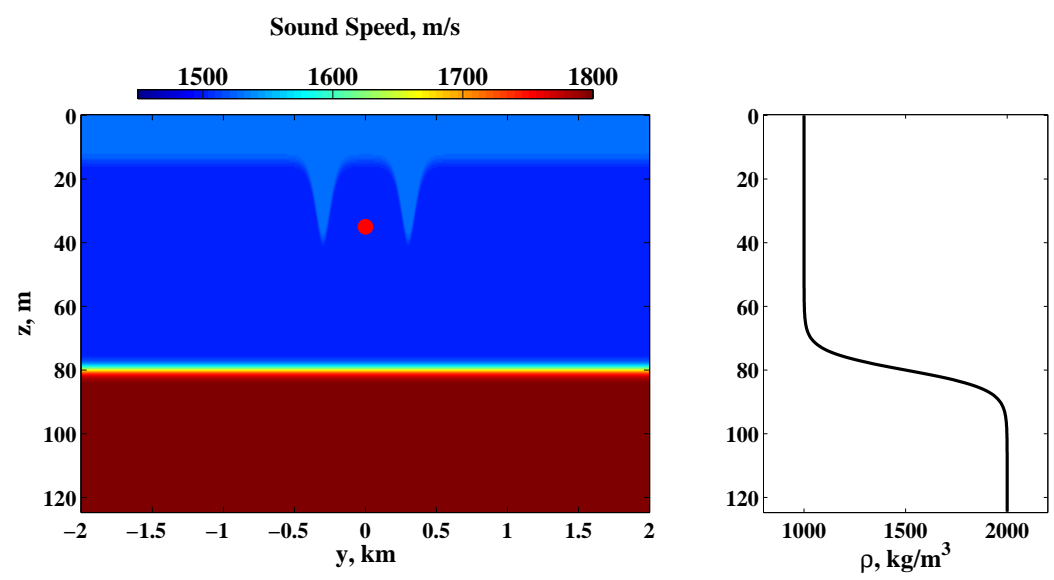

Figure 6-3: Left plot: smoothed sound speed cross-section along $x=0 \mathrm{~m}$. Red circle shows the source location (note that the depth of the source is not specified, since a single mode starting field is used in the program). Right plot: smoothed density column.

strength of this coupling was shown to fluctuate with changing steep crossing angles. Similar to the transparent resonance studied in the previous chapter, the change in the cross angle implies a change in the effective width of the crossing waves that results in the coupled mode intensity fluctuations. We have already mentioned that in real ocean waveguides, internal wave ducts are often seen to terminate after crossing several waves from different trains. Therefore, mode coupled energy remaining in the duct, as shown in Fig. 6-4, is likely to be scattered by further mode coupling and then radiated out at the duct termination. Our numerical modeling of wave crossings at different angles showed no significant changes in physical processes (except for the coupled mode amplitude fluctuations behind the waves) for crossing angles steeper than fifteen degrees.

The case similar to Fig. 6-4, but for waves crossing at an angle of eleven degrees, is shown in Fig. 6-5. At this angle, in addition to a coupled mode energy behind the crossing waves, one can notice that a small amount of mode one energy (and a very small amount of mode two energy) is cross-ducted or escapes from both ducts. The escaped energy behind both ducts is likely to be cross-ducted as well if there are more than two waves in a crossing train. Note that the critical angle of mode one in this example is 5.3 degrees, and the crossing angles at which these effects start 
being noticed are 12-13 degrees, which is approximately twice the critical angle of the incident mode, as predicted. At smaller crossing angles (seven degrees in Fig. 6-6) cross-ducting becomes stronger and we also see significant reflected energy from mode one escaping from the duct. At a crossing angle of seven degrees, the modal intensities of the cross-ducted and reflected acoustic signals are approximately $8 \mathrm{~dB}$ less than the intensity of the signal remaining in the duct. As the angle of crossing becomes even more shallower, cross-ducting and reflection pull a considerable amount of energy out of the initial duct and are as important in magnitude. Figures 6-7 and 6-8 show that after crossing two internal waves at four and two degrees, the depth integrated intensity remaining in the initial duct is $6 \mathrm{~dB}$ weaker than before the crossing, which is considered a significant loss. For comparison, Figs. 6-9 and 6-10 illustrate results similar to Figs. 6-7 and 6-8, but for a single crossing wave (note a single white dashed line indicating the crossing wave crest). Comparing the figures, one sees that although single crossing wave results in a similar modal reflection, it does not provide the effect of cross-ducting, thus pulling out considerably less energy from the initial duct. We also note from Figs. 6-5 - 6-10 that both cross-ducted and reflected energy escaping from the initial duct are nearly adiabatic effects. Similar results were obtained for other incident modes, with the main difference being in the values of the critical angles that determine the cross angle range of observed effects.

We surmise from our numerical modeling that internal wave crossings are responsible for the horizontal refraction and directional spread of modal energy at crossing angles below twice the horizontal critical angles of the normal modes. Together with the known acoustic effects of internal wave curvature [17], shallow water internal waves thus cause uncertainties in the measured angle of signal arrival, which can be more than the 4 degrees observed in the SW06 experiment. In the next chapter, we consider experimental issues and possible methods for measuring the effects of acoustic interaction with crossing internal wave structures. 


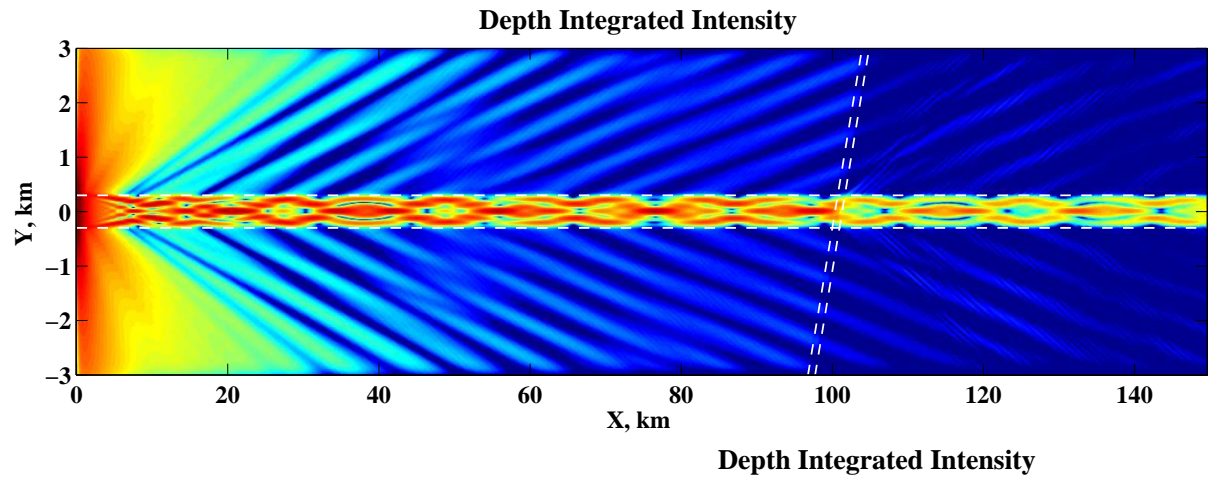

Crossing Angle 40 Deg. dB re 0 source level $\begin{array}{llll}-60 & -50 & -40 & -30\end{array}$
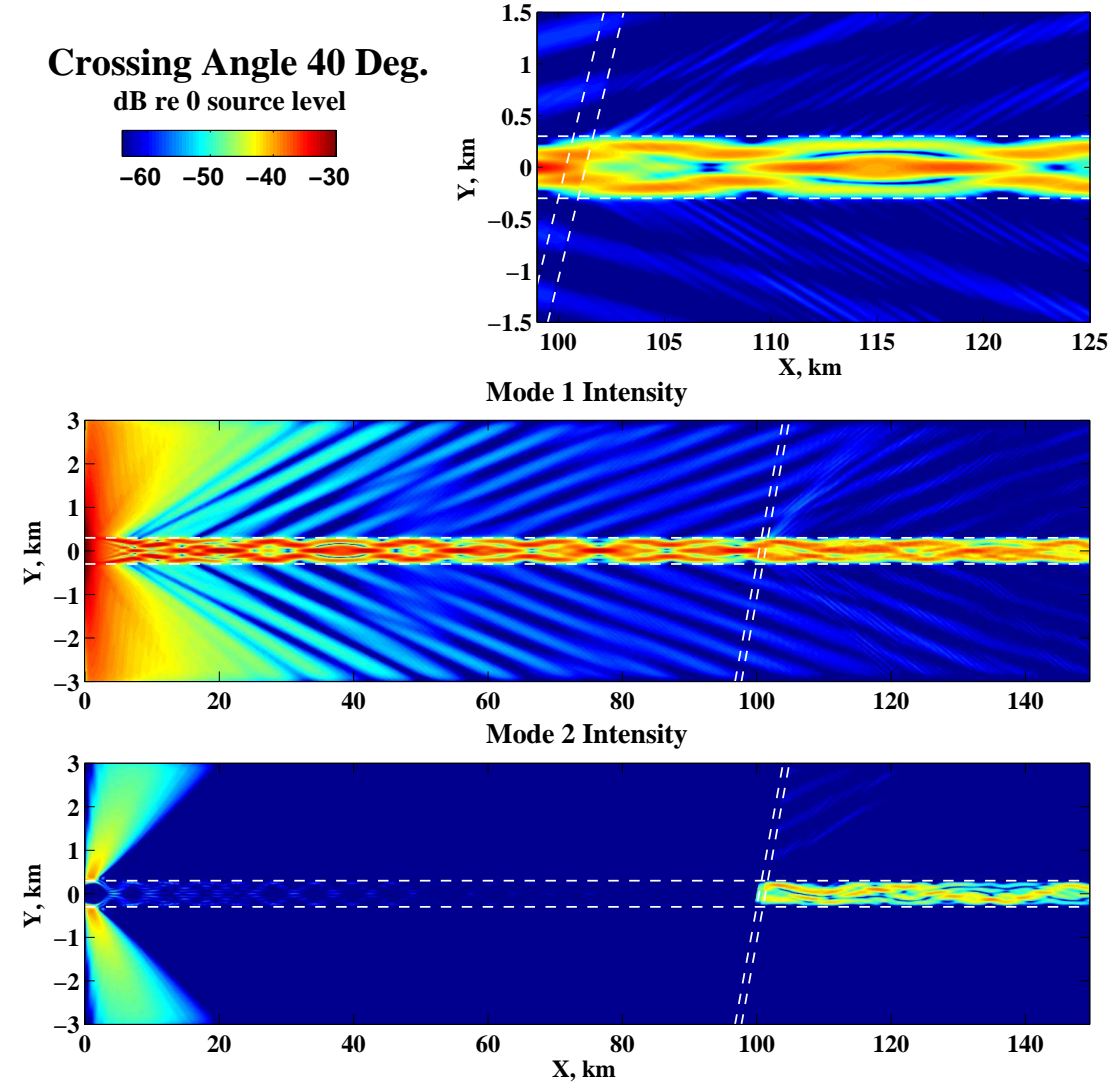

Figure 6-4: Crossing of two identical pairs of internal waves at forty degrees. White dashed lines indicate the wave crests. Depth integrated intensity (re $0 \mathrm{~dB}$ at $1 \mathrm{~m}$ from the source) is shown in the top panel, with an enlarged crossing region in the second panel from the top. Intensities of modes one (initial) and two are shown in the lower two panels. 


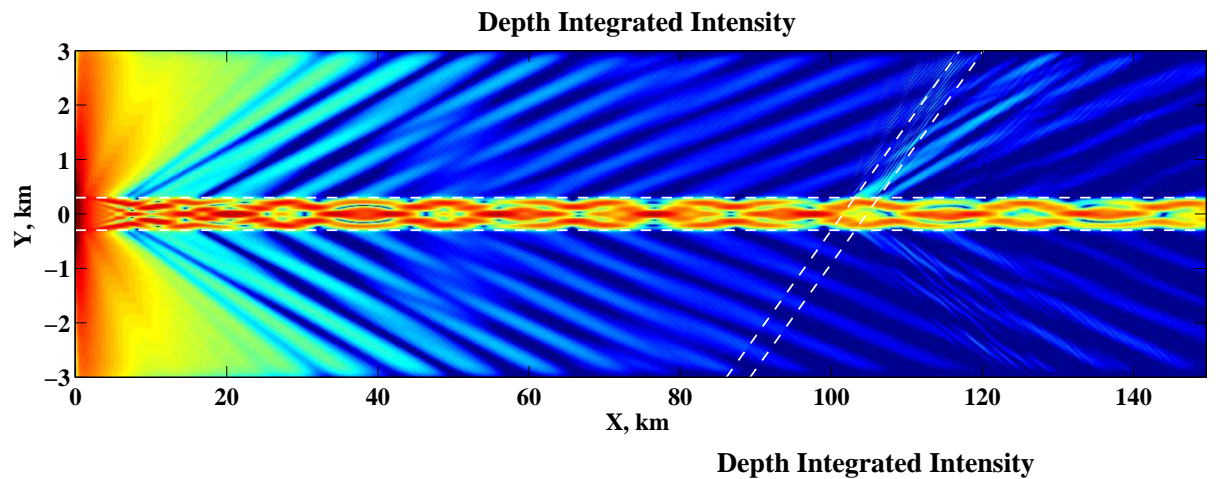

Crossing Angle 11 Deg. dB re 0 source level
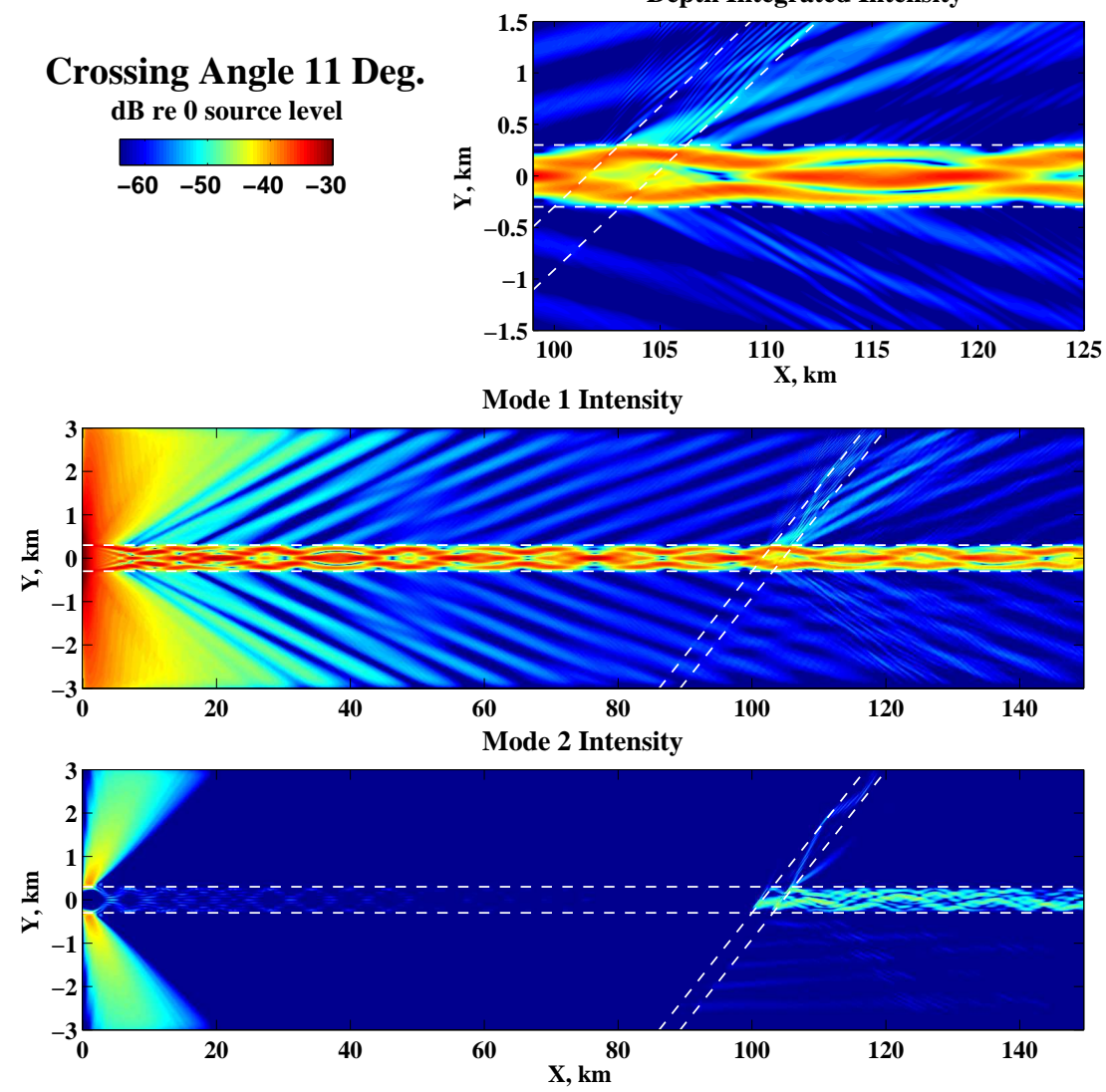

Figure 6-5: Same as in Fig. 6-4, but for a wave crossing angle of eleven degrees. 

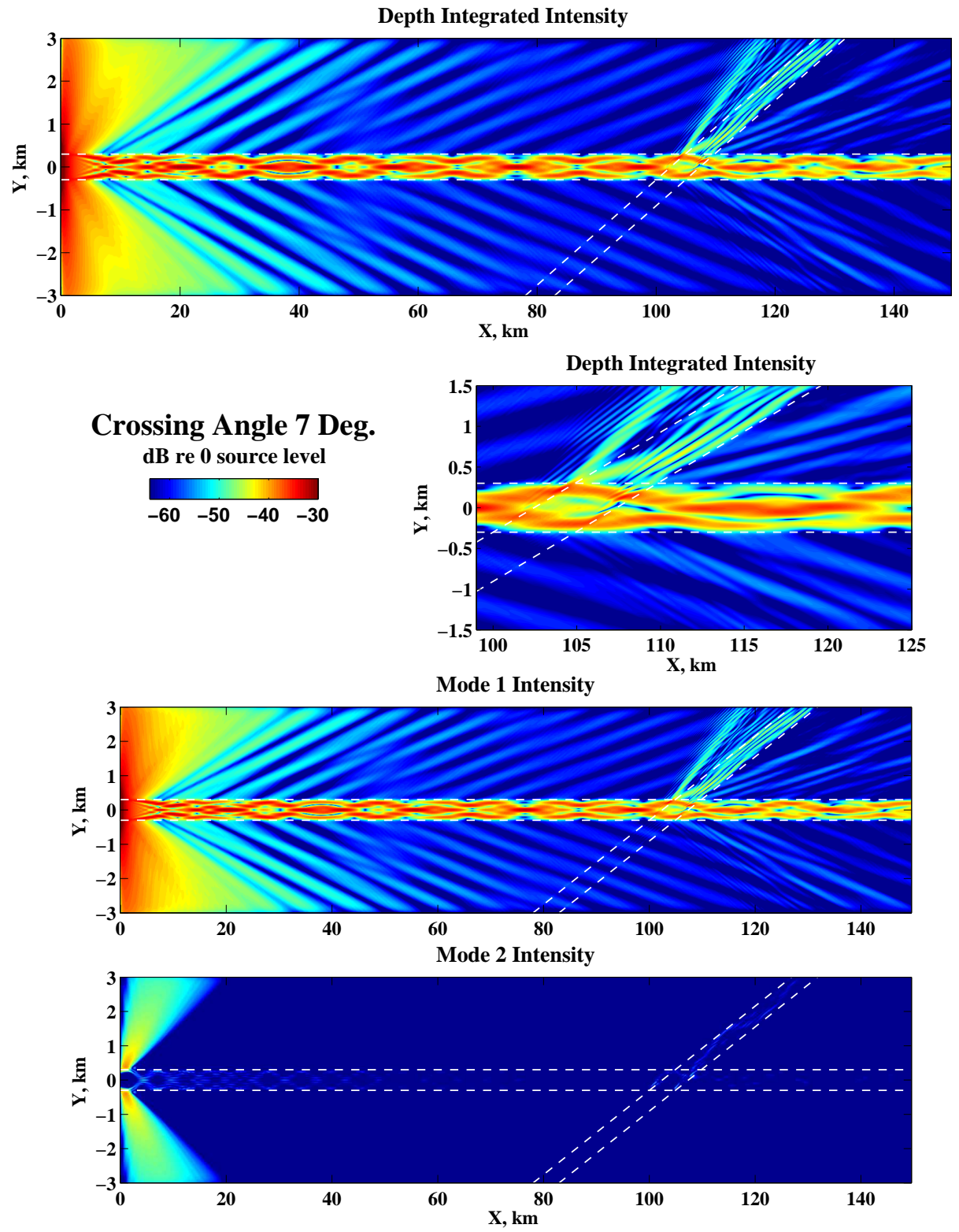

Figure 6-6: Same as in Fig. 6-4, but for a wave crossing angle of seven degrees. 

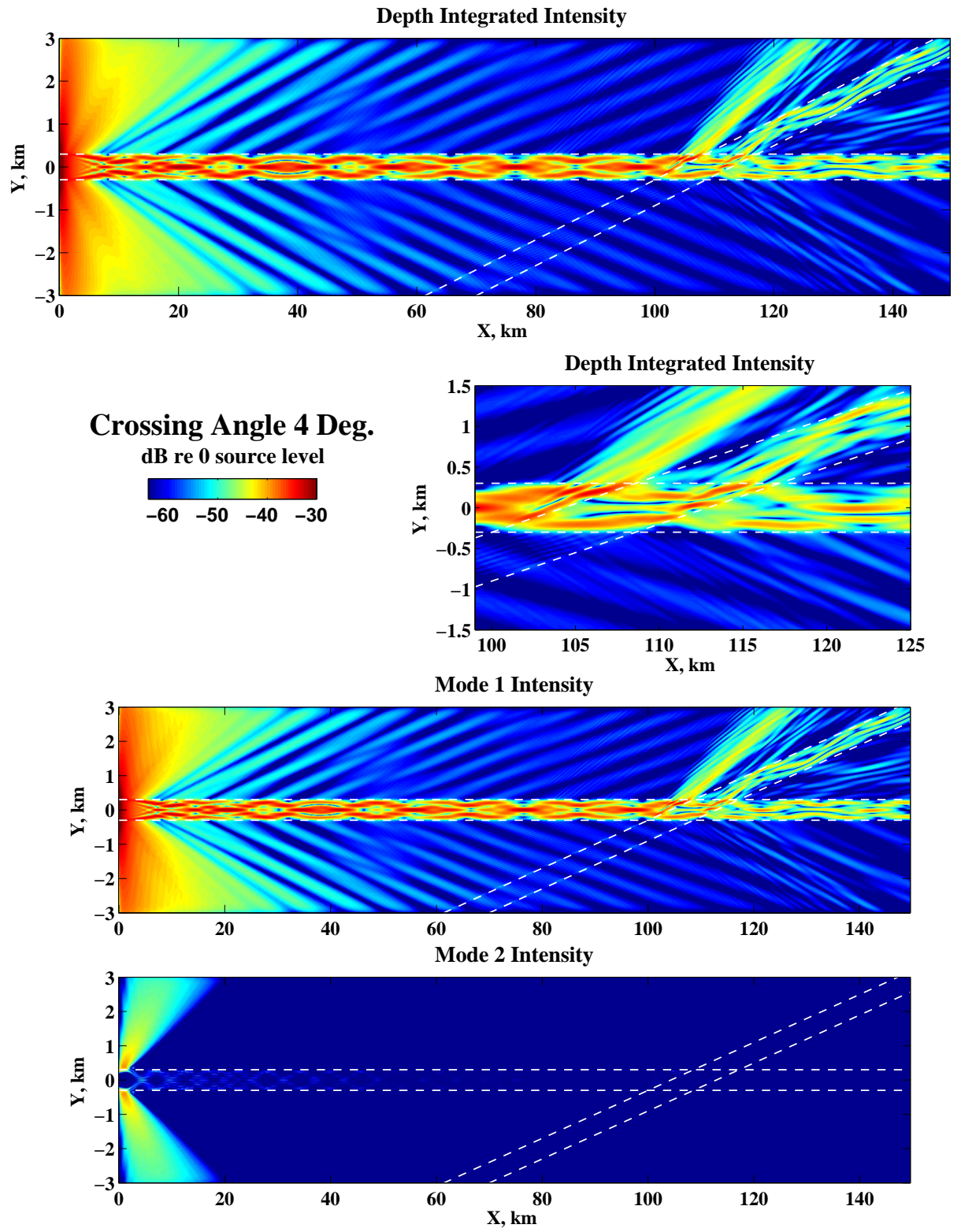

Figure 6-7: Same as in Fig. 6-4, but for a wave crossing angle of four degrees. 

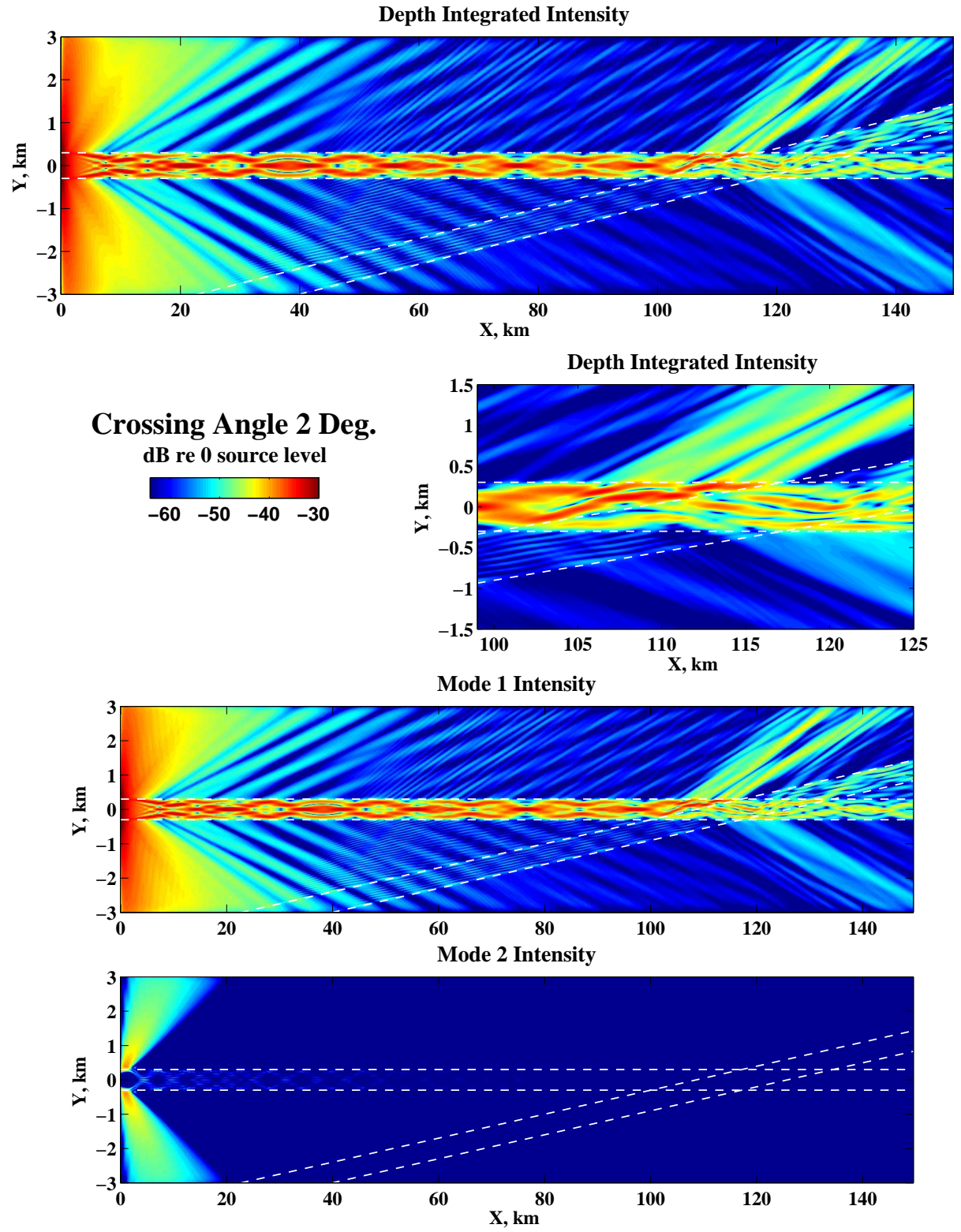

Figure 6-8: Same as in Fig. 6-4, but for a wave crossing angle of two degrees. 


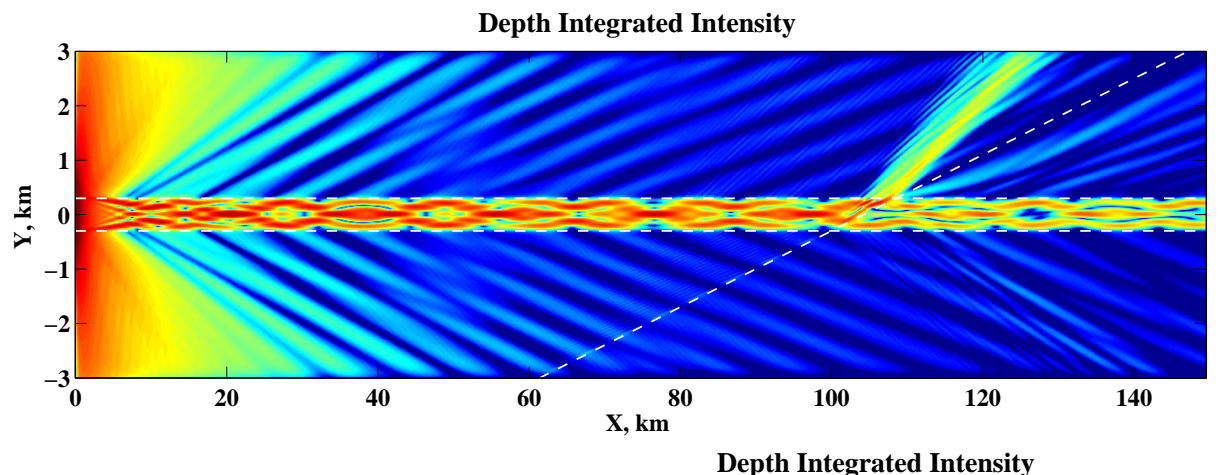

Crossing Angle 4 Deg. dB re 0 source level $\begin{array}{llll}-60 & -50 & -40 & -30\end{array}$
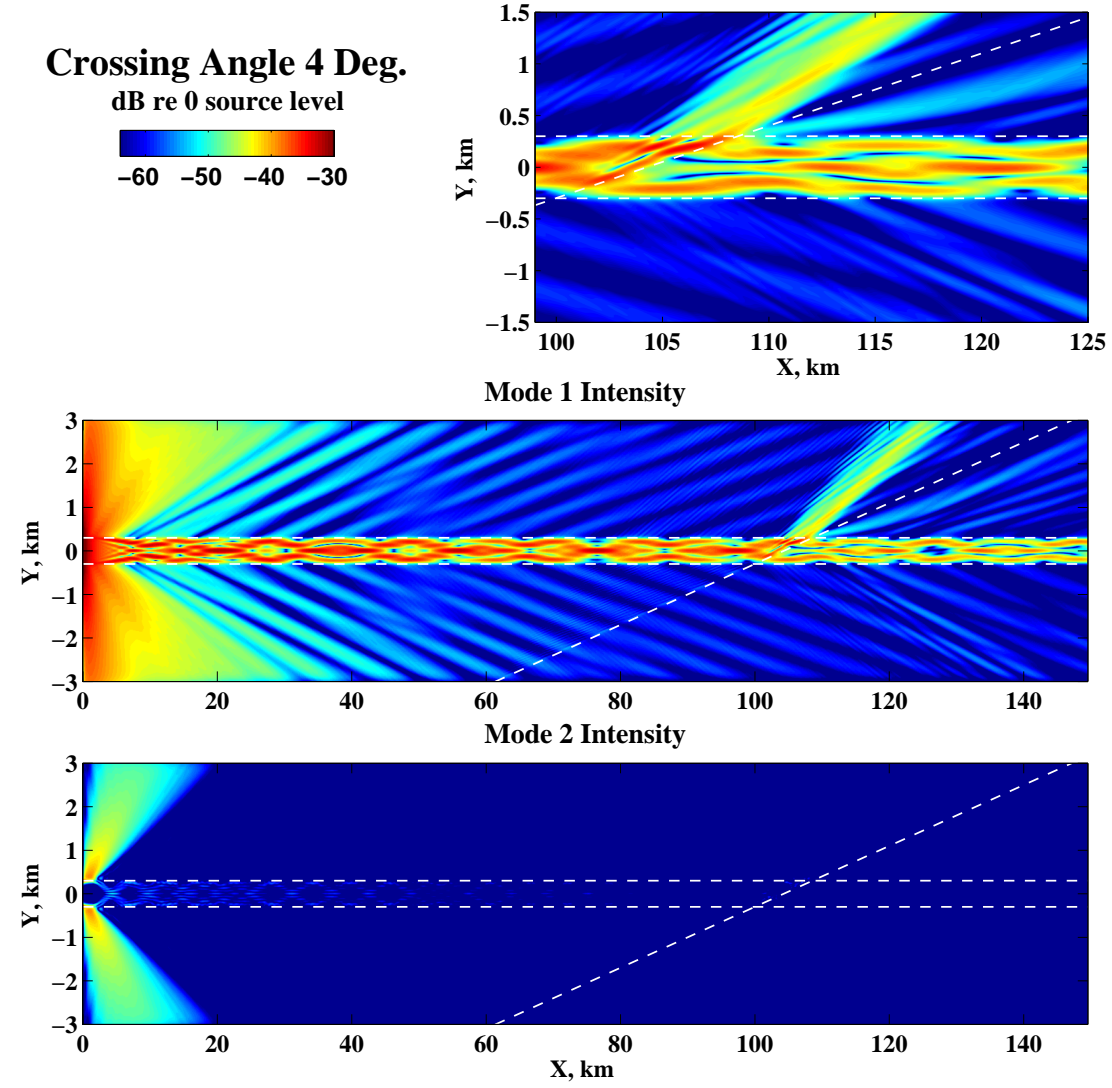

Figure 6-9: Crossing of the parallel internal waves with a single internal wave at four degrees. White dashed lines indicate the wave crests. Depth integrated intensity (re $0 \mathrm{~dB}$ at $1 \mathrm{~m}$ from the source) is shown in the top panel with enlarged crossing region in the second panel from the top. Intensities of modes one (initial) and two are shown in the lower two panels. 

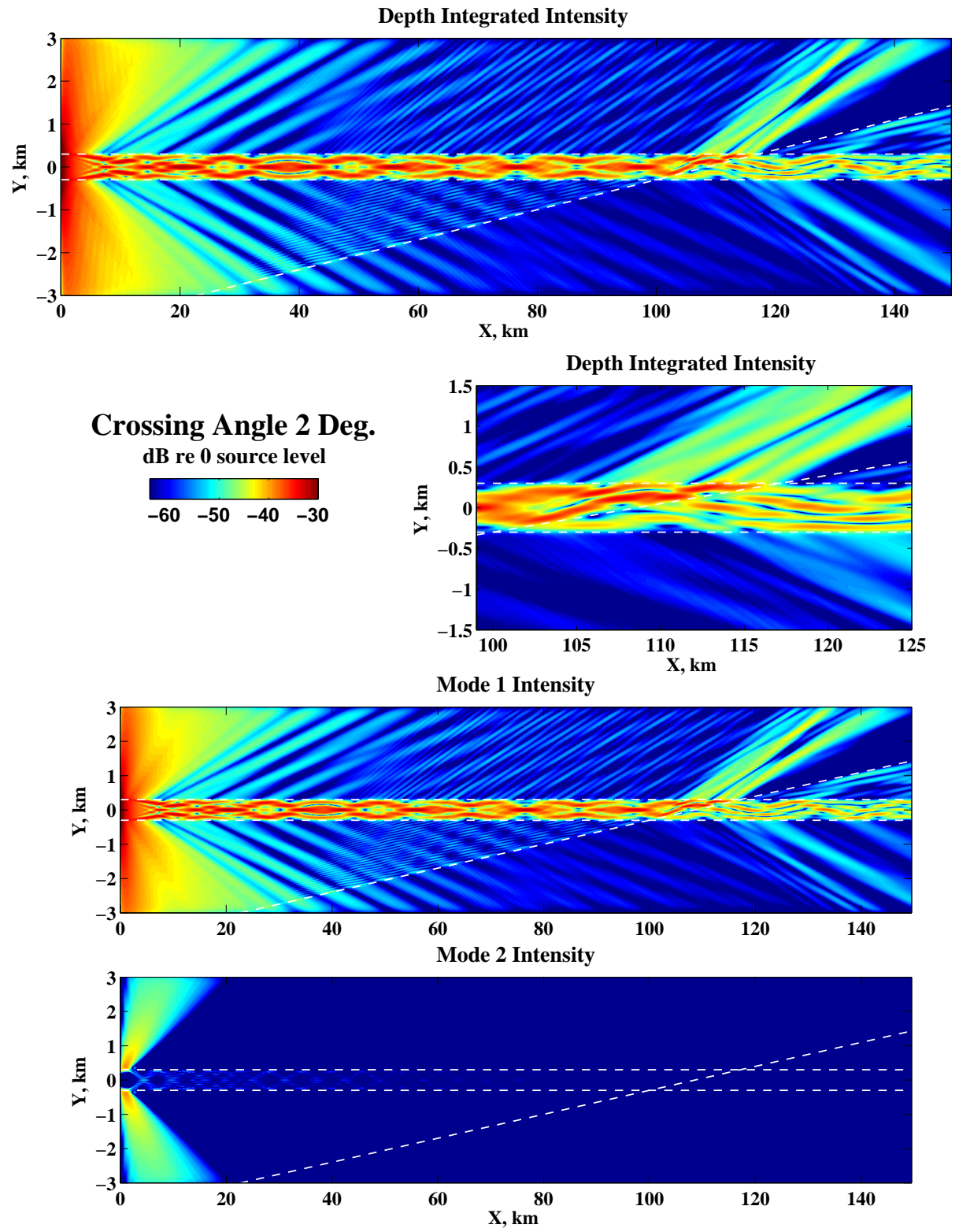

Figure 6-10: Same as in Fig. 6-9, but for a wave crossing angle of two degrees. 


\section{Chapter 7}

\section{Applications to a More Realistic}

\section{Ocean}

In previous chapters we theoretically studied and numerically modeled low frequency acoustic propagation through waveguides that have either straight, parallel waves with infinite crest lengths or crossing structures of parallel waves. We saw that the physics governing the acoustic interaction with all three ocean wave types is similar, with the main difference being in the critical angle distributions across mode number and in mode coupling strengths. In the real ocean, the waves are often not straight. In addition, the amplitudes, shapes, wave crest lengths, and the scale of temporal variability are different for the three wave types. In the following section, we will discuss the existing oceanographic and acoustic data sets and our ideas on possible future experiments that will capture the new effects studied in this thesis in presence of real ocean internal waves. Fully three-dimensional sound interaction with the transverse bedforms (megaripples and sand waves) or with long period surface swells is a new field for acousticians. In this chapter, we will present our (primarily) computational look into a three-dimensional sound wave propagation through waveguides with realistic sea surfaces (Section 7.2) and bottom wave fields (Section 7.3). We will see that both of these oceanographic features can result in a considerable

azimuthal dependence of the acoustic intensity fluctuations. Directions for the future theoretical and numerical studies and our considerations on possible experiments will 
also be outlined.

\subsection{Internal Waves}

Internal waves are common in almost every part of the world ocean. Similar to acoustic waves, the internal wave field can be decomposed into normal modes (and usually, the first one or two modes carry most of the energy) that propagate in the horizontal plane with an associated group speed [64]. Mesoscale variability of the water column properties (eddies, meandering fronts, etc) cause horizontal variability in the buoyancy frequency (Brunt-Vaisala) column that results in the horizontal refraction of the internal wave modes and curvature of its fronts. Bathymetry variability also changes the internal wave modes and eigenvalues. Figure 6-1 shows a SAR image of the nonlinear internal wave activity at the SW06 site taken on July 23, 2006. In this figure, several internal wave trains with noticeable curvature cross each other and compose a complicated pattern. For comparison, a picture in Fig. 7-1 taken on August 8, 2006 shows internal waves with relatively straight line fronts and with only few crossings. As we see, both scenarios can be found in the shallow water.

The statistics of the parameters of crossing internal waves, such as the angle of the crossings, the curvature radius of crossing waves, the distance from the shore of the most frequent crossings, etc. can be studied from analyzing a collection of SAR images and oceanographic data recorded over a long time period. We expect these statistics to be strongly dependent on the geographic location. One of the reasons for this is that the bottom topography that is known to play role in internal wave scattering and generation. Let us consider the interaction of the barotropic tide with the shelfbreak, as schematically shown in Fig. 7-2. If the slope of the continental slope is relatively uniform in the along shelfbreak direction, then the front of the generated internal waves will be essentially plane wave. This would correspond to the relatively straight internal wave scenario (left panel in Fig. 7-2). On the other hand, the shelfbreak and

continental shelf regions that have distinct bottom inhomogeneities such as canyons or shoals can act as strong point sources of the internal waves when interacting with 


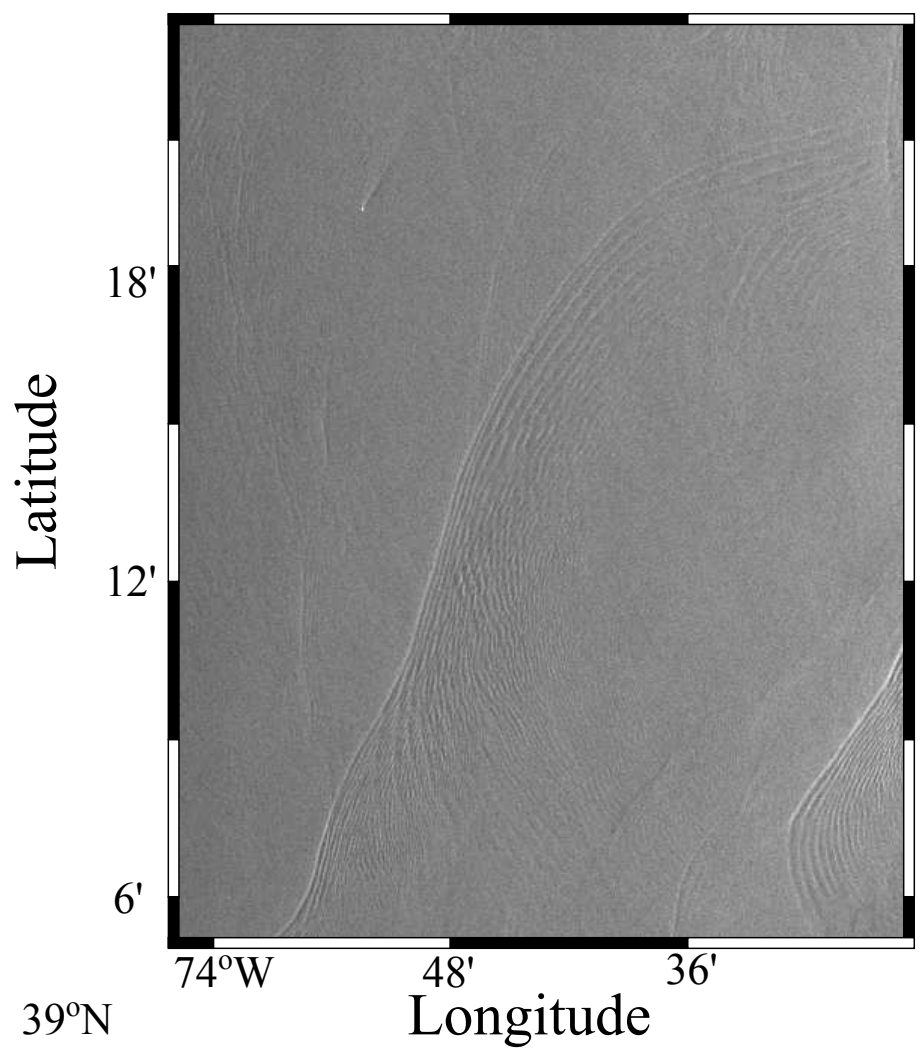

Figure 7-1: SAR image of internal wave activity $20 \mathrm{~km}$ shoreward of the SW06 site taken on August 8, 2006. 

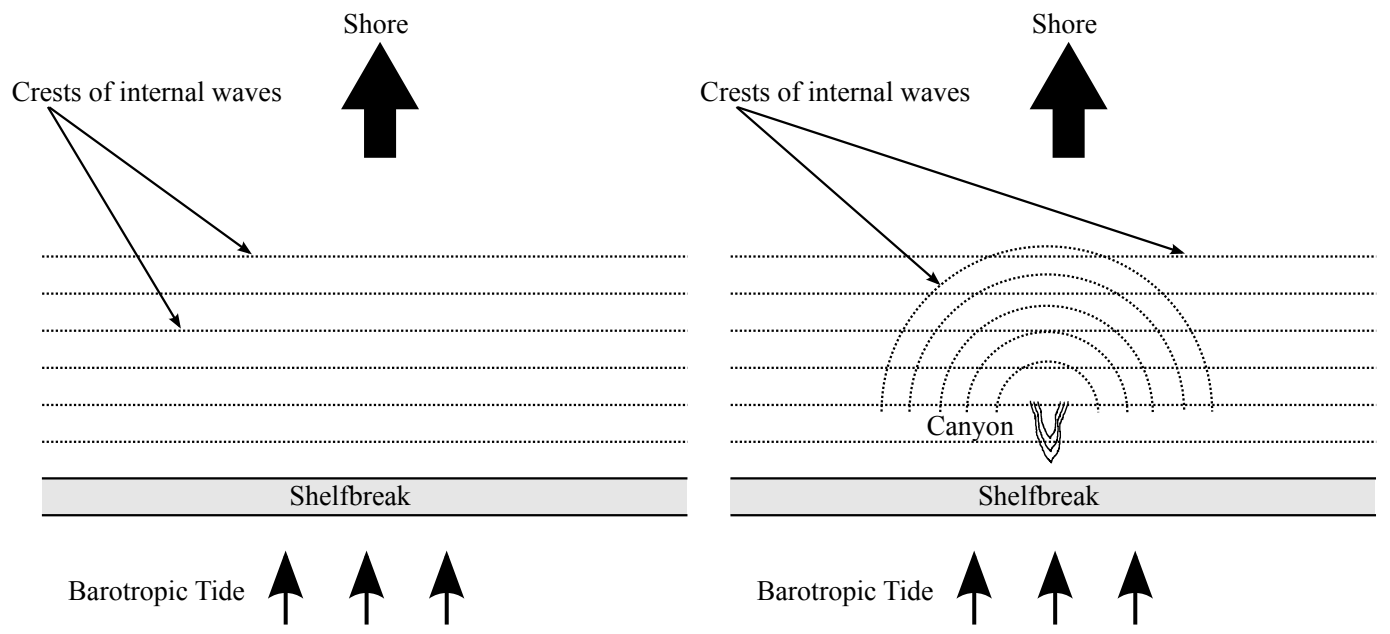

Figure 7-2: Left panel: homogeneous shelfbreak and continental shelf do not affect curvature of the internal wave's fronts. Right panel: interaction of barotropic tides with bottom canyons or shoals results in point sources of internal waves.

the tides (right panel in Fig. 7-2). If one assumes first order cylindrical spreading of the internal wave energy away from these sources, the space statistics of crossing angles and curvatures of the crossing internal waves can be predicted from simple geometric computations. We note however, that the temporal variability of these processes is on the order of several hours and needs to be taken into account if one wants to attempt to observe a specific crossing region.

SW06 was noteworthy for our work in that it contained fully three-dimensional oceanographic and acoustic measurements over two months in an area with intensive internal wave activity. The oceanographic data gathered has high enough spatial and temporal resolution for detailed studies of both the meso- and finer scale oceanography (internal tides and waves). From an acoustic point of view, the fixed source and receiver arrays focused the measurements of sound transmissions to two perpendicular paths: across the internal waves and along the waves. On the along waves acoustic path, strong acoustic horizontal ducting was observed in both the SWARM95 [8] and the SW06 experiments [14]. This geometry also allowed the experimenters to gather the first experimental evidence of the HLM pattern, a new three-dimensional acoustic effect (see Chapter 5) that had not been observed before (Badiey et. al. [15]).

Unfortunately, the SW06 data is not sufficient for experimentally studying the 
effects of secondary ducting or crossing waves, for several reasons. For registering secondary ducting effects, one should locate a source in a primary duct and a receiver array in neighboring ducts that are both relatively straight with no terminations and also have a fixed path of 15-20 km long. Such ducts were not commonly seen, nor was the experiment in this geometry. For crossing waves, if one wants to detect the effects of cross-ducting or the reflected energy escaping from the primary duct, the constraints on the source and receiver location are even more strict. Finally, the schedule of the transmissions in the SW06 experiment was often mismatched with the internal wave activity, making the chance of capturing the right internal wave event together with the proper acoustic transmission small (this was due to marine mammal permitting restrictions, and beyond the experimenters control).

To increase the chances of capturing secondary ducting or crossing waves, several issues need to be considered. First, an experimental area with appropriate bathymetry should be selected according to the discussions above (uniform shelbreak and continental shelf for the secondary ducting and bottom topography with known roughness such as canyons for crossing waves). Second, one could deploy more acoustic receivers several hundred meters apart, as schematically shown in Fig. 7-3, in order to add more along wave acoustic paths with closely spaced directions. This setup will increase chances of capturing the effects of secondary ducting or crossing waves. As an alternative, one could use a fixed receiver array and one or several mobile acoustic sources. This could be a source either towed behind a ship or mounted on an underwater vehicle. In this case, one is able to control the source position and the optimal time of transmission with respect to the moving internal waves. For this case, the internal wave appearance times and the shapes of the wave crests has to be continuously tracked in real time (integrated data from ship based radars with frequently updated SAR images would be a good start). Underwater vehicles with mounted acoustic sources have already been used as a part of the SW06 experiment for studying the anisotropic properties of acoustic transmissions, so this technology exists. 


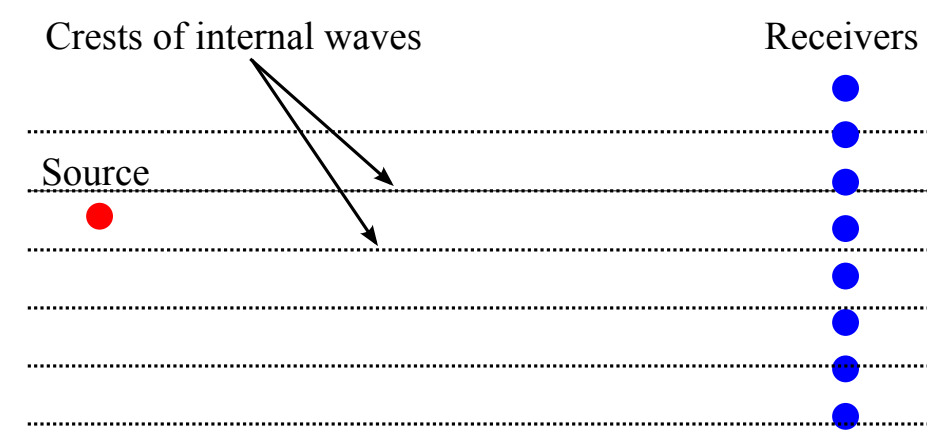

Figure 7-3: Experimental setup with an acoustic source and several closely spaced receivers for increasing the number of closely spaced along wave acoustic paths.

\subsection{Surface Waves}

Ocean surface waves are well known as random scatterers of sound in deep and shallow water ocean waveguides [1]. However, low frequency surface waves (swell) are observed to have a small a decay rate and narrow directional spread [28, 29, 65] at large distances from their origins (storms or hurricanes). Based on our computations for idealized straight waves, we believe long traveling swell can have a noticeable impact on the directional properties of low frequency acoustic field, which have not been studied before. Below we provide a brief description of the directional spectrum of surface waves and our primary computational look into the problem of three-dimensional acoustic propagation through shallow water waveguides that have realistic surface displacements caused by the passing of long period surface swells. We will also present our thoughts on a possible future experiment that will allow one to quantify the proposed directional features of sound scattering in real ocean environments.

\subsubsection{Directional Spectrum}

Ocean surface waves are most often considered as a random process whose statistical properties depend on the sea state [66]. In the fully developed seas, the frequency directional spectrum of the surface waves can be represented as

$$
S(f, \phi)=S(f) G(\phi \mid f),
$$


where the frequency spectrum $S(f)$ is described by the Bretschneider empirical formula $[66]$

$$
S(f)=0.257 H_{1 / 3}^{2} T_{1 / 3}^{-4} f^{-5} \exp \left[-1.03\left(T_{1 / 3} f\right)^{-4}\right]
$$

with frequency $f$ given in Hertz, significant waveheight $H_{1 / 3}$ given in meters and significant period $T_{1 / 3}$ given in seconds. There exist a number of similar forms describing the directional function $G(\phi \mid f)$, and the one we will use here was proposed by Mitsuyasu et. al. [67] based on experimental observations. It is

$$
G(\phi \mid f)=G_{0} \cos ^{2 s}\left(\frac{\phi-\phi_{0}}{2}\right)
$$

where

$$
s= \begin{cases}\left(f / f_{p}\right)^{5} s_{\max }, & f \leq f_{p}, \\ \left(f / f_{p}\right)^{-2.5} s_{\max }, & f \geq f_{p},\end{cases}
$$

$f_{p}=1 /\left(1.05 T_{1 / 3}\right)$ is the frequency of the spectral peak, $s_{\max }$ is the parameter defining the directional spread and the constant $G_{0}$ is

$$
G_{0}=\frac{1}{\pi} 2^{2 s-1} \frac{\Gamma^{2}(s+1)}{\Gamma(2 s+1)},
$$

where $\Gamma(\cdot)$ denotes the Gamma function. Computational analysis by Goda et. al. [68], that also agrees with the directional spread observations for the swell traveling across the Indian ocean by Ewans [65], suggests that $s_{\max }=75$ is a reasonable value for the spread of swell waves with a long decay distance. As an example, Fig. 7-4 shows the directional spectrum of the swells with the following parameters: $H_{1 / 3}=2$ $\mathrm{m}, T_{1 / 3}=17 \mathrm{~s}, s_{\max }=75, \phi_{0}=0 \mathrm{deg}$. These parameters are close to observed values for the long traveled swell [29]). Figure 7-5 illustrates one realization of the sea surface elevation, together with an enlarged view, based on this spectrum. 


\subsubsection{Numerical Modeling}

The ocean swell field has the most noticeable temporal variability as compared to the other two types of waves. Its characteristic phase speeds vary from a few to tens of $\mathrm{m} / \mathrm{s}$. For acoustic frequencies considered in the present work (less than $500 \mathrm{~Hz}$ ), this will result in a few $\mathrm{Hz}$ of the Doppler shift. Such correction is not considered important for the governing physics of the three-dimensional acoustic effects studied above. Therefore, in our acoustic computations we will use the frozen waveguide approximation, i.e. the waveguide properties are assumed to be stationary over the time of acoustic propagation. As a background waveguide, we use the two layer and half space waveguide model described in Chapter 4 with the same parameters $\left(c_{0}=\right.$ $1500 \mathrm{~m} / \mathrm{s}, c_{m l}=1530 \mathrm{~m} / \mathrm{s}, c_{\text {bot }}=1800 \mathrm{~m} / \mathrm{s}, \rho_{0}=1000 \mathrm{~kg} / \mathrm{m}^{3}, \rho_{\text {bot }}=2000 \mathrm{~kg} / \mathrm{m}^{3}, H_{\text {bot }}$ $=80 \mathrm{~m}, D=15 \mathrm{~m})$. Swell is incorporated into this model as the sea surface elevation shown in Fig. 7-5. Unfortunately, currently available three-dimensional parabolic equation programs are not capable of handling the given sea surface elevation and we were not thus able to provide a fully three-dimensional coupled mode solution. Instead, we compute the phase speed of each normal mode with the KRAKEN [32] normal mode program mode across the domain and use the RAM parabolic equation

program [69] to simulate horizontal adiabatic propagation separately for each mode. Possible effects of mode coupling will be discussed later in this section.

By placing the acoustic source at $\left(x_{s}, y_{s}\right)=(0,0) \mathrm{m}$ and assuming the amplitude of each mode to be $0 \mathrm{~dB}$ at one meter away from the source, four horizontal propagation simulations, one for each of the directions of $x$ - and $y$-axes, were performed for each normal mode. Total solution for each normal mode was then combined by stitching four separate quadrant solutions along the forty five degree bearings $x=y$ and $x=-y$.

Figure 7-6 shows resultant amplitudes of modes 1, 3, 5 and 7 for the source frequency of $100 \mathrm{~Hz}$. One can see from this figure that, in the absence of mode coupling, mode 1 propagation has very small or no azimuthal dependence. On the other hand, plots for mode 3,5 and 7 show scintillations of their amplitudes within a 


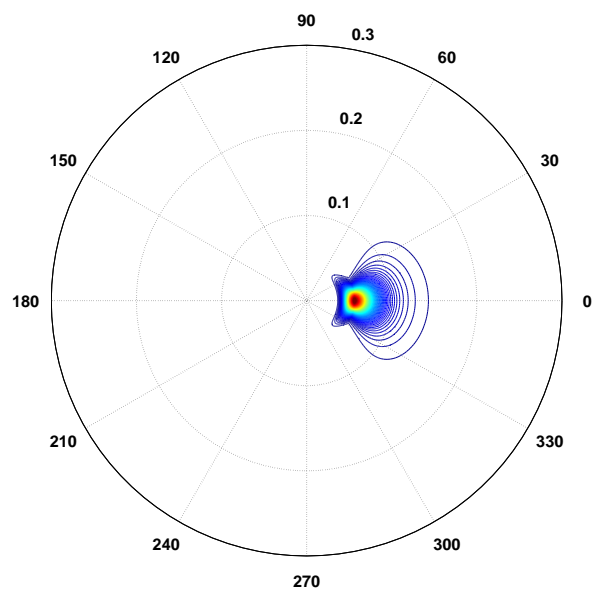

(a)

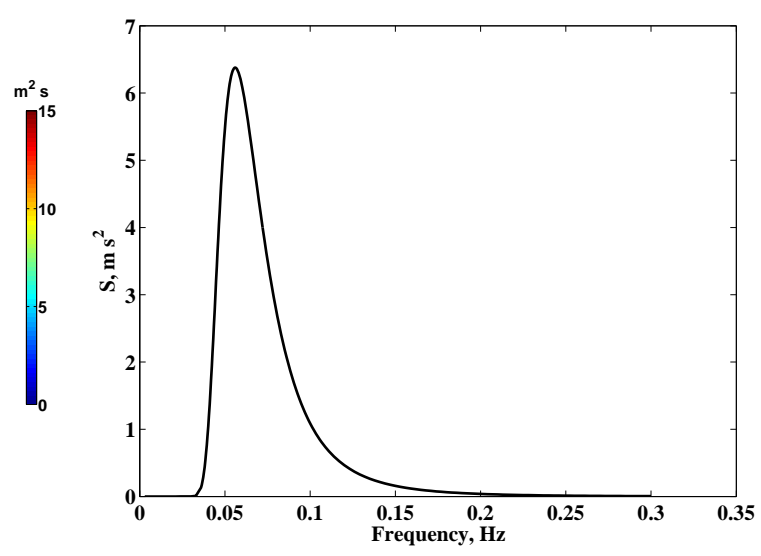

(b)

Figure 7-4: (a) Directional spectrum for surface swell with $H_{1 / 3}=2 \mathrm{~m}, T_{1 / 3}=17$ $\mathrm{s}, s_{\max }=75$ and $\phi_{0}=0$ deg. (b) Directional spectrum shown in (a) plotted for the dominant azimuth $\phi_{0}$.

certain azimuthal range centered at the dominant direction of the ocean wave crests $(x=0)$. The amplitude of these scintillations, as well as their azimuthal range, increases with mode number (note the very strong scintillation of more than $20 \mathrm{~dB}$ for azimuthal range of $90 \mathrm{deg}$ of mode 7 amplitude). This mode number dependence is consistent with the horizontal critical angle analysis studied in Chapter 5: mode one has very small critical angle because of its insensitivity to the sea surface disturbance, whereas higher order modes span the whole water column and are therefore affected by the local horizontal ducts in between surface swell troughs.

Another interesting feature that can be seen from the plots in Fig. 7-6 is the range dependence of the amplitude scintillations. Narrow beams of increased modal energy are noticed to be more distinct at the shorter distances from the source (modes 5 and 7 are explicit examples). These beams are likely to result from the normal mode interaction with the closest local surface wave ducts to the source. At longer ranges (more than approximately $5 \mathrm{~km}$ ), further interaction of the normal modes with the surface waves diffuses its energy into other short local ducts, smearing the initial azimuthal pattern. This is an interesting phenomenon suggesting existence of the certain distance from the source at which scintillations of modal amplitudes will be the most noticeable. This is consistent with the analysis of the range dependent 


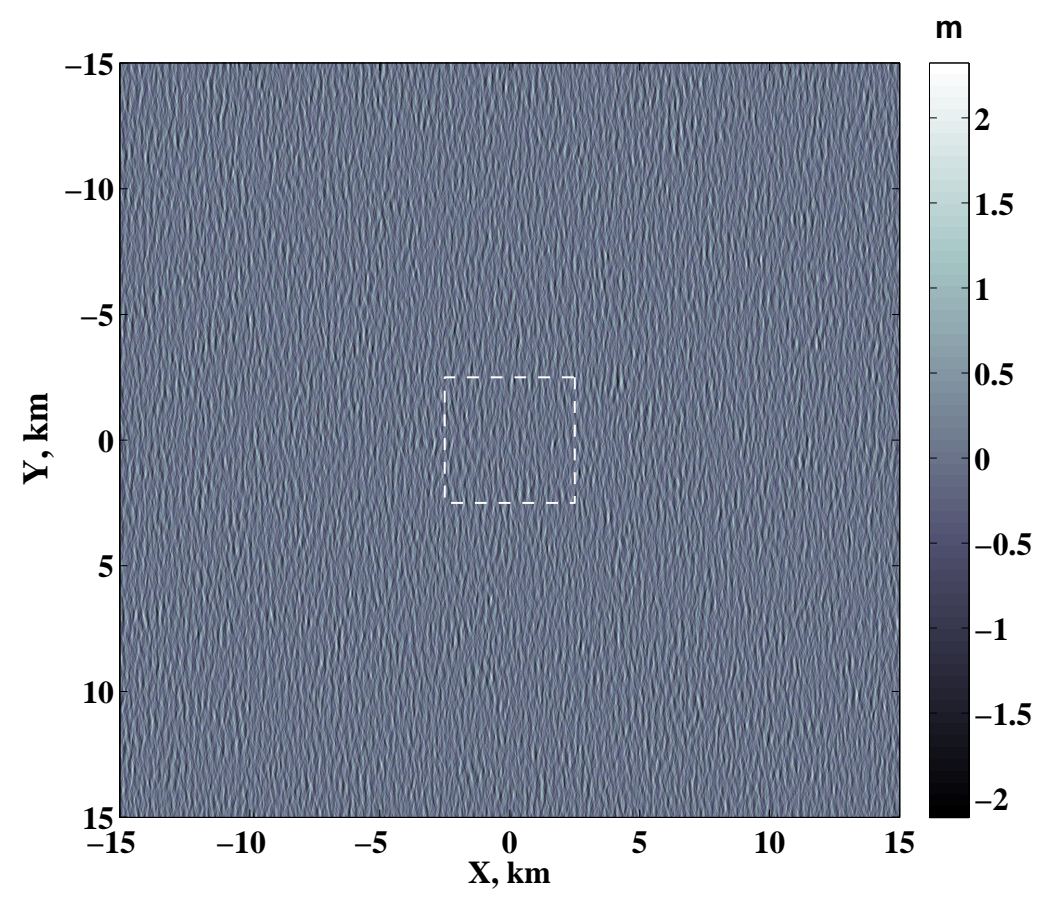

(a)

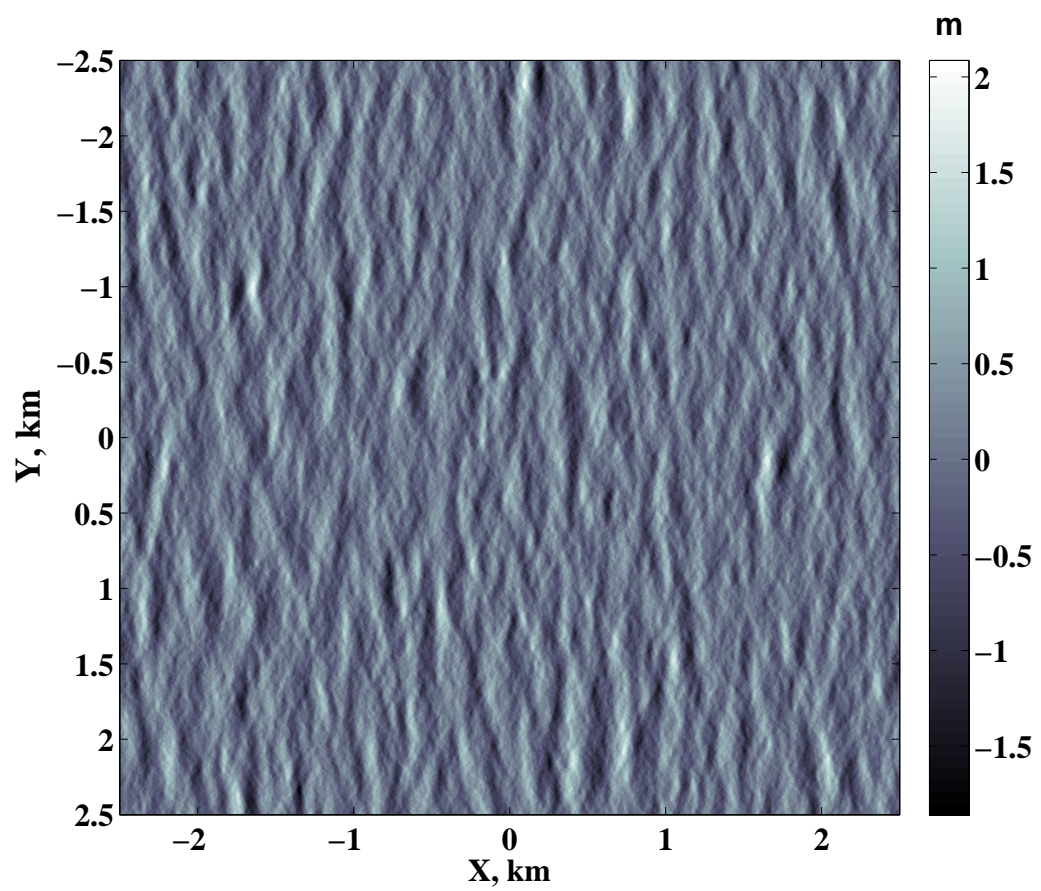

(b)

Figure 7-5: (a) Realization of the sea surface elevation based on directional spectrum shown in Fig. 7-4. (b) Enlarged view of (a) shown for the region inside the dashed line. 

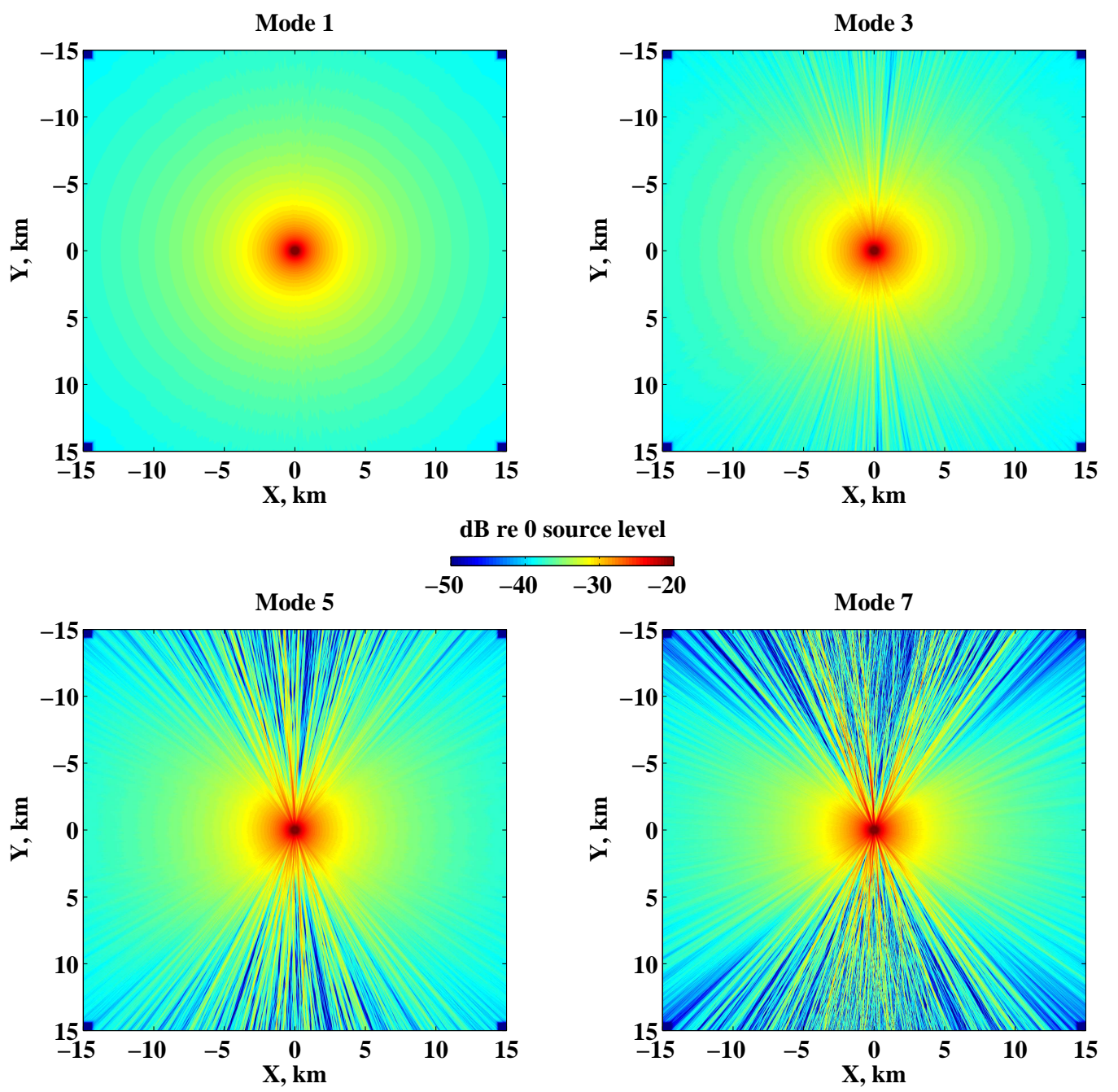

Figure 7-6: Amplitudes of modes 1, 3, 5 and 7 for adiabatic propagation from the source, which is located at $\left(x_{s}, y_{s}\right)=(0,0) \mathrm{m}$, and has frequency $100 \mathrm{~Hz}$. 
intensity scintillation caused by internal wave scattering [70].

\subsubsection{Mode coupling}

We have seen from our numerical modeling that adiabatic normal mode propagation suggests significant amplitude scintillations in the wide range of azimuths (up to $90 \mathrm{deg})$ for higher order modes. Here we address the next obvious question: "Is the adiabatic approximation valid for these applications to surface swells"? To answer this question, one should evaluate the strength of mode coupling effects in the across wave crest directions, which correspond to the most rapid changes in modal properties. We return to the sharp interface approximation (SIA) of surface waves used in Chapter 5 to explore the feature of mode coupling in these applications. Consider an example of the plane wave normal mode boundary problem (similar to Section 5.3.1) at a sharp interface dividing the unperturbed background waveguide and its perturbation due to a surface depression (Fig. 7-7a). Here we limit our discussion to the normal incidence of initial mode $n$. As an example, Fig. 7-7b represents the normal mode solution due to incident mode 5 with unit amplitude at frequency $100 \mathrm{~Hz}$ with surface depression of $2 \mathrm{~m}$. The black line shows the vertical structure of the solution for the total pressure field along the interface on the unperturbed side of the interface, and the blue line shows the corresponding solution for the perturbed side. The red line represents the difference between the solutions on both sides. As we see, neglecting the continuous spectrum results in a slight mismatch between solutions on both sides of the sharp interface. In addition, the difference between solutions resembles the shape of mode 8, which is not a propagating mode in either the perturbed or unperturbed waveguides. This implies a leakage of modal energy into the continuous spectrum, or equivalently, into the seabed. Mode coupling within discrete spectrum for this waveguide is shown in Fig. $7-8$ by the coupled mode reflection and transmission coefficients (defined in Section 5.3.1) at normal incidence upon the sharp interface. Similarly to internal waves and bottom waves, the coupled reflection is reasonably small for all incident modes. The transmission coefficient shows the strongest coupling between higher order neighbor modes. We note, however, that the strength of the mode coupling 


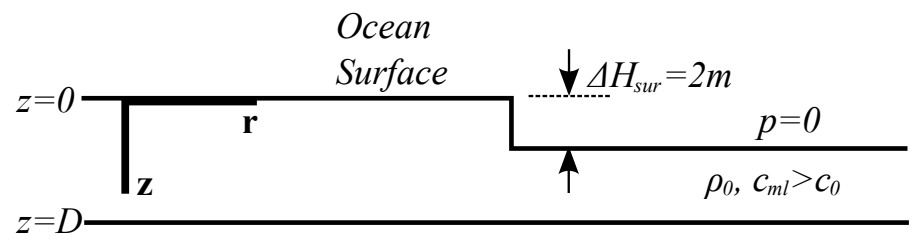

$\rho_{0}, c_{0}$



(a)

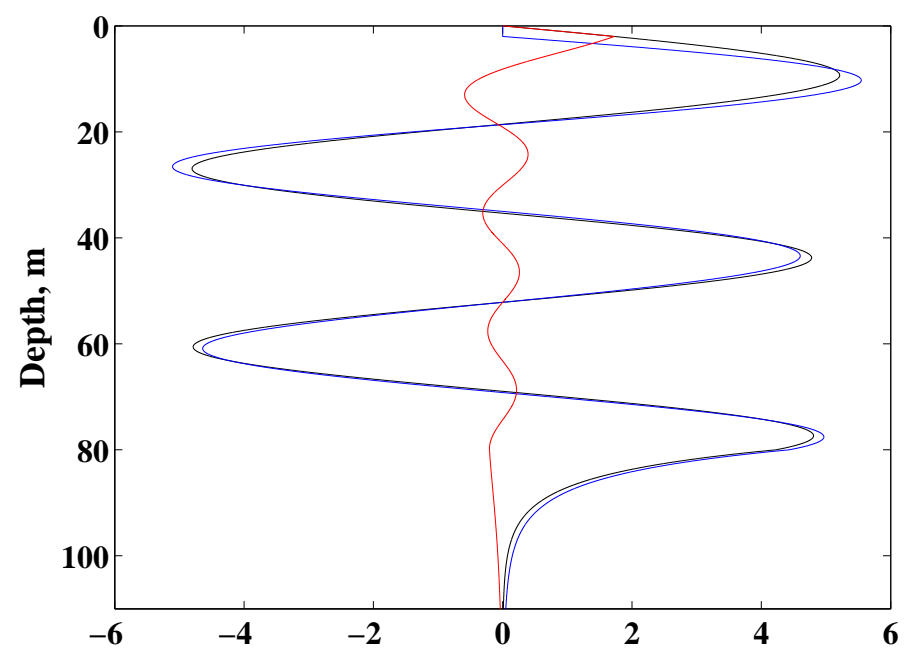

(b)

Figure 7-7: (a) Vertical sharp interface dividing unperturbed waveguide and its perturbation due to surface depression of amplitude $2 \mathrm{~m}$. (b) Pressure field solution at the unperturbed side of sharp interface (black) and at the unperturbed side (blue). Red line represents the difference in solutions on both sides. 


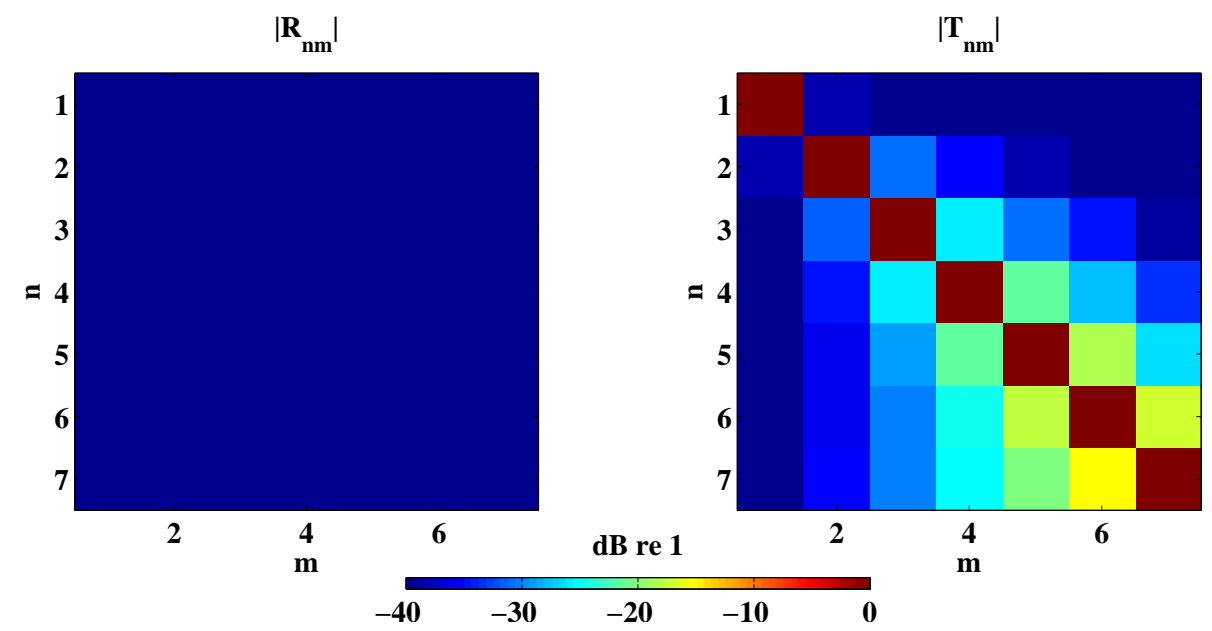

Figure 7-8: Plane wave mode coupled reflection (left plot) and transmission (right plot) coefficients for the normal incidence upon the sharp interface between unperturbed waveguide and its perturbation due to surface depression of amplitude $2 \mathrm{~m}$ and frequency $100 \mathrm{~Hz}$.

into the continuous spectrum is larger than into propagating modes, and therefore the overall loss of modal energy dominates the coupling between propagating modes. Note that 90 deg is the worst case and that at shallower angles, coupling strength is much less.

\subsubsection{Practical Considerations}

It was shown above that low frequency acoustic propagation in the presence of long traveling surface swells can result in considerable fluctuations of the higher order mode amplitudes within a broad (up to $90 \mathrm{deg}$ ) azimuthal range centered at the dominant direction of wave crests. We also noted that the energy leakage of higher order modes into seabed dominates the coupling between propagating modes. As a result, one would expect to observe modal fluctuations within a sector bounded in both azimuth and range from the source. Lynch et. al. showed a considerable (6 dB) azimuthal fluctuations of the transmission loss using mobile sources as a part of the SW06 experiment [16]. According to their results, a number of possible factors could create such anisotropy, including a space-time undersampling, uncertainty in the tracking positions and three-dimensional impact of the fine-scale oceanography. 
We note that in addition to the strong nonlinear internal wave activity in the SW06 site, surface swells that were possibly passing through the SW06 site (note that during the experiment, there were two storms with the peak wind speeds of $20 \mathrm{~m} / \mathrm{s}$ ), could also contribute to the above mentioned results.

It would be of great interest to experimentally measure the sound intensity scintillations and its modal components solely due to the surface swells. For doing that, a simple and cost effective experiment could be provided using a single moored vertical line hydrophone and thermistor array (VLA) and one mobile source (small AUV with mounted acoustic source is an excellent solution) with available tracking equipment. By programming the source to continuously transmit the signal and circle around the VLA with slowly increasing radius, one is able to gather a bulk of data necessary to experimentally study this effect.

\subsection{Bottom Waves}

Transverse seabed patterns in the forms of megaripples or large and very large sand waves are commonly observed $[23,24]$ on the continental shelves and slopes. In fact, some ocean regions [25] are densely populated with these types of entities. Although the oceanographic properties and origins of these bedforms have been studied for decades now [22], their three-dimensional implications for low and medium frequency acoustics have not received particular attention. Moreover, fine scale bathymetry is often lowpass filtered out in computational acoustics. Unlike surface swell and internal waves, waves of this type are not moving. However, they are often characterized by asymmetric shape and irregular seabed properties across the wave [57]. This can result in asymmetry of the acoustic properties across the wave. Such irregularities will not change the horizontal critical angle of normal modes, but can result in the asymmetric mode coupling in the across wave directions. In this section, we will

provide computational results for three-dimensional acoustic propagation through waveguides with densely populated sand waves, using real high resolution bathymetry data. 
Giant sand waves with the crest to crest length exceeding $200 \mathrm{~m}$ and amplitudes greater than $10 \mathrm{~m}$ have been observed at the mouth of San Francisco Bay in 2005 and described in [71]. High resolution $(2 \times 2 \mathrm{~m})$ bathymetry data was gathered with multiple multibeam sonar measurements and is available at the Seafloor Mapping Lab electronic database at [72]. Figure 7-9 shows a three-dimensional colored view of the bay mouth bottom relief. A two-by-two kilometer domain in the center of the mouth (area shown inside by the dashed line) shows very regular sand waves on the seabed, with mean depth ranging from 30 to $106 \mathrm{~m}$. In Chapter 5, we noticed that in an 80 m deep waveguide, straight and parallel bottom waves of only one meter amplitude result in horizontal reflection and ducting of the sound wave energy within a five degree range of the horizontal grazing angles. Our estimates for the San Francisco Bay suggest horizontal critical angles exceeding twenty degrees! Without a doubt, straight bottom waves of such amplitude on a flat seabed will be very strong sound reflectors. Our main goal here is to explore the effects of the natural ducts with noticeable curvature, varying strength and rapidly changing water depth on acoustic propagation properties.

For our numerical modeling, we decided to to use the constant sound speed of 1500 $\mathrm{m} / \mathrm{s}$ in the entire water column, and so the variability of the sound speed column is assumed to have a second order effect on acoustic waveguide properties compared to the large variation of the bottom depth. Using the three-dimensional wide angle parabolic equation program used in the previous chapter, we simulate the full field propagation of a continuous wave acoustic signal from a point source located at $\left(z_{s}, y_{s}, z_{s}\right)=(0,0,10) \mathrm{m}$ with frequency $500 \mathrm{~Hz}$ across the selected domain shown by a dashed line in Fig. 7-9. Top panel of Fig. 7-10 shows the bathymetry of the selected region in the Cartesian coordinates of computational domain. For illustrative purposes, bottom depth along transect $x=1000 \mathrm{~m}$ (dashed line in the upper plot) is shown in the bottom panel of Fig. 7-10.

Figure 7-11 shows depth integral of the computed acoustic intensity. A first look at this figure shows a very good correlation with bathymetry data. Similar to results for the adiabatic propagation of high order modes in the presence of surface swell as 
described in the previous section, we clearly see narrow beams of increased acoustic intensity originating close to the source location. However, unlike the case of surface waves, we see that the intensity of the resultant pressure field that contains all the normal modes has fluctuations of $8 \mathrm{~dB}$ at one kilometer distance from the source. We also see that some of these beams follow the lines of the sand wave crests with a strong curvature with radius of approximately $2 \mathrm{~km}$. Horizontal ducting in between curved internal waves was studied theoretically and observed numerically before [17], but for the curvatures with ten times greater radius.

The striking results of our numerical modeling clearly show the significance of the natural transverse bedform fields in three-dimensional ocean acoustics. It also piques our interest for a possible small scale experiment in this bay for a quantitative validation of the numerical results. As we mentioned in the previous section, a small AUV with a mounted acoustic source and a single mooring is an efficient way of measuring directional properties in an anisotropic waveguides. However, if one attempts to launch an unmanned vehicle in the mouth of San Francisco Bay, special care should be taken due to the strong currents with speed exceeding five knots (which is comparable to the speed performance of most available AUVs), and high shipping activity. As a more efficient and very cheap alternative, a single hydrophone mooring can be deployed at the optimal location for recording shipping noise. If a visual tracking of the passing by ships is established (two high resolution camcorders mounted on shore at the optimal look angles), it should be possible to measure the directional properties of the received intensity. 


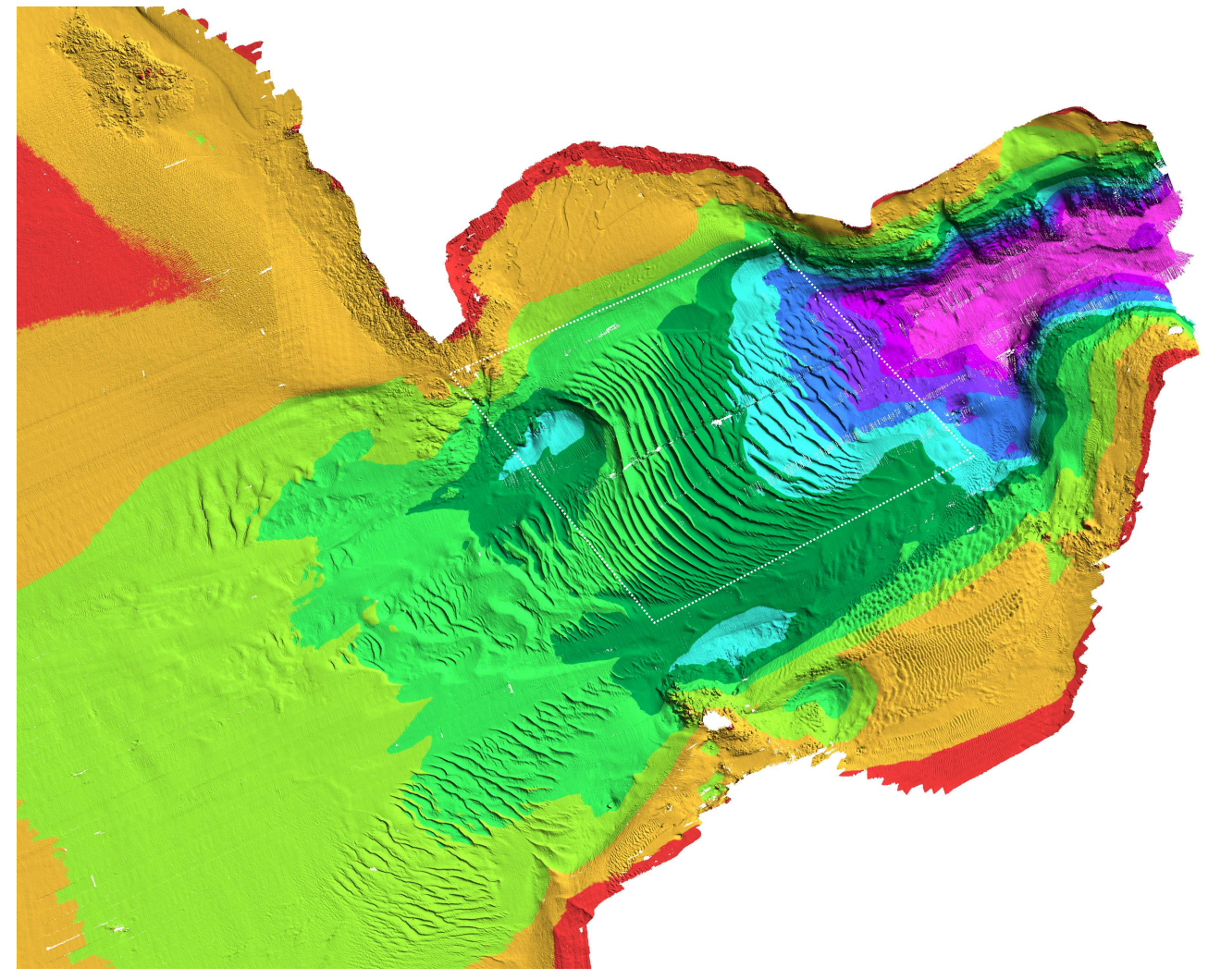

Figure 7-9: Three-dimensional view of the bottom relief at the mouth of San Francisco Bay. Dashed line shows the domain selected for the three-dimensional acoustic propagation numerical modeling. 


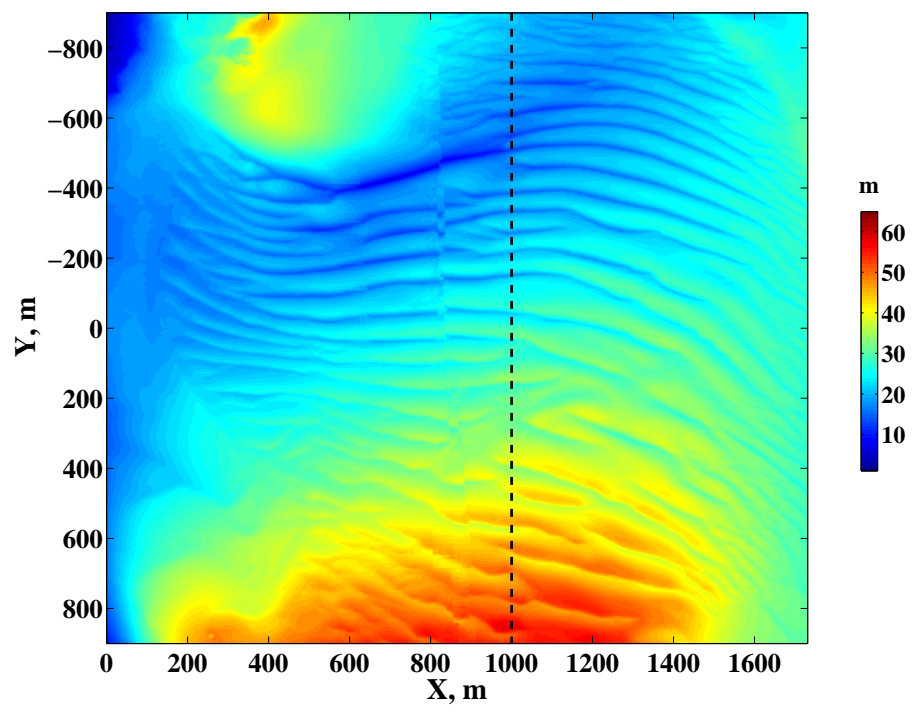

(a)



(b)

Figure 7-10: (a) Bathymetry of the region selected for the acoustic propagation domain (shown by dashed line in Fig. 7-9). (b) Bathymetry transect along $x=1000$ $\mathrm{m}$. 


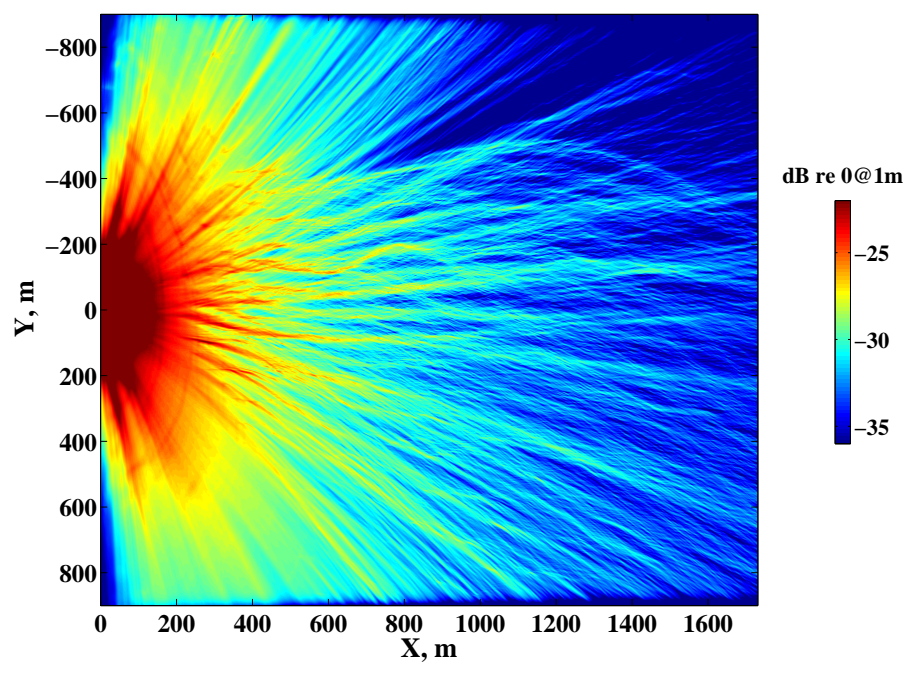

Figure 7-11: Depth integral of the acoustic intensity for a source of frequency $500 \mathrm{~Hz}$ located at $\left(x_{s}, y_{s}, z_{s}\right)=(0,0,10) \mathrm{m}$. 


\section{Chapter 8}

\section{Conclusions}

\subsection{Summary}

The primary focus of this thesis has been to study the physics of three-dimensional low frequency acoustic propagation through shallow water waveguides that contain three common fine scale oceanographic features: ocean internal, surface and bottom waves. The basis of our studies has been the normal mode approach, that allowed us to explain the complexities of the sound wave propagation in terms of simple mechanisms, mainly governed by a two dimensional plane wave Snell's law.

Our primary experimental observations from the SW06 data (Chapter 2) revealed interesting results showing fluctuations with approximately four degrees amplitude in the angle of the signal arrival from a fixed source located $18 \mathrm{~km}$ away from the horizontal receiving array an the azimuth nearly parallel to the dominant direction of the nonlinear internal wave crests. More detailed analysis of similar observations for the same and SW06 other acoustic sources by other researchers [14, 15] agree with our results. The uncertainty about these out of vertical plane acoustic effects was one of the main motivations for our theoretical and numerical studies.

The normal mode approach, whose fundamentals for shallow water waveguides was given in Chapter 3, was employed in Chapter 4 for analytically studying the difference in modal propagation caused by the presence of waves of each type. For this study, we used a simple, but useful idealized model of the shallow water waveguide that allowed 
us to obtain first order corrections to the horizontal wavenumbers of normal modes via perturbation theory. Our results clearly show the difference in the mode number dependence of the horizontal wavenumber effects between wave types. Specifically, we showed that the bottom and surface waves have the biggest influence on the higher order acoustic modes, and their perturbation to the horizontal wavenumbers of the lower order modes weakens evenly with decreasing mode number. On the other hand, one or two mid number modes are most affected by the internal waves, and the perturbation of the higher mode numbers is nearly equal. These results were explained in terms of simple physics.

The horizontal wavenumber perturbation determines the value of the horizontal critical angle - an important parameter of normal modes that plays a key role in "nearly along wave crests" propagation directions. In Chapter 5, we studied coupled normal mode reflection and transmission through straight and parallel waves of two types. Our semi-quantitative studies for a simplified wave shapes and numerical modeling for the realistic canonical wave shapes implicitly showed the importance of the critical angle for the physics of the acoustic interaction with such waves. The physics of the acoustic interaction with straight waves is similar for all wave types. However, the values of wavenumber perturbations (and corresponding critical angles) and its mode number dependence determine the strength of associated effects, and that is different for waves of different types.

Horizontal propagation of normal modes with horizontal grazing angles less than critical leads to an almost total reflection from ocean waves. Depending on the source location relative to a group of parallel waves, this results in either the horizontal Lloyd's mirror (HLM) effect or horizontal acoustic ducting (or antiducting) between the waves. In addition to primary ducting between two parallel waves, we showed that at grazing angles just below the critical values, the effects of horizontal tunneling through the waves and the following reflections from neighbor waves trap the modal energy in secondary ducts. In our numerical simulations, this effect and its explicit dependence on the critical angle value are shown.

On the other hand, at steep grazing angles (approximately 35-90 degrees in our 
examples), strong mode coupling dominates the propagation regime. The sound intensity fluctuations in these directions have been observed in a number of experiments $[3,6,12]$, and are mainly driven by strong mode coupling and its resonant-like behavior. No significant horizontal refraction is observed in this angular region for the ocean wave amplitudes discussed in this work. For intermediate grazing angle range, both horizontal refraction and mode coupling were shown to be noticeable. At these angles, both reflection from the wave fronts and secondary ducting effects are possible through mode coupling mechanisms. However, those were shown to be weak, and acoustic energy propagates mostly without horizontal trapping in this regime.

In addition to straight and parallel waves, in Chapter 6 we studied the physics of the acoustic interaction with crossing internal waves, which are commonly observed on continental shelves. From our analysis, we explained and demonstrated numerically that for wave crossing angles less than double the horizontal critical angle, a significant amount of modal energy, initially ducted by the first group of waves, is either cross-ducted or reflected by the second group of waves. This phenomenon has not been addressed before, and it is of great interest for us to eventually validate it experimentally.

In Chapter 7, we provided results on acoustic propagation through shallow water waveguides that have realistic surface swell waves and real bottom topography with large sand waves. These two fine scale oceanographic features are common for shelves and continental slopes, but have not been considered before in applications to threedimensional acoustics. Surface swells were shown to have a significant impact on the amplitude scintillation of higher order modes in a wide azimuthal range centered at the dominant direction of the wave crests. This scintillation is range dependent due to "smearing" effect of random ducting by local wave crests as well as modal energy coupling into the continuous spectrum at large distances from the source. Our threedimensional modeling of acoustic propagation through the mouth of San Francisco Bay showed that the large sand waves can cause very strong horizontal refraction of low frequency sound waves. Located on a sloping bottom with complicated relief, these waves hold acoustic energy within the ducts, even when they have significant 
curvatures with radii of as low as two kilometers. Our results clearly show the significance of these wave types for underwater acoustics, which we think should be further studied experimentally.

\subsection{Thesis Contributions}

Below I will specify the original contributions, made on my own, but greatly inspired and guided by my advisor.

1. Describing the motion of both the vertical and horizontal line arrays during the SW06 experiment, which was needed for serious beamforming with these arrays,

2. Analyzing the angle of arrival fluctuations for a fixed source in the SW06 experiment, which indicates the existence of out of vertical plane acoustic propagation in shallow water,

3. The analytical analysis of normal mode properties for a simple idealized waveguide and its perturbation due to internal, bottom and surface waves. This provides explicit results that clearly show the first order physical difference between these types of fine scale oceanographic features in applications to low frequency acoustics,

4. A coupled normal mode analytical solution for the acoustic pressure was obtained for a sharp vertical interface dividing an unperturbed idealized waveguide and its perturbation due to one of the wave types. The results qualitatively demonstrate the physics of mode coupled reflection from and transmission through parallel waves,

5. Based on existing algorithms, a fully three-dimensional mode coupling propagation program was developed to evaluate the quantitative importance of mode coupled reflection from, transmission through and ducting between straight parallel waves of different types with an exact solution, 
6. In addition to previously studied horizontal ducting of normal modes, the effect of secondary ducting that is caused mainly by normal mode horizontal tunneling was evaluated numerically for canonical wave shapes, and shown to be the most important mechanism governing the energy leakage from the primary duct,

7. The problem of acoustic propagation through crossing internal waves is simulated numerically for straight internal waves and a variable angle of crossing. It was shown numerically that the reflection from the crossing waves and cross ducting are two important mechanisms that push modal energy out of the primary duct.

8. Three-dimensional numerical modeling of acoustic propagation in presence of long surface swells and bottom sand waves showed the significance of these wave types for low frequency sound propagation applications.

\subsection{Future Directions}

\subsubsection{Analysis and Modeling}

The results we provided in this thesis are for single frequency signals. Although the qualitative physics is similar for a wide range of low frequencies, one should numerically study the broad band three-dimensional acoustic propagation through internal wave crossing structures, surface swells and bottom waves before considering the experimental studies. For instance, path differences between three-dimensional patterns might be resolved by pulse travel times.

For the crossing internal waves, modeling of three-dimensional sound propagation through more realistic waveguides, that incorporate SAR signatures of internal wave curvature and wave crossings, as well as the more detailed vertical structure of the water column inside the wave crossings, will give more exact answers.

Ocean swells (and bottom sediment waves in some parts of the ocean) can also be considered as a spatial distribution of local ducts with random locations, directions and strengths, which are described by a corresponding directional spectrum. Based on 
recent studies by McMahon [73], a diffusion type equation can be applied for studying the acoustic propagation problem statistically in presence of such waves. This is an alternative method of obtaining the statistics of the modal amplitude scintillations due to three-dimensional effects.

\subsubsection{Experimentation}

Three possible experiments aimed at experimentally validating our theoretical and numerical results, and we briefly summarize them.

1. If one wants to capture the effects of secondary ducting or cross-ducting at the internal wave crossings, an optimally deployed "L"-shaped acoustic array and a mobile source that can track the waves in a user defined manner could be an efficient basis for the future experiment. Use of broadband pulses could add some time domain power to resolving the three-dimensional multipaths.

2. For measuring the statistics of modal amplitude scintillations in the presence of long surface swells, a single vertical line hydrophone array and an AUV with mounted acoustic source and tracking equipment will allow one to gather enough data necessary to obtain the azimuthal and range statistics of the modal amplitude scintillations. The three-dimensional scintillations have a very distinct signature versus azimuth and range.

3. A small scale cost-effective experiment with a moored vertical hydrophone array (or even a single hydrophone) and a simple shipping traffic tracking device might be performed in the San Francisco Bay for studying the strong horizontal ducting by the sand waves. The dependence on the space-time variability must be considered, however. 


\section{Appendix A}

\section{Horizontal and Vertical Line Arrays Navigation}

\section{A.1 System Configuration}

In the SW06 experiment, the shape and motion of the vertical (VLA) and horizontal (HLA) line arrays (denoted overall as WHOI array in Fig. 2-1b) were tracked using an acoustic the long baseline (LBL) navigation system [74]. The HLA/VLA receiving system included: an electronics and battery sled (called the Shark because of its shape) connecting to the HLA and VLA; a $79 \mathrm{~m}$ long 16 channel hydrophone VLA, and a $472 \mathrm{~m} 32$ channel hydrophone HLA, anchored by the Shark at one end and by the HLA tail sled at the other end (Fig. 2-2). The closest hydrophone channel to the Shark on the HLA ( $\mathrm{CH} 47)$ is located 3 meters from the sled, followed by 31 evenly spaced (15 meters apart) channels. There is also a $3 \mathrm{~m}$ distance between the last channel on the HLA (CH 47) and the tail sled, followed by a $125 \mathrm{~m}$ long ground cable and $1.5 \mathrm{~m}$ long chain connected to the tail sled. Therefore, there is a $597.5 \mathrm{~m}$ long line connecting the Shark and the tail sled. The Shark was deployed first and then used as anchored weight to keep the HLA tight and straight.

The LBL system consisted of an $11.5 \mathrm{kHz}$ interrogator mounted on the HLA tail

sled and two Benthos TR6000 transponders deployed West $(11.0 \mathrm{kHz})$ and East (12 $\mathrm{kHz}$ ) of the Shark (Fig. A-1). The interrogator and both transponders were deployed 
Table A.1: Deployed and refined positions of the LBL elements.

\begin{tabular}{lll}
\hline & Deployed position & Surveyed or improved position \\
\hline Shark & $391.2516 \mathrm{~N} 732.9824 \mathrm{~W}$ & $391.2627 \mathrm{~N} 732.9887 \mathrm{~W}$ \\
West transponder & $391.3816 \mathrm{~N} 733.2088 \mathrm{~W}$ & $391.3771 \mathrm{~N} 733.2107 \mathrm{~W}$ \\
East transponder & $391.2420 \mathrm{~N} 732.5334 \mathrm{~W}$ & $391.2341 \mathrm{~N} 732.5293 \mathrm{~W}$ \\
Tail sled & $391.5865 \mathrm{~N} 732.9833 \mathrm{~W}$ & $391.5836 \mathrm{~N} 732.9773 \mathrm{~W}$ \\
\hline
\end{tabular}

Table A.2: Nominal positions of the selected hydrophone channels.

\begin{tabular}{ll}
\hline Channel number & Nominal position \\
\hline CH0 & Top of the VLA (Depth 13.5 m) \\
CH6 & $22.5 \mathrm{~m}$ from the VLA top (Depth $36.0 \mathrm{~m}$ ) \\
CH10 & $31.25 \mathrm{~m}$ from the VLA top (Depth $54.75 \mathrm{~m}$ ) \\
CH13 & $63.75 \mathrm{~m}$ from the VLA top (Depth $77.25 \mathrm{~m}$ ) \\
CH17 & HLA, $453 \mathrm{~m}$ from the tail sled (Depth $79 \mathrm{~m}$ ) \\
CH27 & HLA, $303 \mathrm{~m}$ from the tail sled (Depth $79 \mathrm{~m}$ ) \\
CH37 & HLA, $153 \mathrm{~m}$ from the tail sled (Depth $79 \mathrm{~m}$ ) \\
\hline
\end{tabular}

at a depth of one meter above the bottom. After deployment, the positions of the Shark and the West and East transponders were acoustically surveyed from the ship to improve their locations. Table 1 shows the deployed and surveyed positions of the Shark and both transponders, as well as the deployed position of the tail sled. The $11.5 \mathrm{kHz}$ interrogator mounted on the tail sled had no survey because it couldnt be interrogated remotely. The improved position of the tail sled, that is presented in Table A.2, was found from the LBL data in the same manner as the positions of the hydrophone channels below. It follows from the Table A.2 that the distance between the Shark and the tail sled was 594.5 meters, which implies 3 meters of slack in the HLA. Figure A-1 shows a top view of the positions of the two transponders and the interrogator relative to the Shark. Four channels on the VLA (CH0, CH6, CH10, CH13) and three channels on the HLA (CH13, CH27, CH37) were chosen for the LBL reception (circles in Fig. A-1). Table A.2 summarizes nominal locations along the arrays) of these channels.

The sequence of interrogation events is: the interrogator emits an $11.5 \mathrm{kHz} 10 \mathrm{~ms}$ ping that is then retransmitted by the East and West transponders at frequencies of 




Figure A-1: Top view of the LBL system elements and selected hydrophone channels for LBL navigation.

11.0 and $12.0 \mathrm{kHz}$ respectively. Each designated channel on the HLA(VLA) receives the signals and stores corresponding travel time. The turnaround time of the TR6000 Benthos transponder is specified to be $2.5 \mathrm{~ms}$. There is also $10 \mathrm{~ms}$ needed to detect the pulse, so that the total travel time through each of the two transponders is the sum of the travel time from interrogator to transponder, travel time from the transponder to the channel on the HLA(VLA), and a total turnaround time of $12.5 \mathrm{~ms}$. Travel times from the interrogator to the selected channels do not include any of these additional delays.

\section{A.2 VLA Navigation}

Four hydrophone channels on the VLA (CH0, CH6, CH10 and CH13) were selected to receive the LBL signals. Channel 13 was nominally located only one meter above the bottom and was therefore considered to be fixed. Travel time to this hydrophone was used to get an improved position of the tail sled that is shown in Table A.2. This position was calculated in the same manner as the positions of the HLA channels 
in the next section. Travel times to the VLA channels CH6 and CH10 had much larger variance than the travel times to the top VLA channel CH0 for all three paths. Assuming the strain in the VLA due a 37.5" diameter floating steel sphere is enough to keep the VLA cable straight, we decided not to consider those travel times further and only used the travel times to the top VLA channel CH0 for VLA navigation.

The one leg corrected travel times can be written as

$$
\begin{aligned}
T_{W} & =t_{W}-0.0125-t_{I-W}, \\
T_{E} & =t_{E}-0.0125-t_{I-E}, \\
T_{I} & =t_{I},
\end{aligned}
$$

where $t_{W}, t_{E}$ and $t_{I}$ are the total travel times from the signal interrogation until its reception at channel $\mathrm{CH} 0$ for the two-leg paths through the West transponder, East transponder and the direct path from the interrogator respectively, and $t_{I-W}$ and $t_{I-E}$ are the travel times between the interrogator and West and East transponders respectively. As an illustration, Fig. A-2 shows total travel time series $t_{I}$ and $t_{E}$ to the channel $\mathrm{CH}$. One can see from this figure that the variance of the total travel time through the transponder is greater than for the direct path. In order to understand the arrival time structure, as well as to aid in calculating the interrogator to transponder travel times $t_{I-W}$ and $t_{I-E}$, a ray tracing method [1] was used. In our calculations, we used the water column sound speed at the Shark position provided by the WHOI data (available online at the SW06 website). Assuming a locally range independent water column, eigenrays from the interrogator to each of the transponders were found, using the Bellhop [75] ray tracing code. Figure A-3 shows typical eigenrays connecting the interrogator with the West (center plot) and East (right plot) transponders for one of the selected water column sound speed (left plot). Between the interrogator and the West transponder, there was usually one along bottom water borne eigenray (blue line) and one surface reflected eigenray(black line) found. However, our modeling often showed one additional water borne refracted eigenray (blue refracted ray in the right plot) connecting the interrogator with the East transponder. At the West twoleg path, along bottom eigenray was dominant over the entire time of experiment. 


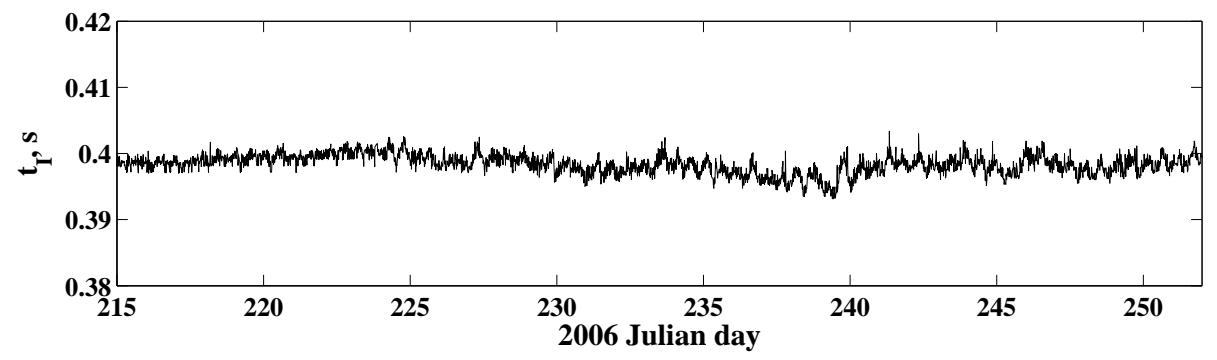

(a)

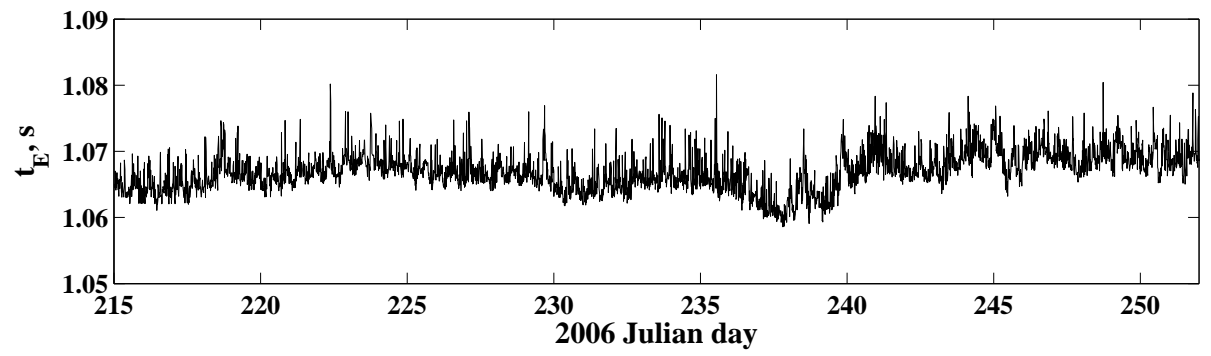

(b)

Figure A-2: Example of the total travel time from the interrogator to the top VLA hydrophone channel $\mathrm{CHO}$ for the direct path (upper panel) and for the two-leg path through the East transponder (lower panel).

On the other hand, both water borne eigenrays were often considered on the East two-leg path, since the travel time difference along these eigenrays was comparable with the variation of the total travel time along this path. As a result, up to two possible values are computed for $T_{I-E}$ and one for $T_{I-W}$ are available for our further calculations.

After one-leg travel times $T_{W}, T_{E}$ and $T_{I}$ are found, ray tracing was performed again, but in a "ray fan" mode. In Fig. A-4, a ray fan (right plot) is plotted for one of the sound speed columns (same water column example as in Fig. A-3). The circles on the rays denote possible locations of the channel $\mathrm{CH} 0$ in the vertical planes connecting the East transponder (left group), the interrogator (central group) and the West transponder (right group) with the Shark according to the integral of the travel time along the rays. The plots in the Fig. A-5 show these patterns separately for each of the three paths. The red rhomb denotes the nominal position of the top channel CHO (assuming the upright position of the VLA) on each of the vertical planes. Blue 


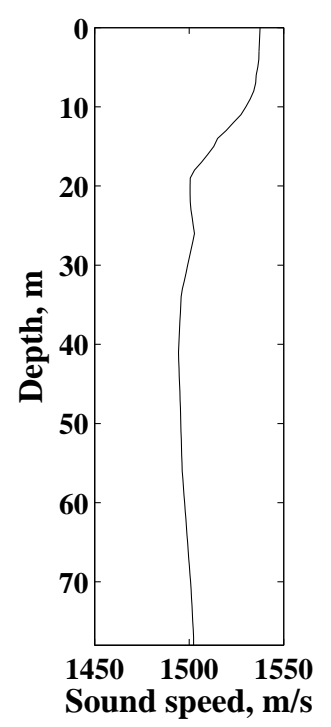

(a)

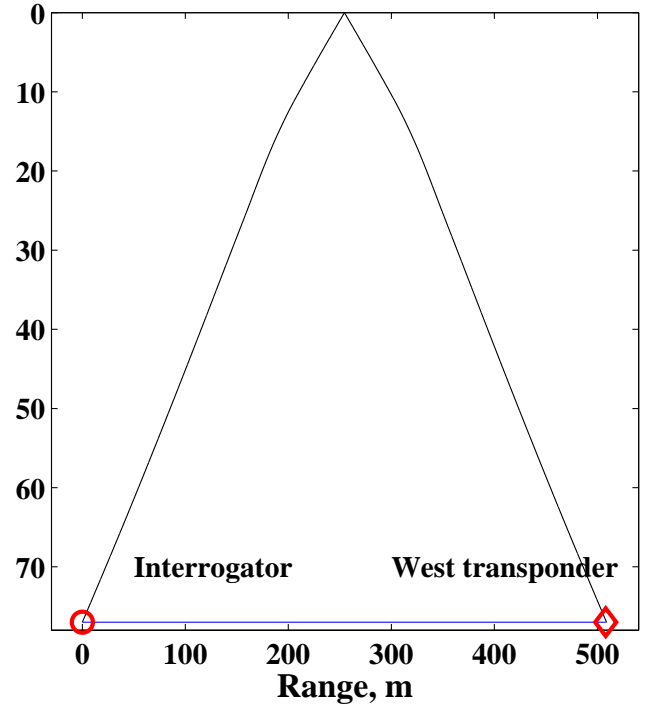

(b)

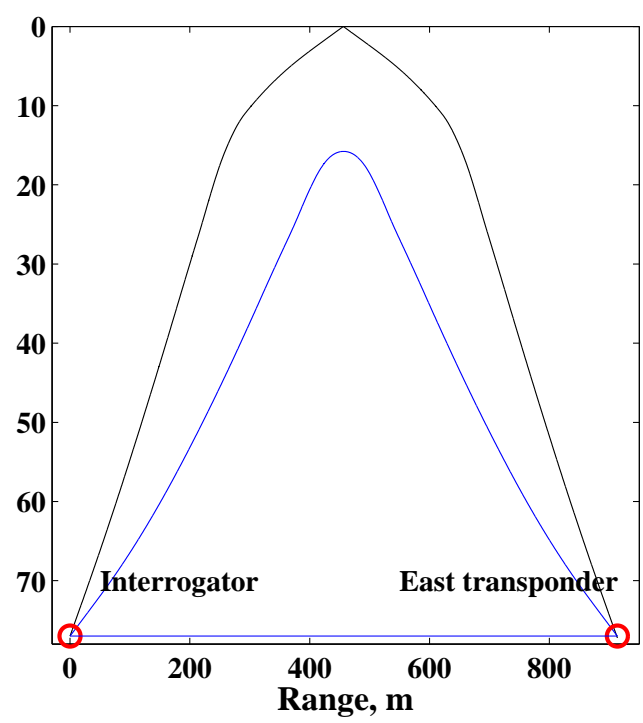

(c)

Figure A-3: One of the selected sound speed columns (a) and the corresponding eigenrays connecting the interrogator with the West transponder (b) and the interrogator with the East transponder (c). 


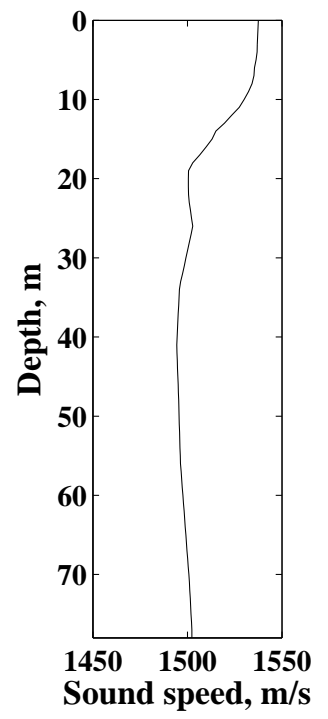

(a)

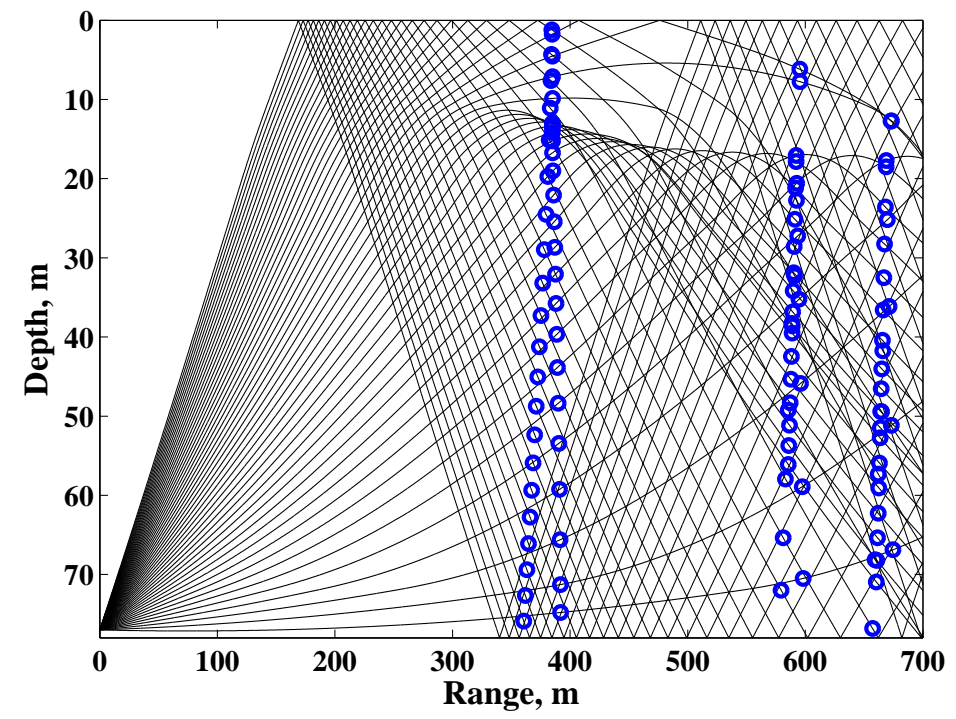

(b)

Figure A-4: One of the sound speed columns at the VLA (a) and computed acoustic "ray fan" (b). Left, central and right groups of circles correspond to the possible locations of the channel $\mathrm{CH} 0$ according to the travel time integral along each ray for the paths East transponder-to-CH0, interrogator-to-CHO and West transponder-toCHO respectively.

and black circles represent water borne and surface reflected rays respectively. As one can see from the plots, the arrival structure is complicated and different for each path. In this particular example, water borne rays do not reach the nominal depth of the channel $\mathrm{CHO}$ for the East transponder-to- $\mathrm{CH} 0$ and interrogator-to- $\mathrm{CH} 0$ paths (shadow zone). On the other hand, both surface reflected and water borne rays are available for the West transponder-to-CH0 path. The structure of these patterns was changing with time as the water column sound speed was changing. We also note that we had up to two possible corrected travel times $T_{I-E}$ which doubles the number of possible horizontal ranges from the East transponder to the channel $\mathrm{CH}$. In order to find the correct horizontal range from the West transponder, the interrogator, or the East transponder to the channel $\mathrm{CH}$, we selected only the rays that reach the $\pm 1 \mathrm{~m}$ range of the nominal depth of the channel $\mathrm{CH} 0$. If more than one such ray was available (as in the example of the West transponder-to-CH0 paths shown in the top panel of Fig. A-5) or if more than one corrected travel time is available (two possible values of $T_{I-E}$ ), we manually selected the most probable horizontal range 


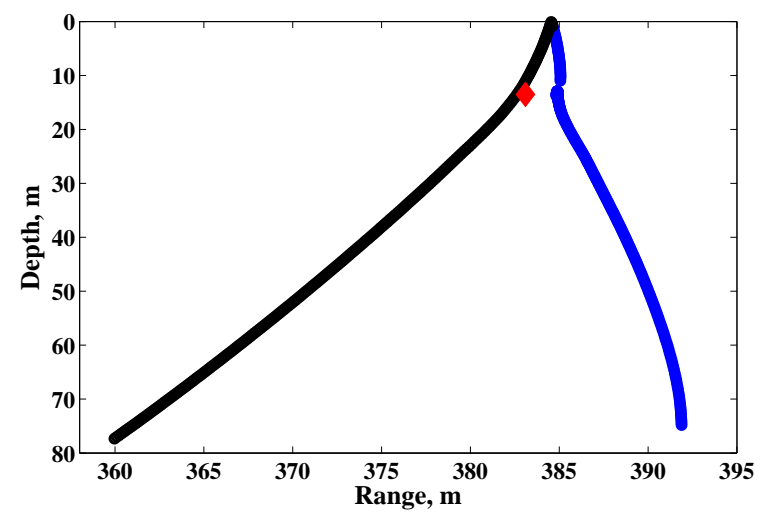

(a)

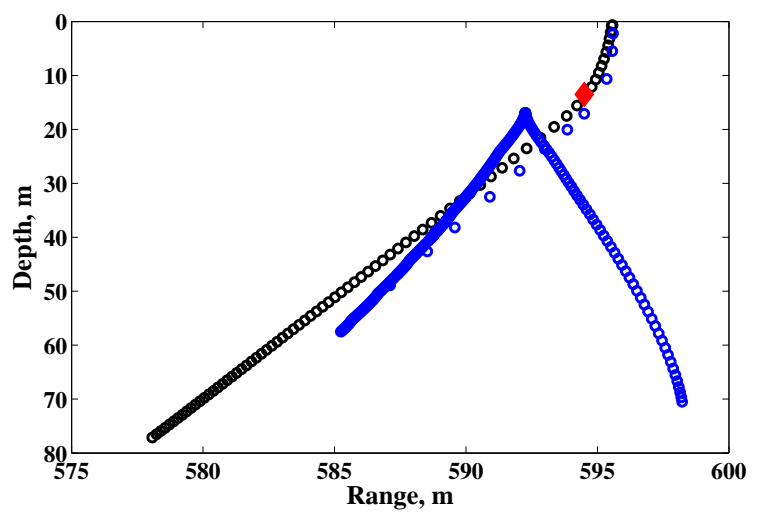

(b)

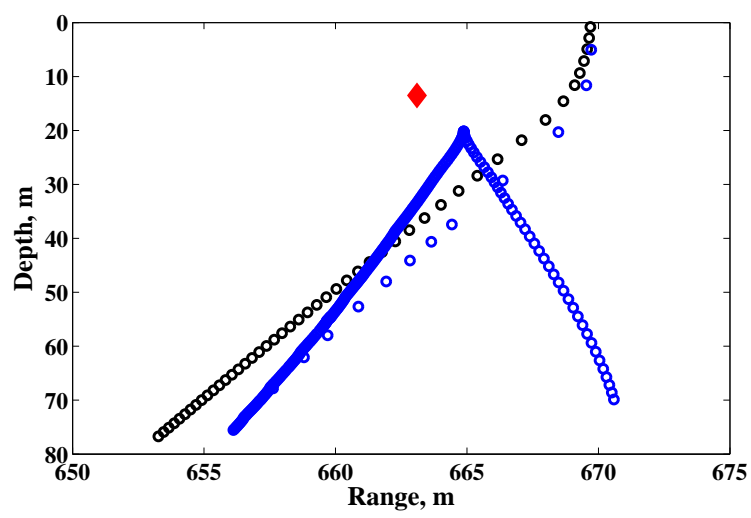

(c)

Figure A-5: Possible locus of the channel $\mathrm{CH} 0$ in the vertical planes connecting the West transponder and $\mathrm{CHO}$ (a), the Interrogator and $\mathrm{CHO}$ (b) and the East transponder and $\mathrm{CHO}$ (c). Blue and black colors denote water borne and surface reflected rays respectively. Red rhombs indicates the nominal position of the channel $\mathrm{CH}$. 


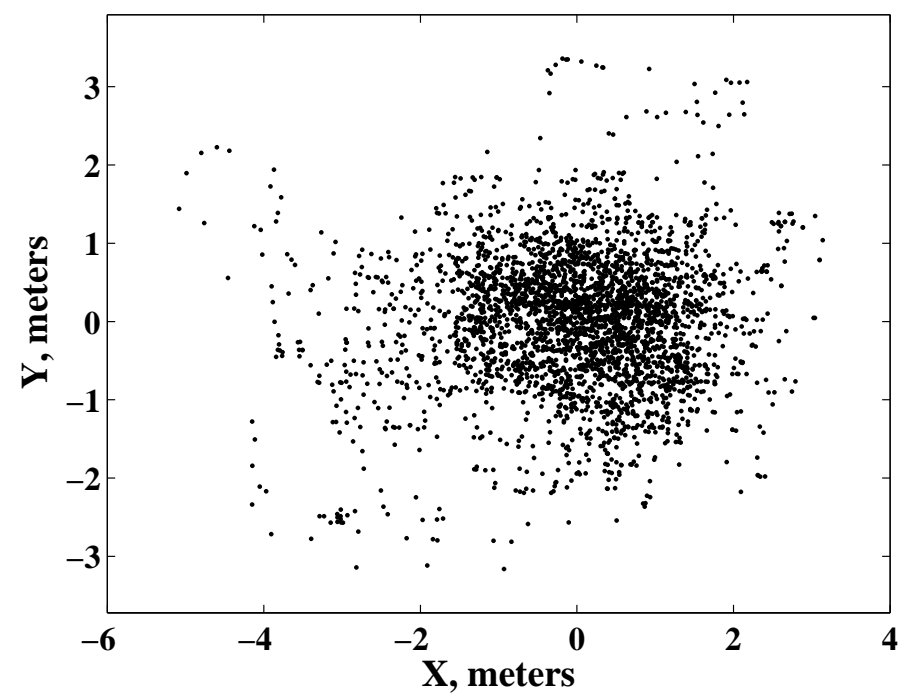

Figure A-6: Horizontal positions of the top VLA hydrophone channel CH0. The $y$-axis points true North and the $x$-axis points true East.

from the interrogator (or West or East transponder) to the channel $\mathrm{CH} 0$ according to the geometrical constraint of maximum array tilt (we used $15 \mathrm{deg}$ as a maximum tilt constraint that would correspond to the strong currents of $2 \mathrm{~m} / \mathrm{s}$, given the flotation sphere radius and weight). A median filter with a window length of three hours was then applied to the selected ranges. The final horizontal position of $\mathrm{CH} 0$ was found by a least square fit triangulation, see Fig. A-6. The standard deviation of the horizontal displacement of $\mathrm{CH} 0$ is $0.8 \mathrm{~m}$ and the maximum horizontal excursion is $5.3 \mathrm{~m}$. This results in a standard deviation of the VLA tilt of 0.6 deg and maximum tilt of 3.9 deg.

\section{A.3 HLA Navigation}

For tracking the selected HLA channels CH17, CH27 and CH37, we used the corrections $T_{I_{W}}$ and $T_{I-E}$ found during the localization of the channel $\mathrm{CH} 0$ in the previous section. Ray tracing was performed for the corrected one leg travel times for these channels, in the same manner as we did for the channel CH0. Arrival patterns, qualitatively similar to Fig. A-5, were obtained for these channels, but in this case the nominal depth is $77 \mathrm{~m}$ since the HLA lay on the seabed. As we can see from Fig. A-5, 
at larger depths the range bias noted between selecting different multipaths is larger for deeper hydrophones, and it is biggest at the seabed. This is consistent with much larger variance of the one leg corrected travel times to some of the HLA channels (as an example, the East transponder-to-CH27) than to the VLA channel CH0. In order to pick the proper eigenray from each of the transponders and the interrogator to the selected HLA channel, the following method was used. A moving average with a window length of four hours was calculated for each of the eigenrays' horizontal range and for each of three paths. By looking at these, the dominating eigenray was chosen at each of the three paths by means of a best least squares fit. For some paths, the along bottom water borne eigenray reception dominated, whereas for the other paths it was the refracted water borne ray. Although there was a dominant eigenray for each particular path, sometimes others were picked up by the receiver. As an example, Fig. A-7 shows the horizontal range from the East transponder to $\mathrm{CH} 27$ path corresponding to the water borne along bottom eigenray (green), a refracted water borne eigenray (blue) and a surface reflected eigenray (black). By analyzing the moving average lowpass filtered horizontal ranges for each of the eigenrays, the water borne refracted eigenray was shown to be dominant in this example. The yellow line represents the low lowpass filtered ranges of the dominant ray. One can see from the figure that sometimes the surface reflected or along bottom water borne eigenrays have horizontal ranges closer to the lowpass filtered ranges of the dominant ray. In such cases, we selected these rays instead of the dominant ray for our computations. A final combination of the horizontal ranges for selected eigenrays is shown by the red dots in Fig. A-7. A similar calculation was performed for other HLA channels.

Applying least square triangulation to the selected horizontal ranges, locations of channels CH17, CH27 and CH37 are found and plotted in Fig. A-8 in Cartesian coordinates, where the Shark is taken as the origin, the $y$-axis points True North and the $x$-axis points True East. As we can see from the plots, the spread in the $y$-components of the locations found is less in the $x$-components, which is consistent with only three meters of slack in the total length of the HLA, connecting cables and chains. Referring to the x-direction distribution, we note two distinct groups at 
Table A.3: Average values and standard deviations for the coordinates of the HLA hydrophone channels CH17, CH27 and CH37.

\begin{tabular}{|l|l|c|c|c|c|}
\hline & Period & $\langle X\rangle, \mathrm{m}$ & $\mathrm{STD}(X), \mathrm{m}$ & $<Y>, \mathrm{m}$ & $\mathrm{STD}(Y), \mathrm{m}$ \\
\hline CH17 & Deployment - Recovery & 0 & 1.2 & 451.1 & 0.6 \\
\hline \multirow{3}{*}{ CH27 } & Deployment - Aug 19 21:39 UTC & -7.9 & 1.9 & 302.3 & 0.3 \\
& Aug 19 21:39 - Sep 2 10:36 UTC & -1.2 & 1.1 & 302.0 & 0.4 \\
& Sep 2 10:36 UTC - Recovery & -5.6 & 1.1 & 302.2 & 0.4 \\
\hline \multirow{3}{*}{ CH37 } & Deployment - Aug 19 21:39 UTC & -7.6 & 2.3 & 151.9 & 0.1 \\
& Aug 19 21:39 - Sep 2 10:36 UTC & 4.4 & 1.3 & 151.5 & 0.1 \\
& Sep 2 10:36 UTC - Recovery & -6.4 & 1.1 & 151.9 & 0.1 \\
\hline
\end{tabular}

both the CH27 and CH37 locations. This indicates the movement of the HLA along the $x$-axis, during which the Southern part of the HLA moved over greater distance than its Northern part. From Fig. A-9, one can see that there were actually two shifts of the HLA: one towards the East on 2006 Julian day 231 (August 19, 2006), and another towards the West on 2006 Julian day 245 (September 2, 2006). Both shifts indicate a larger movement of channel CH37 and very small or no movement of channel CH17.

To support the results obtained, Fig. A-10 shows a very good correlation between the two shifts seen in Fig. A-9 and the Eastern component of the ocean current, recorded with an Acoustic Doppler Current Profiler at the $68 \mathrm{~m}$ depth bin at the nearby mooring SW30 location (see [13] for the mooring configuration details). We conclude from this data that the two shifts of the HLA were induced by bottom currents on August 19 and September 2.

Although we partially corrected the multipath uncertainties in the horizontal ranges, we were not able to describe any other positive movements of the HLA. Therefore, we suggest that for practical purposes, the average positions of the channels CH27 and CH37 are used for each of the three time periods: 1) deployment until the first shift on August 19 at 21:30, 2) the first shift until the second shift on September 2 at 11:00, and 3) from the second shift until recovery. Channel CH17 did not show any noticeable movements during the entire time of experiment. Table A.3 summarizes the average coordinates of the channels CH17, CH27 and CH37 and its standard deviations for each of the three selected periods. 


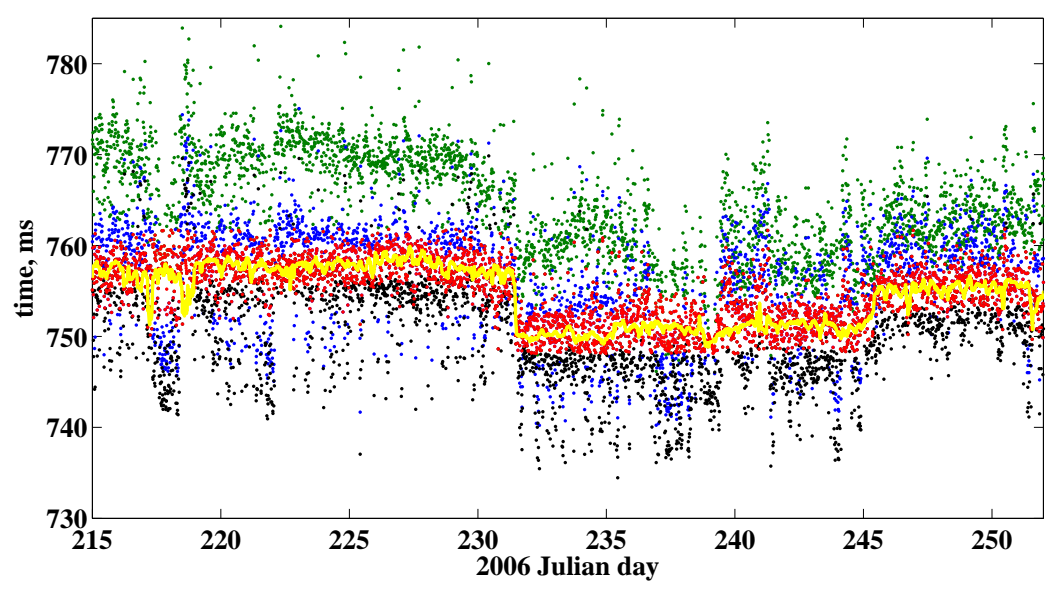

Figure A-7: Example of the horizontal ranges for the eigenrays connecting the East transponder and channel CH27. Green and blue dots represent along bottom and refracted water borne eigenrays, and black dots represent surface reflected eigenrays. The eellow line is the low pass filtered horizontal ranges for the dominant (blue) eigenray. Red dots show a combination of selected horizontal ranges used for the final localization.
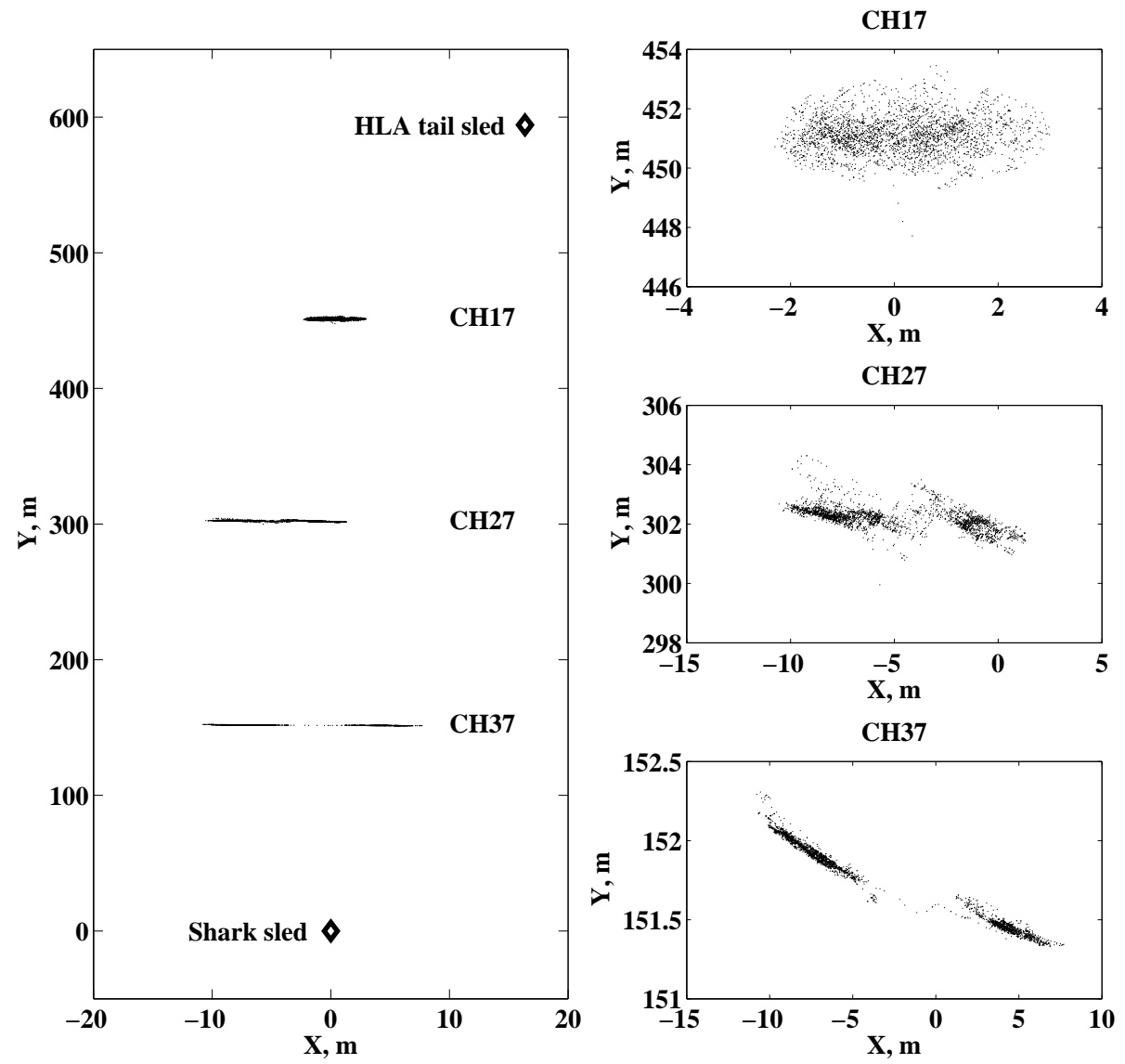

Figure A-8: Localized positions of HLA channels CH17, CH27 and CH37. 

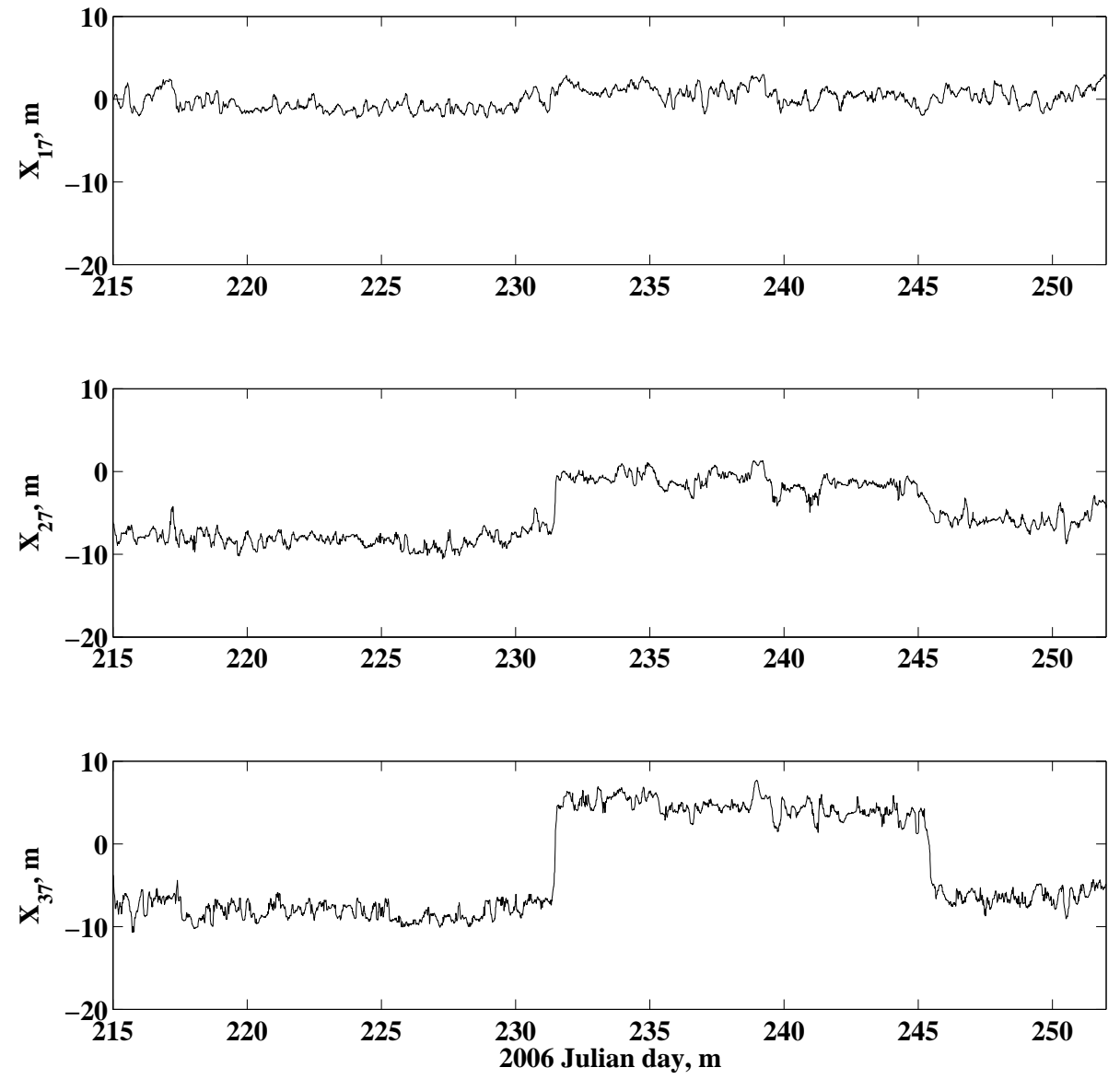

Figure A-9: X-component of HLA channels locations (CH17, CH27 and CH37).

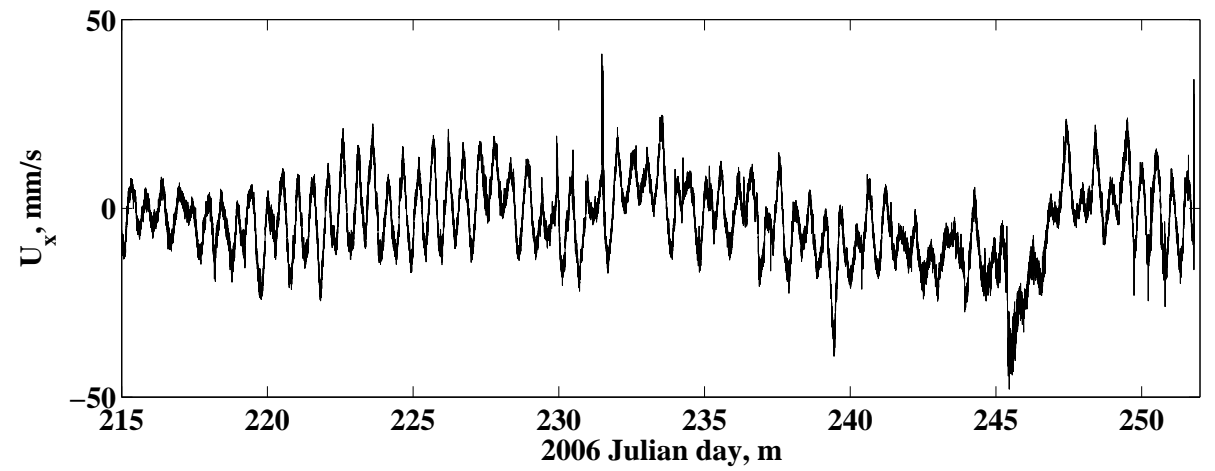

Figure A-10: Eastern component of the ocean current for the $68 \mathrm{~m}$ depth bin at the SW30 mooring. 


\section{Appendix B}

\section{Three-Dimensional Mode Coupling in Environments Changing in Two Dimensions}

Here we describe a useful algorithm for computing three-dimensional coupled mode solutions for the acoustic pressure in waveguides with laterally varying properties along the $y$-axis and independent of the $x$-axis. Assume waveguide changes to be within the segment $\left[\begin{array}{ll}y_{a} & y_{b}\end{array}\right]$ such that

$$
\begin{gathered}
c(x, y, z)= \begin{cases}c^{a}(z) & , y \leq y_{a}, \\
c(y, z) & , y_{a}<y<y_{b}, \\
c^{b}(z) & , y \geq y_{b}\end{cases} \\
\rho(x, y, z)= \begin{cases}\rho^{a}(z) & , y \leq y_{a} \\
\rho(y, z) & , y_{a}<y<y_{b}, \\
\rho^{b}(z) & , y \geq y_{b} .\end{cases}
\end{gathered}
$$

Similar to Section 5.3.2, the one-dimensional I.F.T. operator (Eq. 5.12) can be applied to both sides of Eq. (3.33), and we can rewrite the resultant two-dimensional 
separated Helmholtz equation [53]:

$$
\rho \frac{\partial}{\partial y}\left[\frac{1}{\rho} \frac{\partial p}{\partial y}\right]+\rho \frac{\partial}{\partial z}\left[\frac{1}{\rho} \frac{\partial p}{\partial z}\right]+\left[\frac{\omega^{2}}{c^{2}}-k_{x}^{2}\right] p\left(k_{x}, y, z\right)=-\frac{\delta\left(y-y_{s}\right) \delta\left(z-z_{s}\right)}{2 \pi}
$$

Our initial three-dimensional mode coupling problem is now divided into many twodimensional problems, one for each $k_{x}$, that can be solved using known techniques. One of them is the spectral parabolic equation $(\mathrm{PE})$, introduced by Orris and Collins [76] for studying three-dimensional acoustic propagation over a sloping bottom. Although the spectral PE method is robust, it has certain angular limitations, and we want to provide the most accurate solution to our three-dimensional problem. To do this, we employ the two-way coupled mode method of solving each of the $x$-reduced problems [54]. Following the logic of the two-dimensional two-way mode coupling algorithm [55,33], we divide our waveguide into $N+1$ segments by $N$ vertical plane interfaces $y=y_{j}, j=1 . . N$ such that $y_{1}=y_{a}, y_{N}=y_{b}$ (Fig. B-1). It is further assumed that the waveguide properties don't change significantly within each segment $j$ and are locally range independent with density and sound speed columns $\rho^{j}(z)$ and $c^{j}(z)$ respectively, horizontal modal wavenumbers $k_{r m}^{j}$, and a mode function set $\Psi_{m}^{j}(z)$. By placing an acoustic source into segment $j_{s}$ and neglecting the contribution from the continuous part of the spectrum, the acoustic pressure field in segment $j$ is

$$
\tilde{p}^{(j)}\left(k_{x}, y, z\right)=\sum_{m=1}^{M}\left[\Phi_{m}^{(j)+} e^{i k_{y m}^{j}\left(k_{x}\right)\left(y-y_{j}\right)}+\Phi_{m}^{(j)-} e^{i k_{y m}^{j}\left(k_{x}\right)\left(y_{j}-y\right)}\right] \Psi_{m}^{j}(z)+\delta(j-s) \tilde{p}^{s}
$$

where the source contribution is

$$
\tilde{p}^{s}\left(k_{x}, y, z\right)=\frac{i}{4 \pi \rho\left(z_{s}\right)} \sum_{m=1}^{M} \Psi_{m}^{j_{s}}\left(z_{s}\right) \Psi_{m}^{j_{s}}(z) \frac{e^{i k_{y m}^{j_{s}}\left(k_{x}\right)\left|y-y_{s}\right|}}{k_{y m}^{j_{s}}\left(k_{x}\right)}
$$

In the above, $\mathrm{M}$ is the number of propagating modes, and the $y$-component of the modal wavenumber in segment $j$ is defined (similarly to Section 5.2) as

$$
k_{y m}^{j} \equiv \begin{cases}\sqrt{\left(k_{r m}^{j}\right)^{2}-k_{x}^{2}}, & \left|k_{x}\right| \leq k_{r m}^{j} \\ i \sqrt{k_{x}^{2}-\left(k_{r m}^{j}\right)^{2}}, & \left|k_{x}\right| \geq k_{r m}^{j}\end{cases}
$$




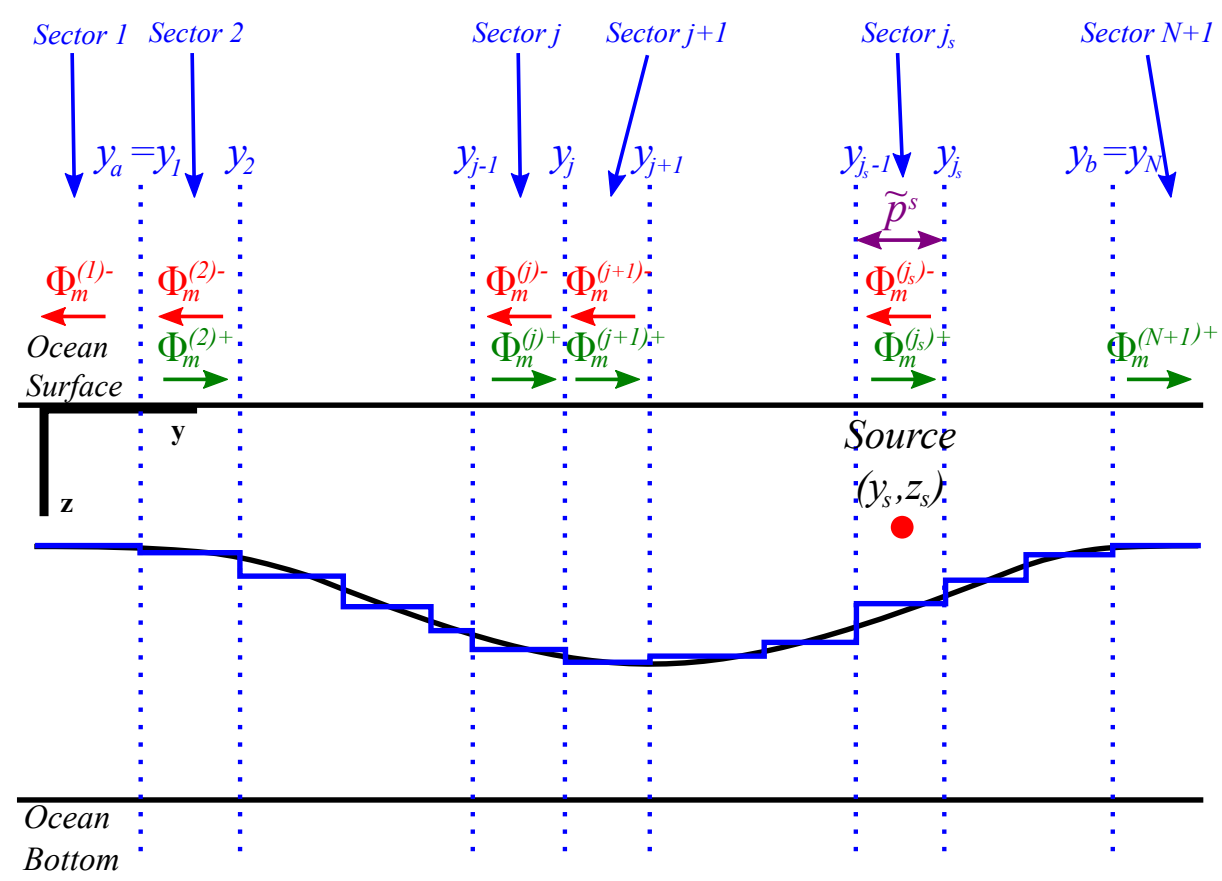

Figure B-1: The range dependent part of the waveguide $\left[\begin{array}{ll}y_{a} & y_{b}\end{array}\right]$ is divided by $N$ vertical interfaces $y_{j}, j=1 . . N$ into $N+1$ segments such that $y_{1}=y_{a}, y_{N}=y_{b}$. Within each segment, the waveguide properties don't change significantly and are thus locally range independent. The solution within each homogeneous segment $j$ is a sum of right and left going plane waves with amplitudes $\Phi_{m}^{(j)+}$ and $\Phi_{m}^{(j)-}$ respectively. Segment $j_{s}$ containing the acoustic source has an additional source term $\tilde{p}^{s}$ in the solution. 
Using the matrix notation formalism in the two-dimensional version of this algorithm [33], appropriate boundary conditions at interface $j$ for acoustic pressure and $y$-component of particle velocity can be written as

$$
\boldsymbol{\Phi}^{(j+1)+}+\mathbf{E}_{2}^{j+1} \boldsymbol{\Phi}^{(j+1)-}+\mathbf{S}_{1} \delta_{j_{s}, j+1}=\tilde{\mathbf{C}}^{j}\left(k_{x}\right)\left(\mathbf{E}_{1}^{j} \boldsymbol{\Phi}^{(j)+}+\boldsymbol{\Phi}^{(j)-}+\mathbf{S}_{2} \delta_{j_{s}, j}\right),
$$

and

$$
\boldsymbol{\Phi}^{(j+1)+}-\mathbf{E}_{2}^{j+1} \boldsymbol{\Phi}^{(j+1)-}-i \mathbf{S}_{1} \delta_{j_{s}, j+1}=\hat{\mathbf{C}}^{j}\left(k_{x}\right)\left(\mathbf{E}_{1}^{j} \boldsymbol{\Phi}^{(j)+}-\boldsymbol{\Phi}^{(j)-}+i \mathbf{S}_{2} \delta_{j_{s}, j}\right),
$$

respectively, where $\delta_{j_{s}, j}$ denotes the Kronecker symbol as before. Vectors $\boldsymbol{\Phi}^{(j)+}$ and $\boldsymbol{\Phi}^{(j)-}$ consist of coefficients $\Phi_{m}^{(j)+}$ and $\Phi_{m}^{(j)-}$ respectively, the propagator matrices $\mathbf{E}_{1}^{j}$ and $\mathbf{E}_{2}^{j+1}$ are

$$
\begin{gathered}
\mathbf{E}_{1}^{j}=\operatorname{diag}\left(e^{i k_{y m}^{j}\left(k_{x}\right)\left(y_{j}-y_{j-1}\right)}\right), \\
\mathbf{E}_{2}^{j+1}=\operatorname{diag}\left(e^{i k_{y m}^{j+1}\left(k_{x}\right)\left(y_{j+1}-y_{j}\right)}\right),
\end{gathered}
$$

and the coupling matrices $\tilde{\mathbf{C}}^{(j)}$ and $\hat{\mathbf{C}}^{l}$ are

$$
\begin{gathered}
\tilde{\mathbf{C}}_{l m}^{j}\left(k_{x}\right)=\int \frac{\Psi_{l}^{j+1}(z) \Psi_{m}^{j}(z)}{\rho^{j+1}(z)} d z \\
\hat{\mathbf{C}}_{l m}^{j}\left(k_{x}\right)=\frac{k_{y m}^{j}\left(k_{x}\right)}{k_{y l}^{j+1}\left(k_{x}\right)} \int \frac{\Psi_{l}^{j+1}(z) \Psi_{m}^{j}(z)}{\rho^{j}(z)} d z .
\end{gathered}
$$

Vectors $\mathbf{S}_{1}$ and $\mathbf{S}_{2}$ are the source contributions at the interfaces $j_{s}-1$ and $j_{s}$ and consist of elements $s_{1, m}, m=1 . . M$ and $s_{2, m}, m=1 . . M$ respectively, where

$$
\begin{aligned}
s_{1, m} & =\frac{i \Psi_{m}^{j_{s}}\left(z_{s}\right) e^{i k_{y m}^{j_{s}}\left(y_{s}-y_{j_{s}-1}\right)}}{4 \pi \rho\left(z_{s}\right) k_{y m}^{j_{s}}\left(k_{x}\right)} \\
s_{2, m} & =\frac{i \Psi_{m}^{j_{s}}\left(z_{s}\right) e^{i k_{y m}^{j_{s}}\left(y_{j_{s}}-y_{s}\right)}}{4 \pi \rho\left(z_{s}\right) k_{y m}^{j_{s}}\left(k_{x}\right)}
\end{aligned}
$$


The Sommerfeld radiation condition implies that

$$
\begin{aligned}
\boldsymbol{\Phi}^{(1)-} & =\mathbf{0}, \\
\boldsymbol{\Phi}^{(N+1)+} & =\mathbf{0} .
\end{aligned}
$$

By combining all the equations together into one matrix block form, one gets

$$
\left[\begin{array}{cccccccccc}
\mathbf{R}_{2}^{1} & -\mathbf{I} & \mathbf{0} & & & & & & & \\
\mathbf{R}_{4}^{1} & \mathbf{0} & -\mathbf{I} & & & & & & & \\
\ddots & \ddots & \ddots & \ddots & & & & & & \\
& & \ddots & \ddots & \ddots & \ddots & & & & \\
& & & \mathbf{R}_{1}^{j_{s}-1} & \mathbf{R}_{2}^{j_{s}-1} & -\mathbf{I} & \mathbf{0} & & & \\
& & & \mathbf{R}_{3}^{j_{s}-1} & \mathbf{R}_{4}^{j_{s}-1} & \mathbf{0} & -\mathbf{I} & & & \\
& & & & & \mathbf{R}_{1}^{j_{s}} & \mathbf{R}_{2}^{j_{s}} & -\mathbf{I} & \mathbf{0} & \\
& & & & & \mathbf{R}_{3}^{j_{s}} & \mathbf{R}_{4}^{j_{s}} & \mathbf{0} & -\mathbf{I} & \\
& & & & & \ddots & \ddots & \ddots & \ddots & \\
& & & & & & \ddots & \ddots & \ddots & \ddots \\
& & & & & & & \mathbf{R}_{1}^{N} & \mathbf{R}_{2}^{N} & -\mathbf{I} \\
& & & & & & & \mathbf{R}_{3}^{N} & \mathbf{R}_{4}^{N} & \mathbf{0}
\end{array}\right]\left[\begin{array}{l}
\boldsymbol{\Phi}^{(1)-} \\
\boldsymbol{\Phi}^{(2)+} \\
\boldsymbol{\Phi}^{(2)-} \\
\vdots \\
\boldsymbol{\Phi}^{\left(j_{s}-1\right)+} \\
\boldsymbol{\Phi}^{\left(j_{s}-1\right)-} \\
\boldsymbol{\Phi}^{\left(j_{s}\right)+} \\
\boldsymbol{\Phi}^{\left(j_{s}\right)-} \\
\vdots \\
\boldsymbol{\Phi}^{(N-1)+} \\
\boldsymbol{\Phi}^{(N-1)-} \\
\boldsymbol{\Phi}^{(N)+}
\end{array}\right]=\left[\begin{array}{c}
\mathbf{0} \\
\vdots \\
\\
\mathbf{0} \\
\mathbf{R}_{5} \mathbf{S}_{1} \\
\mathbf{R}_{6} \mathbf{S}_{1} \\
\mathbf{R}_{7} \mathbf{S}_{2} \\
\mathbf{R}_{8} \mathbf{S}_{2} \\
\mathbf{0} \\
\\
\vdots \\
\mathbf{0}
\end{array}\right],
$$

where

$$
\begin{aligned}
\mathbf{R}_{1}^{j} & =\frac{\tilde{\mathbf{C}}^{j}+\hat{\mathbf{C}}^{j}}{2} \mathbf{E}_{1}^{j}, \\
\mathbf{R}_{2}^{j} & =\frac{\tilde{\mathbf{C}}^{j}-\hat{\mathbf{C}}^{j}}{2}, \\
\mathbf{R}_{3}^{j} & =\left(\mathbf{E}_{2}^{j+1}\right)^{-1} \frac{\tilde{\mathbf{C}}^{j}-\hat{\mathbf{C}}^{j}}{2} \mathbf{E}_{1}^{j}, \\
\mathbf{R}_{4}^{j} & =\left(\mathbf{E}_{2}^{j+1}\right)^{-1} \frac{\tilde{\mathbf{C}}^{j}+\hat{\mathbf{C}}^{j}}{2} \\
\mathbf{R}_{5} & =\mathbf{0} \\
\mathbf{R}_{6} & =-\left(\mathbf{E}_{2}^{j_{s}}\right)^{-1} \\
\mathbf{R}_{7} & =\frac{\tilde{\mathbf{C}}^{j_{s}}+\hat{\mathbf{C}}^{j_{s}}}{2}, \\
\mathbf{R}_{7} & =\left(\mathbf{E}_{2}^{j_{s}+1}\right)^{-1} \frac{\tilde{\mathbf{C}}^{j_{s}}-\hat{\mathbf{C}}^{j_{s}}}{2}
\end{aligned}
$$




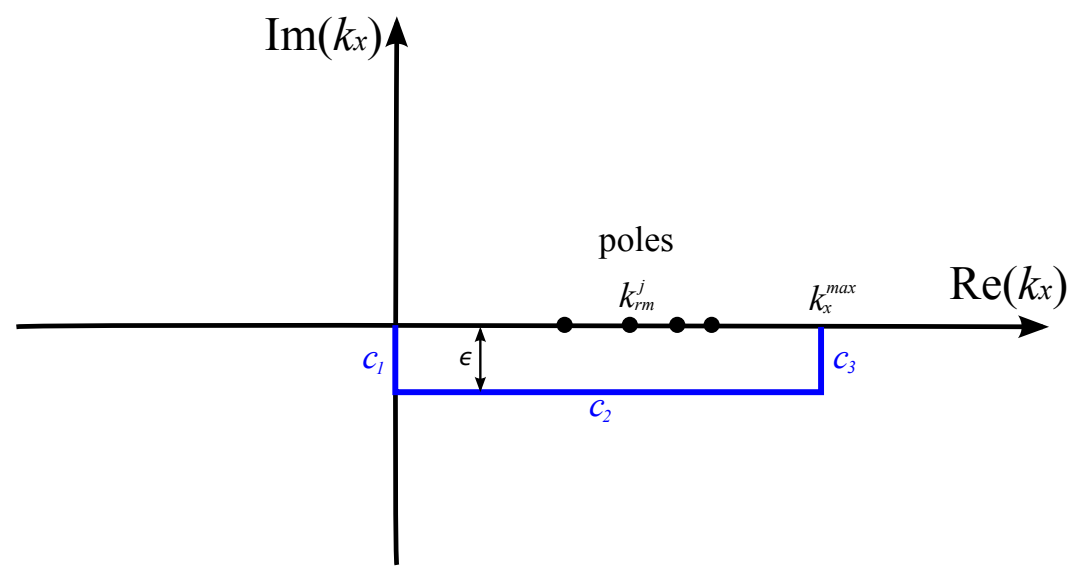

Figure B-2: New integration contour shifted into the complex plane away from the poles.

and $\mathbf{I}$ is the identity matrix. Having solved the block matrix equation above for multiple evenly spaced values of $k_{x}$, the pressure kernel is constructed using B.4 and converted to $p(x, y, z)$ with the one-dimensional F.T. operator (Eq. (5.18)).

In the absence of physical attenuation in the system (bottom loss is a good example), matrix Eq. (B.15) has a singular solution in segment $j$ when

$$
k_{x}=k_{r m}^{j}, m=1 . . M
$$

Although including physical attenuation smooths the kernel slightly, it still requires a large number of sampling points to avoid aliasing. Another method that requires much fewer sampling points consists of shifting the integration contour into the complex plane $[77,33]$ by the offset $\epsilon$ (Fig. B-2). The new integration contour consists of three sections $c_{1}, c_{2}$, and $c_{3}$. With a proper choice of the upper integration limit, the contribution from the last section $c_{3}$ is negligible. The contribution of $c_{1}$ is easily adjusted by multiplying the final solution by $e^{\epsilon x}$ [33]. In our applications, we evaluated the kernel at $N_{k_{x}}=2^{13}$ points, and the value of the offset that guaranteed the wrap-around attenuation by $50 \mathrm{~dB}$ was

$$
\epsilon=\frac{12 k_{x}^{\max }}{2 \pi\left(N_{k_{x}}-1\right) \log e}
$$

which is four times greater than that required for applications of sound reflection 
from the sea bottom [33]. 


\section{Appendix C}

\section{Comparison of the Wide Angle Parabolic Equation Program with the Exact Solution}

In order to validate the accuracy of the three-dimensional wide angle parabolic equation program used in Chapters 6 and 7, we run it for the same scenario as shown in Fig. 5-24 and the result is shown in Fig. C-1. One can clearly see a very good agreement between two results at the wide range (up to $40 \mathrm{deg}$ ) of horizontal angles. This allowed us to reliably use this program for the more complicated environments such as crossing nonlinear internal waves or the large sand waves at the San Francisco Bay. 

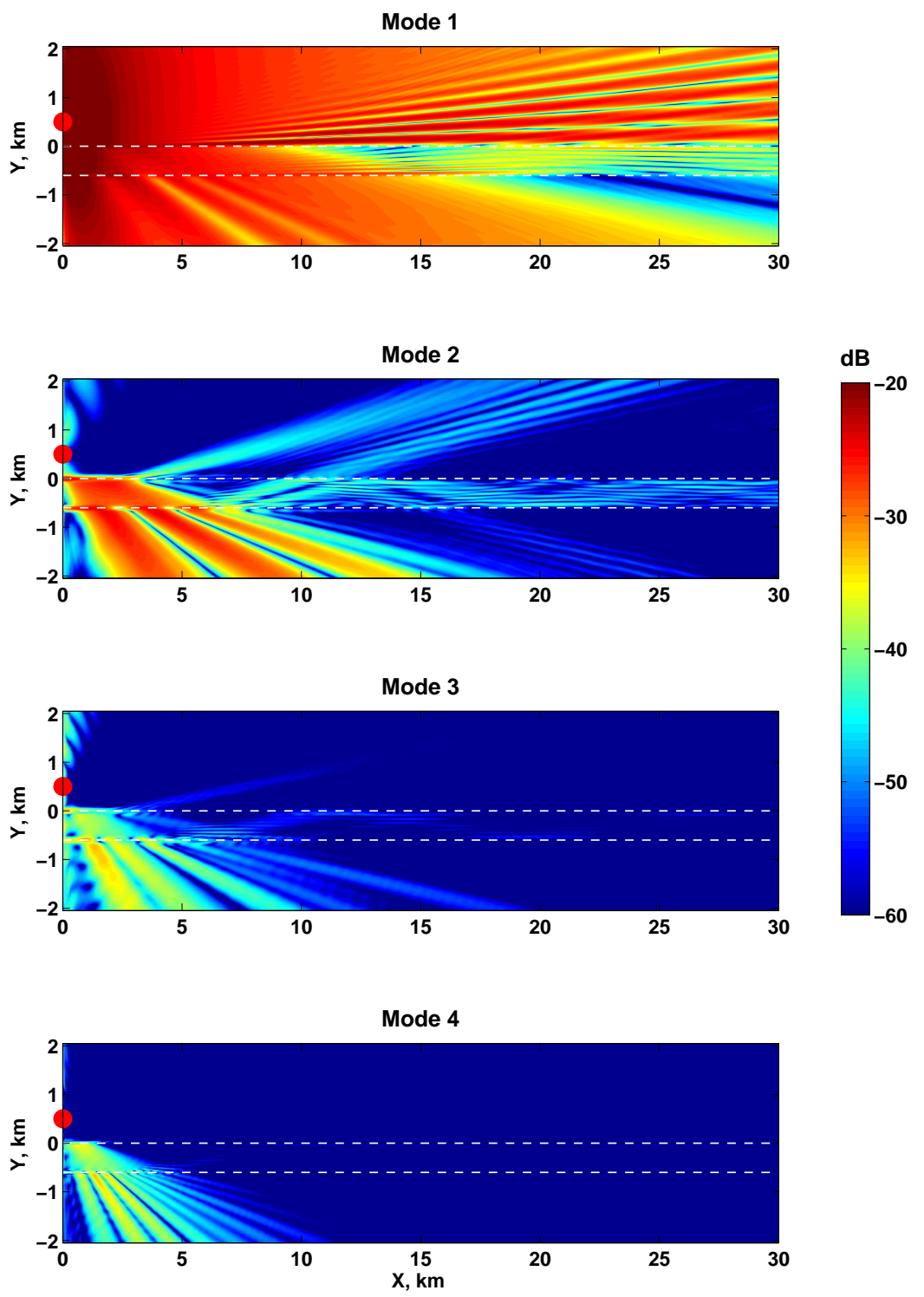

Figure C-1: Output of the three-dimensional wide angle parabolic equation program for the same scenario as shown in Fig. 5-24. 


\section{Bibliography}

[1] L.M. Brekhovskikh, Y.P. Lysanov. Fundamental of Ocean Acoustics, Third Edition, Springer-Verlag, 2002.

[2] C. Garrett, W. Munk. Internal Waves in the Ocean, Ann. Rev. Fluid Mech., Vol. 11, P. 339-369, 1979.

[3] J. Zhou, Z. Zhang, P.H. Rogers. Resonant Interaction of Sound Wave with Internal Solitons in the Coastal Zone, J. Acoust. Soc. Amer., Vol. 90, P. 20422054, 1991.

[4] J.C. Preisig, T.F. Duda. Coupled Acoustic Mode Propagation Through Continental-Shelf Internal Solitary Waves, J. Acoust. Soc. Amer., Vol. 22, P. 256-269, 1997.

[5] J. A. Colosi, S.M. Flatte. Mode Coupling by Internal Waves for MultiMegameter Acoustic Propagation in the Ocean, J. Acoust. Soc. Amer., Vol. 100, P. 3607-3620, 1996.

[6] J.R. Apel, M. Badiey, C.S. Chiu, S. Finette, R. Headrick, J. Kemp, J.F. Lynch, A. Newhall, M.H. Orr, B.H. Pasewark, D.Tielbuerger, A. Turgut, K. Heidt, S. Volf. An Overview of the 1995 SWARM Shallow-Water Internal Wave Acoustic Scattering Experiment, IEEE J. Ocean. Eng., Vol. 22, P. 465-500, 1997.

[7] A. Fredericks, J.A. Colosi, J.F. Lynch, and G. Gawarkiewicz. Analysis of Multipath Scintillations from Long Range Acoustic Transmissions on the New Eng- 
land Continental Slope and Shelf, J. Acoust. Soc. Amer., Vol. 117, P. 1038-1057, 2005.

[8] M. Badiey, J. Lynch, X. Tang, and J.Apel. Azimuthal and Temporal Dependence of Sound Propagation Due to Shallow Water Internal Waves, IEEE J. Ocean. Eng., Vol. 27, P. 117-129, 2002.

[9] B.G. Katsnelson and S.A. Pereselkov. Low-Frequency Horizontal Acoustic Refraction Caused by Internal Wave Solitons in a Shallow Sea, Acoust. Phys., Vol. 46, P. 684-691, 2000.

[10] R. Oba, S. Finette. Acoustic Propagation Through Anisotropic Wave Fields: Transmission Loss, Cross Range Coherence, and Horizontal Refraction, J. Acoust. Soc. Amer., Vol. 111, P. 769-784, 2002.

[11] R. Oba, S. Finette. Horizontal Array Beamforming in an Azimuthally Anisotropic Internal Wave Field, J. Acoust. Soc. Amer., Vol. 114, P. 131-144, 2003.

[12] D.J. Tang, J.N. Moum, J.F. Lynch, P. Abbot, R. Chapmann, P.H. Dahl, T. F. Duda, G. Gawarkiewicz, S. Glenn, J.A. Goff, H. Graber, J. Kemp, A. Maffei, J.D. Nash, A. Newhall. Shallow Water '06, A Joint Acoustic Propagation/Nonlinear Internal Wave Physics experiment, Oceanogr., Vol. 20, P. 156-167, 2007.

[13] A. E. Newhall, T. F. Duda, K. Heydt, J. D. Irish, J. N. Kemp, S. A. Lerner, Sp. P. Liberatore, Y.T. Lin, J. F. Lynch, A.R. Maffei, A.K. Morozov, A. Shmelev, C.J. Cellers, W.W. Witzell. Acoustic and Oceanographic Observations and Configuration Information for the WHOI Moorings from the SW06 Experiment, Woods Hole Oceanogr. Inst., Woods Hole, MA, Tech. Rep. WHOI-200704, 2007.

[14] T.F. Duda, J.M. Collis, Y.T. Lin, A.E. Newhall, J.F. Lynch, H.A. DeFerrari. Horizontal Coherence of Low-Frequency Fixed-Path Sound in a Continental 
Shelf Region with Internal-Wave Activity, J. Acoust. Soc. Amer., Submitted 2011.

[15] M. Badiey, B.G. Katsnelson, Y.-T. Lin, J.F. Lynch. Observation of Acoustic Multipath Arrivals in the Horizontal Plane due to Approaching Nonlinear Internal Waves, J. Acoust. Soc. Amer., Vol. 129, P. EL141-EL147, 2011.

[16] J.F. Lynch, C. Emerson, P.A. Abbot, G.G Gawarkiewitz, A.E. Newhall, Y.T. Lin, T.F. Duda. Are Azimuthal Isotropy and Alongshelf Invariance Found in Low-Frequency Acoustic Propagation Along the New-Jersey Shelfbreak? J. Acoust. Soc. Amer., Submitted 2011.

[17] J.F. Lynch, Y.-T. Lin, T.F. Duda and A.E. Newhall. Acoustic Ducting, Reflection, Refraction, and Dispersion by Curved Nonlinear Internal Waves in Shallow Water, IEEE J. Ocean. Eng., Vol. 35, P. 12-27, 2010.

[18] Y.-T. Lin, T.F. Duda, J.F. Lynch. Acoustic Mode Radiation From the Termination of a Truncated Nonlinear Internal Gravity Wave Duct in a Shallow Ocean Area, J. Acoust. Soc. Amer., Vol. 126, P. 1752-1765, 2009.

[19] Y.-T. Lin, T.F. Duda, J.F. Lynch. Erratum: Acoustic mode radiation from the termination of a truncated nonlinear internal gravity wave duct in a shallow ocean area [J. Acoust. Soc. Am. 126, 1752-1765 (2009)], J. Acoust. Soc. Amer., Vol. 128, P. 947, 2010.

[20] C.J. Jackson. An Atlas of Internal Solitary-like Waves and Their Properties, Second Edition, Global Ocean Associates, 2004.

[21] D.J.P. Swift, G.L. Freeland, R.A. Young. Time and Space Distribution of Megaripples and Associated Bedforms, Middle Atlantic Bight, North American Atlantic Shelf, Sedimentology, Vol. 26, P. 389-406, 1979.

[22] G.V. Middleton, J.B. Southard. Mechanics of Sediment Transport, Soc. Econ. Paleont. Miner., Tulsa. Short Course No. 3., 1997. 
[23] D.B. Reeder, B.B. Ma, Y.J. Yang. Very Large Subaqueous sand Dunes on the Upper Continental Slope in the South China Sea Generated by Episodic, Shoaling Deep-Water Internal Solitary Waves, Marine Geology, Vol. 279, P. 12-18, 2011.

[24] A.N. Serebryany. Monitoring of Non-Linear Internal Waves on Continental Shelf with ADCP: Confirming Known Properties of Non-Linear Waves and Discovering New Effects, Acoustics of Inhomogeneous Media, Ras, Collection of Science Efforts by S. Rybak, Moscow, P.140-146, In Russian, 2004.

[25] M.F. de Koning. The Stochastic Characteristics of Geometric Properties of Sand Waves in the North Sea, M.Sc. Thesis, Univ. of Twente, Netherlands, CEM Research Report 2007R-004/WEM-003, 2007.

[26] W.M. Carey, R.B. Evans. Ocean Ambient Noise. Measurement and Theory, Springer-Verlag, 2011.

[27] K.F. Bowden. Physical Oceanography of Coastal Waters, Ellis Horwood Limited, 1983.

[28] W.H. Munk, G.R. Miller, F.E. Snodgrass, N.F. Barber. Directional Recording of Swell from Distant Storms, Phil. Trans. R. Soc. Lond., Vol. 255, P. 505-584, 1963.

[29] Y. Goda. Analysis of Wave Grouping and Spectra of Long-Travelled Swell, Rep. of the Port and Harbour Res. Inst. Ministry of Transport, Japan, Vol. 22, 1983.

[30] E.L. Shroyer, J.N. Moum, J.D. Nash. Nonlinear Internal Waves over New Jersey's Continental Shelf, J. Geophys. Res., Vol. 116, 16 PP, 2011.

[31] D.H. Johnson, D.E. Dudgeon. Array Signal Processing. Concepts and Techniques, Prentice-Hall, Inc, 1993.

[32] M.B. Porter. The KRAKEN Normal Mode Program, SACLANTTECN Memorandum No. SM-245, SACLANT Undersea Research Centre, La Spezia, Italy, 1991. 
[33] F.B. Jensen, W.A. Kuperman, M.B. Porter, H. Schmidt. Computational Ocean Acoustics, Springer-Verlag, 1993.

[34] G.V. Frisk. Ocean and Seabed Acousics, Prentice-Hall, Inc, 1994.

[35] A.D. Pierce. Extension of the Method of Normal Modes to Sound Propagation in an Almost Stratified Medium, J. Acoust. Soc. Amer., Vol. 37, P. 19-27, 1965.

[36] H. Weinberg, R. Burridge. Horizontal Ray Theory for Ocean Acoustics, J. Acoust. Soc. Amer., Vol. 55, P. 63-79, 1974.

[37] C.T. Tindle, L.M. O'Driscoll, C.J. Higham. Coupled Mode Perturbation Theory of Range Dependence, J. Acoust. Soc. Amer., Vol. 108, P. 76-83, 2000.

[38] T.F. Duda, J.C. Preisig. A Modeling Study of Acoustic Propagation Through Moving Shallow-Water Solitary Wave Packets, IEEE J. Ocean. Eng., Vol. 24, P. 16-32, 1999.

[39] M. Badiey, B.G. Katsnelson. Frequency Dependence and Intensity Fluctuations due to Shallow Water Internal Waves, J. Acoust. Soc. Amer., Vol. 122, P. 747$760,2007$.

[40] R.H. Headrick. Analysis of Internal Wave Induced Mode Coupling Effects on the 1995 SWARM Experiment Acoustic Transmissions, Ph.D. Thesis, MIT/WHOI Joint Program, MIT/WHOI 97-21, 1997.

[41] K.G. McMahon, L.K. Reilly-Raska, J.F. Lynch, T.F. Duda, W.L. Siegmann. Horizontal Lloyd Mirror Patterns From Straight and Curved Nonlinear Internal Waves, J. Acoust. Soc. Amer., in press.

[42] C.J. Higham, C.T. Tindle. Coupled Perturbed Modes and Internal Solitary Waves, J. Acoust. Soc. Amer., Vol. 113, P. 2515-2522, 2003.

[43] J.F. Lynch, J.A. Colosi, G.G. Gawarkiewicz, T.F. Duda, A.D. Pierce, M. Badiey, B. G. Katsnelson, J.E. Miller, W. Siegmann, C-S. Chiu, A. Newhall. Consideration of Fine-Scale Coastal Oceanography and 3-D Acoustic Effects 
for the ESME Sound Exposure Model, IEEE J. Ocean. Eng., Vol. 31, P. 33-48, 2006.

[44] P.G. Frank, P.G Bergmann, A.Yaspan. Ray Acoustics. In "Physics of Sound in the Sea", Peninsula Publishing, 1981.

[45] W.M. Carey. Lloyd's Mirror-Image Interference Effects, Acoust. Today, Vol. 3, P. 14-20, 2009.

[46] B.G. Katsnelson, V. Grigorev, M. Badiey, J.F. Lynch. Horizontal Interference Structure of the Sound Field in the Presence of Moving Internal Waves and Estimation of Angle of Horizontal refraction, J. Acoust. Soc. Amer., Vol. 128, P. 2334, 2010.

[47] E. Merzbacher. Quantum Mechanics, Second edition, John Wiley Sons, Inc., 1970.

[48] P.M. Morse, H. Fechbach. Methods of Theoretical Physics, McGraw-Hill, 1953.

[49] F.B. Jensen, H. Schmidt. Subcritical Penetration of Narrow Gaussian Beams into Sediments, J. Acoust. Soc. Amer., Vol. 82, P. 574-579, 1987.

[50] M. Badiey, B. Katsnelson, J.F. Lynch, S. Pereselkov, and W. Siegmann. Measurement and Modeling of 3-D Sound Intensity Variations due to Shallow Water Internal Waves, J. Acoust. Soc. Amer., Vol. 117, P. 613-625, 2005.

[51] K.B. Smith. Convergence, Stability, and Variability of Shallow Water Acoustic Predictions Using a Split-Step Fourier Parabolic Equation Model, J. Comp. Acoust., Vol. 9, P. 243-285, 2001.

[52] R. Cheng, P.J. Morris. K.S. Brentner. A Three Dimensional Parabolic Equation Method for Sound Propagation in Moving Inhomogeneous Media, J. Acoust. Soc. Amer., Vol. 126, P. 1700-1710, 2009. 
[53] S.R. Rutherford. An Examination of Mode Coupled Theory as Applied to Underwater Sound Propagation, Applied Research Laboratories, Austin, TX, Tech. Rep. ARL-TR-79-44, 1979.

[54] J.A. Fawcett, T.W. Dawson. Fourier Synthesis of Three-Dimensional Scattering in a Two-Dimensional Oceanic Waveguide Using Boundary Integral Equation Methods, J. Acoust. Soc. Amer., Vol. 88, P. 1913-1920, 1990.

[55] R.B. Evans. A Coupled Mode Solution for Acoustic Propagation in a Waveguide With Stepwise Depth Variations of a Penetrable Bottom, J. Acoust. Soc. Amer., Vol. 74, P. 188-195, 1983.

[56] M.B. Vinogradova, O.V. Rudenko, A.P. Sukhorukov. Wave Theory (Teoriya Voln). In Russian, Nauka, 1979.

[57] D.C. Twitchell. Bedform Distribution and Inferred Sand Transport on Georges Bank, Unites States Atlantic Continental Shelf, Sedimentology, Vol. 30, P. 695$710,1983$.

[58] I. Dyer. Statistics of Sound Propagation in the Ocean, J. Acoust. Soc. Amer., Vol. 48, P. 337-345, 1970.

[59] F. Dyson, W. Munk, and B. Zelter. Interpretation of Multipath Scintillations Eleuthera to Bermuda in Terms of Internal Waves and Tides, J. Acoust. Soc. Amer., Vol. 59, P. 1121-1133, 1976.

[60] T. F. Duda. Initial Results from a Cartesian Three-Dimensional Parabolic Equation Acoustical Propagation Code, Woods Hole Oceanogr. Inst., Woods Hole, MA, Tech. Rep. WHOI-2006041, 2006.

[61] R.H. Hardin, F. D. Tappert, Applications of the Split-Step Fourier Method to the Numerical Solution of Nonlinear and Variable Coefficient Wave Equations, SIAM Rev., Vol. 15, P. 423423, 1973. 
[62] D.J. Thomson, N.R. Chapman. A Wide-Angle Split-Step Algorithm for the Parabolic Equation, J. Acoust. Soc. Amer., Vol. 74, P. 18481854, 1983.

[63] A.R. Smith, K.B. Smith. Mode Functions for the Wide-Angle Approximation to the Parabolic Equation, J. Acoust. Soc. Amer., Vol. 103, P. 814-821, 1998.

[64] J. Pedlosky. Waves in the Ocean and Atmosphere, Springer-Verlag, 2003.

[65] K.C. Ewans. Directional Spreading in Ocean Swell, Proc. Int. Symp. WAVES 2001, P. 517-529, 2002.

[66] Y. Goda. Random Seas and Design of Maritime Structures, Third Edition, World-Scientific, Advanced Series on Ocean Engineering, Vol. 33, 2010.

[67] H. Mitsuyasu, F. Tasai, T. Suhara, S. Mizuno, M. Ohkusu, T. Honda, K. Rikiishi. Observations of the Directional Spectrum of Ocean Waves Using a Cloverleaf Buoy, J. Phys. Oceanogr., Vol. 5, P. 750-760, 1975.

[68] Y.Goda, Y. Suzuki. Computation of Refraction and Diffraction of Sea Waves with Mitsuyasu's Directional Spectrum, Tech. Note of Port and Harbour Res. Inst., Vol. 230, 1975.

[69] M.D. Collins. A split-step Pade solution for the parabolic equation method, J. Acoust. Soc. Amer., Vol. 93, P. 17361742, 1993.

[70] A.K. Morozov, J.A. Colosi. Entropy and Scintillation Analysis of Acoustic Beam Propagation Through Ocean Internal Waves, J. Acoust. Soc. Amer., Vol. 117, P. 1161-1623, 2004.

[71] P.L. Barnard, D.M. Hanes, D.M. Rubin. Giant Sand Waves at the Mouth of San Francisco Bay, EOS Transactions, Vol. 87, P. 285-289, 2006.

[72] Seafloor Mapping Lab Data Library: http://seafloor.csumb.edu/SFMwebDATA.htm

[73] K.G. McMahon, J.F. Lynch, Y.T. Lin, N. Xiang, W.L. Siegmann. Nonlinear Internal Wave Parameter Influences on Energy Propagation Using Radiative Transport Theory, J. Acoust. Soc. Amer., Vol. 129, P. 2458-2458, 2011. 
[74] P.H. Milne. Underwater Acoustic Positioning Systems, Spon Press, 1983.

[75] M.B. Porter, H.P. Bucker. Gaussian beam tracing for computing ocean acoustic fields, J. Acoust. Soc. Amer., Vol. 82, P. 1349-1359, 1987.

[76] G.J. Orris, M.D. Collins. The Spectral Parabolic Equation and ThreeDimensional Backscattering, J. Acoust. Soc. Amer., Vol. 96, P. 1725-1731, 1994.

[77] H. Schmidt. "SAFARI": Seismo-Acoustic Fast Field Algorithm for Range Independent Environments. User's Guide. Rep. SR-113, SACLANT Undersea Research Centre, La Spezia, Italy, 1988. 